UC-NRLF

|||||||||||||||||||||||||||||||||||| |||||

||||||||||||||||||||||||||||||||||||||||||||||||

B 4 091 960 


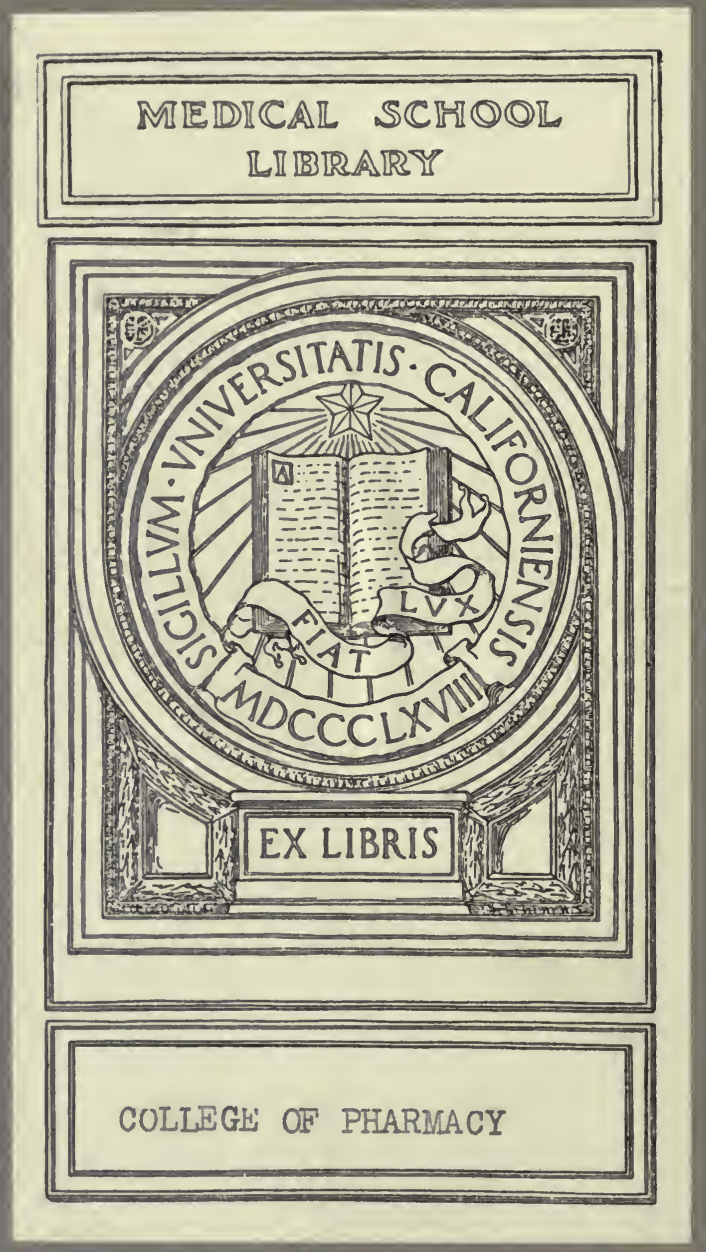





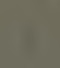

$+$

(3) $+1+1$ 


$$
\text { . }
$$
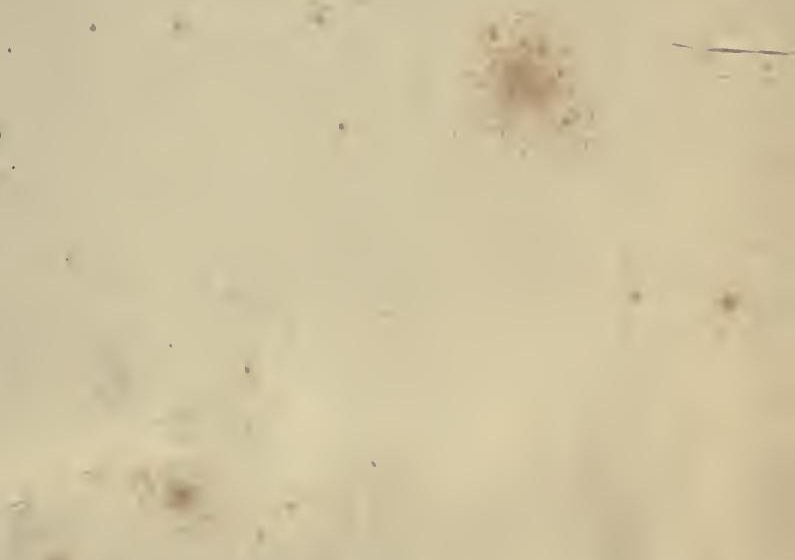

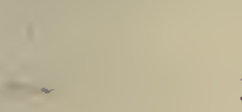




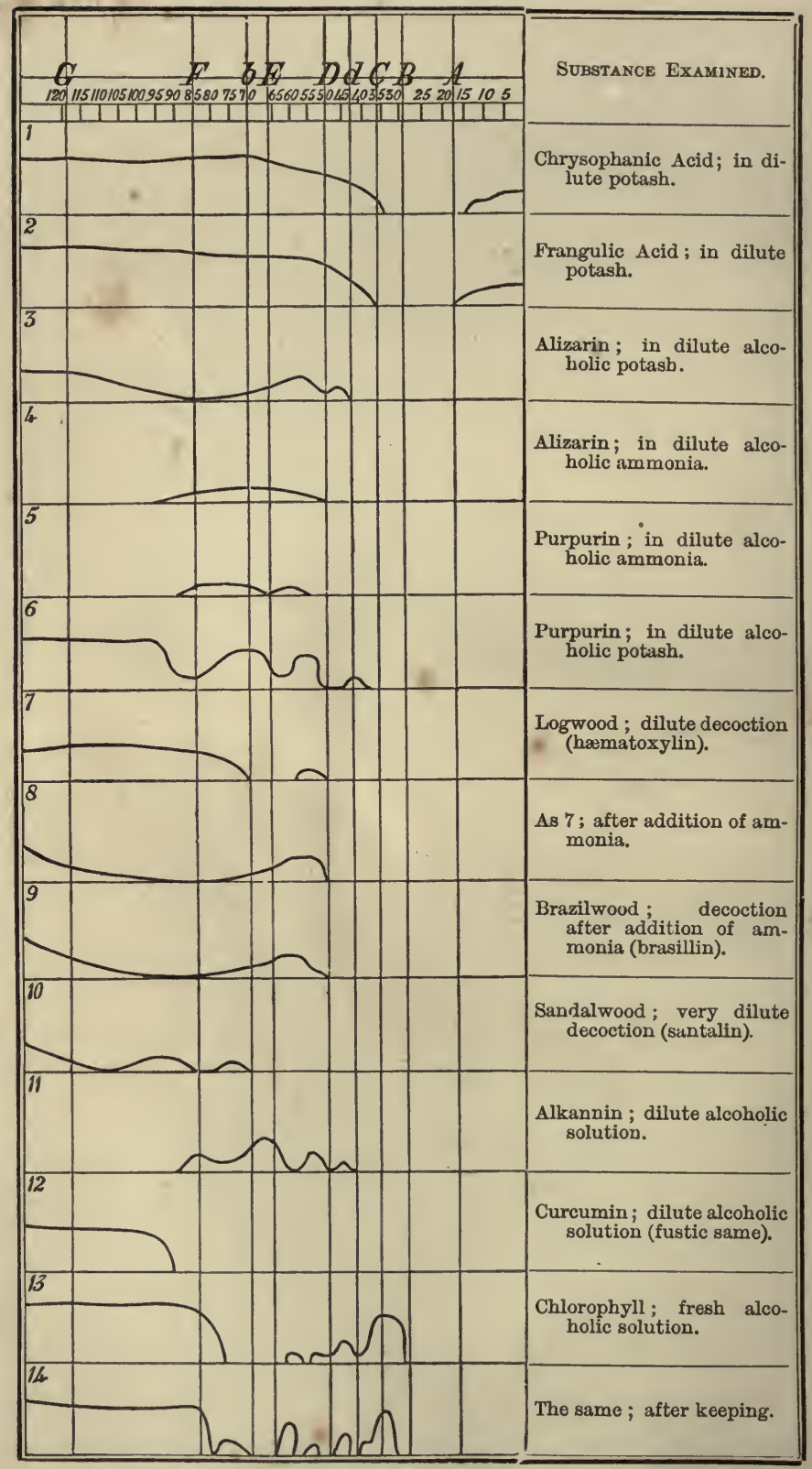


W. T. WENLELL,

Wan Francisco, Cal.

PLANT ANALYSIS:

QUALITATIVE AND QUANTITATIVE.

BY

G. DRAGENDORFF, Рн.D.,

PROFESSOR OF PHARMACY IN THE UNIVERSITY OF DORPAT, RUSSIA.

Translated from the German

BY

HENRY G. GREENISH, F.I.C.

\section{Oalisonia College of Pharmacy}

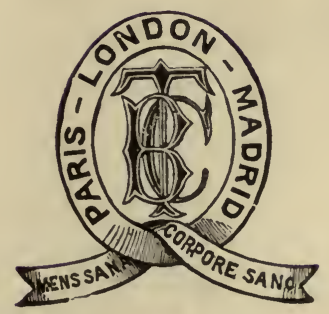

\section{LONDON :}

B A I L L I ÈR E, TIN D A L L, A N D C OX, 20, KING WILLIAM STREET, STRAND.

1884.

[All Rights Reserved.]

R 
Digitized by the Internet Archive in 2007 with funding from Microsoft Corporation 


\section{TRANSLATOR'S PREFACE.}

Soon after the publication in German of Professor Dragendorff's 'Pflanzenanalyse,' it was suggested to me that an English translation of the work would supply a want keenly felt by both English chemists and English pharmacists.

A thorough knowledge of the German language and a practical acquaintance with many of the processes described, gained whilst a pupil in the author's laboratory, would, it was thought, enable me to offer a translation of trustworthy accuracy; and this has been my endeavour. Such alterations or additions as have been considered needful have been made in the text, the proof-sheets of which have been submitted to the author.

Most of the references have been checked, as accuracy in this particular was deemed very important. To many of them, however, access could not easily be had; but it is hoped that even in these cases very few will be found to be incorrect. To secure to English readers the usefulness of the numerous quotations, reference has been frequently made, in brackets, to abstracts or translations that have appeared in English journals.

One word has been employed in a somewhat unusual sense. The solution obtained by treating a substance with spirit is called a 'tincture,' with cold water an 'infusion,' and so on. All such solutions have been included in the general term 'extract;' the latter will not, therefore, necessarily mean the dry residue commonly called 'extract.'

The name 'petroleum spirit' sufficiently indicates the origin of 
the liquid. A petroleum spirit boiling above $60^{\circ} \mathrm{C}$. should not be used. Benzene should boil at $80-81^{\circ} \mathrm{C}$. ('Die gerichtlichchemische Ermittelung von Giften,' Dragendorff, 1876.)

The index will be found more copious than in the original; it has been compiled from the English text.

The high reputation of the author and the favourable reception accorded to his 'Pflanzenanalyse' are a sufficient guarantee for the value of the work.

THE TRANSLATOR.

LoxDos, October 1st, 1883. 


\section{AUTHOR'S PREFACE.}

WHILST engaged in collecting the material for my 'Ermittelung von Giften,' I formed the intention of utilizing the knowledge then acquired of the alkaloidal and other constituents of plants to improve and extend the present methods of plant analysis. In accordance with this intention I subsequently discussed in my 'Chemische Werthbestimmung' the detection and estimation of the active principles of some powerful drugs, and at the same time promised further communications on allied substances.

In the meantime, I gradually became convinced of the need of devising a process of analysis that should include as many as possible of the more important constituents of plants. Such a process was, I thought, a desideratum, as I had frequently observed that the methods of examination published in some of my researches were adopted by other chemists in cases in which I myself should have deviated from them.

This consideration was mainly instrumental in inducing me to carry my plan into execution more rapidly than was originally contemplated. No one can be more thoroughly aware than I am myself of the insufficiency of the material at present available for the construction of a systematic process of analysis, nor can anyone be more conscious of the necessity for sifting and improving the contents of the following chapters. I may, however, be permitted to remark that in proposing to my pupils subjects for scientific investigation, I have never lost sight of the plan I had formed, and I have been able to benefit by the results of upwarls 
of one hundred dissertations or communications published by myself or by my scholars.

Comparatively few chemists will have learnt, as I have done, that nothing can tend so much to the end aimed at as increased activity in this much-neglected branch of chemistry; and it was the hope of stimulating young chemists to steady, persevering work in testing the methods now placed before them, and devising better ones, that finally decided me. I doubt the possibility of making, without assistance, such progress as I think necessary; and I trust, therefore, that the publication of this little work will be followed by an increase in the number of my fellow-workers.

As will be explained in the introduction, I have endeavoured to construct a method that shall comprise at once both the qualitative and the quantitative, micro- as well as macro-chemical analysis of plants and their constituents. All widely distributed vegetable substances are to be included, the detection of rarer ones facilitated, and the method so arranged that other principles not hitherto observed shall, if present, attract the attention of the investigator.

An exhaustive treatise on all the known constituents of plants would naturally have obscured the method of examination. This result I have endeavoured to avoid by compressing the method of examination proper (Part I.) into the smallest possible limits ; and by following it up with further observations (Part II.) on the characters, etc., of the substances there mentioned. Numerous notes and a systematic, as well as alphabetical, index will guard the reader from confusion.

I have been compelled to restrict myself to the treatment of the more important constituents of plants, that is, those that are of importance to the plant itself, or that play an important part in its economical application. The extracts in which rarer or less important substances are to be looked for have been pointed out, but it has been left for the reader himself to gain further information about them from other sources. Numerous references will aid him in his search, and also direct his attention to a number of analyses that may be of service to him in modifying or extending the process here recommended. 
I have assumed in my readers an acquaintance with the leading principles of general and analytical chemistry, and have, therefore, passed over parts of the latter, such as ultimate and ashanalysis, since these have been fully treated of elsewhere. Subjects that have been discussed at length in my 'Ermittelung von Giften,' and 'Chemische Werthbestimmung starkwirkender Droguen,' have been referred to as briefly as possible. An ultimate analysis is, of course, frequently necessary in order to demonstrate the identity of a substance isolated during the investigation with some other known body. I have, therefore, collected analyses of the constituents of plants, and have arranged them both alphabetically and according to the percentage of carbon they contain.

THE AUTHOR. 



\section{SYSTEMATIC INDEX.}

$\S 1$. General Remarks, p. 1.-\$2. Object of the Work; Division of Matter, p. 2.-\$3. Leading Principles in the Analysis of Plants, p. 3

METHOD OF EXAMINATION FOR THE MORE IMPORTANT CONSTITUENTS OF PLANTS

I. Prelininary Operations. Estimation of Moisture and Ash .

$\S 4$. Drying the Materials, p. 5.-§5. Treatment of Fresh Plants, p. 6. - $\$$. Pulverization, p. 6.-§7. Estimation of Ash, p. 7

II. Examination of the Substances Soluble in Petroleum Spirit ; Ethereal and Fixed Oils, Wax, etc. . . . .

§ 8. Value of Petroleum Spirit in the Analysis of Plants, p. 8.$\S 9$. Methods of Extraction, p. 8. - $\$ 10$. Treatment of Fresh Aromatic Vegetable Substances, p. 10

Examination of the Fixed Oil

-

$\S$ 11. Macroscopical and Microscopical Detection; Total Estimation, p. 10.-\$ 12. Composition, Qualitative; Oleic and Linoleic Acids, p. 11.—§. 13. Quantitative ; Estimation of Glycerine, p. 12.-\$14. Cetyl-, Cerotyl-, Melyl-Alcohol, p. 13.$\S 15$. Volatile Fat-Acids, p. 13.-§ 16. Non-volatile FatAcids; Separation, p. 14. $-\$ 17$. Determination of Melting. Point, p. 14. $-\$ 18$. Melting-Points of the more important FatAcids and Mixtures of the same, p. 15.-\$19. Further Remarks on Oleic, Ricinoleic Acid, etc., p. 18.

Chlorophyll and Alkaloids Extracted Simultaneously with The Frxed OrL .

$\S 20$. Optical Properties and Detection of Chlorophyll, p. 19.$\S 21$. Influence of Fixed Oil in Determining the Solution of Alkaloids, p. 20.

Examination of the Ethereal Oil

$\S 22$. Detection and Estimation, p. 21.-§ 23. Estimation in the Presence of Fixed Oil and Resin, p. 22.-\$24. Distillation of Larger Quantities of Ethereal Oil, p. 23.-\$ 25. Examination of the Aqueous Distillate for Volatile Acids, Formic, Acetic, Acrylic, Toxicodendric, Salicylous Acid, p. 23.—\$26. Salicylic, Benzoic, Cinnamic Acid, Styracin, Cinnameïn and Aldehydes of above Acids, p. 24.- $\S 27$. Physical Properties of Ethereal Oils ; Umbelliferone, p. 25.—§ 28. Reactions, p. 26.-§ 29. Ethereal Oils containing Nitrogen and Sulphur, p. 26. - $\$ 30$. Constituents of Ethereal Oils, p. 27.-\$31. Hydrocarbons and Oxygenated Constituents, Stearoptenes, p. 28.- $\$$ 32. Other 
Constituents, p. 28.—§ 33. Aldehydes, p. 29.—§ 34. Volatile Acids, p. 29. - $\$ 35$. Ethereal Salts and the Alcohols contained in them ; Primary, Secondary, and Tertiary Alcohols, p. 29.

III. Examination of the Substances Soluble in Ether : Resins AND THEIR Allies

§ 36. Methods of Extraction ; Fixed Oil, p. 31.—§ 37. Chlorophyll, p. 32. - $\$ 38$. Portion of the Ethereal Extract Soluble in Water; Hæmatoxylin, Gallic Acid, Glucosides, Alkaloids, etc., p. 32.$\S 39$. Portion Soluble in Alcohol, p. 33. - $\$ 40$. Microchemical Examination; Treatment of the Substances dissolved by Ether with various Solvents; Crystallization, etc., p. 33. - $\$ 41$. Behaviour of Resin to Aqueous and Alcoholic Potash, Sulphuric Acid, Nitric Acid, Bromine, etc., p. 34.-\$ 42. Action of fused Potash, Resorcin, Phloroglucin, Pyrogallol, Protocatechuic and Paroxybenzoic Acids, p. 34.- $\$ 43$. Dry Distillation of Resin; Umbelliferone, Pyrocatechin, p. 36.-\$ 44. Examination of that Part of the Ethereal Extract dissolved by Alcohol; Pæoniofluorescin, Chrysophanic Acid, etc., p. 36.-\$ 45. Acids produced by the Action of Alkalies on Anhydrides ; Santonin, etc., p. 36. - $\$ 46$. Direct Extraction with Ether, p. 36 .

IV. Examination of the Substances Soluble in Absolute Alcohol ; Resins, Tannins, Bitter Principles, Alkaloids, Glucoses, etc.

§ 47. Methods of Extraction; Estimation of Total Substances dissolved, p. 38. - $\$ 48$. Estimation of the Portion Soluble in Water; Phlobaphenes, Alkaloids, etc., p. 38.

Examination of Tannin

$\S 49$. Detection, p. 39. $§ 50$. Detection continued, p. $3 \dot{9} .-\S 5 \dot{1}$. Reactions of most Tannins; Microchemical Detection; Alcohol more suitable for their Extraction than Water, p. 40. - $\$ 52$. Methods for their Estimation : I. Acetate of Lead, p. 41; II. Acetate of Copper, p. 42 ; III. Stannous Chloride, p. 42 ; IV. Tartar Emetic, p. 42; V. Acetate of Zinc, p. 43 ; VI. Ferric Acetate, p. 43 ; VII. Permanganate of Potassium, p. 43 ; VIII. Chlorinated Lime, Iodic Acid, Iodine, p. 45 ; IX. Caustic Potash and Atmospheric Air, p. 45 ; X. Cinchonine, p. 45 ; XI. Hide, p. 46; XII. Gelatine, p. 46. - 53. Tannic and Gallic Acid, p. 47

Examination for Glucosines, Alkaloids, etc.

$\S 54$. By the Method of Agitation, p. 48.-\$55. List of Bitter Principles, Acids, etc, removable from Acid Solution by Agitation with Petroleun Spirit, Benzene, Chloroform, p. 49.-\$56. Extraction of Alkaloids from Ammoniacal Solution, p. 49. - $\$ 57$. Direct Test for Glucosides, Alkaloids, etc., p. 50.-§ 58. Isolation and Purification of Substances not removable by Agitation; Separation from Glucose, etc., p. 51.-\$59. Separation of certain Glucosides and Bitter Principles from Tannin, etc., p. 52. - $\$ 60$. Decomposition of Compounds of Lead with Bitter Principles, etc., p. 52.- $\S 61$. Detection of the Glucosidal Nature of a Substance, p. 53.- $\$ 62$. Other Reactions of Glucosides, p. 54. - $\$ 63$. Alkaloids not Isolated by the Method of Agitation ; Group-reagents ; Lassaigne's Nitrogen Test, p. 55. - $\$$ 64. Isolation by Precipitation with Potassio-mercuric Iodide, etc., p. 57.-§65. Estimation, p. 58.-\$66. Estimation 
of Theine, p. 62.- $\$ 67$. Estimation of Total Alkaloids in Cinchona, p. 62.-§ 68. Acidimetric Estimation, p. 63.-§69. Separation of Alkaloids from one another, p. 63. $-\$ 70$. Glucoses Soluble in Alcohol, p. 64.

V. Examination of Substances Soluble in Water: Mucilage, Saponin, Acids, Glucoses, Saccharoses, etc.

$\S 7$. Method of Extraction, p. 65.-\$ 72. Estimation of Total Substances Dissolved, p. 65.

Examination for Vegetable Mucilage, Dextrin, Levelix, Triticin ; Sinistrin . $\quad$. . . . .

$\S 73$. Detection and Estimation of Mncilage, p. 65.-\$ 74. Vegetable Albumin and Tarsoates Present in Mucilage-precipitate, p. 66. - $\$ 75$. Inulin, p. 66. - $\$ 76$. Dextrin, Levulin, Sinistrin, Triticin; Estimation, p. 67.

Saponin and its Allies . . . . .

$\S$ 77. Separation from Dextrin, etc., p. $67 .-\$ 78$. Estimation, p. $68 .-\$ 79$. Digitonin, p. 69.

Examination for AcIDs

$\S 80$. Precipitation with Acetate of Lead, p. 69.-\$ 81. Malic, Fumaric, Oxalic, Racemic, Citric, Aconitic, Tartaric Acid; Marattin, p. 70. $\$$ 82. Volumetric Estimation of the foregoing Acids. Free and Combined Acid. Mineral Acids, p. 71.

Examination for Glucoses, Saccharoses, Etc. . . .

§ 83. Volumetric Estimation of Glucose with Fehling's Solution ; Gravimetric Estimation with Copper, p. 72.-\$ 84. Knapp's Method; Sachsse's Method, etc., p. 73.-\$ 85. Influence of Saccharoses, p. 75. - $\$ 86$. Estimation of Saccharose in presence of Glucose, p. 75.-\$ 87. Estimation of Saccharose alone; Inversion, p. 75.-\$88. Distinguishing Tests for Saccharose and Glucose, p. 76. - $\$$ 89. Distinctive Characteristics of the Various Saccharoses and Glucoses; Purification, p. 76. $\$$ 90. Soluble Modification of Arabic Acid; Albuminous Substances not precipitated by Alcohol, p. 76.-\$91. Mannite and its Allies, p. 77.

Examination for Albuminoids Soluble in Water, Ammonia, Amides, Nitric Acid .

§ 92. Detection and Estimation; Microchemical Detection ; Protoplasm, Cell-nucleus, Crystalloids, p. 78.— $\$ 93$. Estimation of Legumin, Globulin, and Allied Substances, p. 79.—§94. Vegetable Albumin, p. 79.- $\$ 95$. Estimation of Total Albuminoids Soluble in Water; (a) By Precipitation with Tannin, p. 80.$\S$ 96. (b) From the Nitrogen, p. 80.- $\$ 97$. Estimation of Am. monia, p. 81.—\$98. Amido-compounds, p. 82.—\$99. Estimation of Nitric Acid (a) by Schulze's Method, p. 83.- $\S 100$. (b) By Wulfert-Schloessing's Method, p. 85.-§ 101. Sclerotic and Cathartic Acid, etc., p. 86.

Examination For INULiN

$\S$ 102. Characteristic Properties of Inulin and Inuloid, p. 86.

VI. Examination of the Substances Soluble in Dilute Soda: Metarabic Acid, Albuminoids, Phlobaphene, etc.

$\S 103$. Method of Extraction, p. 88. - $\$ 104$. Detection and Estimation of Albumen, p. 88. - $\$ 105$. Estimation, p. 88.-\$106. Nitrogenous Substances not dissolved by Dilute Soda, p. 89. - 
$\S 107$. Mucilaginous and Albuminous Substances, Phlobaphene, etc., not Precipitated by Acids, p. 89. $-\S 108$. Phlobaphene, Polyporic Acid, Humus, etc., p. 90.

VII. Examination of Substances Soluble in Dilute Hydrochloric Acid ; Starch, Pararabin, Oxalate of Calcium, etc. . $\S 109$. Extraction, p. 91. - $\$ 110$. Estimation of Oxalate of Calcium, p. 91.- $\$ 111$. Estimation of Oxalate of Calcium and Pararabin, p. 92.— $\$ 112$. Estimation of Pararabin, p. 93.— $\$ 113$. Estimation of Oxalate of Calcium and Starch, p. 93.- $\$ 114$. Estimation of Oxalate of Calcium, Starch, and Pararabin, p. 93.$\S 115$. Estimation of Starch, p. 93.

ViII. Estimation of Lignin and irs Allies, and of Cellulose . § 116. Lignin, Incrusting and Cuticular Substances, Suberin, p. 95. - $\$ 117$. Estimation of Cellulose, p. 96.

Concluding Remarks

$\S$ 118. Remarks on the Method of Analysis recommended, p. 97. - $\$ 119$. On the Object of Plant Analysis, p. 97.

SPECIAL METHODS; SUPPLEMENTARY NOTES, ETC.

Fats and their Constituents, Cholesterin, Filicin, etc.

PAGE

$\S 120$. Estimation of Fat in General ; Apparatus for Extraction, p. 99.-\$121. Resinification, p. 101.-\$122. Elaidin-test, p. 101. -\$123. Behaviour to Sulphuric Acid, p. 102.-\$124. Behaviour to other Reagents, p. 102.- $\$ 125$. Detection and Estimation of Free Fat Acids contaminating Fixed Oils, p. 105.- $\$ 126$. Detection and Estimation of Cholesterin, Phytosterin, Filicin, Kosin, Euphorbon, Lactucon, Lactucerin, Echicerin, Cynanchocerin, Helenin, Coumarin, Melilotic Acid, Styrol, Myroxocarpin, Diosmin, Kämpferid, Asaron, Angelicin, Anemonol, Capsicin, Capsaicin, Amyrin, Bryoïdin, p. 106.$\S 127$. Caoutchouc, p. 109.—§ 128. Estimation of Glycerine, p. 109.-§ 129. Cetyl-, Cerotyl-, Melyl-alcohol; Cerotene; Vegetable Wax; Microchemical Detection of Wax, p. 110. $\S 130$. Estimation of Oleic Acid, Linoleic Acid, Lauric Acid; Separation of the latter from Oleic and Myristic Acid; of Oleic from Stearic Acid, p. 111.-§ $\$ 131$. Separation of Fatacids from Resin-acids, p. 112.

Chlorophyll and its Alties

$\S 132$. Remarks on the Chemistry of Chlorophyll, p. 113.$\S 133$. Possibility of Estimating: p. 115.-§ 134. Erythrophyll and Chlorophyllan, etc., p. 115.-\$135. Xanthophyll, Hypochlorin, Etiolin, Anthoxanthin, p. 116.

Ethereal Oils, Volatile Acids, etc.

$\S$ 136. Examples of Estimation, p. 117.- $\$ 137$. Estimation with Bisulphide of Carbon, p. 118. $-\S 138$. Mixtures of Fixed and Ethereal Oils, Resin, etc., p. 118.—§ 139. Volatile Acids : Angelic, Methylcrotonic, Capric, Caprylic, Enanthic, Caproic, Valerianic, Butyric, Propionic, Acetic, Formic Acids and their Separation, p. 119.-\$140. Identification of Volatile Acids by Saturating Power, etc., p. 120.-\$141. Optical Tests for Ethereal Oils, Solubility, p. 120. $-\$ 142$. Colour-reactions of Ethereal Oils, p. 121.— $\$ 143$. Fractional Distillation, p. 124. - § 144. Examples of Analyses, p. 125. 
Resins, Anthraquinone-Derivatives, Gallic Acid, Bitter PrinCIPLES, ETC.

$\$ 145$. Coniferous Resin-Acids; Podocarpic Acid, Phyllic Acid, Mongumic Acid, Pæonia acid, Chrysin, etc.; More important Methods of Isolating Resin-Acids, p. 127.- $\$ 146$. More important Commercial Resins; Estimation of Ethereal Oil, Mucilage, etc., p. 129.-\$147. Pæonio-fluorescin, p. 131.$\S$ 148. Anthraquinone-derivatives, Chrysophanic Acid, Chrysarobin, Emodin, Frangulic Acid, Alizarin, Purpurin, Sclererythrin, Ruberythric Acid, Rhinacanthin, Alkannin, Bixin, Curcumin, etc., p. 131. - $\$ 149$. Recognition of Anthraquinonederivatives, p. 136. - $\$ 150$. Hæmatoxylin, Brasillin, Santalin, p. 136. - $\$ 151$. Gallic Acid, Catechin, Pyrocatechin; Detection, Estimation, etc., p. 137.-\$152. Quercitrin, Quercetin, Thujin, Rutin, Robinin, Luteolin, Gentisin, Constituents of Podophyllin, p. 138. - $\$ 153$. Jalapin and Allied Resin-glucosides; Convolvulin, Tampicin, Turpethin, etc., p. 140.-\$154. Santonin ; Estimation, p. 141. - $\$ 155$. Picrotoxin, Digitalin, Digitoxin, Digitaleïn, Digitonin, Digitin, Coriamyrtin, Ericolin, Vanillin (Estimation), Ostruthiin, Peucedanin, Oreoselon, Athamanthin, Laserpitin, Cubebin, Betulin, Anacardic Acid, Cardol, p. 142. $\S 156$. Other Bitter Principles Soluble in Ether; Absinthiin, Elaterin, Hop-Bitters, Meconin, Meconic Acid, Methysticin, Quassiin, etc., p. 146. - § 157. Lichen Acids and their Allies: Roccellic, Lecanoric, Orsellinic, Gyrophoric, Parellic, Patellaric, Evernic, Everninic, Usnic, Carbusnic, Vulpic, Frythric, Beta-erythric, Cetraric, Lichenostearic, Stictic, Lobaric, Atranoric Acid; Ceratophyllin, Picroerythrin, Picrolichenin, Variolinin, Zeorin, Sordidin, Calycin, etc., p. 149.-§158. Orcin and Betaorcin; Estimation of Orcin, p. 152.

Taxiss

$\S 159$. Constitution, p. 152. - $\$ 160$. Glucosidal Nature or otherwise Decomposition-products, Phlobaphene, etc., p. 153.-§ 161. Proneness to Decomposition, p. 154. $\$$ 162. Preparation in a State of Purity, p. 155. - $\$ 163$. Tannic Acids sparingly Soluble in Water: Tannins of Alder and Hops, p. 156.- $\$ 164$. Occurrence of two different Tannins in the same Plant, p. 156.$\S 165$. Notes on the more important Tannins; Tannic Acids from Catechu, Rhatany, Kino, Tormentilla, Bistort, Horsechestnut, Sumach, Myrobalans, Divi-divi, Bablah fruits, Pomegranate, Tea, Coffee, Oak, Willow, Elm, Fir, Birch, Acacia, Male-fern, Cinchona, Cinchona-nova, Ipecacuanha, Mate and Celastrus; Morin-tannic, Gallo-tannic, Leditannic and Nucitannic Acid, p. 156.

OTher Glucosides

$\S 166$. Cyclopin, Rhinanthin, p. 163.-\$167. Solubility; Description of the more important Glucosides. Amygdalin and Laurocerasin, Estimation; Myronic Acid, Estimation; Sinalbin (and Sulphocyanate of Sinapine), Menyanthin, Pinipicrin, Coniferin, Arbutin, Daphnin, Salicin, Populin, Benzohelicin, Philyrin, Phlorrhizin, Asculin, Fraxin, Syringin, Globularin, Pittosporin, Samaderin, Colocynthin, Bryonin, Ononin, Apiin, Datiscin, Physalin, Dulcamarin, Hesperidin, Crocin, Glycyr- 
rhizin, Panaquillon, Thevetin, Chamælirin, Gratiolin, Paridin, Convallarin, Convallamarin, Helleborin and Helleborein, Scillaïn, Saponin, Digitonin, Senegin, Melanthin, Parillin, Sapogenin, etc., Indican, Indigo-blue, p. 164.-\$ 168. Nonglucosidal Bitter Principles, Cusparin, Chinovin, Cnicin, p. 175. -\$169. Aloins, p. 176. - $\$ 170$. Carthamin, p. 178.

AlKalords

$\S 171$. Colour Reactions of the more important Alkaloids, p. 178. - $\$ 172$. Identification, p. 181. - $\$ 173$. Double Chlorides with Gold and Platinum, p. 181.- $\$$ 174. Further Remarks on Titration with Potassio-mercuric Iodide; Atropine, Hyoscyamine, Coniine, Strychnine and Brucine, Morphine, Narcotine, Chelidonine, Veratrine, Sabadilline and Sabatrine, Calabarine and Physostigmine, p. 182.-\$ 175. Estimation of Coniine with Phosphomolybdic Acid; of Pilocarpine; Application of Phosphotungstic Acid, Tannic Acid, Picric Acid in the Estimation of Alkaloids, p. 184.- $\$$ 176. Determination of Alkaloid in Tea, Coffee, Guarana ; Lieventhal's and Claus's Methods, p. 186. - $\$ 177$. Estimation of Theobromine in Cacao; Methods of Trojanowsky and Wolfram, p. 187.- $\$ 178$. Estimation of Piperine, p. 188. - $\$ 179$. Volumetric Estimation of Nicotine, p. 188. - $\S$ 180. Estimation of Coniine, p. 189.$\S 181$. Separation of two or more Alkaloids from one another; Jervine and Veratroidine, Paricine, Narceïne and Narcotine, Morphine and Codeïne, Morphine and Narcotine, Strychnine and Brucine, p. 189.- $\$ 182$. Separation by Solvents; Strychnine and Brucine; Colchicine and Colchiceine; Cinchonine and Amorphous Alkaloid; Delphinine and Delphinoïdine; Morphine and Narcotine; Morphine, Codeïne, and Thebaïne; Delphinine, Delphinoidine and Staphisagrine, p. 191.—§183. Separation of Quinine and Cinchonidine from other Cinchona Alkaloids; of Quinidine from Cinchonine; of Quinine from Cinchonidine; of Strychnine from Brucine: of Calabarine from Physostigmine; of Chelidonine from Sanguinarine; of Muscarine from Amanitine ; Paytine, etc., p. 193.- $\$ 184$. Separation of the more important Cinchona Alkaloids from one another, p. 194.- $\$ 185$. Estimation of Cinchona-Alkaloids by Polarization, p. 198.-\$186. Rarer Cinchona-Alkaloids; Aricine, Cusconine, Quinamine ; Paricine, Paytine, p. 198.— $\$ 187$. Estimation of the more important Opium-Alkaloids, p. 199. - 188. Methods of Procter, Prollius, Fluickiger, p. 200.$\S 189$. Other Alkaloids ; Ergotinine and Picrosclerotine ; Curarine, Erythrophlœine, Lobeliine, Conessine, or Wrightiine, Harmaline and Harmine, Surinamine, Aribine, Atherospermine, Rhœadine, Violine, Beberine, Belladonnine, Cocaine and Hygrine, Chlorogenine and Porphyrine, Corydaline, Cytisine, Ditamine, Geissospermine, Aspidospermine, Dulcamarine, Glaucine, Fumarine, etc., p. 201.-\$190. Amanitine, Muscarine, Choline, Betaine, p. 205.-\$ 191. Asparagine, Glutamine and Estimation of the Eame, p. 206.-\$ 192. Leucine, Chenopodine, Tyrosine, Rhatanhin, p. 207.

Vegetable Mucilage •

$\S$ 193. Differences in Gum and Pectin, p. 208.-\$ 194. Modified 
Method of Examination for Gum, p. 209.- $\$ 195$. Characters of Soluble Vegetable Mucilage (Arabin, Arabic or Gummic Acid); Metarabic Acid, p. 210.—§196. Behaviour to Reagents ; Commercial Varieties of Gum-arabic, p. 211.- $\$ 197$. Separation of Arabin from Dextrin, Glucose, Saccharose, etc., p. 212.

Dextrin, Triticin, Levulin, etc.

$\S$ 198. Distinctive Characters, p. 212.- $\$ 199$. Formation of Alcoholates; Composition; Estimation by Titration and Polarization, p. 213.

Glucoses

$\S 200$. Detection of Grape-sugar; Reactions to distinguish Grape-sugar from Cane-sugar, Milk-sugar, Mannite, etc., p. 214. - $\$ 201$. Detection and Estimation in Presence of Dextrin, p. 215. - $\$ 202$. Detection of Dextrin in Presence of Cane-sugar, p. 215. - \$ 203. Estimation of Glucoses in Presence of Cane-sugar, p. 215. - $\$ 204$. By Fermentation; Influence of Substances retarding Fermentation, p. 216. - $\$ 205$. Characteristic Properties of Grape-, Fruit-, Invert-, Salicin-, and Caragheensugar ; Phlorose, Arabinose, Galactose, p. 217.-\$ 206. Inosite Sorbin, Eucalyn, Nucite, p. 219.—§ 207. Cane and Milk-sugar; Maltose, Melitose, Melezitose, Mycose, p. 220.—§ 208. Estimation of Glucoses and Saccharoses by Polarization, p. 221.$\S 209$. Estimation of Two Glucoses by Titration and Polarization, p. 222. - $\$ 210$. Estimation of Cane- and Invert-sugar, p. 223.- $\$ 211$. Estimation of Three Sugars in Solution together, p. 224. - \$ 212. Mannite, Dulcite (Melampyrite), Isodulcite (Rhamnodulcite), Hesperidin-sugar, Sorbite, p. 224.$\S$ 213. Mannitan, Quercite, Pinite, Abietite, p. 225.

$\S 214$. Reactions of Malic Acid; Separation from Oxalic,

Tartaric, Citric, Succinic, Gallic, Tannic, Benzoic, Acetic, Formic Acid, p. 214.- $\$ 215$. Estimation of Citric Acid as Barium-salt, p. 206. - $\$ 216$. Reactions of Citric Acid; Aconitic Acid, p. 227.- $\$ 217$. Estimation of Tartaric Acid as Acid Tartrate of Potassium, p. 228.- $\$ 218$. Estimation of Tartaric and Citric Acid when present together; Separation from Malic, Oxalic, Phosphoric, and Sulphuric Acid ; Racemic Acid, p. 228. -\$219. Oxalic Acid; Separation from Tartaric and Citric Acid; Isolation from Oxalate of Calcium, p. 230.$\S 220$. Succinic Acid; Separation from Oxalic, Tartaric, and Citric Acid, p. 230.-§ 221. Fumaric and Maleic Acids; Kinic Acid; Rubichloric Acid, p. 232.-§ 222. Lactic Acid, p. 232. - $\$ 233$. Glycolic Acid, p. 233.

ALBUMINoIDs, ETC.

$\S 224$. Calculation of Nitrogen into Albuminoids, p. $234 .-\S 225^{\circ}$. Repetition of Estimation of Legumin, p. 234.-\$226. Caseïn, Glutencaseïn, Fibrin ; Globulin, p. 235.-\$227. Vitellin, p. 236. - $\$ 228$. Myosin, p. 236. - $\$ 229$. Estimation of Albuminoids by Titration with Tannin, p. 236. - $\$ 230$. Comparison of Results with those of the Estimation by Coagulation; Ferments ; Diastase, Invertin, Emulsin, Myrosin, Papayotin, etc., p. 237.- $\$ 231$. Estimation of Albuminoids with Acetate of 
Copper, p. 238.- $\$ 232$. With Acetate of Lead, p. 238.—§ 233. Estimation of Albuminoids Soluble in Dilute Acid; Albuminoids capable of being assimilated, p. 240.—§234. Albuminoids Soluble in Spirit; Glutenfibrin, Gliadin, Mucedin, p. 241.$\S 235$. Properties of the same, p. 242. $\$$ 236. Gluten; Estimation, p. 243.-\$237. Albuminoids precipitated simultaneously with Metarabic Acid, etc., p. 243. — $\$ 238$. Nitrogenous Substances Insoluble in Water, Dilute Acid, and Dilute Alkali, p. 244.

Amine Compounds . .

$\S 239$. Distinctive Characters of Monamines, Diamines, etc., p. 244. $\S 240$. Separation of Ethyl- and Methyl-amine from the corresponding Di-and Tri-amines, p. 244.-\$ 241. Approximate Estimation of Amides, p. 245. - $\$ 242$. Cathartic Acid, Sclerotic Acid, Scleromucin, Assay of Rhubarb, p. 247.

Starch, Lichenin, Wood-gum, etc. .

$\S 243$. Constituents of Starch, p. 249. - $\$ 244$. Constituents of the

Cell-wall that turn Blue with Iodine; Lichen-starch, p. 250.$\S 245$. Lichenin and Gelose, p. 251.—§ 246. Wood-gum, p. 252. Cellulose, Lignin, and Allied Substances

$\S 247$. Researches of Frémy and Terreil on Composition of Woodytissue ; Cuticular and Incrusting Substances; Modifications of Cellulose, Lignin (Vasculose, Incrusting Substances), Suberin, Glyco-lignose, Glyco-drupose, p. 252. $-\$ 248$. Composition of Cellulose, p. 256. $-\$ 249$. Properties of the Various Forms of Cellulose, p. 256. - $\$ 250$. Crude Fibre ; Estimation, p. 257.

PERCENTAGE COMPOSITION OF THE CONSTITUENTS OF PLANTS REFERRED TO

COMPOSITION OF THE MORE IMPORTANT CONSTITUENTS OF PLANTS ARRANGED ACCORDING TO THE PER. CENTAGE OF CARBON 


\section{PLANT ANALYSIS :}

\section{QUALITATIVE AND QUANTITATIVE.}

\section{INTRODUCTION.}

$\S 1$. AN accurate qualitative and quantitative analysis of a plant or vegetable substance is not unfrequently referred to as one of the most difficult tasks that a chemist may be called upon to undertake. Attention is very properly directed to the great number of species of plants that occur in nature, to the great abundance and variety of their chemical constituents, and to the circumstance that almost every skilful analysis of a plant that has not previously been examined yields new, hitherto unknown products. Prominence is also justly given to the fact that the analysis of vegetable substances differs from that of minerals, inasmuch as the elements present in the latter have in many instances only to be separated and weighed or measured, either as such or in the form of certain of their simpler, more easily recognisable compounds, whilst in the analysis of plants it far more frequently occurs that the proximate principles themselves must be first separated before they can be examined or weighed. These reasons are all admissible; we are, moreover, justified in pointing out, amongst other numerous difficulties encountered in the analysis of plants, the great proneness to decomposition of many of the constituents of vegetable substances and the errors that may arise therefrom, not only in the estimation of these bodies themselves, but also of such substances as may accompany them. But surely these considerations should not tend to prevent investigations from being carried out :whioh. are equally

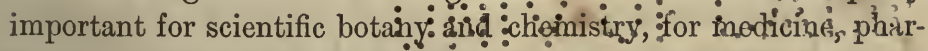
macy, dietetics, agriculture etc. By systematically arranging : 
the methods of examination hitherto devised, either for the estimation of a single constituent or for the separation of several substances contained in a plant, I hoped to succeed in inducing others to conduct investigations in a department of chemistry at present so much neglected; and it was in that hope that I decided upon the compilation of this work. In it I trust to be able to show that for the separate estimation of many substances we have methods at our disposal which, in point of accuracy, are nearly $\backslash$ abreast of the processes employed for the determination of mineral constituents, and that we can often obtain results really serviceable in the investigation of the more important component substances contained in a plant. I especially hope to succeed in showing that analyses of plants possess in one respect an advantage over the analyses of minerals, inasmuch as it often happens, in examining mixtures or conglomerates of several chemical individuals, that in the latter case a much less satisfactory insight into the constitution can be obtained than in the former. The elements, for instance, of which a granite is composed can easily be determined by inorganic analysis, but it is exceedingly difficult to ascertain with exactitude in what quantity each separate mineral occurring in the granite is present. But in the analysis of vegetable substances the endeavour is made from the outset to separate the different chemical individuals from one another, and by the use of various solvents this is frequently possible. In this respect, therefore, the analysis of a plant can often be made more complete than that of a mineral.

$\S 2$. The object that I have sought to attain in this work was the compilation of a method of analysis applicable to the qualitative and quantitative examination of vegetable substances of both known and unknown composition, and of an introduction to the qualitative and quantitative determination of the various more important constituents of plants with which we are at present acquainted.

I need scarcely observe that I have given the fullest possible consideration to the question as to which tissues of the plant contain the various constituents, and have therefore, for that purpose, made use of microchemical analysis.

With reference to the "arraigernon of the matter in the work, I would remark that in "the mothod of analysis contained in 
Part I., I have not separated the qualitative and the quantitative determinations of the more important substances from each other. I have made the method of separation serve as a leading principle, and have therefore grouped together the constituents of plants in such a manner that all those may be considered together that are isolated by the same means. I have then placed in sub-divisions of the principal groups such substances as may be isolated by special methods, and these latter are also discussed.

The more important peculiarities of the various bodies belonging to the different groups, as well as special methods for the estimation of some of them, have been placed in Part II., which has been so arranged as to follow closely on Part I. in the form. of a supplement. In this way I hope to be more easily able to avoid repetition, and especially to facilitate investigations in which the substances that may be found are unknown. Thus a method of analysis, taking account of the more important constituents of plants, may be traced through the work.

$\S 3$. It has always been accepted, as an important principle, by those who have been engaged in plant analysis, that the constituents present should be separated as far as possible by means of different solvents. I have also followed this plan, which has in many instances proved itself adapted to the attainment of the object in view, and I concur with those chemists who recommend the use, as far as practicable, of the most indifferent solvents. If, in the analyses of vegetable substances I have already made, I have deviated from the course followed by my predecessors, ${ }^{1}$ I have done so, first, in increasing the number of solvents; and secondly, in varying the order in which those solvents were allowed to act upon the substances under examination. I shall subsequently show that this may have a great influence on the result of the analysis.

${ }^{1}$ I draw particular attention here to Rochleder's 'Anleitung zur Analyse von Pflanzen und Pflanzentheilen' (Würzburg, 1858), which I regard as opening up new ground in this subject. See also Wittstein, 'Anleitung zur chemischen Analyse von Pflanzentheilen' (Nördlingen, 1868), and an English translation of the same by Baron von Mueller, "The Organic Constituents of Plants and Vegetable Substances and their Chemical Analysis' (Melbourne, 1878); Arata, 'Guja Paralel Análysis immediato de los Vejetales' (Buenos Aires, 1869); and a paper by Parsons in the American Chemical Journal, vol. i. No. 6 . 
It will be seen from the foregoing that the principal groups into which I have divided the matter to be treated are formed by the behaviour of the plant constituents to solvents.

In a chapter preceding the method of examination proper, I have given a few general rules for plant analysis. 


\section{METHOD OF ANALYSIS FOR THE MORE IMPORTANT CONSTITUENTS OF PLANTS.}

I.

Preliminary Operations. Estimation of Moisture AND AsH.

§ 4. Drying.-In the majority of cases the parts of plants at our disposal for analysis have already been dried, and we can only take account of the small amount of moisture that has been absorbed from the air in consequence of the hygroscopic nature of the vegetable tissue in contact with it. I can only recommend that the estimation of moisture, for which a temperature not exceeding $110^{\circ}$ will as a rule suffice, be made with a small quantity of the substance. I should not advise the drying of the material intended for use in the investigations to be discussed in the following chapters, because, even at a temperature of $100^{\circ}$ to $110^{\circ}$, a number of constituents prone to decomposition undergo chemical change. It will be sufficient if the moisture be estimated in about 2 to 5 grams, that is, if that quantity be kept at the temperature indicated till it ceases to lose weight. By means of this determination the results of all other estimations can be calculated to the dry substance. ${ }^{1}$

${ }^{1}$ An apparatus for drying material for agricultural (chemical) analysis has been described by Hugo Schulz (Landw. Versuchsstat, vol. ix. p. 213); one for the rapid estimation of water in organic substances by Gawalovski in the Zeitschrift f. anal. Chemie, xiii. 267 (1874). For the determination of moisture in fruits rich in sugar, such as apples, etc., Tschaplowitz (ibid. Jg. 19, p. 243, 1880), recommends the slices to be first extracted with absolute alcohol containing 10 to 20 per cent. of ether, and then dried at $100^{\circ}$ to $110^{\circ}$, the ether-alcohol solution to be evaporated, the residue heated to $85^{\circ}$ to $90^{\circ}$ and then added to the dry substance. See also Reischauer in the Jahresb. f. Pharm. Jg. 1867, p. 8 (Amer. Journ. Pharm. xxxviii. 74) ; Schoonbroodt, 
That portion which has served for the determination of the moisture can subsequently be used for the estimation of the total ash.

$\S 5$. Treatment of Fresh Plants.-If fresh plants or parts of the same are to be examined it will be advisable in many cases, at least if a quantitative examination is to be made, to first dry the material, or it will at any rate be necessary for those portions which are subsequently to be treated with petroleum spirit, ether, alcohol, and similar menstrua. Here, too, it will be desirable to make an accurate estimation of the moisture, and in doing so it is advisable to allow the temperature to rise very gradually to $100^{\circ}$ or $110^{\circ}$. The greater part of the material can as a rule be dried at a temperature under $30^{\circ}$ till in a condition suitable for powdering, and the amount of moisture still retained in it can be determined in a small portion by a separate estimation. In drying fleshy fruits or roots care should be taken not to reduce them to too fine a state of division. Leaves which are not too fleshy do not require any preparation at all. It is very desirable that as little of the cell-tissue as possible should be deprived of its natural covering, as by doing so the action of the air on the decomposable constituents is only facilitated. With substances which are very, rich in sugar it is better not to dry the portions destined for the estimation of the saccharine matter at all, but to examine them in the fresh state. The same holds good for such substances as are very rich in ethereal oil, or contain volatile acrid compounds; I shall subsequently show that such compounds may be easily isolated from, and determined in, the fresh plants. Of course the amount of such volatile substances as may be found by other means must be deducted from the result of the determination of moisture.

$\S 6$. Powdering.-It is of the greatest importance that the material for the various estimations should be uniformly mixed and reduced to the very finest powder possible. It may be asserted that the greatest errors made in the analysis of plants are due to the material not having been reduced to a sufficiently fine state of subdivision. Estimations of oil made with ether or petroleum spirit often show differences of several units per cent.,

ibid. Jg. 1869, p. 9. (Pharm. Journ. Trans. [2], xi. 84). In the latter work illustrations are given of the difference in composition that may be met with in fresh and dried, and in quickly and slowly dried, vegetable substances. 
because these solvents do not penetrate into the cells, but only dissolve that which is adhering to the external surfaces of the object. It must be admitted that it is often very difficult to reduce a vegetable substance to an impalpable powder, but the necessity of sparing no trouble in this respect must be most strongly urged. It may sometimes be expedient to dry very hard substances, such as seeds, etc., at $100^{\circ}$ to $110^{\circ}$ before powdering them. Coffee-seeds may thus be reduced to quite a fine powder, especially if triturated in an agate mortar with a known quantity of powdered glass or sharp sand (that has been previously treated with hydrochloric acid). Somewhat hard substances may occasionally be grated upon a fine grater with advantage, and then powdered as above. Tough material, too, and such as is to be examined in the fresh state, may be generally prepared in this way. In working with substances containing much fixed oil it is sometimes expedient to dry the residue after the first extraction with petroleum spirit, etc., powder it again and repeat the extraction.

§ 7. Estimation of $A$ sh. - With regard to the total ash, which is usually estimated in plant analysis, reference may be made in the majority of cases to the generally known methods of procedure. For vegetable substances that are very difficult to incinerate, it is advisable, after carbonization, to cool, powder as finely as possible, and continue the heating, placing a cylindrical tube vertically above the platinum dish, so as to create a current of air. Or the incineration may be conducted in a Hempel's jacket with access of air. If easily fusible salts are present and prevent complete incineration, the admixture of about an equal weight of nitrate of ammonium with the cooled mass, and repeated ignition, may render good service. Or the carbonized mass may be mixed with a weighed quantity of oxide of iron, and the incineration continued. ${ }^{1}$

After weighing the ash the quantity of carbonic acid present in it is to be determined and deducted from the total weight. The carbonic acid is simply a part of the organic matter, the rest of which has been burnt off, and is to be determined in other ways. It is also desirable to test the ash for sand, and finally, if a complete analysis is not required, to estimate at least the total quantity of phosphoric and sulphuric acid and potash. (See also § 82).

${ }^{1}$ Compare also Bornträger, Zeitschr. f. anal. Chemie, B. xvii. p. 440 (1878). 
II.

Examination of the Substances Soluble in Petroleum SPIRIT.

ETHEREAL AND FATTY OILS, WAX, ETC.

§ 8. Petroleum Spirit.-I have proposed the use of petroleum spirit in the analysis of plants on account of its being a relatively good solvent for most ethereal and fatty oils, but not for the majority of resins and allied substances which would have been simultaneously brought into solution had ether been used. We have therefore in this liquid a means of more accurately estimating ethereal and fatty oils than was formerly possible with ether. Another advantage which petroleum spirit possesses over ether is that it does not, like ether, cause a coagulation of soluble albuminous compounds in substances rich in such bodies. As it is desirable to deprive the material of fat before extracting the soluble albuminous substances for their quantitative determination, the whole or part of the residue after treatment with petroleum spirit may be very well employed for this purpose. A chief condition for the successful application of petroleum spirit is that it be very volatile. It must therefore be purified by repeated fractional distillation, and care taken that it contains no compound boiling above $45^{\circ}$. It is, moreover, desirable to distil it over fat (lard) to free it from some of the impurities of more powerful odour.

§ 9. Extraction with Petroleum Spirit.-It has already been mentioned in $\S 6$ that vegetable substances to be extracted with petroleum spirit must be reduced to the finest powder possible. It is advisable in such extractions to employ a known quantity of petroleum spirit-say five to ten times that of the substance to be treated; or, better still, for every gram of the latter $10 \mathrm{cc}$. of the former. A small narrow cylinder with glass stopper may 
be used for this purpose. It should be weighed immediately after the introduction of substance and menstruum; or, if graduated, the volume only occupied by both need be noted. They may be macerated for about eight days, shaking several times daily, and then made up to the original volume or weight by the addition of petroleum spirit, to replace any that may have been lost by evaporation. This having been done, it is sometimes only necessary to evaporate an aliquot part of the solution, and calculate from the residue the weight of the substances which have been brought into solution. ${ }^{1}$

The supernatant liquid frequently becomes so perfectly clear on standing, that all trouble of filtration may be avoided by removing with a pipette a definite volume, which may then be evaporated and weighed. ${ }^{2}$

This method of procedure is especially to be recommended if the object under examination contains ethereal oil, in which case all washing of the residue, or any dilution whatever of the petroleumspirit solution, should be carefully avoided. The more concentrated the petroleum-spirit extract is, the more accurate will be the gravimetric estimation of the ethereal oil. If, however, the petroleum-spirit solution is to be filtered off and the residue on the filter washed, care should be taken that a funnel with ground edges be employed and kept well covered.

For the evaporation of the petroleum-spirit solution no porcelain basin or round-bottomed platinum or glass dish should be used, on account of the loss easily caused by the capillarity of its sides. It is expedient, as a rule, to use a flat-bottomed glass dish with vertical sides and well-ground edges, a ground-glass plate acting as a cover. If the presence of a rapidly resinifying oil is suspected, the petroleum-spirit solution may be evaporated in a tared flask by passing a current of carbonic acid gas through it whilst kept surrounded with warm water. (See also § 138.)

${ }^{1}$ In this case, a slight error is introduced into the calculation, by the increased volume of the petroleum spirit due to dissolved oil. But this will, as a rule, be so small that it may be entirely neglected; or, if desirable, a correction may be made after weighing the residual oil, since we know that the specific gravity of the fatty oils hitherto examined ranges from 0.91 to 0.925 .

${ }^{2}$ Even when the petroleum-spirit solution does not become quite clear on standing, as is often the case when seeds are under examination, it is better to measure off a quantity with a pipette, filter it, and wash the filter and the mouth of the funnel (on the outside) with petroleum spirit, than to filter off the whole of the liquid and measure off a quantity for evaporation. 
Shallow evaporating dishes, which can be enclosed between clamped glasses and weighed, may also be used if ethereal oil is present; but they must be placed in other larger dishes during the evaporation of the petroleum spirit. It is, however, preferable even in these cases to use the glass dishes with vertical sides previously described.

$\S 10$. Treatment of Fresh Plants.-Fresh, very aromatic parts of plants may be examined as stated in $\$ 5$, without being previously dried. ${ }^{1}$ They should be as finely divided as possible by pressure and trituration, then packed in a small percolator, and the moisture present displaced by the smallest possible quantity of petroleum spirit or ether; the latter is, perhaps, in this case to be preferred. The menstruum itself must subsequently be displaced by water. The liquids may be received in a graduated burette fitted with a glass stop-cock and long fine point; in this the ether or petroleum spirit may be allowed to separate, and an aliquot part measured off for evaporation. (See also $§ 22$ and following.)

\section{EXAMINATION OF THE FIXED OIL.}

§ 11. Detection and Estimatim. - We will first consider the simpler case in which the petroleum spirit (or ether) dissolves fixed but not ethereal oil. The absence of the latter may be recognised by the light colour of the petroleum-spirit solution and its residue after evaporation, and by the absence of any aromatic odour which would otherwise be given off during the evaporation of the last traces of solvent, the operation being conducted at the ordinary temperature. That we really have a fixed oil to deal with may be shown by the uniform character of the spot left on evaporating a drop of the petroleum-spirit solution on a sheet of blue notepaper.

On examining vegetable substances under the microscope, fixed oil is seen in the form of small globules of high refracting power, which dissolve in petroleum spirit, ether, and bisulphide of carbon, and are saponified by a dilute solution of soda. If the objects examined are fresh it is advisable to treat the section with a relatively large quantity of water. Concentrated solutions of sugar and similar substances have the power of dissolving oil, which is, however, again separated on the addition of a large

i For information concerning the so-called dietheralysis, see Legrip, Union Pharm. V. vi. p. 65 (1876). 
quantity of water. "I do not think it improbable that in the juice of fresh plants oil is held in solution by carbohydrates and does not show itself until separated by dilution with water. And in examining the expressed juice of fresh plants, or concentrated infusions of the same, it is well to bear this peculiarity of oils in mind.

To determine the total amount of fixed oil, the residue from the evaporation of part or all of the petroleum-spirit solution is dried at $100^{\circ}$ till the weight remains constant, which may then be noted. For further information respecting the estimation of fixed oils, and especially the apparatus to be used, see $\S 120$. Compare also $\$ 36$.

The fatty residue so obtained may be kept for some time, to observe whether partial or complete solidification does not gradually take place. The solubility in absolute alcohol, spirit of 95 and 90 per cent., may also be tested, to ascertain whether free fatty acids, cholesterin, resinous bodies, caoutchouc, or such compounds, can be isolated. (Cf. $\$ \S 125,126,127,130$.) It may also be observed whether the oil is easy or difficult to saponify, whether the soap is soft or hard, colourless or coloured, whether glycerine is separated during saponification, and the fat consequently contain glycerides (cf. § 13), and whether the oil resinifies readily on exposure to the air ( $\$ 121)$. Finally, the melting and solidifying points may be taken. Concerning this determination see $\S 17$.

$\S 12$. Composition.-If a further insight into the composition of the fixed oil is required, larger quantities must be prepared either by extraction, or by expression followed by extraction, according to the nature of the material and the quantity of oil it contains.

A few qualitative experiments may first be made with a portion of this oil. If it remains fluid at ordinary temperatures the action of nitrous acid may be tried. The solidification of the oil would prove the presence of oleic $(\$ \$ 19,130)$ or an allied acid capable of conversion into elaidin ( $\$ 122)$. In this case, on mixing the oil with about one-fifth of its volume of concentrated sulphuric acid, but little heat will be evolved, whilst compounds of the drying linoleic acid $(\S 130)$ and its allies generally cause a considerable rise in temperature ( $\$ 123)$. For comparison parallel experiments may be made with linseed and almond or 
olive oil. Any colouration produced by the first drops of sulphuric acid should be noted, and the experiment repeated with a small quantity of the oil, adding a little syrupy phosphoric acid. The behaviour of the oil to syrupy chloride of antimony, nitric acid (from $\frac{1}{3}$ to 1 volume) of specific gravity $1 \cdot 3$, alone or combined with a little powdered sugar, may be tested. The action of concentrated solution of bisulphide of calcium, borax, and chloride of lime may also yield reactions characteristic of certain oils. (See $\$ 124$.) It may finally be ascertained whether the oil combines quickly with oxide of lead, and whether the plaster so produced is soft or hard, soluble or insoluble in ether.

If the fatty oil is solid at ordinary temperatures, a portion may be melted, and the above tests with acids, etc., applied. The solubility in ether should be tried, and note taken whether a solution in two parts of warm ether deposit sulid matter on cooling.

If the fixed oil from a vegetable substance partially solidifies after standing several days, the liquid part may be separated from the solid by filtration and expression, and each treated separately.

\$ 13. Composition; Estimation of Glycerine.-It is well known that natural fats are almost invariably mixtures of different glycerides or ethereal salts. If the various constituents of which a fixed oil is composed are to be ascertained, larger quantities (250 to 500 or 1000 grams) must be saponified with a solution of caustic soda of specific gravity 1.25 to 1.3 ; and after complete saponification, as shown by the soap dissolving in water warmed on the steam bath without the separation of undecomposed oil, the soap so formed may be thrown out by the addition of a concentrated solution of salt. The separation may be performed with advantage in tall beakers, which should be placed on the waterbath until the soap has assumed. such a condition that on cooling it can be removed as a solid cake. (See also $\S 15$.)

A measured portion of the aqueous liquid, after the removal of the soap, may be concentrated on the water-bath, or preferably at a temperature of $70^{\circ}$ to $80^{\circ}$, and the residue treated with absolute alcohol, or better with a mixture of about three volumes of absolute alcohol to one to two of ether, which dissolves the glycerine liberated by the decomposition of the oil. On evaporating this solution the glycerine remains behind as a sweet syrupy 
liquid. It is optically inactive, and yields acrolein when heated with acid sulphate of potassium. If the soap, after removal from the liquid, is washed several times with solution of salt and the washings added to the liquid in the beaker, then the glycerine obtained as described may be weighed. The estimation is not free from error, but it permits of an approximately correct idea being formed of the quantity of glycerine contained in the fat. (See $\S 128$. )

$\S$ 14. Cetyl-, Cerotyl-, Melyl-Alcohol.-In solid fats, especially in the so-called vegetable wax, cetyl, cerotyl, or melyl may be present as bases instead of glyceryl, in which case the fat is much more difficult to saponify than it otherwise would have been and there is formed, in addition to the soap, a kind of alcoholate of the fatalcohol. If to such a mixture of soap and alcoholate solution of chloride of barium is added, a barium soap insoluble in alcohol and ether is generally precipitated, whilst cetyl-, cerotyl-, or melylalcohol is liberated and may be extracted with ether. Or the precipitation may be accomplished with acetate of lead (in the absence of oleic acid), and the wax-alcohol extracted by ether from the dried mass. (Cf. $\S \S 126,129$.) The melting-point (see $\S 17)$ and the ultimate analysis will show which of these alcohols has been isolated ( $\$ 129)$.

Vegetable wax frequently dissolves in boiling absolute alcohol, but separates out again on the addition of a little water, as a rule before the resins $(\$ 145)$.

$\S 15$. Volatile Fat-Acids.-In prosecuting the examination of the fat-acids the soap obtained in $\S 13$ is warmed and again decomposed with excess of hydrochloric acid, the mixture of fat-acids separated from the aqueous liquid, and washed repeatedly with water. If the odour of the mixture points to the presence of a volatile acid, this latter must be separated from the less volatile by distillation. The distillate should be saturated with soda, evaporated, the residue again decomposed with hydrochloric acid, and the fatty acids separated from the aqueous liquid. The possible presence of valerianic, caproic, caprylic, pelargonic, capric, and lauric (\$130), also angelic and methyl-crotonic acid must be borne in mind. They may be identified by their boiling-points, saturating power for bases, and composition. Of course the acid must be tested to ascertain if it is a mixture or not of several volatile acids separable by fractional distillation. (Cf. § 25.) 


\section{SUBSTANCES SOLUBLE IN PETROLEUM SPIRIT.}

§ 16. Less-rolatile Fat-Acids.-If no volatile acids are present, or after their separation by distillation, as directed in $\$ 15$, the less volatile fat-acids may be dissolved in alcohol and subjected in alcoholic solution to a fractional precipitation with acetate of magnesium. This salt precipitates members of the fat-acid series more easily than it does oleic acid and its homologues, and of the fat-acids proper of the $\mathrm{C}_{\mathrm{n}} \mathrm{H}_{2 \mathrm{n}} \mathrm{O}_{2}$ series, those standing highest in the series (i.e. containing the largest number of carbon-atoms) are precipitated first. The magnesium precipitates appear at first as soon as the acetate has been added, and in that case, after having been well shaken for some time, they may soon be filtered off. But subsequently it becomes necessary to add strong solution of ammonia, as well as the magnesium salt, to produce precipitation, and to allow the mixture to stand twelve to twentyfour hours in a cold place before filtering. The fractional precipitation is so contrived that each precipitate shall weigh about 1 to 5 grams, and this is continued till the tolerably strongly ammoniacal liquid yields no further precipitation on the addition of alcoholic solution of acetate of magnesium. Each precipitate must be well washed with alcohol and decomposed with hydrochloric acid. The fat-acid must be washed with water, dried, and crystallized once from boiling alcohol. After carefully drying the crystals the melting-point of each fraction must be taken. The acids are then recrystallized repeatedly from alcohol, and the melting-point again determined. (Cf. $\S \S 130$ to 131.)

$\S 17$. Determination of Melting-Point.-The following is the method I adopt when I have only a small quantity of the substance at my disposal. I place a minute portion on the surface of mercury contained in a small beaker. This is then introduced into a small cylindrical copper air-oven in such a way that it does not rest on the bottom, but remains three or four centimeters from it. To allow of careful observation of the substance during the experiment, I use as a cover for the airoven an ordinary bottle the bottom of which has been cut off. A cork, perforated for a thermometer, is then fitted into the neck. The thermometer is now introduced through the perforation into the mercury contained in the beaker placed just beneath, until the bulb is completely covered. In doing so it is desirable that some of the minute fragments of fat-acid, or other substance, be as near the bulb as possible. The whole is now heated over 
a small flame, so that the temperature rises about $1^{\circ}$ every two minutes. ${ }^{1}$

§ 18. Melting-Points of Fat-Acids.-The melting-points of the several fractions before and after purification are noted. If in the same fraction the same melting-point is observed on both occasions, or if the estimations show a difference of only $0.5^{\circ}$, the conclusion may be drawn with tolerable safety that the precipitate under examination contains only one fat-acid. The observed melting-point is then compared with those of the more important fat-acids, and the result arrived at confirmed, if possible, by ultimate analysis.

Experiments that have hitherto been made assign to capric acid a melting-point of $30.0^{\circ}$; lauric, $43.6^{\circ}$; myristic, $53.8^{\circ}$; palmitic, $62.0^{\circ}$; stearic, $69 \cdot 2^{\circ}$; arachic, $75 \cdot 7^{\circ}$.

Mixtures of two of these acids in certain proportions possess, as the investigations of $\mathrm{Heintz}^{2}$ have shown, a lower melting-point than either of the constituents. Heintz has also noticed that the mixture, on solidifying, crystallizes in a characteristic form, or remains amorphous, according to the proportion in which the two constituents are present.

Mixture of Stearic Acid. Palmitic Acid. 100

90

80

70

60

50

40

30

20

10

$0 \quad 100$

Mixture of

Palmitic Acid. Myristic Acid. ${ }^{3}$

$\begin{array}{rr}100 & 0 \\ 90 & 10 \\ 80 & 20\end{array}$

Melts at
$69 \cdot 2^{\circ}$
$67 \cdot 2^{\circ}$
$65 \cdot 3^{\circ}$
$62 \cdot 9^{\circ}$
$60.3^{\circ}$
$56 \cdot 6^{\circ}$
$56 \cdot 3^{\circ}$
$55 \cdot 1^{\circ}$
$57 \cdot 5^{\circ}$
$60 \cdot 1^{\circ}$
$62.0^{\circ}$

$62 \cdot 0^{\circ}$

$60 \cdot 1^{\circ}$

$58 \cdot 0^{\circ}$
Solidifies at

$62.5^{\circ}$
$60.3^{\circ}$
$59 \cdot 3^{\circ}$
$56.5^{\circ}$
$55 \cdot 0^{\circ}$
$54 \cdot 5^{\circ}$
$54.0^{\circ}$
$53.8^{\circ}$
$54.5^{\circ}$
-

$62 \cdot 5^{\circ}$

60.3

$56.5^{\circ}$

$55.0^{\circ}$

$54 \cdot 5^{\circ}$

$54 \cdot 0^{\circ}$

$53.8^{\circ}$

$54 \cdot 5$

$55 \cdot 7^{\circ}$
$53 \cdot 5^{\circ}$
Manner of Solidification. Crystalline scales.

Delicate crystalline needles. Amorphous, lumpy. Large crystalline lamellæ. Amorphous, wavy, dull. Very indistinct needles. Fine crystalline needles. Crystalline scales.

Crystalline scales.

Scaly and indistinct needles.

1 For further information about this determination see also Pohl, in Polyt. Centrbl. Jg. 1855, p. 165 ; Bergmann, in Kunst und Gewerbebl. f. Bayern. Jg. 1867, Januarheft; Buis, in Annalen d. Chem. und Pharm. xliv. p. 152; Wimmel, in Annal. der Physik. xxxiii. 121 (Am. Journ. Pharm. xli. 22, 430); Redwood, in Pharm. Journ. and Trans. [3], vi. 1009 (1876).

${ }^{2}$ Annal der Physik. xcii. p. 588 (Pharm. Journ. and Trans. [1], xv. 425) ; cf. ibid. Ixxxiv. 226.

${ }^{3}$ For particulars of the examination of a fat in which stearic, palmitic, and myristic acids were found, see Greenish in Pharm. Journ. and Trans. [3], x. 909. 
Mixture of

Palmitic Acid. Myristic Acid.

70

60

50

40

30

20

10

$0 \quad 100$

Mixture of

Myristic Acid. Lauric Acid.

\begin{tabular}{rr}
100 & 0 \\
90 & 10 \\
80 & 20 \\
70 & 30 \\
60 & 40 \\
50 & 50 \\
40 & 60 \\
30 & 70 \\
20 & 80 \\
10 & 90 \\
0 & 100 \\
\multicolumn{2}{c}{ Mixture of }
\end{tabular}

Stearic Acid. Myristic Acid. 100

90

80

70

$60 \quad 40$

40

50

60

70

80

90

0

20

30

40

50

60

70

80

90

00

10

20

030

$50 \quad 50$

$40 \quad 60$

$30 \quad 70$

$20 \quad 80$

$10 \quad 90$

$0 \quad 100$

Mixture of

Palmitic Acid. Lauric Acid.

\begin{tabular}{rr}
100 & 0 \\
90 & 10 \\
80 & 20 \\
70 & 30 \\
60 & 40 \\
50 & 50 \\
40 & 60 \\
30 & 70 \\
20 & 80 \\
10 & 90 \\
0 & 100 \\
\multicolumn{2}{c}{ Mixture of }
\end{tabular}
Stearic Acid. Lauric Acid.

100

90

80
0

10

0

0

0

60

70

80

00

0

Melts at Solidifies at

$54.9^{\circ} \quad 51.3^{\circ}$

$51.5^{\circ} \quad 49.5^{\circ}$

$47.8^{\circ} \quad 45 \cdot 3^{\circ}$

$47 \cdot 0^{\circ}$

$46.2^{\circ}$

$49.5^{\circ}$

$51.8^{\circ}$

$53.8^{\circ}$

$\begin{array}{cc}53 \cdot 8^{\circ} & - \\ 51 \cdot 8^{\circ} & 47 \cdot 3^{\circ} \\ 49 \cdot 6^{\circ} & 44 \cdot 5^{\circ} \\ 46 \cdot 7^{\circ} & 39 \cdot 0^{\circ} \\ 43 \cdot 0^{\circ} & 39 \cdot 0^{\circ} \\ 37 \cdot 4^{\circ} & 35 \cdot 7^{\circ} \\ 36 \cdot 7^{\circ} & 33 \cdot 5^{\circ} \\ 35 \cdot 1^{\circ} & 32 \cdot 3^{\circ} \\ 38 \cdot 5^{\circ} & 33 \cdot 0^{\circ} \\ 41 \cdot 3^{\circ} & 36 \cdot 0^{\circ} \\ 43 \cdot 6^{\circ} & -\end{array}$

Melts at

$69 \cdot 2$ 。

$67 \cdot 1^{\circ}$

$65 \cdot 0^{\circ}$

$62 \cdot 8^{\circ}$

$59 \cdot 8^{\circ}$

$54 \cdot 5^{\circ}$

$504^{\circ}$

$48.2^{\circ}$

$478^{\circ}$

$51.7^{\circ}$

$53.8^{\circ}$

$62 \cdot 0^{\circ}$

$59 \cdot 8^{\circ}$

$57 \cdot 4^{\circ}$

$54 \cdot 5^{\circ}$

$51 \cdot 2^{\circ}$

$470^{\circ}$

$40.1^{\circ}$

$38.3^{\circ}$

$37 \cdot 1^{\circ}$

$41.5^{\circ}$

$43.6^{\circ}$

$69 \cdot 2$ 。

$670^{\circ}$

$64 \cdot 7^{\circ}$

$43 \cdot 7^{\circ}$

$41 \cdot 3^{\circ}$

$45 \cdot 3^{\circ}$
Manner of Solidification. Extremely fine needles.

Amorphous, lumpy.

Large crystalline lamellæ.

Indistinct lamellæ.

Amorphous.

Long needles.

Crystalline scales.

Crystalline scales.

Very minute crystals.

A " "

Large crystalline lamellæ.

Amorphous.

Amorphous, frond-like.

Crystalline needles."

Scaly crystals.

Manner of Solidification.

Scaly crystals.

Distinct crystalline scales.

Rather less distinct crystalline scales.

Still less distinct crystalline scales; no needles or lamellæ.

Scaly crystallization commences; no trace of needles or lamellæ.

Amorphous, opaque.

Beautiful large crystalline lamellæ.

Crystalline lamellæ.

Indistinctly crystalline.

Amorphous, opaque.

Crystalline scales.

Crystalline scales.

Still distinct crystalline scales.

Somewhat less distinct cryst. scales.

Still less distinct crystalline scales.

Granular, indistinct crystalline scales.

Almost amorphous, opaque.

Beautiful large crystalline lamellæ.

Small crystalline lamellæ.

Indistinctly crystalline.

Amorphous.

Crystalline scales.

Crystalline scales.

Still distinct crystalline scales. 
Mixture of

Stearic Acid. Lauric Acid. Melts at 70

60 30 40

50

50

40

30

60

70

20

80

90

100
$62 \cdot 0^{\circ}$

$59 \cdot 0^{\circ}$

$55 \cdot 8^{\circ}$

$50.8^{\circ}$

$43 \cdot 4^{\circ}$

38.5 。

$41 \cdot 5^{\circ}$

$43 \cdot 6^{\circ}$
Manner of Solidification.

Distinctly granular and scaly.

Granular ; commencement of scaly crystallization.

Almost amorphous, slightly granular.

Amorphous, warty.

On the surface shining faces of small crystals.

Amorphous, warty.

Amorphous.

Crystalline scales.

Heintz also noticed that a mixture of three fat-acids could melt at a still lower temperature, even if the third fat-acid added possessed a higher melting-point than either of the others. A mixture of 30 parts of palmitic and 70 of myristic acid melts at $46.2^{\circ}$, and solidifies amorphous. To 20 parts of this mixture stearic acid was added in the following proportions, and meltingpoint and manner of solidification observed:

$\begin{array}{cc}\text { Stearic Acid. } & \text { Melts at } \\ 1 & 45 \cdot 2^{\circ} \\ 2 & 44 \cdot 5^{\circ} \\ 3 & 44 \cdot 0^{\circ} \\ 4 & 43 \cdot 8^{\circ} \\ 5 & 44 \cdot 6^{\circ} \\ 6 & 45 \cdot 6^{\circ} \\ 7 & 46 \cdot 0^{\circ} \\ 8 & 46.5^{\circ}\end{array}$

Manner of Solidification.

Amorphous.

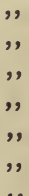

To 20 parts of a mixture of 30 parts of myristic with 70 of lauric acid, melting at $35.1^{\circ}$, palmitic acid was added, and the following observations made :

$\begin{array}{cc}\text { Palmitic Acid. } & \text { Melts at } \\ 1 & 33 \cdot 9^{\circ} \\ 2 & 33 \cdot 1^{\circ} \\ 3 & 32 \cdot 2^{\circ} \\ 4 & 32 \cdot 7^{\circ} \\ 5 & 33 \cdot 7^{\circ} \\ 6 & 34 \cdot 6^{\circ} \\ 7 & 35 \cdot 3^{\circ} \\ 8 & 36 \cdot 0^{\circ} \\ 9 & 37 \cdot 3^{\circ} \\ 10 & 38 \cdot 8^{\circ}\end{array}$

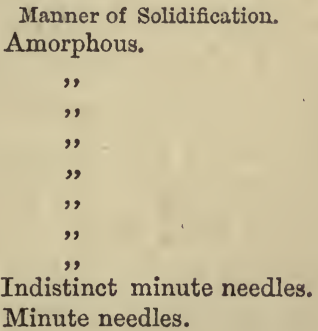

These tables show clearly that it is important to examine the fractions in the succession in which they were prepared. For instance, supposing the first precipitate to have yielded a fatacid melting at $68^{\circ}$, which might consequently be considered as stearic acid, the following precipitates fat-acids melting at about 
$56.6^{\circ}$, and subsequently one melting at $62^{\circ}$, the conclusion to be drawn is that the last is palmitic acid, and that the fractions with lower melting-points consist of mixtures of stearic and palmitic acids. According to Heintz's table a mixture of equal parts of stearic and palmitic acid should melt at $56 \cdot 6^{\circ}$, and assume on cooling a lamellar crystalline structure. Should no palmitic acid have been found, but in its stead a fat-acid melting at about $53^{\circ}$ to $54^{\circ}$, the presence of myristic acid is to be inferred and the mixture melting at $56.6^{\circ}$ would contain about 55 parts of stearic to 45 of myristic acid.

It is easy therefore to understand that if these observations be correctly interpreted a rough judgment may be formed of the smount of the separate acids present in the fat.

At the ordinary temperature pure stearic acid dissolves in about 40 parts of absolute alcohol, but in much less ether. When suspended in water it may easily be collected and removed by agitation with the latter solvent. The barium and calcium salts are soluble in boiling alcohol, but the major part separates out again on cooling.

Palmitic acid dissolves much more easily in warm and cold alcohol, and is very soluble in ether. It may also be collected when suspended in water by shaking with ether.

§ 19. Oleic Acid, etc.-The alcoholic liquid from $\S 16$, which gives no further precipitate on the addition of acetate of magnesium and ammonia, may be freed from alcohol by distillation under diminished pressure. That may be accomplished, both in this and many other cases, in the following manner: A retort is charged with the liquid, into which a few pieces of scrap platinum may with advantage be introduced, and attached to a Liebig's condenser provided with a tubulated receiver, care being taken that all connections are air-tight. The exhausting tube of a Bunsen's air-pump is then introduced into the tubulure of the receiver. Even if the evacuation be carried to only one-half an atmosphere, aqueous infusions, etc., may be rapidly concentrated on the water-bath and decomposition thus avoided which would otherwise easily be caused by overheating, or by the action of the air, etc.

After the recovery of the alcohol by distillation, the residue is poured from the retort, which may be rinsed with a little water, and acidulated with hydrochloric acid. The fat-acid which collects on the surface of the liquid may be removed mechanically, 
or by agitation with ether. In examining these acids attention must be paid to the possible presence of members of the oleic-acid series $(\$ \S 130,131)$ and of the allied ricinoleic acid. (See also $\$ 12$. As a preliminary operation an ultimate analysis may be made; and if this, as well as the reactions of the oil already observed, does nat point directly to a particular acid, an attempt must be made to accomplish a separation either by treating the plaster obtained by heating the fat-acid with oxide of lead, with ether (which dissolves oleate of lead) or absolute alcohol ; or by fractionally precipitating an alcoholic solution of a soda-soap with acetate of barium, or acetate or chloride of calcium (\$§ 130,131).

\section{CHLOROPHYLL AND ALKALOIDS EXTRACTED SIMULTANEOUSLY} WITH THE FIXED OIL.

§ 20. Chlorophyll.-The petroleum-spirit extract of vegetable substances often shows a green colour by transmitted light. This is generally due to chlorophyll. Such solutions are usually fluorescent, and appear blood-red by reflected light. Pure chlorophyll is only slightly soluble in petroleum spirit, and its presence in this extract is accounted for by the influence exercised apon its solubility by the fixed oil. That the green colour is really due to chlorophyll may easily be shown by spectroscopic examination. White light, on passing through a solution of this substance, undergoes a change in various of its constituent colours, as shown by the absorption bands in the spectrum. If the Fraunhofer line A correspond to 17 on the scale, B to 28, $\mathrm{C}$ to $34, \mathrm{D}$ to 50 , and $\mathrm{F}$ to 90 , there are observable in the spectrum (compare Table I. to $\S 148$, Nos. 13 and 14) ${ }^{1}$ four absorption bands situated between $\mathrm{B}$ and $\mathrm{F}$, the darkest of which extends from 30 to 42 , and the remaining three from 44 to 50,52 to 56 , and 58 to 60 respectively. From 80 to the end the spectrum gradually darkens. Of these absorption bands only the first two can be observed in dilute solutions, and the relative amount of chlorophyll dissolved may be judged from the presence or absence of the others. It would be scarcely possible to obtain absolute values for the amount of chlorophyll present, as liquids containing but very small quantities of that body are comparatively deeply coloured. Moreover, no method has hitherto been found available

I In examining a fresh leaf, only the most marked line between $\mathrm{B}$ and $\mathrm{C}$ is seen. Compare Vogel, Ber. d. d. chem. Ges. B. xi. pp. 623, 1367 (1878). 
for separating chlorophyll from the substances that accompany it. But if series of analyses are to be made with the same plant, to determine the changes it undergoes under the influence of the seasons, or certain conditions of cultivation, etc., the relative quantity of chlorophyll may be estimated by the ontical (colorimetric) method. It is better, however, to use alcohol or ether instead of petroleum spirit, as the latter does not usually extract the whole of the chlorophyll present. Admixture of foreign colouring matter may be avoided by first extracting the material several times with water, and drying the residue at the lowest temperature possible. The chlorophyll may then be dissolved out by alcohol or ether. (See further in $\S \S 37,132$. )

Under the microscope chlorophyll is seen to be associated with semi-fluid substances allied to protoplasm, often in the form of small granules (the so-called chlorophyll-granules), from which it may be extracted by alcohol. It is more rarely found equally distributed throughout the whole of the protoplasm covering the inner surface of the cell wall. It is bleached by chlorine and eau de Labarraque; the green colour is changed to yellow by dilute acids, and blue by concentrated hydrochloric acid.

$\S 21$. Alkaloids extracted by Petroleum Spirit.-Parts of plants containing alkaloid may, when extracted with petroleum spirit, yield some of the alkaloid, together with fixed oil, to that menstruum, even when the pure alkaloid is insoluble in it. Here, too, it is the fixed oil that determines the solution of the alkaloid. The presence of the latter may be detected by evaporating the petroleum-spirit solution, shaking the residue with water acidulated with sulphuric acid, and separating the aqueous from the oily liquid. Should an emulsion have been formed, separation may be induced by allowing the mixture to stand at a temperature of $40^{\circ}$ to $50^{\circ}$. The last traces of suspended fat may be removed from the acid liquid by shaking with petroleum spirit, and the presence of alkaloid demonstrated by the usual reagents. (Cf. $\$ 63$.) The amount will not often be large enough to cause a perceptible error in the determination of the fixed oil. But in dealing with very small quantities of alkaloid the estimation of the latter may, under these circumstances, be appreciably affected; cases occur in which even the whole of the alkaloid present passes into solution with the oil, and would be overlooked if attention were not paid to this property of fixed oil. On that account the petroleum-spirit solution 
must be treated as above described, and the alkaloid so isolated added to the extracts in which vegetable bases are to be looked for.

\section{EXAMINATION OF THE ETHEREAL OIL.}

\$22. Detection and Estimation.-Here, as in $\$ 11$, we will first discuss the simpler case, viz., that in which the petroleum spirit has removed ethereal, but no fixed oil, or at least only a very small quantity.

Like fixed oil, ethereal oil may also be frequently recognised under the microscope as highly refracting globules, or drops of irregular shape, which are soluble in cold alcohol (fixed oil dissolves usually in warm spirit only, if indeed it is soluble at all) and insoluble in water. Some of them yield even under the microscope several of the characteristic colour-reactions described in $\$ 142$.

We have now to estimate the amount of ethereal oil present as accurately as possible, without using any very large quantity of material. From experiments made by Osse ${ }^{1}$ the following method would appear to be the best. A quantity of the petroleum-spirit solution is accurately measured on to a carefully tared glass dish, which can be closed air-tight. (Cf. § 9.) If $5 \mathrm{cc}$. of the solution correspond to 1 gram of substance, 1 to $2 \mathrm{cc}$. will be found to be sufficient. The glass dish containing the petroleumspirit solution is then placed under a tubulated glass bell-jar

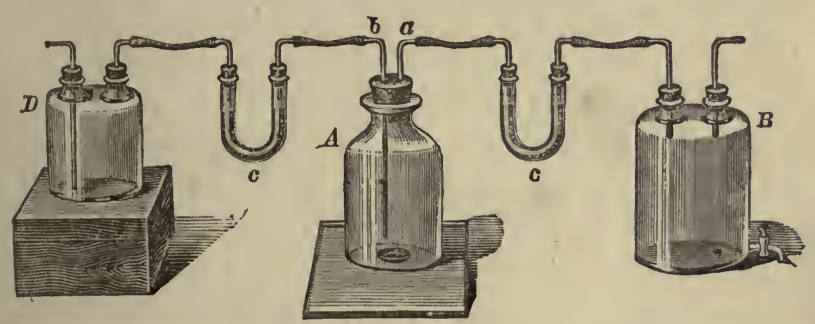

Fig. 1.

(Fig. 1), A, with ground edges resting on a ground-glass plate. Two glass tubes are then introduced through the tubulure; one of them (b) reaches nearly to the surface of the liquid to be evaporated, the other $(a)$ is cut off close below the cork, and connected with an aspirator $(B)$, so that a current of dry air may be

${ }^{1} \Lambda$ rchiv d. Pharm. [3], vii. 104 (1875) (Year-book Pharm. 1876, š62). 
drawn through the apparatus, entering by the tube $b$, and passing out through $a$. A chloride of calcium tube $(c)$ is placed before $b$, and another between $a$ and $B$, the former to dry the air entering the apparatus, the letter to prevent moist air from the aspirator passing into $A$. These precautions are necessary, for if the atmosphere in which the evaporation of the petroleum spirit is to take place be not completely dried, moisture may be deposited on the glass dish containing the solution, in consequence of the cold produced by evaporation, thus causing, of course, an increase of weight. It is advisable, therefore, to connect the first chloride of calcium tube $(c)$ with a Wolff's bottle one-third full of concentrated sulphuric acid. A current of air is then passed through the apparatus and the petroleum spirit allowed to. evaporate at the ordinary temperature until the operation appears complete; that is, until the residue has only a slight smell of petroleum spirit. The glass dish is then closed and weighed. After the weight has been accurately taken, it is again. opened, exposed for one minute to the air, closed and again weighed, and the alternate exposure and weighing repeated until the same loss in weight is observed twice in succession. This loss is then assumed to be the amount of ethereal oil that diffuses into the air per minute. It is also assumed that during every previous exposure of one minute the same weight of ethereal oil has evaporated, so that to the quantity of oil as found by the last weighing there has to be added the 'co-efficient of evaporation,' multiplied by the number of minutes the dish has been exposed. (Compare the examples of estimation in $\S 136$.) If the coefficient of evaporation is less than one milligram this correction may be omitted. Perhaps it would be advantageous to pass a current of carbonic acid through the apparatus during the evaporation of the petroleum spirit, as many ethereal oils diffuse much more slowly into that gas than into atmospheric air.

§ 23. In Presence of Fat and Resin.-After thus determining the weight of the substances dissolved in a known quantity of petroleum spirit, it must be ascertained whether the residue after evaporation is completely volatile at $110^{\circ}$, or leaves a non-volatile residue of resinous or fatty matter. In the latter case the weight must be determined and deducted from that obtained in $\$ 22$. (See $\S 138$.) If the non-volatile part constitutes the majority of the dissolved substances it may be ascertained, after the removal 
of the ethereal oil by evaporation, whether the residue is now still completely soluble in petroleum spirit. Resins which, in a state of purity, are not dissolved by petroleum spirit, may be taken into solution by means of ethereal oil, just as fixed oil carries with it alkaloids and chlorophyll; they are left undissolved on again treating with petroleum spirit the residue freed from ethereal oil. After having removed by this solvent the fixed oil, etc., that has been simultaneously extracted, the resin may be weighed alone (§ 146).

Of course it is advisable to repeat the experiments described in $\$ \S 22,23$ several times, and take the mean of the results. I need scarcely say that this method of determining the total ethereal oil does not guarantee any absolute accuracy, but as it is the only one we have at our disposition it might, for the time at least, be deserving of some notice. With less volatile oils, cinnamon, clove, etc., it has yielded very satisfactory results, but less so with terpenes, such as oil of lemon and turpentine.

$\S 24$. Distillation of Larger Quantities of Oil.-If a further insight into the composition of the ethereal oil is desired, a larger quantity must be prepared from 5 to 100 kilograms of material. For this purpose distillation in a current of superheated steam is to be recommended, the material having been if necessary previously comminuted and soaked in water. In order that the steam may thoroughly penetrate it the apparatus should be packed with alternate layers of material and straw. A distillate consisting of essential oil and water will be obtained which may be separated from one another in burettes or Florentine flasks. It should not, however, be forgotten that many ethereal oils are tolerably easily soluble in water, and a small quantity of petroleum spirit of low boiling-point should therefore be shaken with successive portions of the aqueous distillate. The petroleum spirit is allowed to evaporate in a current of carbonic acid in the apparatus described in $\S 22$, and the residue added to the oil separated from the distillate $(\S 137)$.

§ 25. Examination of Aqueous Distillate.-After separation of the oil, the action of the watery liquid on litmus should be tested. It will be frequently found to possess a distinct acid reaction, and contain formic, acetic, or other volatile acids of the fat-acid series. In such a case the higher acids in the series may be removed by shaking with ether or petroleum spirit. To 
obtain all, including those standing lower in the series, the aqueous distillate may be saturated with soda, concentrated and acidified with sulphuric acid $(1 \cdot 5)$. If an oily acid separates from the aqueous liquid, angelic or valerianic acid, or an acid higher in the series, may be looked for. (The test of smell, boiling-point, etc., may be applied, and the ultimate analysis made.) Further information on this point may be found in $§ 139,140$. If the acid liberated is soluble in water, an attempt to separate it by the addition of chloride of calcium may meet with success. Should that not be the case, formic and acetic acids may finally be tested for (behaviour to mercuric chloride, ferric chloride and nitrate of silver, the latter also reduced by acrylic acid) as well as salicylous acid. The last-named acid strikes a violet colour with ferric chloride. (See also §33.) Salicylous acid may likewise be separated from its aqueous solution by shaking with ether. For hydrocyanic acịd see $\S 34$.

Toxicodendric acid, to which Maisch partly attributes the poisonous properties of Rhus toxicodendron, appears to possess great similarity with formic, acetic, and acrylic acid. It may be isolated by distillation, and like formic acid reduces nitrate of silver and chloride of gold slowly in the cold, quickly on warming. But it does not reduce mercurous nitrate or chromic acid as formic acid does, nor does it yield the iron reaction characteristic of acetic acid, etc.; the mercuric salt dissolves with difficulty in water ${ }^{1}$ (formic acid reduces mercuric to mercurous chloride).

§ 26. Salicylic, Benzoic Acid, etc.-It must also be borne in mind that some of the acids of the aromatic series, such as salicylic and benzoic acid (\$55), are volatile with the vapour of water at temperatures as low as $100^{\circ}$, and may therefore be carried over with the steam in distilling the ethereal oil. On shaking the distillate with petroleum spirit small quantities of salicylic acid are removed, but ether and chloroform may be more advantageously employed; the latter liquid is also adapted for the isolation o benzoic acid. On evaporating the ethereal or chloroformic (or petroleum spirit) solution, both benzoic and salicylic acid are obtained as crystalline residues difficultly soluble in cold water (salicy lic acid about 1 in 300). ${ }^{2}$ The two acids may be dis-

1 Conf. Amer. Journal of Pharmacy, xxxviii. 9 (1866).

2 For particulars of the detection of salicylic acid in Viola tricolor by Mandelin in my lab oratory, see Sitzungsber. d. Dorpater Naturf. Gesellsch. Jg. 1879, p. 77, and Diss. Dorpat., 1881 ; also Pharm. Journ. and Trans. [3] xii. 627. 
tinguished by their behaviour to ferric chloride, with which salicylic acid strikes the well-known violet colour. Benzoic acid may be easily sublimed between watch-glasses. Dissolved in a drop of ammonia, the excess of which is allowed to evaporate by exposure to the air, a drop of ferric chloride produces a brownish tinge.

Cinnamic acid may also be similarly distilled over, and separated from the distillate. It may be distinguished from both the foregoing acids by its behaviour to oxidizing agents such as permanganate of potassium, with which an aqueous solution yields on warming oil of bitter almonds, whereas benzoic acid yields the same product when acted upon by a reducing agent such as sodium-amalgam. (See also § 38.)

Any cinnamic acid present might in certain cases have been produced from ethereal salts, such as, for example, styracin (cinnamate of cinnamyl), or cinnameïn (cinnamate of benzyl). Both of these compounds are soluble in petroleum spirit, and are resolved, by decomposition with an alkali, into cinnamic acid and the respective alcohol. Styracin crystallizes in needles, which, according to Scharling, ${ }^{1}$ melt at $44^{\circ}$. Cinnameïn is liquid at the ordinary temperature. The former has an odour resembling vanilla, the latter a faint smell of balsam of Peru.

If one of the three acids mentioned has been isolated, special care should be taken to ascertain whether the corresponding aldehyde is also present in the aqueous liquid, viz., salicylic, benzoic (oil of bitter almonds), or cinnamic aldehyde, and whether the acid has not been produced from the aldehyde by absorption of oxygen during or after distillation ( $\$ 33)$.

$\S 27$. Physical Properties.-The principal part of the oil obtained by distillation should be completely freed from moisture, filtered and tested with regard to its consistence. If, on standing some time in a freezing mixture, a crystalline constituent be deposited it should be separated and examined by itself. The action of the oil on polarized light should be observed ( $\$ 141)$, and fluorescence looked for ; if present, it should be ascertained whether warm water will remove a substance fluorescent either alone or on

${ }^{1}$ Annalen d. Chemie u. Pharm. Ixviii. 168. See also Rügheimer Dissertation, Tübingen, 1873 ; Kraut, Annalen der Chemie u. Pharm. clii. 129 (1869); (Amer. Journ. Pharm. xlii. 236); and Von Müller, Ber. d. d. chem. Ges. Jg. 1876, 274. 
the addition of caustic potash. Any resin that may have been obtained in the quantitative estimation of the oil ( $\$ 23)$, should be tested for a fluorescent substance by treating with distilled, or, if necessary, alkaline water ; umbelliferone should be specially borne in mind ( $\$ 43)$. The resinous constituents, which will be subsequently isolated according to $\S 36$, et seq., may be examined for umbelliferone by mixing with sand and submitting to destructive distillation, or by heating in sealed tubes with alcoholic solution of hydrochloric acid.

Further, the specific gravity of ethereal oils should.be taken. Westphal's specific-gravity balance may be advantageously employed for this purpose, especially if the quantity of oil at disposal is rather small. (Cf. $§ 141$.

It should also be ascertained what percentage of pure alcohol a spirit must contain to be miscible with the oil in all proportions. A drop only of spirit is first added to the same quantity of oil, and if the resulting mixture is perfectly clear, note should be taken whether the further addition of spirit cause a cloudiness or not. It is, however, only with freshly-prepared oil that such reactions can be considered as characteristic of the oil. Many oils undergo a change on keeping for any length of time, becoming more or less soluble in alcohol, or forming clear mixtures with small proportions, but cloudy with larger ( $\$ 141)$.

$\S 28$. Peactions. - It is, moreover, desirable to make qualitative experiments with small quantities of the ethereal oil, in order to become acquainted with their behaviour to some few re-agents. For this purpose I have recommended sulphuric acid, alone or applied in combination with sugar, nitre, or ferric chloride; nitric acid, alcoholic hydrochloric acid, solution of bromine in chloroform, picric acid, etc.

For the results which I myself, and some of my pupils, have obtained with the more important ethereal oils, see $\$ 142$.

§ 29. Detection of Sulphur.-Some ethereal oils contain sulphur, which may be detected by mixing a few drops of the oil with carbonate of soda and nitre, and introducing it into a piece of combustion tubing, about $15 \mathrm{ctm}$. long, sealed at one end. The upper part of the tube is then charged with a similar mixture of soda and nitre, and the whole ignited as if it were an ultimate analysis in miniature. About one-third of the mass from the bottom of the tube upwards is then dissolved in a little water, 
heated with excess of hydrochloric acid as long as nitrous fumes are evolved, and then tested for sulphuric acid by chloride of barium.

Warming a small quantity of an ethereal oil containing sulphur with a solution of caustic potash of specific gravity $1 \cdot 3$, and adding nitro-prusside of sodium, after diluting with water, often suffices to show the presence of sulphur by the sulphide of potassium formed striking a bluish-violet colour with the nitro-prusside.

Some ethereal oils contain nitrogen, and many of these are regarded as nitriles. This element may be detected by heating a drop of the oil with metallic sodium, dissolving the cooled mass in water, adding a drop of solution of ferric and ferrous salt, and, after a few minutes, acidifying with hydrochloric acid, when a precipitate of Prussian blue makes its appearance if nitrogen is present.

If the ethereal oil contains a sulphocyanide (oil of mustard or horse-radish), both the sulphur and nitrogen test must yield a positive result.

§ 30. Constituents. - Ethereal oils distilled from vegetable substances are generally mixtures that can be separated into their constituents. If this is to be attempted we must, from the first, admit that, in the present state of our knowledge, an exact quantitative separation is not to be thought of. The principal reason for this must be sought for in the ease with which ethereal oils undergo decomposition, and the great disposition many of them show to form polymers. In the majority of cases only one method of separating the constituents of an oil is feasible, viz., that of fructional distillation, which must be repeated until products of constant boiling-point have been obtained. But it is in this very distillation that a change in the oil often takes place, either by the formation of polymers of the original oil with higher boiling-points, or by the production of hydrocarbons by the liberation of the elements of water from constituents of the oil containing oxygen.

An important improvement in these operations might perhaps be made in conducting the distillations under diminished pressure. In order to make this modification available, the temperature must first be ascertained at which the more commonly occurring constituents of oils can be distilled. Many of the terpenes present in ethereal oils may be distilled under the ordinary pressure at $155^{\circ}$ to $157^{\circ}$; many of their polymers at about $190^{\circ}$; others at 
about $250^{\circ}$. This knowledge forms, of course, a good basis on which a separation may be attempted.

For these and other fractional distillations which may have to be performed in the analysis of plants, small flasks provided with the dephlegmators recommended by Linneman may be used. (Cf. § 143.)

$\S 31$. Stearoptenes, etc.-The following are the more important constituents of ethereal oils that have up to the present time been observed : Terpenes of the composition $\mathrm{C}_{10} \mathrm{H}_{16}$ often boiling at $155^{\circ}$ to $157^{\circ}$; polymers of the same, of the formula $\mathrm{C}_{15} \mathrm{H}_{24}$ and $\mathrm{C}_{20} \mathrm{H}_{32}$, boiling frequently at about $190^{\circ}$ or about $250^{\circ}$; oxygenated compounds of the formula $\mathrm{C}_{10} \mathrm{H}_{20} \mathrm{O}, \mathrm{C}_{10} \mathrm{H}_{18} \mathrm{O}, \mathrm{C}_{10} \mathrm{H}_{10} \mathrm{O}$, $\mathrm{C}_{10} \mathrm{H}_{14} \mathrm{O}, \mathrm{C}_{10} \mathrm{H}_{12} \mathrm{O}, \mathrm{C}_{10} \mathrm{H}_{12} \mathrm{O}_{2}$; hydrocarbons of the formula $\mathrm{C}_{10} \mathrm{H}_{14}$ are more rarely to be found; still less frequently those of the $\mathrm{C}_{\mathrm{n}} \mathrm{H}_{2^{\mathrm{n}}}$ series. Of these constituents of oils, it is noticeable that those containing oxygen crystallize in the cold more readily than hydrocarbons of the formula $\mathrm{C}_{10} \mathrm{H}_{16}$, and to the former, therefore, our attention must be specially directed in the examination of the crystalline 'stearoptenes' obtained by cooling the oils (with the exception of otto of roses $=\mathrm{C}_{\mathrm{n}} \mathrm{H}_{2 \mathrm{n}}$ ).

If such a stearoptene has been isolated, its purification should be attempted by repeatedly crystallizing from alcohol or ether, pressing the crystals each time between blotting-paper. The coefficient of refraction may then be ascertained in the alcoholic solution of the pure substance; the melting-point, boiling-point, and vapour-density determined; and, finally, an ultimate analysis made. It should also be ascertained whether hydrocarbons can be obtained by distillation over phosphoric anhydride or chloride of zinc.

The liquid portions of the various fractions should be subjected to similar experiments, with the exception of the last. It will frequently be found that ethereal oils containing oxygen, as well as those containing hydrocarbons, of the formula $\mathrm{C}_{15} \mathrm{H}_{24}$ and $\mathrm{C}_{20} \mathrm{H}_{32}$, yield very characteristic colour reactions with the reagents detailed in $\$ 28,142$; whilst oils consisting principally of terpenes of the formula $\mathrm{C}_{10} \mathrm{H}_{16}$ show less inclination to give marked reactions. These latter oils may often be purified for ultimate analysis by distillation over metallic sodium.

$\S 32$. Other Constituents.-Besides the constituents already mentioned-which indeed, although frequently agreeing in their 
composition, have, when prepared from different plants, somewhat dissimilar properties (odour, behaviour to polarized light, etc.) some ethereal oils contain other substances which may belong to tolerably distant groups. Aldehydes, ethereal salts, alcohols, acids, etc., have been found in various oils.

$\S 33$. Aldehydes.-If an aldehyde is to be looked for in an ethereal oil, it must first be ascertained whether that oil precipitates metallic silver from an ammoniacal solution of the nitrate. ${ }^{1}$ If this is the case, it must be shaken with a concentrated solution of acid sulphite of soda. The majority of aldehydes are dissolved by acid sulphite of soda, and may be separated from other constituents which do not enter into such combination by removing the aqueous liquil. The aldehyde may then be liberated from combination by neutralizing with caustic soda or decomposing with dilute sulphuric acid, and, when thus separated, should be tested as to its physical properties, odour, etc. It should also be ascertained if it produces a crystalline precipitate in ethereal solution of ammonia. Finally, an ultimate analysis may be made.

Of the aldehydes to which particular attention should be directed, I may mention those of pelargonic, capric and methylcapric acid, of angelic, cinnamic, salicylic, and benzoic acid (\$\$ 25, 26). Many, perhaps all, vegetable substances containing chlorophyll, when distilled in the fresh state, appear to yield a substance with the characters of an aldehyde. ${ }^{2}$

$\S 34$. Volatile Acids.-Acids may be removed from the ethereal oil by shaking with dilute solution of potash or soda, and may be liberated, after evaporation of the solution, by the addition of dilute sulphuric acid. (Cf. $\S \S 25,139$.$) Besides the volatile$ acids already mentioned, the possible presence of hydrocyanic acid, which is partially converted into formic acid by shaking with soda, is not to be forgotten. It may best be looked for in the aqueous part of the distillate ( $(25)$, and recognised by the well-known silver precipitate and sulphocyanide and Prussianblue tests.

$\S 35$. Ethereal Salts.-If an essential oil is to be examined for ethereal salts that may be mixed with it, it should be remembered that such salts may be decomposed by heating in autoclaves with

1 See Tollens, Ber. d. d. chem. Ges. xv. 1635 ; Salkowski, ibid, 1739 (1882).

2 See Ber. d. d. chem. Ges. xiv. 2144, 2508, for an account of this most interesting observation. 
solution of caustic soda or with baryta-water, yielding a salt of the acid, and the alcohol corresponding to the basic radical contained in them. This latter body may be separated by distillation. ${ }^{1}$ Acetate of octyl, which occurs in the oil of Heracleum, would thus yield an acetate and octyl alcohol. Certain substitution acids, such as methyl-salicylic acid, might be similarly decomposed; this, for instance, would yield a salicylate and methylic alcohol. The latter class of compounds would split up on treatment with hydriodic acid; methyl-salicylic acid would thus yield iodide of methyl and salicylic acid.

If the alcohols and iodides thus liberated are tolerably freely soluble in water, and therefore not mechanically separable, they must be removed by fractional distillation, in which chloride of calcium and other hygroscopic substances may be often used with success. $^{2}$ Their identification should rest upon the determination of boiling-point and vapour-density, and the ultimate analysis. The same applies in the case of an ethereal oil containing an alcohol a priori.

Of the alcohols that may be more commonly separated from ethereal oils, methyl alcohol boils at $58.6^{\circ}$, ethyl at $78.4^{\circ}$, propyl at $96^{\circ}$, isopropyl at $83^{\circ}$ to $84^{\circ}$, butyl at $116^{\circ}$, isobutyl at $109^{\circ}$, amyl at $130^{\circ}$, pseudo-amyl at $120^{\circ}$, hexyl at $157^{\circ}$, heptyl $175.5^{\circ}$ to $177 \cdot 5^{\circ}$, octyl at $196^{\circ}$ to $197^{\circ}$.

To distinguish between a primary, secondary, and tertiary alcohol, V. Meyer and Locher recommend conversion into iodide. This is mixed with twice its weight of nitrate of silver and a little sand, and distilled, the distillate shaken with strong solution of caustic potash and nitrite of potassium, and then acidified with dilute sulphuric acid. If a primary alcohol is present the mixture will turn red, if a secondary, blue (which may be removed by shaking with chloroform), whilst tertiary alcohols give colourless products of decomposition. In the series of secondary alcohols the reaction succeeds as far as amyl alcohol, in the series of primary alcohols as far as octyl alcohol (Gutknecht). ${ }^{3}$

The acids separated from the ethereal salts, obtained by decomposing the alkali or barium salt with sulphuric or phosphoric acid, may be examined according to the directions given in $\$ \$ 25,34,130$.

${ }^{1}$ Cf. Wanklyn, Chem. News. xxvi. 134.

2 If the ethereal salt yield ethylic alcohol as a product of decomposition, the amount may be directly estimated from the specific gravity of the distillate.

3 See also Heil and Urech, Ber. d. d. chem. Ges. xv. 1249 (1882). 
III.

\section{Examination of the Substances Soluble in Ether.}

\section{RESINS AND THEIR ALLIES.}

\$36. Extraction.-After the examination of the substances dissolved in petroleum-spirit has been carried as far as possible, the residue (cf. $\S 9$ ), thoroughly washed with the menstruum, should be removed from the filter (which is to be kept) dried at the ordinary temperature, and then macerated for seven to eight. days with pure ether. It is advisable to use the same vessel that has been employed for the treatment with petroleum spirit. If it has been well washed there is no necessity for being minutely particular to bring the whole of the residue on to the filter. The same vessel should, if possible, be reserved for the extraction with alcohol, to be described in $\$ 47$, and the filtration effected through the same filter that has already done duty for the petroleum spirit and ether extracts. I allow the ether destined for this purpose to stand for several weeks over porous chloride of calcium, and then rectify it after carefully separating the calcium salt. To obtain constant results in such analysis it is necessary to have the ether as free as possible from water and alcohol. Ordinary commercial ether would, for instance, extract a portion of the tannin (sometimes more, sometimes less) from parts of plants containing that substance, whilst ether, purified as described, does not usually produce this effect. As it is not well possible to remove the whole of the tannin with commercial ether, I prefer to refrain from extracting any of it with that menstruum, and remove the whole subsequently with alcohol. To attain this end I avoid the employment of a high temperature in extracting with ether. Indeed, I am of opinion that in the course of the analysis of plants, it is better in the majority of cases to 
allow the solvent to act at the ordinary temperature. Some instances of special estimations may be excepted in which separate portions of the material may be extracted warm.

After allowing the maceration with ether to proceed for about eight days, the first estimation to be made is that of the total substances dissolved, which may be effected by evaporating an aliquot part, or the whole of the extract, in a flat-bottomed glass dish. I usually employ a measured quantity of ether, say 5 to $10 \mathrm{cc}$., for every gram of substance under examination, macerate in a well-closed flask, and replace any ether that may have been lost by evaporation during the process. After well shaking I take a certain number of cc. of the clear or (cf. $\S 9$ ) filtered liquid for evaporation. The residue must be dried at $100^{\circ}$ to $110^{\circ}$, till the weight is constant, and this then noted. It should be ascertained whether any fatty matter which has escaped extraction with petroleum spirit is mixed with the residue, and if this is the case it should, if possible, be removed by washing with the latter liquid, its weight noted, added to the amount found in $\S 9$, and subtracted from the substances dissolved by ether. It must also be borne in mind that all fats are not necessarily soluble in petroleum spirit. It is well-known that castor oil forms clear mixtures with certain, but not all proportions of that solvent. ${ }^{1}$

The remainder of the ethereal extract is filtered from the residual powder, the latter washed, and extract and washings allowed to evaporate at the ordinary temperature. The residue of the substance is freed from ether at the same temperature as speedily as possible.

$\S 37$. Chlorophyll.-The ethereal extract may also be tested before evaporation for chlorophyll, as described in $\$ \$ 20,132$, et seq. I have already observed that this substance is more easily and completely removed by ether than by petroleum spirit.

$\S 38$. Portion Soluble in Water.-That part of the ethereal extract which has been evaporated at the ordinary temperature may, if possible, be powdered or brought into as fine a state of division as practicable by triturating with washed sand or pure siliceous earth (Kieselguhr), and treated with cold water. In the aqueous solution substances soluble in water, such as hocmatoxylin, gallic acid, catechin, pyrocatechin, salicylic acid, benzoic acid,

1 Jahrb. f. Pharm. 1876, p. 369 (Year-book Pharm. 1876, p. 356). 
salicin and other glucosides, and alkaloids may be looked for. The latter, however, may, as a rule, be more easily extracted with water containing acetic or sulphuric acid. A measured portion of the aqueous liquid may be evaporated, and the residue weighed. For the detection of hæmatoxylin and allied substances see $§ 150$; of gallic acid, etc., $\S 151$; of salicylic and benzoic acid, $\S \S 26,34$; of glucosides, $\$ \S 54$ et seq., 165 et seq.; of alkaloids especially, $§ 63$ .et seq., 171 et seq.

§ 39. Portion Soluble in Alcohol.- The part insoluble in water should be again dried and extracted in a similar manner with absolute alcohol. If the plants under examination contain much resin it will often be observed that a part only of the resinous constituents, etc., dissolves in alcohol. The amount of matter soluble in alcohol, as well as in ether, must then be determined by evaporating the alcoholic solution and weighing the residue.

We have thus determined $(a)$ the total substances dissolved by ether, $(b)$ any fat that may have been extracted, $(c)$ the substances soluble in ether and water, $(d)$ substances insoluble in water, soluble in ether and in alcohol, and (e) substances extracted by ether insoluble in water and alcohol.

The next step is to obtain a further insight into the nature of the resinous substances soluble in ether alone, as well as those soluble in ether and alcohol.

$\S 40$. Microchemical Examination.-The microscopical examination shows that the resins are present partly in the cell wall, saturating it as it were, and partly in the form of exudations either within or upon the cells. Special attention should be paid to their insolubility in water, solubility in alcohol or ether, to the red colour which, according to Müller, is produced with resins by alcoholic tincture of alkanna, violet or blue (Hanstein) by aniline. Some of the reactions enumerated in $\S 146$ might also be made available for microchemical analysis.

In the macrochemical examination it should first be ascertained whether the resin cannot be separated into different component parts by the use of other solvents, such as chloroform, benzene, bisulphide of carbon, acetone, acetic ether, or boiling absolute alcohol, or finally by precipitating the concentrated ethereal solution with alcohol, petroleum spirit, or other suitable liquid. Similarly, if a substance soluble in ether has not from the first been obtained in crystals, slow evaporation of the solution in the 
last-named solvents (prepared warm if necessary) should be resorted to in the attempt to crystallize the substance, or ta separate it into a crystalline and an amorphous portion.

If the endeavour,to obtain crystals be successful, the crystalline form should if possible be determined, and care should be taken to observe whether different crystalline forms can be distinguished. under the microscope, rendering it probable that the substance. under examination is a mixture. ${ }^{1}$

§ 41. Behaviour of Resins to Reagents.-It will further be of special interest to learn whether the substances soluble in ether, insoluble in alcohol and water, are dissolved by alcoholic or aqueous solution of caustic potash, in which case there would be reason to suspect the presence of an acid resin ( $\$ 145)$. If insoluble in these liquids it might be assumed that the body under examination is an indifferent resin, or a resin-anhydride not easily susceptible of decomposition. These and the following experiments should be conducted with larger quantities of the substance soluble in ether, specially prepared for this purpose.

If an indifferent resin or stable resin-anhydride were present it might first be purified by recrystallization or reprecipitation, etc., and then an ultimate analysis made. It should be tested for colour-reactions with concentrated sulphuric acid alone and in conjunction with sugar. If ethereal solution of bromine yield a substitution product, its composition should be ascertained. It should also be noticed whether the resin-anhydride is easily oxidized and dissolved by nitric acid, or whether that takes place. only with difficulty; whether water precipitates the unchanged resin after the action of the acid, or whether oxidation products are formed ; and if so, what is their nature, as, for instance, picric ${ }^{2}$ or oxalic acid (\$§81, 219), succinic acid (§220).

$\S 42$. Action of Fused Potash.-It is further important to become acquainted with the products formed under the influence of fused caustic potash or soda. ${ }^{3}$ The finely powdered substance, in quan-

${ }^{1}$ For particulars of a case of this kind, viz. the separation of a mixture of resins obtained from larch-fungus, see Masing, Pharm. Zeitschr. f. Russland, Jg. 9, p. 394 (1870).

2 Bitter yellow crystals belonging to the rhombic system sparingly soluble in cold water, more freely in boiling, soluble in alcohol and ether. It stains skin and wool yellow, and yields a blood-red liquid when an alkaline solution is: warmed with cyanide of potassium, sulphide of potassium, or grape sugar.

${ }^{3}$ Cf. Hlasiwetz and Barth, Annalen der Chemie und Pharm. cxxxiv. 265; exxxviii. 61 ; cxxxix. 77. 
tities of not much more than 10 grams at one operation, is mixed with 6 to 8 parts of caustic alkali, and introduced in successive portions into a previously heated silver crucible, and the heat continued, stirring occasionally with a silver spatula until the mass is in a uniform state of fusion. After cooling the contents of the crucible are dissolved in water, and a slight excess of sulphuric or hydrochloric acid added. The decomposition products, which are specially to be looked for, are butyric and valerianic acid (cf. $\S \S 25,34,139$ ), pyrogallol, phloroglucin, and resorcin, benzoic ( $(26)$, paraoxybenzoic, and protocatechuic acid. The majority of these substances may be removed by ether after acidifying. Volatile fatty acids might be previously extracted by shaking with petroleum spirit.

Resorcin.-After the fatty acids have been removed by petroleum spirit, resorcin may be extracted from the aqueous liquid by shaking with ether and distilling the ethereal solution after separation. It forms crystals melting at $99^{\circ}$, has a sweetish taste, and strikes a dark violet colour with solution of ferric chloride, violet with chloride of lime, and rose-red with ammonia. It reduces ammoniacal solution of nitrate of silver.

Phloroglucin is also very sweet-tasted, and resembles resorcin in many of its reactions, but is coloured reddish-violet with ferric chloride, and transient reddish-yellow with solution of chlorinated lime. The anhydrous crystals melt at $220^{\circ}$.

Pyrogallol tastes bitter, is soluble in water, alcohol, and ether, melts at $115^{\circ}$, reduces ferric to ferrous salts, colours the latter blue-black, and separates gold, silver, platinum and mercury from solutions of their salts. An alkaline solution exposed to the air rapidly assumes first a red, then a brown colour; with lime-water it passes through a transient violet and purplish-red tint.

Protocatechuic Acid has an acid reaction, is sparingly soluble in water, strikes no colour with pure ferrous, but yields a dark-green solution with pure ferric salts. With mixtures of both ferric and ferrous salts a violet tint is produced. The green liquid obtained by the action of ferric chloride is turned red by potash, and then assumes a violet tint on addition of hydrochloric acid. It reduces the metal from an ammoniacal silver solution, but is distinguished from the three foregoing substances by not reducing alkaline tartrate of copper. With acetate of lead it yields a precipitate soluble in acetic acid. 
Paraoxybenzoic Acid melts at $210^{\circ}$, dissolves with difficulty in cold water, and gives, with ferric chloride, a yellow precipitate easily soluble in excess.

For Orcin and Betaorcin see $\$ 158 .^{1}$

$\S 43$. Dry Distillation of Resins.-It has already been mentioned in $\S 27$ that the dry distillation of part of the resin may lead to useful results. Besides the umbelliferone mentioned in that section, pyrocatechin ( $\$ 151)$, pyrogallol, etc., should be borne in mind. The first of these substances is fluorescent, and dissolves in boiling water, alcohol, and ether; the second strikes a green colour with solutions of ferroso-ferric salts.

$\S 44$. Examination of Portion Soluble in Alcohol.-The remainder of the mixture of resins extracted by ether-that is, the part soluble in alcohol-may be tested as directed in $\$$ - 40 to 43 . Acid resins will be found here more frequently than in the portion insoluble in alcohol. If the resin soluble in alcohol dissolves either partially or wholly in an aqueous solution of potash as well, the solution in the latter liquid may be shaken with ether to ascertain if any substance can be removed by that solvent. It was by this means that I isolated poconiofluorescin from peony-seed (\$ 147). Chrysophanic acid and allied substances ( $\S 148,149$ ) should also be tested for, as well as quercitrin, quercetin (§152), and the bodies discussed in $\$ 150$ to 158 .

$\S 45$. Acids Produced by Action of Alkalies.-Attention must further be paid to the fact that the action of caustic alkalies on certain anhydrides nearly related to the resins-as, for instance, santonin-may result in the formation of alkali salts, which are not of necessity, on the addition of excess of acetic or hydrochloric acid, instantly decomposed with reproduction of insoluble anhydride. In the case of santonin, santonic acid is liberated on acidulating the alkaline aqueous solution. Any ordinary acid resin mixed with it may be removed by precipitation with hydrochloric or acetic acid and immediate filtration. The santonin is deposited only after standing several days, but can be extracted at once by shaking with chloroform. A method that I have proposed for the estimation of santonin is based upon this fact, and will be described in $\S 154$.

\$46. Direct Extraction with Ether.-A portion of the powdered material may, without further treatment, be extracted with ether,

${ }^{1}$ For ferulic acid compare Jahrb. f. Pharmacie, 1866, p. 95. 
and the substances thus dissolved estimated. In the majority of cases the weight will be the same as the sum of the substances extracted by petroleum spirit according to $\S 9$, and ether according to $\S 36$. If there is a deficiency, the residue should be treated with petroleum spirit, in which case attention would be directed to a substance other than an ethereal or fixed oil. The residue after exhaustion with ether, and, if necessary, petroleum spirit, may be dried and boiled with chloroform or bisulphide of carbon, to ascertain if such substances as caoutchouc, etc. can be extracted (§ 127). 
IV.

\section{Examination of the Substances Soluble in Absolute ALCOHOL.}

RESINS, TANNINS, BITTER PRINCIPLES, ALKALOIDS, GLUCOSES, ETC.

§ 47. Extraction.-The residue of the substance under examination after exhaustion with petroleum spirit and ether (cf. $\S 36$ ) is removed from the filter, dried at the ordinary temperature, and treated with $10 \mathrm{cc}$. of absolute alcohol for every gram of original substance. After the lapse of five to seven days the alcohol lost by volatilization is replaced, and the whole well shaken. It is then filtered through the same filter that has been used for the previous operations, any evaporation of alcohol being prevented as carefully as possible. A measured quantity of the filtrate is next evaporated in a tared platinum dish and dried until the weight noted is constant. It is then incinerated, and the ash deducted from the weight of the dry substance. After having thus estimated the total organic matter insoluble in petroleum spirit and ether, but soluble in alcohol, the residue on the filter may be washed with absolute alcohol, and the washings, with the remainder of the filtrate, concentrated. This may best be done by distilling in a flask, under diminished pressure. The liquid remaining after distillation is poured into a glass dish, and allowed to evaporate, at the ordinary temperature, over sulphuric acid.

$\S 48$. Estimation of Portion Soluble in Water:-The dry residue thus obtained is first treated with a measured quantity of water. To ascertain the amount soluble in this menstruum, as well as in alcohol, a measured quantity of the solution is similarly evaporated, dried at $110^{\circ}$, and weighed.

The remainaier of the aqueous extract is reserved for the experiments detailed in $\$ \$ 49,50,70$; that which is insoluble in 
water is treated with water containing a little ammonia ( 1 in 50) as long as anything is removed. The ammoniacal extract may be evaporated with a slight excess of acetic acid, the residue rinsed on to a filter with a little water, washed, dried, and weighed. The brownish mass thus left on the filter is, as a rule, to be regarded as phlobaphene ( $\$ 108$; see also $\$$ 160, 163), resulting from the decomposition of tannin. The portion of the aqueous extract insoluble in ammoniacal water may be again dried over sulphuric acid, and then subjected to similar treatment as the resin soluble in ether (cf. $\$ \varsigma 39$ to $45 ; 145,146$ ). If there is reason to suspest the presence of an alkaloid soluble in alcohol, but insoluble in ether, the residue, after treatment with ammoniacal water, may be digested with water containing a little sulphuric acid. (For alkaloids see $\$ 55$, et seq.; 63 , et seq.; 171, et seq.)

\section{EXAMINATION OF THE TANNIN.}

$\$ 49$. Detection. - If the aqueous solution obtained from the evaporation residue of the alcoholic extract is coloured blue-black by a ferroso-ferric salt and precipitated by gelatine, solution of acetate of lead is to be added in slight excess. The resulting precipitate is immediately collected on a tared filter, washed with water (not too long, three or four times, with 3 to 5 cc.), dried, and weighed $(\$ 52, \mathrm{I}$.). It is then removed from the filter, which is burnt in a porcelain crucible with a little nitrate of ammonia; the precipitate itself is next incinerated, and the whole finally ignited in the blow-pipe flame until the weight is constant. This is then deducted from the weight of the precipitate, and the remainder noted as tannic acid, or bitter principle precipitated by oxide of lead, or vegetable acids precipitated by lead $(\S 80)$. The filtrate from the lead precipitate is treated according to $\S 70$.

$\$ 50$. Detection continued.-The same treatment is repeated with a similar quantity of the watery extract obtained in $\S 48$, substituting acetate of copper for acetate of lead $(\S 52, \mathrm{II}$.). Here, too, the amount of oxide of copper in the precipitate is to be determined by following the same directions and deducted from the weight of the precipitate. If the estimation with the copper salt yields the same result as that with the lead, it is tolerably certain that only tannic acid has been precipitated. But if lead throws down more matter than copper we are generally justified in assuming that the former precipitates substances other than 
tannin, such as other acids, or bitter principles, the amount of which may be approximately determined by deducting the weight of the organic matter contained in the copper precipitate from that contained in the lead. Under these circumstances the weight of the organic substances precipitated by copper sometimes represents approximately the tannin contained in the material $(\$ \S 52,80)$. It must, however, be admitted that the great difference in the tannins occurring in nature prevents such a result being looked for in every case.

. $\S 51$. Reactions. - The following reactions are common to all tannins : they are precipitated from aqueous solution by gelatine, by many albuminous substances, by acetate of lead and copper, stannous chloride, etc. ; they reduce, at least when warm, alkaline solution of copper as well as solutions of gold and silver salts; they strike an inky or dark-green colour with ferroso-ferric salts and transform skin into leather. Some tannins are precipitated by mineral acids, by tartar emetic and by alkaloids, but it is frequently observable that an alkaloid and tannin which occur together in the same plant do not form an insoluble compound.

For the microscopic detection of tannin the reaction with iron salts may be made use of. Cells containing tannin are moreover coloured reddish-brown with bichromate of potash, violet-red with aniline and reddish or violet with dilute solution of chloride of zinc and iodine. (See note to $§ 249$.)

The great difference shown by the various tannins ( $\$ 159$ et seq.) makes it exceedingly difficult to give any general rules for their estimation. Some of my pupils ${ }^{1}$ have therefore at my instance tested the behaviour of the more important tannins to the reagents that have been recommended for their quantitative estimation. Before I give a short résumé of the results they have obtained I should like to observe that, in my opinion, the estimation of the tannin in the alcoholic extract, prepared as I have described, is preferable to the determination in the aqueous extract, provided of course that the material is very finely powdered, that the tannin is insoluble in ether free from alcohol, and that the alcoholic liquid has been evaporated under diminished pressure

${ }^{1}$ Compare Günther, Pharm. Zeitschr. f. Russland, Jg. 1870, pp. 161, 193, 225 , and 'Beiträge zur Kenntniss der in Sumach, Myrobalanen etc. vorkommenden Gerbsäuren,' Diss. Dorpat, 1871, and other Dorpat dissertations subsequently referred to. 
and dried as directed in $\S 47$. One advantage in employing alcohol to extract the tannin, as already recommended by Loewe, is the exclusion of the vegetable mucilage (so-called pectin) and similar substances which may under certain conditions introduce a very great error into the estimation. Another reason in favour of the use of alcohol is to be found in the fact that, if the material contains a large quantity of albuminous matter, water will frequently only partially remove the tannin, and that many tannins are much more easily decomposed by evaporation in an aqueous than in an alcoholic solution. It may happen, it is true, that cold absolute alcohol will not in some cases extract the whole of the tannin from vegetable substances that are very rich in albumen, but even in such cases I would prefer treating the residue, after extraction with ether, with boiling alcohol to exhausting it with water. (See also $\$ \S 95,162$.

Special emphasis must, however, be laid on the importance of getting rid of the whole of the alcohol by distillation, if that menstruum has been employed, as almost all the following determinations of tannin are made in aqueous solution, and the admixture of even small quantities of alcohol might cause great error.

$\S 52$. Let us now review the more important methods that have been recommended for the estimation of tannin.

I. Acetate of Lead.-Pribram ${ }^{1}$ has proposed precipitation with neutral acetate of lead. If care be taken not to introduce too great an excess of the prexcipitant, the precipitation of most tannins is tolerably complete, and it is only in the case of gallotannic acid, catechu-, kino-, and caffeo-tannic acid that part remains in solution on account of the slight solubility of the lead salt. But as the precipitates are not invariably of constant composition it is difficult to estimate the tannin by titration with lead solution. Some of the precipitates (tannic acids from oak- and willow-bark) are decomposed by prolonged washing with water, the tannic acid partly passing into solution and undergoing change. It was for these reasons that I have recommended the precipitation to be made in not over-dilute solutions, and directed that the washing should not be continued too long, and that the tannin should be deter-

${ }^{1}$ Zeitschr. f. anal. Chemie, v. 455 (1866). Compare also Jacobson, Chem. techn. Repert. 1866, ii. 85 ; Stein, Schweiz. polyt. Zeitschr. ii. 169 ; Gietl, Zeitschr. f. anal. Chemie, xi. 144 (1872); and Schmidt, Zeitschr. d. österr. Apothekervereins, xii.p. 374 (1874); (Am. Journ. Pharm. 1874, 427). 
mined from the organic matter in the dried precipitate. In this way the tannin in rhatany, tormentilla, sumach, divi-divi, myrobalans, knopper-galls, oak-bark, and willow-bark may generally be satisfactorily estimated. Gallo-tannic acid at times also yields good results.

II. Acetate of Copper has been suggested by Sackur ${ }^{1}$ as a precipitant for tannin. The composition of the precipitate is, however, seldom constant even when working with the same tannic acid; and here, too, it has proved advisable to precipitate in tolerably concentrated solutions, not to wash too long, and to estimate the tannin gravimetrically, as above described.

III. Stannous Chloride and Ammoniacal Stannous Chloride, which have been recommended by Risler-Beunat ${ }^{2}$ and Persoz, ${ }^{3}$ for the estimation of tannin, precipitate most tannic acids less completely than the two foregoing reagents. The precipitates moreover form slowly, but are in the majority of cases tolerably constant in composition. On account of the solubility of the precipitate in water, the estimation will here, too, be most accurate when the washing is not continued too long, the precipitate dried, impregnated with nitrate of ammonia, ignited, and the resulting oxide of tin weighed. The loss by ignition gives the weight of the tannin. But since the advantage in obtaining precipitates of constant composition cannot compensate for the deficiencies of the method already mentioned, I have not further thought of employing the precipitation with stannous chloride for the purposes we have now in view.

IV. Tartar Emetic, which has been recommended by Gerland ${ }^{4}$ and $\mathrm{Koller}^{5}$ for the volumetric estimation of tannin, will yield

${ }^{1}$ Gerberzeitung, xxxi. 32. See also Wolff, Krit. Blätter f. Forst und Jagdwissensch. xliv. 167 ; Fleck, Wagner's Jahresber. f. techn. Chem. Jg. 1860 , p. 531 ; Hallwachs, Zeitschr. f. anal. Chem. v. 234 (1866).

2 Zeitschr. f. anal. Chem. ii. 287 (1863).

3 Traité de l'Impression des Tissus, i. 282. The results obtained by the method recommended by Persoz, in which the amount of tannin is calculated from the volume of the precipitate, are, according to Gauhe (Zeitschr. f. anal. Chem. iii. 130, 1864) and Cech (Stud. über quant. Best. der Gerbsäuren, Inaugural Dissertation, Heidelberg, 1867), too high. I avail myself of this opportunity to draw attention to the works of the two last-named authors, which are intended as a critical review of the more important methods of estimating tannic acid. (See Procter, Pharm. Journ. Trans. [3], vii. 1020; Allen, Commercial Organic Analysis, London, 1879.)

${ }^{4}$ N. Jahrb. f. Pharm. xxvi. 20 (1866); (Amer. Journ. Pharm. xxxv. 519).

${ }^{5}$ Koller employed this method in estimating the tannic acid in orange-peel (N. Jahrb. f. Pharm. xxv, 206, 1866). 
satisfactory results in some few cases only, because, even if the solution be mixed with chloride of ammonium, it is difficult to ascertain when a sufficient quantity of the reagent has been added, and because some of the tannin precipitates so produced are rapidly decomposed. Some tannins (rheo-tannic acid) are not precipitated at all by tartar emetic.

V. Ammoniacal Solution of Acetate of Zinc. - This reagent should, according to Terreil, ${ }^{1}$ Carpené, ${ }^{2}$ and Barbieri, ${ }^{3}$ be used in the following manner for the estimation of tannic acid. The liquid to be precipitated is brought to the boiling-point, an excess of the zinc solution added, and, after concentration by evaporation, the mixture is cooled and filtered. The precipitate is then dissolved in sulphuric acid, and the tannin estimated by titration with permanganate of potassium. I must admit that some tannic acids may be determined in this manner, but I must also draw attention to the fact that all tannins occurring in vegetable substances do not exercise the same influence on permanganate of potassium; that is, one tannic acid may differ from another in the amount of permanganate a given quantity can decolourize, and this value of the tannin in terms of permanganate must in many cases be first determined. It is partly on this account that the estimations of the tannin in wine, made according to this method, are of but little value.

VI. Ferric Acetate, in conjunction with acetate of soda, has been used by Handtke ${ }^{4}$ for the estimation of tannic acid in oak-bark, valona, divi-divi, sumach and catechu. He found the reagent unsuited for the precipitation of the tannin present in Rheum, various species of Filex, coffee and other plants ; and even with the firstnamed substances it was only when the concentration was such that the precipitate contained 45.8 per cent. of oxide of iron that the estimation yielded satisfactory results.

Still less feasible is Wildenstein's ${ }^{5}$ colorimetric examination, which is based upon the intensity of the colour produced by the solution on paper impregnated with ferric citrate.

VII. Titration with Permanganate of Potassium..-Monier, ${ }^{6} \mathrm{Cech},{ }^{7}$

1 Zeitschr. f. anal. Chem. xiii. 243 (1874).

2 Ibid. xv. 112 (1876).

3 Ibid. xvi. 123 (1877). See also Kathreiner, ibid. xviii. 113 (1879).

4 Journ. f. pr. Chem. Ixxxi. 345.

5 Zeitschr. f. anal. Chemie, ii. 137 (1863).

${ }^{6}$ Compt. rend. xlvi. 447.

7 Loc. cit. 
Löwenthal, ${ }^{1}$ and others, have shown that the tannin contained in many vegetable substances may be estimated with sufficient accuracy for technical purposes by titrating with solution of permanganate of potassium. In dealing with vegetable infusions, however, almost all authors agree that if a satisfactory result is to be obtained the solution to be titrated must be very dilute (about 1 in 400), and the oxidation incomplete. Löwenthal and others have found the following to be the most advantageous method of procedure. The liquid under examination is mixed with a measured quantity of solution of indigo-carmine, the value of which, in terms of permanganate, has been previously determined. The permanganate solution is then run in till the blue colour changes to green. The value of the pure tannin in terms of the reagent must have been previously determined by experiments with weighed quantities of the same. By such experiments Gïnther ascertained that 16 parts of oxygen from the permanganate oxidized 32.5 parts of gallo-tannic acid, 33.0 of sumach-tannic acid, $25.0(5.54)$ of catechu-tannic acid, $24.0(5.32)$ of catechuic acid, 228.0 of kinotannic acid, 34 to 37 of rhatania-tannic acid, 35 of tormentillatannic acid, 34 of caffeo-tannic acid, and 32 of oak-bark-tannic acid.

Neugebauer ${ }^{3}$ estimated the tannic acid in oak-barks with permanganate by taking advantage of the power possessed by animal charcoal of absorbing tannic acid, and thus removing it completely from its aqueous solution. He divided the infusion to be examined in two equal parts. The one was titrated direct with permanganate, the other after the absorption of the tannic acid by animal charcoal. The amount of tannin present was then calculated from the difference, the assumption being made that the substances which acted upon permanganate in the liquid after treatment with animal charcoal were foreign bodies. Löwenthal (see below) titrates a part of the tannin solution direct, another part after precipitation with solution of gelatine(XII.). From the difference in the quantity of permanganate used the tannin is calculated.

1 Journ. f. pr. Chem. Ixxxi. 150.

2 Owing to a mistake in the calculations, the figures here given for catechutannic acid and catechuic acid are much too high. The correct numbers are placed in brackets after them. Lehmann, in checking the experiments (Vergl. Unters. einiger Catechu- und Gambier-Proben. Diss. Dorpat, 1880), found that 16.0 parts of oxygen were equivalent to $5 \cdot 14$ parts of catechu-tannic acid and 4.84 catechin.

3 Zeitschr. f. anal. Chem. x. 1 (1871). 
If a solution contain both gallic and tannic acid, or catechin and catechu-tannic acid, both may be approximately estimated by Löwenthal's method. (See also § 164, et seq.) The addition of gelatine as directed by him introduces only a slight source of error, which may be generally neglected.

VIII. Chlorinated Lime.-Löwenthal ${ }^{1}$ has titrated with chlorinated lime in the presence of indigo carmine in the same way as with permanganate of potassium, but the estimations generally yield too high results in consequence of the impurities present.

$\mathrm{Cech}^{2}$ has already expressed an unfavourable opinion of the propositions of Commaille ${ }^{3}$ and Millon ${ }^{4}$ to make the separation of iodine from iodic acid by tannin the basis of a method for its quantitative estimation. The decolourization of a solution of iodine by tannic acid in the presence of carbonate of soda has been recommended by Jean ${ }^{5}$ for the quantitative estimation of tannin. He states that 1 part of gallo-tannic acid decolourizes 4 parts of iodine, and reserves to himself the determination of the value of other tannins in terms of iodine. He admits that gallic acid also acts upon iodine, and advises, when both are present, first to make a total estimation, and then determine the gallic acid alone in a second portion of the liquid, after the tannic acid has been removed by gelatine or hide. The solution of tannic acid for standardizing should contain 1 part in 1,000 of water. Before titrating, 2 cc. of a 25 per cent. solution of cryst. carbonate of soda should be added for every $10 \mathrm{cc}$. of tannic acid solution. It must be observed that here, too, many organic compounds would act in a similar manner to tannin.

IX. Oxidation.-For the estimation of tannin Mittenzwey ${ }^{6}$ has availed himself of the fact that an alkaline solution of tannic acid rapidly absorbs oxygen from the air. In the analysis of plants this method will seldom be of any value. Cech has already shown that it yields unsatisfactory results with the tannins usually employed.

X. Titration with Cinchonine.-Wagner ${ }^{7}$ has proposed titration with sulphate of cinchonine, using acetate of rosaniline as an

1 Loc. cit. ${ }^{2}$ Loc. cit.

4 Annales de Chimie et de Phys. [3], xii. 26.

3 Compt. rend. lix. 599 (1864).

5 Zeitschr. f. anal. Chem. xvi. 123 (1877).

6 Journ. f. pr. Chemie, xci. 81, and Zeitschr. f. anal. Chemie, iii. 484 (1864).

See also Terreil, Zeitschrift des österr. Apothekervereins, Jg. xii. 377 (1874).

7 Zeitschr. f. anal. Chem. v. 1 (1866). See also Salzer, ibid. vii. 70 (1868);

Buchner, ibid. 139 ; Clark, Amer. Journ. Pharm. xlviii. 558 (1876). 
indicator. But the majority of those who have worked the process have failed to obtain good results. Almost all of them have found that the assumption that rosaniline would not colour the liquid until all the tannin had been precipitated by the cinchonine, was true of certain tannins only, and not of all. It has been shown that, with some tannins, the appearance of a red tinge in the solution, which is said to indicate the end of the reaction, may be noticed long before all the tannic acid has been precipitated. In many cases better results might be obtained with cinchonine if the tannic acid were precipitated by an excess, the liquid filtered and the excess of cinchonine in the filtrate determined by titration with potassio-mercuric iodide. This method has been adopted by Clark in estimating the tannic acid in tea. (See $\$ 65$.)

XI. Gelatine and Hide.-The behaviour of gelatine and hide to tannic acid is often made use of in the estimation of tannin. The estimation may be made either by determining the increase in weight of a piece of hide, previously freed from substances soluble in water and petroleum spirit by digestion in those solvents, when allowed to lie for some time in the solution of tannin, or by ascertaining the specific gravity of the solution before and after the absorption of the tannic acid, and calculating the amount from the difference. Hammer ${ }^{1}$ has constructed a table for gallo-tannic acid, from which the amount of tannin can be directly read off. If this method of estimation is to be adopted, a similar table would have to be constructed for other important tannic acids, showing the relation between the difference in specific gravity and the amount of tannin present.

XII. Gelatine: Gravimetric Process.-Precipitation of the tannin by gelatine, and calculation of the amount present from the weight of the precipitate, has also been tried. But the disadvantages which present themselves here are that these precipitates are neither sufficiently insoluble nor constant enough in composition to allow of their being made the basis of a gravimetric estimation; especially in washing the precipitate with pure water, considerable quantities of tannic acid are removed.

It is, therefore, most advantageous to apply the precipitation

${ }^{1}$ Journ. f. pract. Chem. clxxxi. 159. See also Löwe, Zeitschr, f. anal. Chem. iv. 365 (1865), and Hallwachs and Cech (loc. cit.). Davy has already estimated tannic acid gravimetrically by employing hide (Chem. News, 1863, p. 54, and Zeitschr. f. anal. Chem. ii. 419). 
with gelatine in the following manner: A solution of that substance-the value of which, in terms of the tannic acid to be estimated, has been previously ascertained-is run into the solution in which tannin is to be determined as long as precipitation occurs. The gelatine solution should be mixed with some salt, diminishing the solubility of the tannate of gelatine. For the latter purpose the addition of alum has been recommended (Miiller ${ }^{1}$ ). The proposal of Schulze ${ }^{2}$ to use chloride of ammonium, or of Löwenthal to add common salt and $\frac{1}{10}$ vol. of hydrochloric acid (specific gravity 1.12), appears better. A solution of gallotannic acid may be saturated with these salts; but in the case of other tannins (from oak, willow, and elm bark) a smaller quantity might be preferable. If Löwenthal's modification be aclopted, it is advisable to stir the liquid vigorously for five minutes after each addition of the gelatine solution. It has already been determined by Günther that the various tannins differ in the amount of gelatine they are capable of precipitating. He found that 100 parts of gelatine precipitate, in the presence of chloride of ammonium, 77 parts of gallo-tannic acid (according to Johanson 120 parts of dry tannic acid), 132 (Lehmann, 139) of catechu-, 130 kino-, 130 to 132 rhatania-, 130 oak-bark-, and 168 of tormentilla-tannic acid. As is well-known, gallic and catechuic acids do not precipitate gelatine.

§53. Gallic and Catechuic Acids.-If one of these two substances is to be looked for, the tannic acids should be first precipitated by gelatine, the excess of gelatine by alcohol; and after the alcohol has been removed by distillation under diminished pressure, gallicor catechuic acid may be isolated by shaking with ether or acetic ether. If care has been taken to avoid using a large excess of gelatine, the treatment with alcohol might be omitted; and in many cases it would be possible to agitate even the aqueous solu-

${ }^{1}$ Archiv d. Pharm. xxxviii. 147 (1845). Gauhe did not succeed in his endeavour to find an indicator (iodide of starch) to show the final reaction in titrating. Compare Zeitschr. f. anal. Chem. v. 232 (1866). Neither was Cech quite satisfied with an iron solution used for the same purpose. See also Hallwachs (loc. cit.).

${ }^{2}$ Zeitschr. f. anal. Chem. v. 455 (1866). Compare also Salzer, ibid. vii. 70 (1868), and Johanson, 'Beitr. z. Chemie der Eichen, Weiden, und Ulmenrinde,' Diss. Dorpat, 1875 , pp. 72, 76. Also Lehmann (loc. cit.). A more recents critical review of the more important methods for estimating tannic acid by Löwenthal will be found in the Zeitschr. f. anal. Chemie, xvi. 33, and 201 $(187 \%)$, and xx. 91 (1881). 
tion $(\S 48)$ directly with ether, renewing the solvent four or five times. On evaporating the ethereal solution, both gallic and catechuic acids remain behind in a crystalline form, generally needles felted together. (Cf. $\$ 151,165$.)

The weight of the dried residue frequently indicates with tolerable accuracy the quantity of the substance present; but if the residue be mixed with much colouring or amorphous matter, so as to cause some hesitation in accepting the weight as correct, the result obtained may be verified by titration with permanganate of potash. (See above.) If the material has been extracted with ether previous to treating with alcohol, gallic and catechuic acids will be found in the aqueous solution from the ethereal extract. (Cf. $\S 38,151$.)

For the free vegetable acids which may occur in the alcoholic extract see $\S 82$. (See also in $\S 159$.)

\section{EXAMINATION FOR GLUCOSIDES, BITTER PRINCIPLES,}

ALKALOIDS, ETC.

§ 54. Extraction by Agitation.-If no tannic acid or allied substance has been found in the aqueous liquid ( $\$ 48)$, but by the bitter taste or other properties the presence of a bitter principle, glucoside or alkaloid insoluble in ether but soluble in water is suspected, the watery solution prepared from the evaporation residue of the alcoholic tincture may be subjected to consecutive treatment with various liquids which, being themselves insoluble in water, are adapted for removal of substances in solution by agitation and separation. The aqueous solution from the ethereal extract (\$38) may also be treated in a similar manner. The use of petroleum spirit, benzene, and chloroform may be especially recommended for this purpose; they should be employed in the order in which they are named, and the liquid should be rendered first slightly acid with sulphuric acid, and subsequently alkaline with ammonia; I have spoken at length on this subject in my 'Ermittelung der Gifte.' After each agitation, the solvent should be separated, washed once by shaking with pure water, again separated, evaporated to dryness, and the residue examined. If a solvent, as for instance petroleum spirit, removes any appreciable quantity of a substance, the agitation with this liquid should be

${ }^{1}$ P. 119. Compare also Russ. Archiv für gerichtl. Med. J. i. und Pharm. Zeitschr. f. Russland, v. 85 ; vi. 663. 
repeated until only traces of the substance are dissolved. Then, and not till then, the same treatment is repeated with the next solvent, and so on. All liquids employed for agitation must be rectified shortly before being used. Petroleum spirit must be as volatile as possible; benzene should boil constantly at $81^{\circ} \mathrm{C}$, and yield nitro-benzene when treated with fuming nitric acid.

\$ 55. From Acid Solution.-Of the better-known bitter principles, acids and alkaloids removed by petroleum spirit from an acid solution, the following may be mentioned :

Salicylic acid (cf. § 26). Pungent principles of capsicum, etc. (\$126). (Both of these would have been already detected in the ethereal extract. Salicylic acid may be more easily removed by benzene or ether.) Piperin-the majority of this principle will be found in the part of the alcoholic extract insoluble in water (compare further $\S$ 171, 178). Absynthin, cannot be completely removed by petroleum spirit (\$ 156). Hop-resin (\$156).

Benzene removes from the same solution santonin (cf. $§ 154$ ); curyophyllin (\$ 156); cubebin (\$155); digitalin (remains principally in that part of the ethereal extract which is insoluble in water (cf. $§ 155)$; gratiolin (\$ 167); cascarillin (§ 156); elaterin (§ 156); populin (\$167); colocynthin (\$ 167); absynthin (\$ 156); quassin (\$156); menyanthin ( $(167)$; ericolin ( $\$ 155)$; daphnin (\$167); bitter principle of Cnicus benedictus (\$ 168); caffeine (\$§ 171, 176); piperin (see above); colchiceine ( $\$ 171)$; berberine is dissolved by benzene in small proportion only (compare $§ 171$ ).

Besides the substances already named as being dissolved by petroleum spirit and benzene, chloroform removes also among others: Benzoic acid (cf. $§ 26$ ); digitalein (sparingly soluble in ether, $\$ 155$ ); convallamarin ( $\$ 167)$; saponin (insoluble in ether, difficultly soluble in absolute alcohol, $\S 77$ et seq., 167); senegin (the same); physalin ( $(167)$; syringin ( $\$ 167)$; asculin ( $\$ 167)$; picrotoxin (\$155); helleboreïn (\$ 167); cinchonine (is insoluble in ether, $\S 171,182,184)$; theobromine (\$ 177); papaverine (\$171); narceïne (\$ 171). Colchicine, solanidine, quebrachine, yeissospermine.

\$56. From Alkaline Solution.-After the last agitation with chloroform the aqueous liquid should be shaken whilst still acid with petroleum spirit. This removes the small quantity of chlnroform remaining dissolved by the watery liquid. An error 
might be made in omitting this treatment, since on rendering alkaline and shaking with petroleum spirit this solvent would take up a little chloroform and would consequently be no longer pure. After, therefore, the remainder of the chloroform has been removed by petroleum spirit, the liquid may be made alkaline with ammonia, and the agitation with the same solvents repeated in the same order. In addition to these three liquids I have, however, after agitation with chloroform, employed amylic alcohol for detecting certain poisons. It removes morphine, solanine, (§ 171), salicine ( $\$ 167)$, and some other substances from their aqueous solutions with special facility.

It is principally alkaloids that are removed by petroleum spirit, etc., from ammoniacal solution. Petroleum spirit dissolves, for instance, traces of strychnine, brucine, emetine, veratine, sabadilline, and sabatrine. All these substances are, however, more easily and completely taken up by benzene and chloroform.

But petroleum spirit is specially valuable in the examination for the so-called volatile and, at ordinary temperatures, liquid alkaloids, such as coniine, methylconiine (and conhydrine), nicotine, lobeliine, sparteïne, alkaloids in pimento, capsicum and Sarracenia purpurea. Aniline, trimethylamine, and allied substances are also dissolved by it (\$§ 171,239). In examining for volatile alkaloids I have advised agitation of the aqueous liquid with petroleum spirit, and evaporation of the solvent, after separation, at a temperature of about $20^{\circ}$, on glass dishes previously moistened with strong hydrochloric acid, on which the hydrochlorides of the alkaloids will partly, at all events, remain behind. A freshly-prepared dilute solution of hydrochloric acid gas in ether may be advantageously substituted for the usual aqueous acid.

Benzene removes from ammoniacal solution, in addition to the alkaloids already mentioned, atropine, hyoscyamine, physostigmine, pilocarpine, gelsemine, taxine, quinidine, narcotine, codeine, thebaine, delphinine and delphinoidine, aconitine, aspidospermine, pereirine, and a trace of cinchonine. (Cf. $\$ 171$.

In addition tu these, again, chlornform dissolves from ammoniacal solution cinchonine, papaverine, narceine, nupharine, the alkaloids of ielandine, and small quantities of morphine (\$171).

$\$ 57$. Direct Tests for Glucosides, Alkaloids, etc.-The number of acids, glucosides and alkaloids (cf. $\S 21$ ) that may be isolated by 
this method of agitation is doubtless very large, and by making the required experiments the list given in the preceding section might be rendered far more complete. It is this very fact that renders the method so suitable for the qualitative examination of those plants and parts of plants the constituents of which are at present unknown. Of course, it is possible to employ infusions which have been prepared by digesting the material under examination with water on the water-bath, instead of aqueous solutions from the ethereal or alcoholic extracts. This would especially be the case if acids, bitter principles and glucosides are to be looked for. Alkaloids may likewise be tested for by digesting the material with water acidulated with sulphuric acid (1 in 50). In both cases, however, it must be remembered that by thus directly extracting the substance with aqueous liquids many bodies, such as mucilage, etc., are dissolved, and that this is avoided by treating them according to the method first described. The presence of such substances is disadvantageous, inasmuch as they sometimes render the extraction of a principle from aqueous solution by the method of agitation more difficult, and always act injuriously in rendering the separation of the two liquids after shaking almost impossible. It is therefore advisable to remove all matter tending to increase the viscosity of the aqueous infusion by concentrating to a syrupy consistence (if necessary, after having previously nearly neutralized with ammonia or magnesia), precipitating with about three volumes of spirit, filtering after standing twelve to twenty-four hours in a cold place, and distilling off the alcohol.

§ 58. Alkaloids, etc., not Removable by Agitation.--Some bitter principles, glucosides and alkaloids cannot, however, be removed from solution by agitation, either because they have less tendency to pass into any other known liquid than to remain in aqueous solution, or because they are insoluble in water. The latter is the case, for instance, with the glucosidal resins which occur in the convolvulaceæ. Such substances are generally isolated with the resins. (Cf. § 153.)

The purification of bitter principles and glucosides that are soluble in water, but cannot be removed by shaking, may be effected by evaporating the aqueous solutions prepared from the ethereal or alcoholic extracts, and repeatedly dissolving the substance in chloroform, alcohol, or ether. It will be found easier to 
purify a bitter principle dissolved by water from the ethereal extract, than one obtained in a similar manner from the alcoholic extract, since the latter contains glucoses and tannins, which are insoluble in ether. Apart from the treatment with chloroform and other solvents which I have just described, another method of purification may in this case be adopted-viz., evaporation of the aqueous solution, extraction with the smallest possible quantity of absolute alcohol, and precipitation of sugar, etc. with ether.

§ 59. Separation of Tannin.-Tannic acid, when present in solution, together with bitter principles, etc., may frequently be removed by digesting the aqueous infusion with oxide or hydrate of lead. If salicine (cf. $\S 167$ ), for instance, is to be separated from tannin, the aqueous infusion may be mixed with oxide of lead, evaporated to dryness on the water-bath and extracted with alcohol. Basic acetate of lead may also be occasionally used, when a bitter principle is to be separated from tannin, vegetable acids, albuminous matter and the like. Of course, it must have been previously ascertained that the bitter principle in question is not precipitated by lead; should that be the case, it may sometimes be isolated by decomposing the lead compound with sulphuretted hydrogen. By combining a bitter principle with lead a separation may sometimes be effected from sugar, etc. This method is, however, inapplicable if tannic acid be present, when it will often be found advisable to precipitate vegetable acids, tannin, etc., by neutral acetate of lead before throwing down the bitter principle, etc., with the basic salt ( $\$ 51,162)$.

$\S 60$. Separation of Lead Precipitate.-If such compounds of lead with bitter principles, glucosides, etc., are to be washed with water, it is very advisable to effect this as rapidly as possible by decantation. Such precipitates often block a filter, or form, by contraction, channels through which the wash-water runs off without penetrating the precipitate. If the use of a filter is necessary, repeated suspension in water and filtration is advisable. The washing of such precipitates should not be continued too long, as they usually undergo decomposition during the process and yield the bitter principle to the wash-water. The presence of carbonic acid in the water used for washing is specially to be avoided.

Decomposition.-The decomposition of these precipitates is usually effected by sulphuretted hydrogen, which, however, does 
not act well if the lead eompound has been previously dried. It is a well-known fact that, in the course of this operation, the bitter principle may be mechanically retained by the sulphide of lead formed. To avoid loss in this way the sulphide may be filtered off, washed, dried, powdered, and boiled with alcohol. In evaporating the alcoholic extract care should be taken not to confound crystals of sulphur with the bitter principle, etc. It will often be found advantageous to decompose the lead precipitate in alcohol instead of water. The surface-attraction of the sulphide of lead will, nevertheless, be frequently found useful in retaining foreign bodies, such as colouring matter and the like, whilst bitter principles, etc., pass into solution.

The directions given for the decomposition of the lead precipitates may also be followed in isolating tannic and vegetable acids from such compounds. (See also $§ 162$.

$\S 61$. Glucosides; Recognition.-In proving the glucosidal nature of a substance advantage may be taken of the influence exercised by ferments (saliva, emulsin, myrosin), etc., or dilute acids (accompanied by heat) on glucosides, which, under such circumstances, split up and yield sugar as one of the products of decomposition. It is advisable to ascertain whether the substance itself, in as pure a state as possible, reduces an alkaline solution of copper, either at the ordinary temperature or on boiling. If no reduction takes place the further examination for glucose is much facilitated. It is customary to boil the substance under examination with water containing 1 to 2 per cent. of sulphuric or hydrochloric acid, and test the liquid for sugar from time to time. The rapidity with which decomposition may be thus effected varies very greatly. Some glucosides yield a sugar reaction after boiling for a few minutes only; others require several hours. In some cases it is preferable to allow the acid to act under pressure, or in alcoholic instead of aqueous solution. (Cf. $\$ 153,160$.)

The decomposition-products which are formed, together with glucose, from glucosides, are not unfrequently insoluble in water, and therefore render the liquid turbid in proportion as the reaction proceeds. This peculiarity may be often made use of as proof that decomposition has commenced, especially in those cases in which the glucoside itself reduces Fehling's copper solution. After the completion of the reaction and the cooling of the liquid, the decomposition-product may be filtered off and further 
examined. If, on the other hand, this substance be soluble in water, its isolation may be attempted by the method of agitation.

An experiment should also be made to ascertain the action of the glucose thus produced on a ray of polarized light, as well as its behaviour with yeast-that is, its capability or incapability of entering into fermentation. For this purpose it is best to decom. pose the glucoside with sulphuric acid, which may be subsequently removed by carbonate of barium. The filtrate from the sulphate of barium, which should be faintly acid in reaction, should be mixed with a little yeast, introduced into a eudiometer over mercury, and observation made whether, under these circumstances, carbonic-acid gas is evolved. Many glucosides yield, besides glucose, products of decomposition which are antagonistic to alcoholic fermentation; these are, if possible, to be removed.

This fermentation experiment will have a particular value in all cases in which the glucoside itself reduces alkaline copper solution. Proof of the glucosidal nature of the substance may then be found in the experiment yielding a negative result before, but a positive one after, the action of the dilute acid, especially if the substance be soluble in ether or cold absolute alcohol. Saccharoses and other carbohydrates, which would yield similar results, are thus excluded, they being insoluble in the liquids named.

It is hardly necessary for me to point out the desirability of estimating, by means of Fehling's copper solution, the sugar produced by the decomposition of a glucoside. (Cf. $\S 83$, et seq.; $\S 200$, et seq.)

Some bodies which are usually treated of with the glucosides yield, when acted upon by acids, not glucose, but substances allied to sugar or mannite, which, like isodulcite, are unfermentable. (Cf. $§ 212$.

§62. Sulphuric Acid Group-reaction.-Many glucosides are capable of acting like sugar when mixed with bile and sulphuric acidthat is, of producing a red colour. This reaction has been described as to a certain extent characteristic of the whole group of glucosides; but it should be remarked that among them there are many which are reddened by sulphuric acid alone, whilst some cannot replace sugar in the test for bile; and others, when mixed with sulphuric acid, assume such characteristic colours that the bile reaction is quite undistinguishable.

For further information concerning glucosides, compare $\$ 165$, et seq. 
§ 63. Isolation of Alkaloids, etc., not Removable by Agitation.-As stated in $\$ 58$, there are some alkaloids which, for the reasons there given, cannot be isolated by the method of agitation. To scparate these in a state of purity the extracts prepared according to $\$ 57$ should be exhausted as far as possible by shaking with petroleum spirit, etc., and then evaporated to dryness. The dry residue should be finely powdered (if necessary, with the addition of washed sand or siliceous earth) and treated with alcohol, ether, chloroform, etc. The exhaustion, however, with these solvents will be incomplete if the residue be not reduced to a very fine powder. (See also $§ \S 65,66$.)

Detection by Group-reagents.-Before proceeding to this extraction it may appear desirable to ascertain whether an alkaloid is present at all. To that end the liquid obtained in \$ 57, containing sulphuric acid but no alcohol, may be tested for alkaloids by precipitants which have been introduced as group-reagents for that class of substances. The following may be especially recommended :

Tri-iodide of potassium - that is, an aqueous solution of iodine in iodide of potassium-gives, with aqueous solutions of most alkaloids, amorphous precipitates of a dark-brown or kermesmineral colour, and is one of the most delicate reagents. An alcoholic solution of an alkaloid frequently remains clear on the addition of the tri-iodide; or if a precipitate is formed, it differs in properties from that produced in aqueous solutions. For example, berberine and narceine would under these conditions yield crystalline precipitates.

Tribromide of potassium, prepared in a similar manner, also precipitates some of the alkaloids from very dilute solutions, but, in addition, forms yellowish compounds with phenol, orcin, and many allied bodies ( $\$ 158)$.

Potassio-mercuric iodide, obtained by decomposing mercuric chloride with an excess of iodide of potassium, yields with most alkaloids, white, flocculent precipitates, which sometimes gradually assume a crystalline character. (See also $\S 65$.) The presence of free acid may sometimes cause a difference in the precipitate obtained from one and the same alkaloid.

Potassio-bismutlic iodide, prepared by dissolving iodide of bismuth and iodide of potassium in water, yields, even in highly dilute solutions, precipitates which are very sparingly soluble, 
and resemble orange sulphide of antimony in colour. It should not, however, be forgotten that albuminous and other similar substances are also precipitated by this reagent. (Cf. § 232.)

Potassio-cadmic iodide, obtained in analogous manner from iodide of cadmium, gives white precipitates, which, like those yielded by potassio-mercuric iodide, sometimes become crystalline. They are mostly rather more soluble than those produced with the latter reagent.

Phospho-molybdic acid (a solution of the sodium salt in nitric acid) yields with most alkaloids yellowish precipitates, which are in certain instances rapidly reduced, and assume a bluish or greenish colour. Ammoniacal salts and less complex amidecompounds are also precipitated by this reagent.

Metatungstic acid gives similar precipitates ( $\$ 177)$.

Chloride of gold yields yellowish precipitates with very dilute solutions of many alkaloids. Sometimes a rapid reduction takes place, and the yellowish colour changes to a reddish-brown, the liquid itself occasionally assuming at the same time an intense reddish tint ( $\$ 186)$. I consider this reagent especially valuable for our purpose, as ammoniacal salts and the less complex amides are not precipitated by it.

Perchloricle of platinum forms brownish-yellow precipitates with most alkaloids (not all), but is less valuable than chloride of gold, because the precipitates are mostly more soluble, and because it forms sparingly soluble compounds with ammonipm and potassium salts, etc. The precipitates obtained with this reagent also sometimes show a disposition to decompose.

Mercuric chloride. - The white precipitates which this salt yields with alkaloids are not very sparingly soluble, but it possesses some value, as it does not precipitate ammoniacal salts, etc. The same is the case with

Picric acid, which gives yellowish precipitates.

Tannic acid, the compounds with which are usually of a greyishyellow or greyish-brown tint, and

Bichromate of potash, which yields yellowish and occasionally crystalline salts. ${ }^{1}$

1 For group-reagents for alkaloids see further in my Ermittelung ron Giften, 2nd edition, 123; also Selmi, Jahresb. f. Pharm. 1874, 480; 1875, $341 ; 1876,628$ (Year-book Pharm. 1876, 110). For behaviour of cinchonaalkaloids to sulphocyanide of potassium compare Schrage, Arch. d. Pharm. 
To confirm the presence of an alkaloid, advantage may also be taken of the fact that they all contain nitrogen, and, therefore, yield Prussian blue with Lassaigne's test (heating with metallie sodium, etc.). This test will be specially valuable if another peculiarity of most, but not all, alkaloids-viz., the alkaline reaction towards litmus and capability of forming salts-be not well defined (colchicine), or if a compound be obtained which must be referred to the group of amido-acids (colchicine) or glucosidal alkaloids (solanine). It must not, however, be forgotten that some of the glucosides already mentioned contain nitrogen ( $\$ 167)$. For tables of the colour-reactions characteristic of many alkaloids see $\S 171$.

\$64. Alkaloids not Isolated by the Method of Agitation; Purification.-In cases in which an alkaloid is present that cannot be separated in this way or purified as recommended in $\S 63$, the following method may be tried. The alkaloid is precipitated by potassio-mercuric iodide from its solution in water acidulated. with dilute sulphuric acid, the precipitate filtered off, washed, suspended in water and decomposed by sulphuretted hydrogen. On filtering off the sulphide of mercury, a solution of the hydriodate of the alkaloid together with free hydriodic acid is obtained. Sulphate of silver is then added as long as it causes a precipitate, and the iodide of silver filtered off. After removing the sulphuric acid by addition of caustic baryta and filtration, a solution of the alkaloid may be obtained by freeing the filtrate from excess of baryta by carbonic-acid. gas. The last separation of baryta, however, is not always quite complete. It might be better therefore in many cases to remove the sulphuric acid by carbonate instead of hydrate of barium. The former, moreover, would be less likely to decompose the alkaloid.

In following this method, inconvenience is occasionally experienced in filtering off the sulphide of mercury, which sometimes separates in a very finely-divided state. To obtain a clear filtrate,

clxxiv. 143; [3], v. 504; xiii. 25; Hesse, ibid. xii. 313 ; xiii. 481; Todeffroy, Oesterr. Zeitschr. f. Pharm. 1878, Nos. 1 to 12 (Am. Journ. Pharm. 1878, 178). For the action of silico-tungstic acid on alkaloids see Godeffroy, Archiv d. Pharm. ix. 434; chloride of antimony and stannous chloride see Godeffroy, ibid. 147, and Smith, Jahresb. f. Pharmacie, 1879, 166 ; arseno-molybdic acid, selenic and telluric acid, Brandt, Jahresb. f. Pharmacie, 1875,341 . Smith heats trichloride of antimony and projects the alkaloid into the fused mass. Morphine and codeine produce a greenish, narcotine olive. green, thebaine, brucine and veratrine, red colouration. 
evaporation with white bole and re-solution in water may be tried. The iodide of silver and sulphate of barium are also at times very difficult to remove, and clear liquids can only be obtained by repeated filtration through double filters.

Should the alkaloid, after liberation with caustic baryta, be sparingly soluble in water, it may be precipitated simultaneously with the sulphate of barium. In this case it may be extracted from the dry precipitate by treatment with alcohol or other suitable solvent. But those alkaloids that resist extraction by the method of agitation are generally freely soluble in water.

Many alkaloids, too, are easily attacked by alkalies, splitting up, on boiling, into acids and new complex amides. Atropine under such circumstances yields tropine and tropic acid; hyoscyamine is resolved into the same two substances. (Cf. § 65.) How easily errors are thus caused may be seen from the number of alkaloidal substances that have been described in text-books as special alkaloids, and which are in reality nothing but products of decomposition (acolyctine and napelline = aconine; lycoctonine $=$ pseudaconine). ${ }^{1}$ Curarine is another alkaloid easily decomposed by alkalies. Certain members of this class are also decomposed by boiling with dilute acids.

If the alkaloid under examination is not easily attacked by baryta or lime, it may be precipitated by phospho-molybdic or phospho-tungstic acid ( $\$ 63)$, and separated from its combination with either of these acids by baryta or lime, the excess of alkaline earth being removed by carbonic acid. These methods, which are sometimes of use in the quantitative estimation of certain alkaloids, will be discussed in detail in $§ 177$.

$\S 65$. Estimation.-For the quantitative determination of alkaloids, one of the following methods may be feasible:

1. The alkaloid obtained in $\S 64$ may be dried and weighed.

2. The substance removed by agitation according to $\$ \S 55,56$, may be weighed, care being taken to avoid loss. ${ }^{2}$

I I avail myself of this opportunity to draw attention to the more recent researches of Wright and Luff on the aconite-alkaloids. See Jahresb. f. Pharm. 1873,$131 ; 1874,135 ; 1876,169 ; 1877,434 ; 1879,189$; and in Pharm. Journ. and Trans. On atesin of Aconitum heterophyllum see Wasowicz, Archiv d. Pharm. xiv. 193 (1879) (Pharm. Journ. and Trans. [3], x. 310). See papers by Wright and Luff, etc., in Pharm. Journ. and Trans. [3], vols. ix. x. and xi.

2 Compare the methods I have proposed for the quantitative estimation of trychnine, brucine, and veratrine, in $\S 174$. Günther has successfully em- 
3. The alkaloid may be precipitated from its aqueous solution by certain reagents, and estimated gravimetrically.

Chloride of gold, or sometimes perchloride of platinum (§ 173), may be advantageously used as precipitant in the last case, as the amount of alkaloid and chlorine present may be approximately calculated from the amount of gold or platinum contained in the precipitate. Alkaloids may often be estimated gravimetrically and volumetrically by precipitation with potassio-mercuric iodide (\$ 174).

I have discussed this subject fully in my 'Chemische Werthbestimmung starkwirkender Droguen,' I where I have shown that many alkaloids may thus be accurately estimated. I found, however, that the precipitates produced were not always analogous in composition, and that therefore the precipitating power or value of the unit-quantity of reagent must be determined for each single alkaloid. The composition of the precipitate yielded by one and the same alkaloid may vary with the concentration of the solution, and a difference in the amount of sulphuric acid present may sometimes influence the result. A large excess of acid is incompatible with the accurate estimation of certain alkaloids, such as brucine and coniine, whilst in other cases (nicotine, colchicine) it is necessary. The latter alkaloid, together with atropine and others, requires a considerable excess of the reagent for complete precipitation, and in its gravimetric estimation therefore this condition must obtain; on the other hand, the precipitate first produced is sometimes redissolved on the addition of an excess of the precipitant. With regard to the reagent itself, I may observe that, according to Mayer, it is not advisable to prepare it by dissolving iodide of mercury in iodide of potassium, the best method being to mix 13.546 gram of perchloride of mercury with 49.8 gram of iodide of potassium and water to make one litre.

For details of experiments I refer to the work already men-

ployed the method of agitation for the estimation of atropine. Compare Pharm. Zeitschr. f. Russland, 1869, p. 89 (Year-book Pharm. 1872, 236). In colchicum also it would be more advisable to determine the alkaloid by shaking with chloroform than by precipitating with potassio-mercuric iodide. The material should be extracted with pure water, and the solution made acid rather than alkaline before shaking with chloroform.

1 St. Petersburg, 1874. Schmitzdorff. 
tioned, and will only remark here that, as a rule, the material may be extracted with water acidulated with sulphuric acid, and that in many cases the estimation may be made in this liquid without further treatment. But if the presence of mucilaginous substances, etc., prevent this, and their partial removal by alcohol be necessary, the solution must be completely freed from spirit before titrating. The termination of the reaction is usually found by a drop of the filtered solution yielding no precipitate with a drop of the reagent.

Aconitine and Nepaline.-1 cc. of potassio-mercuric iodide solution of the above strength indicates 0.0269 gram of aconitine, and the precipitate (if the estimation be made gravimetrically) has the composition $\mathrm{C}_{27} \mathrm{H}_{40} \mathrm{NO}_{10} \mathrm{I}_{2}+\mathrm{HgI}_{2}{ }^{1}$ In the latter case a correction of 0.00005 gram of aconitine must be made for every cc. of solution.

1 cc. of potassio-mercuric iodide indicates 0.0388 gram nepaline (pseudaconitine).

Atropine. - 1 cc. is equivalent to 0.0097 gram atropine if the solution contain about 1 in 200 , but in solutions containing 1 in 330 it is equivalent to only 0.00829 gram. The precipitate obtained by adding an excess of the precipitant to a solution containing about 1 in 200 to 300 has the composition $\left(\mathrm{C}_{17} \mathrm{H}_{24} \mathrm{NO}_{3} \mathrm{I}\right)_{2}+\mathrm{HgI}_{2}$. In working with solutions containing 1 in about 350 to 500 a correction must be made of 0.00005 gram of atropine for every cc. of filtrate ( $\$ 174$ ).

Hyoscyamine. -1 cc. of the mercury solution is equivalent to 0.00698 gram hyoscyamine, the concentration being about 1 in 200. According to the more recent researches of Ladenburg henbane contains two alkaloids, one of which is isomeric with atropine, and identical with daturine and duboisine. Compare Berichte d. d. chem. Ges. xiii. 909, 1081, 1340, 1549 (Pharm. Journ. and Trans., [3], x. 759, 789, 790). [Ladenburg distinguishes hyoscyamine from atropine by the melting-points of the alkaloids and their gold salts. In belladonna a second alkaloid at least is present, which is possibly identical with hyoscyamine. ${ }^{2}$ The second alkaloid in henbane has been named hyoscine by Ladenburg. This must not, however, be confounded with

1 For the present I make use of the old formula for aconitine, as the new does not agree so well with the volumetric determinations.

${ }^{2}$ Compare Kraut, Ber. d. d. chem. Ges. xiii. 165. 
the hyoscine of Höhn and others. Its gold salt melts at a higher temperature than that of atropine or hyoscyamine.] (See further in $\S 174$.)

Emstine. -1 cc. of the potassio-mercuric iodide precipitates 0.0189 gram emetine. The precipitate has the composition $\mathrm{C}_{20} \mathrm{H}_{32} \mathrm{~N}_{2} \mathrm{O}_{5} \mathrm{I}_{2}+\mathrm{HgI}_{2}{ }^{1}$

Coniine. - 1 cc. indicates 0.0125 gram. coniine provided that the solution contain $\frac{1}{4}$ to 1 per cent. of the alkaloid, as little free acid as possible, and, in addition, 3 to 4 per cent. of chloride of potassium. If these conditions are complied with the composition of the precipitate will be $\left(\mathrm{C}_{18} \mathrm{H}_{16} \mathrm{NI}\right)_{2}+\mathrm{HgI}_{2}(\$ \$ 174,180)$.

Nicotine. -1 cc. indicates 0.00405 gram nicotine; the composition of the precipitate is $\mathrm{C}_{10} \mathrm{H}_{10} \mathrm{~N}_{2} \mathrm{I}_{2}+\mathrm{HgI}_{2}$.

Strychnine and Brucine. - 1 cc. precipitates 0.0167 gram strychnine and 0.0197 gram; anhydrous brucine (in the latter case as little free acid as possible should be present). The precipitates have the composition $\mathrm{C}_{21} \mathrm{H}_{22} \mathrm{~N}_{2} \mathrm{O}_{2} \mathrm{HI}+\mathrm{HgI}_{2}$, and $\mathrm{C}_{23} \mathrm{H}_{26} \mathrm{~N}_{2} \mathrm{O}_{4} \mathrm{HI}+\mathrm{HgI}_{2}(\S \S 174,180)$.

Colchicine. -1 cc. precipitates 0.0317 gram colchicine, the concentration being about 1 to 600 , and the solution containing 7 to 10 per cent. of sulphuric acid. The precipitate appears to contain four equivalents of colchicine to one of $\mathrm{HgI}_{2}$.

Morphine and Narcotine.-1 cc. corresponds to 0.02 gram crystallized morphine ${ }^{2}$ and 0.0213 gram narcotine. (See also $\$ 174$.)

Veratrine, sabadilline, and sabatrine. - 1 cc. indicates, according to Masing, ${ }^{3}$ 0.0296 gram veratrine. Little sulphuric acid only should be present and a correction of 0.000068 gram veratrine made for every cc. of liquid. According to the same chemist, $1 \mathrm{cc}$. of the potassio-mercuric iodide is equivalent to $0.0374 \mathrm{gram}$ sabadilline and 0.03327 gram sabatrine. Correction for every cc. 0.00005 gram of the former and 0.0000408 gram of the latter (§ 174).

Physostigmine. - 1 cc. precipitates 0.01375 gram physostigmine (Masing). Correction for every cc. 0.000105 gram. The composition of the precipitate is assumed to be $\mathrm{C}_{15} \mathrm{H}_{21} \mathrm{~N}_{3} \mathrm{O}_{2} \mathrm{HI}+\mathrm{HgI}_{2}$. (See also § 174.)

1 This formula, also, will have to be altered as soon as analyses of the pure emetine prépared by Podwissotzki are published.

2 For the application of potassio-cadmic iodide to the quantitative determination of opium alkaloids see Lepage, Repert. f. Pharm. 1875, 613.

${ }^{3}$ Archiv d. Pharm. ix. 310 (Journ. Chem. Soc. xxxii. 369). 
Berberine.-1 cc. indicates 0.0425 gram berberine (Beach). ${ }^{1}$ The precipitate is stated to contain almost exactly half its weight of pure berberine.

Quinine.-According to Prescott ${ }^{2}$ the double salt of quinine with iodide of mercury contains 34.5 per cent. of alkaloid, and is almost insoluble in water. From the recent researches of Hielbig? it would appear that no special advantage is to be looked for in the application of potassio-mercuric iodide to the quantitative estimation of quinine.

Chelidonine and Sanguinarine.-From some experiments made by Masing I anticipate that $1 \mathrm{cc}$. of potassio-mercuric iodide will be found to indicate 0.01675 gram of the former and $0.01485 \mathrm{gram}$ of the latter. ${ }^{4}$ (Compare also $§ 174$ et seq.)

$\S 66$. Estimation of Theine, etc.-The quantitative determination of some alkaloids may be made as follows : The material is boiled with water, either pure or containing a little sulphuric acid, the filtered or strained decoction evaporated with magnesia or lime, ${ }^{5-}$ and the residue finely powdered with sand or some other inert. substance. It is then extracted with ether, chloroform, or other suitable solvent, the filtered solution evaporated and the residue weighed. ${ }^{6}$ I have found this method well adapted for estimating the alkaloid in tea (without the addition of acid).

Similar methods have also been proposed for the estimation of the total alkaloid in cinchona-bark, but the long-continued action of the dilute acid necessary to dissolve the alkaloid appears to decompose part of it and renders the estimation inaccurate. (Compare $\S 176$.

$\S 67$. Extraction of Cinchona Alkaloids.-In a series of experiments in my laboratory the following method was found by Hielbig ${ }^{7}$ to be the most advantageous : 25 grams of powdered bark are digested with 100 grams of 1 per cent. sulphuric acid at the ordinary temperature for twenty-four hours, care being taken to ex-

1 American Journ. Pharm. xlviii. 386.

2 Ibid. xlix. 482.

3 Kritische Beurtheilung, etc. Diss. Dorpat.

4 Compare my Chemische Werthbestimmung, p. 101. Also Naschold, Journal f. prakt. Chem, cvi. 385.

${ }^{5}$ Compare Cazeneuve, Jahresb. f. Pharm. 1875, 342.

${ }^{6}$ Compare also Lösch, Pharm. Zeitschr. f. Russland, xviii. 545, and my remarks on his method, Jahresb. f. Pharm. 1879, 165 (Year-book Pharm. $1880,60)$.

7 Loc. cit. 
clucle direct sunlight; 500 cc. of spirit are poured in, and after two hours 25 grams of slaked lime are added to the mixture. The whole is then macerated for two days, and finally boiled $\mathrm{fcr}$ half an hour on the water-bath. To the filtrate, together with 100 cc. of wash-alcohol, the results of two following extractions, each with $250 \mathrm{cc}$. of spirit and 100 cc. washings, are added. The mixture is neutralized with 25 drops of dilute sulphuric acid ( 1 to 7 ) or more if much cinchonine is present. After standing twenty-four hours the spirituous solution is filtered, and the alcohol recovered by distillation, the process being stopped as soon as the liquid becomes cloudy (about $200 \mathrm{cc}$.) ; 15 cc. of 2 per cent. sulphuric acid are then added, and the evaporation continued on the water-bath, carbonization being carefully avoided. The residue is treated with water, the resin filtered off and washed with a little dilute sulphuric acid. The alkaloid is then precipitated by carbonate of soda, and the whole evaporated on the water-bath to about $20 \mathrm{cc}$. After cooling, the resinous precipitate is filtered off, rubbed down to a powder in a mortar with irater, retransferred to the filter, washed, dried, and weighed. From the filtrate and wash-water the alkaloid is extracted by chloroform, and the weight thus found added. For the separation of the more important bark alkaloids from each other, see $\S \S 183,184$.

- $\$ 68$. Estimation by Titration.-If the alkaloid under examination has a powerfully alkaline reaction, it may be separated by the method of agitation, or according to $\S \S 66,67$, and estimated by titration with $\frac{\mathrm{N}}{10}$ acid. A method of this kind has been proposed by Schlössing and others for the quantitative determination of nicotine in tobacco. ${ }^{1}$ (See $\left.\S \S 179,180.\right)$

$\S 69$. Separation of two or more Alkaloids. - In the methods described for the estimation of alkaloids it was assumed that only one was present. But two or more may be met with in the same plant. In attempting their separation their behaviour to solvents should be ascertained. Ether, for instance, may be used to separate quinine from cinchonine, narcotine from morphine, delphinine from delphinoidine.

${ }^{1}$ Annales de Chim. et de Phys. xix. 230 (Am. J. Pharm. xix. 68) ; also Wittstein and Brandt, Vierteljahresschrift f. prak. Pharm. xi. 351, and xiii. 322 ; Liecke, Zeitschr. f. anal. Chem. iv. 492 ; Kosutany, Anal. Best. einiger Bestandth. d. Tabakspflanze. Diss. Altenburg in Hungary, 1873. 
This method is not, however, always successful, and compounds of the alkaloids differing considerably in solubility, etc., must be looked for, applicable to the separation to be effected. Quinine may be separated from quinidine by precipitation with Rochelle salt, quinidine from cinchonine by iodide of sodium, etc. Use may also be made of the difference in equivalent weights. (Compare the estimation of brucine in presence of strychnine in $\S 174$.) See also $§ \S 180$ to 183.

EXAMINATION FOR GLUCOSES SOLUBLE IN ALCOHOL.

§ 70. Detection and Estimation of Glucoses soluble in Alcohol.Both glucoses and cane-sugars may be present in that part of the alcoholic extract $(\S 48)$ which is soluble in water, but the amount can be but small, since the material is macerated at the ordinary temperature. It must, however, be taken into account, in order to avoid error. If the alcoholic extract contain no tannin or bitter substance, the aqueous solution may be tested for glucose with Fehling's solution ( $(83)$ without further treatment; if found it may be determined quantitatively.

Sugar may also be qualitatively tested for by adding to the liquid under examination first potash and then dilute solution of sulphate of copper, as long as the cupric hydrate first formed is redissolved. Excess should be avoided. The liquid may now be divided into two portions, one of which may be warmed and the other allowed to stand in the cold in order to ascertain whether reduction takes place at the ordinary temperature, as well as on heating.

If the glucose is accompanied by such substances as tannin, etc., the filtrate obtairued after addition of acetate of lead in their quantitative estimation ( $\S \S 49,52)$, or after precipitation of a separate quantity with basic acetate, may be treated, together with the washings, with a slight excess of sulphuric acid, filtered, washed and made up to a known volume. The sugar may then be estimated quantitatively with Fehling's solution. The result must be added to the amount found in the aqueous extract (\$ 83). Part of the solution may be boiled for half an hour with 1 to 2 per cent. of sulphuric or hydrochloric acid in a flask fitted with an upright condenser. . If more sugar be found after such treatment, the difference is to be calculated as saccharose ( $\$ 85)$. 


\section{V.}

\section{Examination of Substances Soluble in Water.}

MUCILAGE, ACIDS, GLUCOSES, SACCHAROSES AND OTHER CARBOHYDRATES, ALBUMINOUS SUBSTANCES, ETC.

\$ 71. Treatment with Water:-The residue of the material after exhaustion with alcohol $(\S 47)$ is dried at a temperature not above $40^{\circ} \mathrm{C}$., and transferred to the vessel previously used, which should likewise be dried. Water is then added in the proportion of at least $10 \mathrm{cc}$. for every gram of original substance, and the whole frequently shaken during twenty-four hours. The liquid is now filtered off through the same filter that has already served for such operations, and the filtrate set aside for examination. The residue is washed by repeated maceration and filtration, the washings being reserved for treatment as directed in $\S 194$. The insoluble substance is not dried (\$S 92, 102, 105 et seq.; 193 et seq.).

\$72. Total Solid Residue. - $10 \mathrm{cc}$. of the filtrate are evaporated in a tared platinum dish, dried at $110^{\circ}$ and weighed. The residue is then incinerated and the ash deducted. It should be ascertained if the ash is rich in carbonic, sulphuric, or phosphoric acid, chlorine, lime, magnesia or potash ; and if large quantities of sulphuric or phosphoric acid are present they should be estimated $(\S 82)$.

If the filtrate contain much sugar, moisture may easily be retained by the residue. In such cases Serrurier ${ }^{1}$ advises the addition of $\frac{1}{2}$ per cent. of alcohol before evaporation. 'It is claimed that the residue is then porous and easily dried.

EXAMINATION OF MUCILAGINOUS SUBSTANCES, DEXTRIN AND ALLIED CARBOHYDRATES PRECIPITATED BY ALCOHOL.

\$ 73. Mucilaginous Substances. -10 to 20 cc. of the aqueous extract (\$71) are mixed with two volumes of absolute alcohol, and

1 Zeitschr, f. anal. Chemie, x. 491. 
allowed to stand for twenty-four hours in a cool place in a wellclosed vessel. The precipitate is collected on a tared filter, washed with 66 per cent. spirit, dried and weighed. Both filter and substance are then incinerated and the ash weighed, that of the filter being deducted. If the precipitate itself possess the characters of vegetable mucilage $(\$ \$ 195,196)$ and contain not more than 5 per cent. of ash, it may be assumed the latter corresponds to the lime and potash usually found in such mucilages. But if the percentage of ash be larger, and it contain much carbonate of lime or potash, attention should be paid to the possible presence of salts of vegetable acids with these bases, such as acid tartrate of lime or potash, etc. ( $\$ 74)$.

That the precipitate really contains vegetable mucilage may be proved by its dissolving in water to a mucilaginous liquid which does not reduce Fehling's solution until after it has been boiled for some time with dilute hydrochloric acid. Its concentrated solution is precipitated by basic acetate of lead. It is also occasionally precipitated by ferric chloride and thickened by solution of borax or soluble silicate of soda. See also $\$ 193$ to 196.

§ 74. Vegetable Albumen.-Incomplete solubility of the mucilage precipitate would indicate the presence of albumen, but, by the method of examination adopted, the quantity will usually be so small that it may be neglected. (See also \$ 92 et seq.; 95 et seq.) If, however, Lassaigne's test show that the precipitate contains much nitrogen, the results of the estimation of legumin and albumen, which will be subsequently made, must be deducted from the weight of mucilage, etc. If, on treating the mucilage precipitate with a little water, a difficultly soluble crystalline substance be observed, examination should be made for tartrate of lime or acid tartrate of potash, which, if present, should be estimated by precipitating with neutral acetate of lead and should be deducted from the weight of the mucilage.

$\S 75$. Inulin.-If subterranean parts of plants belonging to Compositæ or allied orders are under examination, they may, even though previously dried, yield a little inulin to water. After precipitation with alcohol it is not redissolved by water at the ordinary temperature, but is freely soluble when warmed to $56^{\circ}$. It is lævo-rotatory, is converted by treatment with dilute acid into levulose, and may be estimated by determining the 
amount of sugar thus produced. The majority of the inulin is, however, left in the residue insoluble in water, from which it may be extracted as directed in $\S 102$.

\$76. Dextrin, etc.-The filtrate and wash-alcohol from the mucilage precipitate $(\$ 73)$ are evaporated as rapidly as possible, at a temperature of $70^{\circ}$ to $80^{\circ}$, to a syrupy consistence and again precipitated with 4 volumes of absolute alcohol. Certain carbohydrates soluble in dilute alcohol, such as dextrin, levulin, sinistrin and triticin, are thus thrown down and should be filtered off as rapidly as possible.

These carbohydrates may be distinguished from mucilage by their being more easily convertible into sugar and by their not being precipitated by basic acetate of lead. Dextrin is dextrorotatory in aqueous solution, and yields grape sugar on boiling with a dilute acid. Levulin, sinistrin, and triticin yield levulose. The first of these three is optically inactive; sinistrin and triticin are lævo-rotatory $\left(\alpha_{\mathrm{D}}=-32.456^{\circ}\right.$ and $-43.579^{\circ}$ respectively). None of the four are coloured either blue or red by iodine. ${ }^{1}$ Sinistrin and triticin are precipitated by caustic baryta from solution in 40 per cent. alcohol. Carbonic acid liberates the carbohydrate from the ${ }^{\circ}$ compound thus produced (\$198).

Quantitative Estimation (\$S 199, 201 to 204). -The carbor hydrates mentioned in the preceding paragraph are best estimated by boiling with a dilute acid and determining the amount of sugar thus produced by titrating with Fehling's solution. The barium precipitates of levulin, triticin and sinistrin may be treated directly with acid.

If dextrin and glucose are present together, the results yielded by the estimation are as a rule somewhat too high, as a little. sugar is precipitated with the dextrin.

It should, however, be ascertained whether the dextrin-precipitate contain much nitrogen, and, if this is the case, whether the amido-acids discussed in $\$ 101$ and 242 are present.

\section{EXAMINATION FOR SAPONIN AND ALLIED SUBSTANCES.}

\$77. Extraction of Saponin.-If the precipitate obtained with alcohol in $\S 76$ is rapidly filtered off, the majority of the saponin

${ }^{1}$ It was formerly thought that dextrin was coloured red by iodine. This colouration was due to an impurity (soluble starch-erythrodextrin) contained in the dextrin examined. 
remains in solution, and is left behind on evaporating the alcoholic filtrate. It is soluble in hot 83 per cent. spirit and deposited again on cooling; but in absolute alcohol it is almost insoluble. Baryta-water precipitates it from aqueous solution; after washing with saturated baryta-water the saponin may be liberated from the compound by carbonic acid gas ; a few per cent. of baryta, however, always remain associated with the saponin thus obtained. It also forms an insoluble compound with basic acetate of lead. Its solutions have an unpleasant acrid taste, froth on shaking, emulsify oils, etc. On agitating with chloroform it is taken up by that solvent and may be obtained in an amorphous condition by evaporating the chloroformic solution. (Cf. §55.) The residue, moistened with a few drops of concentrated sulphuric acid and exposed to the air, gradually assumes a reddish or reddish-violet colouration. It is a glucoside, yielding sapogenin as a resinous decomposition product sparingly soluble in water.

§ 78. Quantitative Estimation.-Christophsohn ${ }^{1}$ and Otten $^{2}$ have adopted the following two methods for the determination of saponin :

A. 10 grams of the powdered substance are boiled three times in succession with distilled water, the decoctions strained '(they filter very slowly), evaporated to a small bulk, precipitated with alcohol and filtered. The precipitate is exhausted with boiling alcohol (83 per cent.), and the spirituous solution added to the filtrate. After recovering the alcohol by distillation the residue is dissolved in water, concentrated and precipitated with saturated baryta-water. The precipitate is collected on a tared filter, washed with saturated baryta-water till the washings are colourless and dried first at $100^{\circ}$, subsequently at $110^{\circ}$. After weighing it is ignited till the ash is white, the baryta estimated as carbonate in the usual way, calculated into oxide and deducted from the weight of the saponin-baryta, the difference being the weight of saponin from 10 grams of substance. For the seeds of Agrostemma githago the following modification must be adopted on account of the large amount of starch rendering the extraction with water very tedious. A weighed quantity of ground air-dry seecls are

1 'Vergl. Unters. über das Saponin, etc.' Diss. Dorpat, 1874, and Archiv d. Pharm. vi. $432,481$.

${ }^{2}$ Histiol. Unters. der Sarsaparillen. Diss. Dorpat, 1876. 
exhausted by boiling with alcohol and filtering whilst hot, the alcohol being recovered from the filtrate by distillation. The residue is freed from fatty oil by ether, dissolved in water and the saponin in it precipitated with baryta as before.

$B$. The saponin-baryta obtained by the previous method is dissolved in water with the aid of hydrochloric acid and freed from baryta by the cautious addition of dilute sulphuric acid. The filtrate and washings from the sulphate of barium are boiled for an hour; the sapogenin which has separated out is filtered off, washed, transferred together with the filter to a small flask and exhausted by boiling with 83 per cent. of alcohol. On evaporating the filtered alcoholic solution and drying at $110^{\circ}$ the weight of the sapogenin is ascertained and may be calculated to saponin, 100 parts of the latter yielding on an average 35.8 parts of the former.

Christophsohn obtained the following results in a series of comparative experiments with both methods. The seeds of Agrostemma githago were treated as directed in $A$.

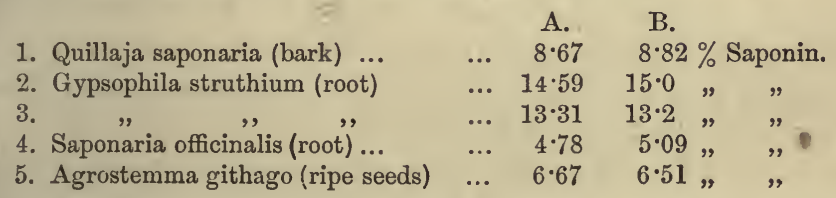

In the various sarsaparillas Otten found, by method $A$, from 1.21 to 3.43 per cent. of saponin. (See also $\S 167$.)

$\S$ 79. Digitonin, which is allied to saponin, may be distinguished by its assuming a fine red colour when heated with dilute sulphuric or hydrochloric acid. Like saponin, it is easily soluble in cold water, sparingly in cold absolute alcohol. (Cf. $\S 155,167$.

EXAMINATION FOR ACIDS, ETC.

\$ 80. Estimation of Total Organic Acids.-Part of the filtrate obtained in $\$ \$ 73$ and 76 is concentrated and, after complete dissipation of the alcohol, precipitated with neutral acetate of lead, avoiding an excess. After standing from twenty-four to forty-eight hours the precipitate is filtered off and treated as directed in $\S 49$. The organic matter present is noted as organic acids and allied substances. If the presence of tannic acid, which has escaped removal by the previous treatment with alcohol, is suspected, it 
should be estimated in a portion of the filtrate from $\S 73$ or $\S 76$, by precipitation with acetate of copper (cf. $\S 50$ ), and deducted from the total organic acids.

$\S 81$. Qualitative Separation.-If the lead precipitate is at first amorphous, but becomes crystalline on standing, malic or fumaric acid $^{1}$ may be present. (See also $\$ 214,220,221$.)

The acids thus precipitated may befurther qualitatively examined by suspending the moist precipitate obtained as directed in $\$ 80$ in pure water and decomposing with sulphuretted hydrogen. The filtrate from the sulphide of lead is evaporated on the water-bath to a small bulk and, when the odour of sulphuretted hydrogen has disappeared, lime-water is added to the cooled liquid till the reaction is alkaline. If a precipitate is produced which dilute acetic acid fails to dissolve completely, oxalic acid is probably present. $^{2}$ (See also $\S \S 214,218,219$.) If, on the other hand, it is entirely: soluble in acetic acid, a fresh portion should be tried with solution of chloride of ammonium. Tartrate of calcium ( $\$ 217$ ) dissolves, racemate $(\$ 218)$ does not. In the latter case care should be taken not to mistake phosphate for racemate of calcium.

If lime-water has caused no precipitate in the cold the solution should be boiled. Any turbidity that may now occur would indicate citric acid. $\quad(\$ \$ 215,216,218$.

Aconitic acid is not thrown down by lime-water even on boiling, but it is characterized by the slight solubility of its acid ammonium salt in 50 per cent. alcohol. The liquid to be tested is divided into two portions, one of which is neutralized with ammonia and added to the other. Any crystals of acid aconitate of ammonium which separate out should be washed with 50 per cent. alcohol. From this salt the acid may be isolated by adding a slight excess of sulphuric acid and shaking with ether. Its identity may be established by the ultimate analysis of the calcium, silver and ammonium salts. (See also $§ 216$.)

I think it is very probable that the so-called Marattin is aconitate of calcium (\$102). Sphæro-crystals of this substance were

1 For the solubility of malate of lead in warm dilute acetic acid, and the deposition of a crystalline salt on cooling, see Hartsen, Zeitschr. f. anal. Chemie, xiv. 373 (Journ. Chem. Soc. xxix. 375).

2 Oxalate of calcium ( $\$ \S 100,219)$ often settles slowly and on filtration passes through the pores of the filter. Muck has shown (Zeitschr. f. anal. Chemie, ix. 451 ) that the precipitate is much easier to manipulate if small quantities of aluminium salts are present. 
observed by Russow in the stems of species of Marattia which had been kept in alcohol.

If the presence of an oxalate has been indicated by the action of acetic acid, the acid solution should be filtered off and supersaturated with lime-water, which would re-precipitate tartaric, citric, racemic acid, etc. Citric acid may be detected by boiling the filtrate. Citric and tartaric acids may be separated quantitatively by Allen's method. ${ }^{1}$ The acids are dissolved in 20 volumes of spirit, and, a concentrated solution of acetate of potassium added. After standing twelve hours the acid tartrate of potassium is collected and estimated either gravimetrically, or by titration with normal solution of soda. (See also $\$ \$ 214$ et seq ; 217 et seq.)

§ 82. Vol. Estimation; Mineral Acids; Free and Combined Acid. - If only one of the non-volatile acids mentioned can be detected, the estimation made in $\S 80$ may be checked by decomposing the lead precipitate from a known fraction of the aqueous extract with sulphuretted hydrogen, evaporating the filtrate and titrating the residue dissolved in water. But in this case the phosphoric and sulphuric acids previously estimated in $\$ 7$ must be deducted (\$214).

Mineral acids may be tested for qualitatively by adding a drop of an alcoholic solution of methyl-violet. Mineral acids change the colour to bluish-green.

The amount of free acid present in fruits, etc., may be estimated in the aqueous extract by titration with normal alkali. A similar determination may be made in the alcoholic extract (\$ 47). Any excess of acid found in the first estimation over that in the second may generally be ascribed to the acid salts present.

If an extract from a vegetable substance is to be specially examined for free tartaric acid in the presence of acid tartrates (of calcium and potassium), the liquid may be evaporated to a syrupy consistence and the tartaric acid extracted with ether or absolute alcohol. ${ }^{2}$ The alcoholic or ethereal solution is evaporated, the residue dissolved in a little spirit and the tartaric acid separated by the addition of alcoholic solution of acetate of potassium. ${ }^{3}$

${ }^{1}$ Zeitschr. f. anal. Chemie, xvi. 251 (Pharm. Journ. Trans. [3], vi. 6).

${ }^{2}$ Cf. Claus, Zeitschr. f. anal. Chemie, xvii. 314.

${ }^{3}$ See also Nessler, Zeitschr. f. anal. Chemie, xviii. 230 (Journ. Chem. Soc. xxxvi. 981). 
EXAMINATION FOR GLUCOSES, SACCHAROSES, ETC.

\$83. Glucoses. - The alcoholic extract may, as mentioned in $\$ 70$, contain a small amount of glucose which, if present, should be quantitatively estimated. But, as was there observed, the whole of the glucose is not usually removed by cold alcohol, and the remainder must be looked for in the aqueous extract. If no tannic acid or other substance that reduces Fehling's solution is present, the glucose may be estimated in part of the aqueous extract in $\S 71$ by direct titration. ${ }^{1}$ But if the glucose is accompanied by other substances that also reduce the salt, these must be removed before the estimation can be made. They may be avoided by using the filtrate from the mucilage precipitate ( $(73)$, or from the dextrine group ( $\$ 76)$; the alcohol must be removed by evaporation, the residue dissolved in water and made up to a known volume. (Cf. § 197.) If such substances as tannic acid, etc., have to be removed, it is best to precipitate a portion of the aqueous extract with basic acetate of lead and remove the excess of lead with sulphuric acid before determining the sugar.

Instead of keeping the alkaline copper solution recommended by Fehling ready for use, I keep separate solutions of the three salts of which it is composed, viz., 34.639 grams of crystallized sulphate of copper, 173 grams of Rochelle salt, and 120 grams of caustic soda, each in a litre of water. $10 \mathrm{cc}$. of each of these solutions with $20 \mathrm{cc}$. of water represents $10 \mathrm{cc}$. of alkaline copper solution diluted with 4 volumes of water as recommended by Fehling. Of the three solutions the sulphate of copper alone requires to be accurately measured.

The titration is made as follows: the alkaline copper solution is brought to the boil in a white porcelain dish and the sugar solution (previously made up to a known volume) added from a burette until the blue colour has completely disappeared. $10 \mathrm{cc}$. of copper solution indicate 0.05 gram grape-sugar. Should the final disappearance of the blue colour be concealed by dark colouring matter, etc., in the solution, a few drops may be rapidly

1 On the estimation of sugar with copper solution, see Fehling, Annalen d. Chem: u. Pharm. Ixxii. 106, cvi. 75 (Pharm. Journ. Trans. [1], ix. 419); Graeger, N. Jahr. f. Pharm. xxix. 193 ; O. Schmidt, ib. 270; Stædeler u. Krause, Annalen d. Chem. u. Pharm. lxix. 94 ; Pellet, Journ. de Pharm. et de Chimie, 4te Série, xxvii. 460 (Journ. Chem. Soc. xxxiv. 612). 
filtered off and tested for copper by the addition of acetic acid and ferrocyanide of potassium. But a slight reaction will generally be obtained as traces of copper remain in solution and the absence of any reddish-brown precipitate after the lapse of a few minutes must be taken as sufficient indication of the termination of the reaction.

It is well known that the sugar solution should be very dilute; the best strength is about $\frac{1}{2}$ per cent. If a preliminary experiment shows it to be more concentrated, it should be diluted to about this strength. ${ }^{1}$

The estimation may also be made gravimetrically, by quickly filtering off the cuprous oxide, washing with water, drying and converting into cupric oxide. This method is advisable if, in titrating, the final reaction is obscured, or if the amount of sugar solution available is not sufficient to complete the reduction of the copper-salt taken.

But it must be borne in mind that by drying the cuprous oxide on the filter and weighing incorrect results would, as Brunner ${ }^{2}$.has shown, be obtained, since the alkaline copper solution dissolves cellulose and the filter accordingly loses weight. It is better, therefore, to dissolve the cuprous oxide and determine the copper by the usual methods, or to estimate the excess of copper in the filtrate. ${ }^{3} 317$ parts by weight of copper, 357 of cuprous oxide or 397 of cupric oxide indicate 180 of glucose, 171 of saccharose or 162 of starch, etc. ( $\$ 200)$.

\$84. Other Methods of Estimating Glucose.-Glucose may also be estimated by Knapp's reagent, ${ }^{4}$ which consists of 10 grams of

${ }^{1}$ Soxhlet-Zeitschr. f. anal. Chemie, xviii. 348 (Pharm. Journ. Trans. [3] xi. 720)-has shown that the reducing power of the glucose varies with the concentration of the solutions. In making estimations the sugar solution should therefore be of as nearly as possible the same strength as that used for standardizing. According to Soxhlet the gravimetric estimation in presence of an excess of copper may be attended with considerable error. But Maercker has shown that satisfactory results may be obtained by this method also, if the same conditions are always observed. See also Ulbricht, Chem. Centralblatt, $1878,392,584$.

${ }^{2}$ Zeitschr, f. anal. Chemie, xi. 32 (Journ. Chem. Soc. xxv. 928).

${ }^{3}$ Compare also Weil, ib. 284 ; Mohr, ib. xii. 296 ; Jean, ib. 111 ; Lagrange, ib. xv. 111 ; Brücke, ib. 100 ; Maschke, ib. xvi. 425. (See Journ. Chem. Soc. xxv. 1121 ; xxvii. 292 ; xxxi. 805 , ib. 116 ; xxxii. 930.)

${ }^{4}$ Annal. d. Chem. u. Pharm. cliv. 252 (Pharm. Journ. Trans. [3] i. 301). See also Mertens, Zeitschr. f. anal. Chemie, xiii. 76 ; Brumme, ib. xvi. 121. Knapp's reagent keeps considerably better than Fehling's. 
mercuric cyanide and 100 cc. of caustic soda (sp. gr. 1·145) in a litre of water; 0.4 gram of the cyanide $=40 \mathrm{cc}$. of solution indicate 0.10 gram glucose. Knapp determines the end of the experiment by touching a drop of the solution on filter paper with a drop of sulphide of ammonium. An excess of mercury would produce a brown colour ( $(200)$.

Instead of Knapp's solution an alkaline solution of potassiomercuric iodide may be employed for estimating glucose, as recommended by Sachsse. The sugar solution for this reagent may be prepared as directed in $\S 83$. The reagent as first recommended by Sachsse $^{1}$ contained a large excess of alkali, which rendered the estimation of dextrose and levulose in the presence of saccharose inaccurate. Heinrich ${ }^{2}$ therefore altered the composition by reducing the amount of alkali to a minimum, and directed that a litre should contain 18 grams of mercuric chloride, 25 of iodide of potassium and 10 of caustic potash. 40 cc. indicate 0.1342 gram glucose. The titration is made in the same way as with Fehling's solution, and the end of the experiment determined by testing a drop with stannous chloride, which should not throw down a grey precipitate, showing that no excess of mercury remains in solution. The presence of ammonium salts does not interfere with the reaction. Nessler's reagent for ammonia has a composition similar to Heinrich's modification of Sachsse's solution, but contains a far larger quantity of caustic alkali, which is necessary for the detection of ammonia ( $\$ 97)$.

The appearance of the final reaction is retarded if the solution contains but very small quantities of invert-sugar. It is advisable to make the mercurial solution of such strength that $5 \mathrm{cc}$. indicate 0.0168 gram of invert-sugar.

Glucose may also be estimated gravimetrically by using an acid solution of a mercuric salt. 'ihe reagent recommended contains in a litre 30 grams of mercuric oxide, 25 of concentrated acetic acid and 30 of chloride of sodium. On boiling with sugar the mercury is reduced and may be weighed as mercurous chloride. ${ }^{3}$ $5 \cdot 88$ parts of calomel indicate 1 part of glucose.

1 Jahresb. f. Pharm. 1876, 375 (Journ. Chem. Soc. xxxii. 226). See also Strohmer u. Klauss, Chem. Centralblatt, 1877, 697, 713 (Journ. Chem. Soc. xxxiv. 246).

${ }^{2}$ Chem. Centralblatt, 1878, 409 (Journ. Chem. Soc. xxxvi. 180).

3 Jahresb. f. Pharm., 1877, 340. 
The reagent is said to be without action on cane-sugar, glycerin, arabin and dextrin.

§ 85.-Influence exerted by Saccharoses.-If the glucose in the liquid under examination is not accompanied by saccharose, or other carbohydrate not precipitable by alcohol, fairly accurate results may be obtained by the methods detailed in $\S \S 83,84$. But saccharoses influence the estimation by their presence to an appreciable extent, although they do not themselves, when pure, reduce Fehling's or Sachsse's solution.

The same applies to the determination by fermentation (\$204); saccharoses may be partially converted by the yeast into fermentable glucose.

It cannot be said that we are in a position to estimate with exactness in every case the proportion of glucose and saccharose in mixtures. Sometimes, it is true, the accuracy of the estimation leaves little to be desired-as, for example, mixtures of dextrose or invert-sugar with cane-sugar. Solutions of such mixtures may be examined in the polariscope, in addition to being tested chemically. But many instances occur in which the necessary conditions do not obtain. (Cf. $\S \S 208,209$.)

$\S 86$. Estimation in Presence of Saccharose.-In such cases the only method we can adopt is, first, to remove the carbohydrates precipitable by alcohol ( $\S 73,76$ ), estimate the glucose with Fehling's solution, and then repeat the estimation after acidifying with 1 per cent. hydrochloric acid and boiling for 15 to 20 minutes (or several hours if the presence of mycose be suspected) in a flask provided with an upright condenser. If the two determinations yield fairly concordant results, it may be assumed that no saccharose is present; on the other hand, any excess that the second may indicate over the first may be noted as 'saccharose or allied carbohydrate.' The possibility of error must, however, be admitted ( $\$ 207)$.

§87. Estimation of Saccharose alone.-If the solution contains a saccharose alone, with the exception of milk-sugar or maltose, it will not reduce Fehling at all. Although, therefore, no reduction may be observed, the inversion with acid should not on any account be omitted, as the solution may contain a saccharose. (Cf. § 207.) According to Pillitz, ${ }^{1}$ cane-sugar may be easily inverted by

${ }^{1}$ Zeitschr. f. anal. Chemie, x. 456 (Journ. Chem. Soc. xxv. 329). See also Nicol, ib. xiv. 177 (Journ. Chem. Soc. xxv. 329). 
heating a solution of 1 part in 12 or 13 of water with 1.5 to 2.0 parts per mille of sulphuric acid (specific gravity $1 \cdot 12$ ), in sealed tubes, to $130^{\circ}$ or $135^{\circ}$. The estimation of sugar by the fermentation of such solutions is said to yield numbers that are rather too low. That is not the case with determinations by Fehling's or Knapp's method.

I am, on the whole, more inclined to use hydrochloric acid for inverting; but if the acid is to be subsequently removed, I must acknowledge that sulphuric is to be preferred, as it is easily precipitated by carbonate of barium.

$\S 88$. Böttger's Test.-The above tests also suffice for the detection of glucose and saccharose. Böttger's bismuth test may be employed as confirmatory of the presence of the former. It consists in warming the liquid with a solution of carbonate of soda, together with oxynitrate or hydrate of bismuth ; if sugar be present, grey suboxide of bismuth is formed. (See also $§ 200$.)

\$ 89. Distinctive Characteristics.-The chief marks of distinction between the various members of the glucose or of the saccharose group are to be found in the difference in crystalline form, etc., and in the action on polarized light. In the cases here alluded to, use may sometimes be made of these characters, especially if the solution contains only one carbohydrate and no other substance that might influence the crystallization or optical activity. But these conditions are seldom fulfilled, and in the majority of cases we must, therefore, forego an exact identification of the particular glucose and saccharose present, unless we have a considerable quantity of the substance under examination at our disposal. (Cf. $§ \S 205-207$.$) •$

If we have command of a large quantity of material, it would be best to endeavour to effect the separation of the carbohydrates by treatment with different solvents, decolourization with animal charcoal and crystallization. The crystallization of glucose is favoured by direct sunlight; the presence of a small quantity of a mineral (hydrochloric) acid may also prove advantageous. (See also $§ \S 205-207$.)

§90. Soluble Modification of Arabic Acid. Albuminoids not Precipitated by Alcohol.-In almost every plant-analysis the sum-total of the separate estimations of the substances soluble in water (mucilage, etc.) will be found lower than the estimation of the total solids in solution. One or more substances must, therefore, 
generally be present that are soluble in water, not precipitated by alcohol or neutral acetate of lead and have up to the present time eluded investigation. It might appear hazardous to make conjectures as to the nature of these substances, but I cannot help remarking that in some cases a substance seemed to me to be present which, after evaporation of its alcoholic or aqueous solution, did not again dissolve completely in either of those liquids. It appeared to agree in some of its properties with that form of vegetable mucilage that is obtained by dialyzing acidified solutions of gum, etc., which sometimes remains in solution on the addition of alcohol. When I have met with a substance agreeing with mucilage in this peculiarity, I have spoken, it is true, of a 'soluble modification of arabic acid,' but I have not omitted to place a query after it. ${ }^{1}$ The further investigation of this substance is a desideratum for plant-analysis.

But in thus assuming the presence of such an 'arabic acid,' account must be taken of the results of the nitrogen determinations to be described in $\S 96$. By deducting the nitrogen in the residue of the material after extraction with water from that in the original substance, the amount in the sulstances soluble in water is ascertained. If, now, the amount of nitrogen present as albuminoids, nitric acid, ammonia and alkaloid is calculated from the separate determinations and found to be much smaller than the estimation by difference, it should be remembered that under certain conditions water may dissolve albuminoids which alcohol fails to precipitate.

\$91. Mannite.-Another substance, however, which is of not unfrequent occurrence in the vegetable kingdom, would similarly elude detection by the foregoing experiments with the alcoholic and aqueous extracts, as it is almost insoluble in cold absolute alcohol but is not precipitated from its aqueous solution by the addition of either spirit or lead salts. The substance referred to is mannite. If present it would be included in the deficit mentioned in $\$ 90$, but would be easy of detection, as it crystallizes with great facility in long prisms and needles and is somewhat sparingly soluble in cold spirit. It may be approximately estimated by precipitating the aqueous solution with alcohol and basic acetate of lead,

${ }^{1}$ Compare rny 'Chem. Beiträge z. Pomologie,' Dorpat, 1878 ; Verlag d. Dorpater Naturforscher Gesellsch. ; and Pfeil, 'Chem. Beiträge z. Pomologie,' Diss. Dorpat, 1850. 
removing the lead by sulphuretted hydrogen and any glucose that may be present by rapid fermentation. The residue may be exhausted with boiling 90 per cent. alcohol and allowed to crystallize in the cold. But an accurate result cannot be expected, since, in addition to other errors, mannite may be produced in considerable quantity by the fermentation of cane-sugar. ${ }^{1}$ For particulars of some substances allied to mannite see $\$ 212$.

The method of examination for bitter principles, glucosides, and alkaloids has been described in $\$ 58$ to 69 . (See also $§ \S 165$ et seq. ; 171.)

\section{EXAMINATION FOR ALBUMINOIDS SOLUBLE IN WATER,} AMMONIACAL SALTS AND NITRIC ACID.

\$ 92. Extraction of Albuminoids.-It has already been observed in $\$ 74$ that if the residue, after extraction with ether and alcohol, be exhausted with water the estimation of albuminoids in the aqueous extract thus prepared will generally give inaccurate results. A fresh portion of material should therefore be directly exhausted with water, or, if much fixed oil is present, the extraction with water may be preceded by treatment with petroleum spirit. After having removed the fixed oil (if necessary) from about 10 grams, the residue is dried at $40^{\circ} \mathrm{C}$., macerated with $100 \mathrm{cc}$. of water, with frequent agitation, for 4 to 6 hours, and filtered as described in $\$ 71$. If thought desirable the maceration may be conducted at a temperature not exceeding $35^{\circ}$ to $40^{\circ}$. (Compare also $\$ 2: 5$ et seq.)

Detection.-With a portion of the filtrate qualitative experiments should be made. Among the reagents used for the detection of albumen, iodine and mercuric nitrate (containing as little free nitric acid as possible) may be mentioned; the former colours it brown, whilst the latter produces a yellow colour, changing, on the addition of a trace of nitrous acid, to a splendid red. The addition of caustic potash to albumen, previously moistened with solution of sulphate of copper, is followed by the appearance of a bluish-violet colour. If the amount of albumen present be rather small, these experiments may be made with the precipitates obtained by the addition of an acid to the aqueous solution ( $\$ 93)$.

Microchemical.-These reagents also serve for the microchemical detection of albumen. The latter substance possesses, moreover,

${ }^{1}$ Archiv d. Pharm. xv, 47 (Journ. Chem. Soc. xxxviii. 100). 
the property of absorbing aniline-violet (protoplasm generally assuming a bluish-violet, the cell-nucleus a reddish tint), carmine, cochineal, picro-carmine, etc. Note should also be taken of the form in which the albumen occurs, whether crystalline or amorphous, etc. (See also $§ 74,90,95,194$.)

Protoplasm is coagulated by absolute alcohol and by glycerine. It becomes clear with solution of caustic potash, cloudy with acetic acid. Nuclei are generally stained more deeply than protoplasm by aniline-violet, etc., and by iodine. They are coloured deep blue by a solution of hæmatoxylin $(1: 30)$ and alum $(1: 10)$; the former alone also produces the same effect if the section has been previously treated with picric acid and the excess of the latter completely removed (Schmitz). Crystalloids dissolve in dilute potash, ammonia, and acetic acid.

Precipitation.-Albuminous substances are precipitated by ferrocyanide of potassium and acetic acid, by aqueous solution of trichloracetic acid, and by solution of xanthogenate of potassium. The precipitate produced by the last reagent becomes flocculent on heating to $30^{\circ}$ (Zöller). (See also $\$$ 95, 231, 232.)

\$ 93. Estimation of Legumin and Globulin.-Part of the filtrate ( 25 to 50 cc.) is acidified with hydrochloric acid in the cold. By this means such substances as legumin are precipitated; they should be collected on a tared filter, washed first with water acidified with hydrochloric acid, then with 40 per cent. spirit, dried and weighed, deducting ash (\$225 et seq.). If hydrochloric acid has caused a precipitate, a fresh portion of the filtrate should be tested for globulin by saturating with carbonic acid. It should also be ascertained microscopically whether the precipitate (if any) is crystalline or amorphous. (Cf. $\$ 226,227$.)

\$ 94. Estimation of Albumen.-To the filtrate from the legumin (without the spirit-washings), 5 to $10 \mathrm{cc}$. of a concentrated solution of chloride of sodium are added, together with enough acetate of soda to remove all the hydrochloric acid, and the whole raised to the boiling-point. If flocks of albumen separate they must be collected, washed first with boiling water, afterwards with 40 per cent. spirit, dried and weighed, deducting ash.

In the absence of legumin $25 \mathrm{cc}$. of the aqueous extract may be mixed with $5 \mathrm{cc}$. of a concentrated solution of chloride of sodium and a few drops of acetic acid and treated as described in the foregoing paragraph. (See also $§ 230$.) 
§ 95. Estimation of Total Albumen. (a) Precipitation with Tannin.-Another portion (25 cc.) of the aqueous extract is mixed with half its volume of a concentrated solution of salt and a solution of tannin and acetic acid in dilute alcohol ( 20 grams tannin, 37.5 cc. glacial acetic acid, 400 cc. spirit made up to a litre with water) added as long as a precipitate is produced. This is then rapidly filtered off, washed with water and dried. The albumen contained in it may be determined by estimating the nitrogen and multiplying by 6.25 (see $§ 224$ ), or by extracting the tannin from the powdered precipitate by boiling with alcohol, collecting and weighing the residue. (Cf. § 229.)

This estimation of albumen should be compared with the previous estimations of legumin $(\$ 93)$ and albumen (\$94). If the determination by tannin yields a higher result, the difference may be taken to represent albuminous substances not precipitated by hydrochloric acid or by boiling with acetic acid. (Compare also the remarks on peptone in $\$ 232$.)

As already observed in $\S 51$, in working with substances containing a large quantity of tannin, the results obtained by proceeding as directed in $\$ \$ 92$, et seq., cannot be quite accurate, as part of the albuminous matter is retained by the tannin in the residue insoluble in water. This undissolved albumen may be determined as directed in $\$ 996,224$.

Amongst the substances which facilitate the solution of albumen we may include arabin. Günsberg ${ }^{1}$ has proved that albumen, of animal origin at least, is precipitated by gum from slightly acid solutions, but redissolved by an excess. Dextrin is said to differ from gum in not redissolving the precipitated albumen when added in excess.

\$96. Total Nitrogen.-It is advisable to determine the total nitrogen in the substance under examination before and after exhaustion with water; the difference represents the nitrogen in the substances removed by that menstruum. If from this difference the nitrogen contained in the albumen estimated according to $\$ 93$ to 95 is deducted, the remainder will be nitrogen that has been dissolved by water in the form of ammoniacal salts, amides, alkaloids, nitrates, etc. The following estimations should be made with the object of determining as far as possible in what state this remaining nitrogen exists.

${ }^{1}$ Journ. f. pract. Chem. 1xxxviii. 239. 
$\S 97$. Ammonia. ${ }^{1}-\mathrm{A}$ portion of the aqueous extract $(\S 92)$ is mixed with two volumes of alcohol and filtered. To the filtrate and washings calcined magnesia is added, and the ammonia distilled off into a receiver containing a measured quantity of normal sulphuric or hydrochloric acid, every precaution being taken to avoid loss of ammonia and spirting of the magnesia mixture into the receiver. The apparatus I use is represented in Fig. 2. The flask $A$ should not be more than half full of magnesia

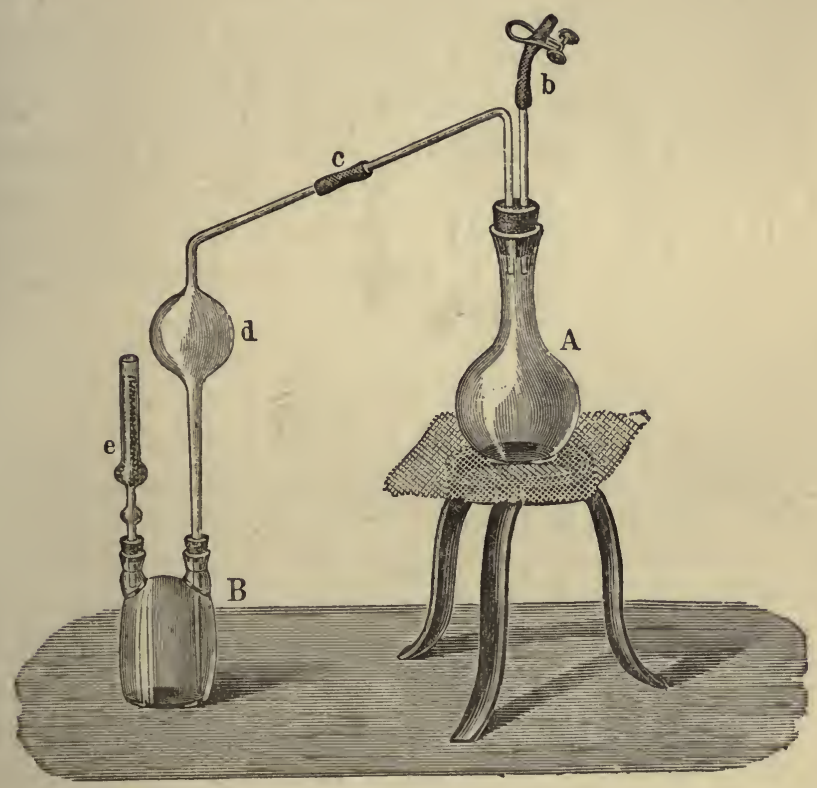

Fig. 2.

mixture, and a plug of glass wool should be inserted in the neck. The small tube $e$ contains glass beads, which are moistened with part of the acid. The distillation is complete when the vapours that issue on opening the clip $b$ are free from alkaline reaction. The estimation may be completed in either of the two following ways :

(a) The excess of acid in the receiver is determined volumetrically and deducted from the quantity taken. From the differ-

${ }^{1}$ Compare also Morgen, Zeitschr. f. anal. Chem. xx. 37 (1881). 
ence the amount of ammonia may be calculated in the usual way.

(b) The ammonia may be distilled into hydrochloric acid; the liquid evaporated to dryness, the residue alternately moistened and dried two or three times, and the chlorine estimated volumetrically by nitrate of silver and chromate of potash. From the chlorine found the amount of ammonia may be calculated.

Another method for the estimation of ammonia is that proposed by Schloessing. A few grams of the material made into a paste with water, or better, a concentrated aqueous extract, is mixed with milk of lime and placed over a measured quantity of volumetric sulphuric acid under a bell jar. The ammonia liberated by the lime is absorbed by the sulphuric acid, and after standing two or three days at a temperature as nearly constant as possible $\left(8^{\circ}\right.$ to $\left.10^{\circ}\right)$, the amount of acid thus neutralized may be ascertained by estimating the excess with volumetric solution of soda, and from this the ammonia may be calculated.

It must be admitted ${ }^{1}$ that in all these experiments the action of the lime or magnesia on albuminous substances may result in the formation of ammonia. It is advisable, therefore, to remove such substances by precipitation with basic acetate of lead before distilling. Glutamine and asparagine, however, remain in solution. These substances, when pure, are not acted upon by either lime or magnesia, but Schulze believes that they undergo a partial decomposition in mixtures, and therefore recommends boiling with hydrochloric acid for one to two hours (compare remarks on asparagine, $\S 191$ ), by which they are completely resolved into the corresponding amido-acids and chloride of ammonium. The estimation of ammonia now includes the total ammonia derived from the asparagine and glutamine. These two substances may, however, be determined by Sachsse's method, and the ammonia they yield calculated and deducted.

If the precautions mentioned have been observed, the first method $(a)$ will generally yield satisfactory results.

§98. Amido-Compounds, etc.-The foregoing estimation will be inaccurate if the material under examination contains amido-compounds, etc., or volatile alkaloids, as the former yield ammonia and the latter distil over and saturate part of the acid. Many amines,

1 Compare E. Schulze, Zeitschr. f. anal. Chem. xvii. 171 (1878); Journ. Chem. Soc. xxxiv. 308 . 
etc., thus liberated, yield with perchloride of platinum double salts (\$183) that are soluble in ether-alcohol, and error may therefore be frequently avoided by precipitating a second portion of the distillate with excess of perchloride of platinum, evaporating on the water-bath, extracting the residue with ether-alcohol, drying and weighing instead of titrating the excess of acid with an alkali. If both experiments yield the same result it may be concluded with tolerable certainty that no amides, or only traces, are present. If the estimation by the first method gives a higher result than that by the second, the former is to be regarded as the more accurate, and the excess noted as amido-compounds, etc. If, on the other hand, the estimation by platinum is higher than that by titration, the presence of an amide forming a double platinum salt insoluble in ether-alcohol and of a higher molecular weight than ammonia would be indicated. In the method of determining ammonia described in $\S 97, b$, certain chlorides of amines and alkaloidal substances, as for instance coniine and nicotine, would be almost completely volatilized, and thus escape estimation.

The separation of ammonia from many amines may frequently be effected by taking advantage of the difference in solubility of the chlorides, sulphates, and oxalates of the respective bases in alcohol. In preparing larger quantities of the base for closer investigation, the material might be distilled with magnesia or lime $(97 a)$, the distillate received in one of the above-mentioned acids, and evaporated to dryness on the water-bath. The residue might be extracted with alcohol, the solution again evaporated to dryness, and the distillation with alkali repeated, if possible, in a current of hydrogen. (Cf. § 239.)

$\S 99$. Nitric Acid.-For the estimation of nitric acid another portion of the aqueous extract of $\S 71$ is taken and treated by Schulze's ${ }^{1}$ or Wulfert's ${ }^{2}$ method.

Schulze directs the liquid to be treated first with pure potash, as long as ammonia is evolved, then with permanganate of potassium (free from nitrate) till the colour is permanent after ten minutes' boiling. Excess of permanganate is removed by formic acid, the solution neutralized with pure sulphuric acid and evaporated to about $10 \mathrm{cc}$. This is then introduced into the flask $A$ of the

1 Zeitschr. f. anal. Chem. vii. 392.

${ }^{2}$ Landw. Versuchsstationen, xii. 164. 
apparatus recommended by Schulze ${ }^{1}$ (Fig. 3), a weighed quantity of powdered aluminium added, and solution of caustic soda slowly run in; from the deficit in the amount of hydrogen yielded the

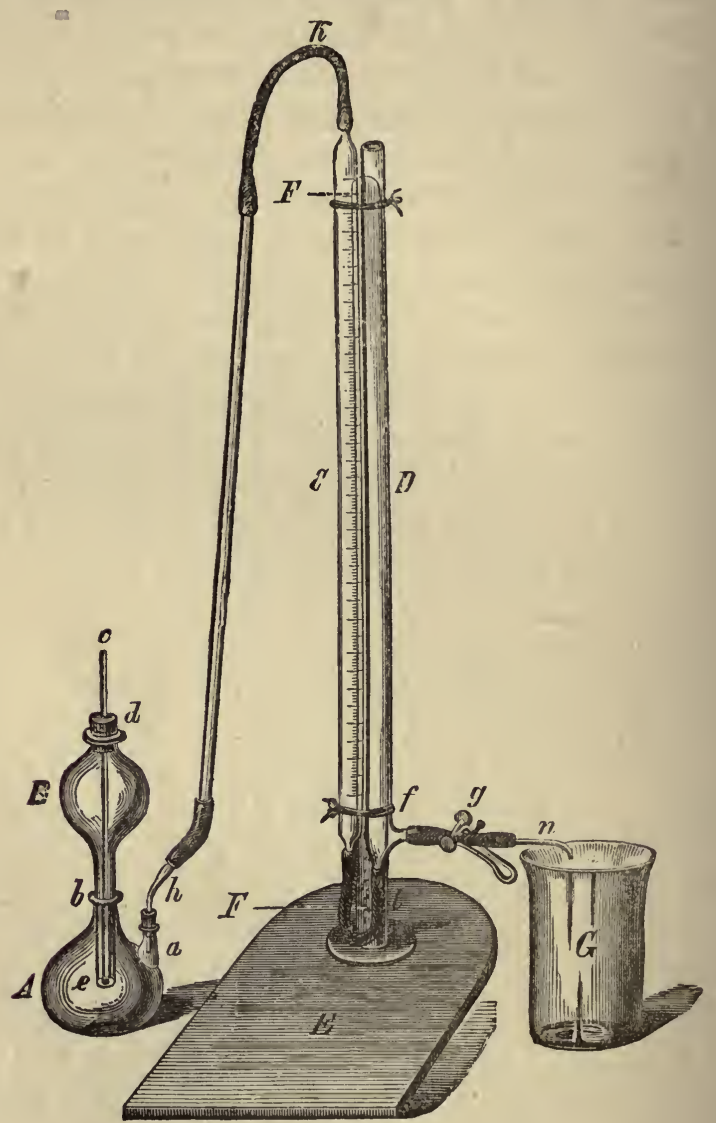

Fig. 3.

nitric acid present may be calculated. The following are the details of the operation :

A measured quantity of solution of caustic soda is introduced into the pear-shaped flask $B$. The end of the glass-rod $c$ is accurately ground into the delivery-tube of $B$, so that no soda can escape into $A$ until the rod $c$ is raised. The tube $C$ is

1 Zeitschr. f. anal. Chem. ii. 379, and vi. 379. 
graduated, and communicates with $D$ by means of an indiarubber tube. Both $C$ and $D$ are filled with water till the zero in $C$ is reached, the water standing at the same height in $D$. The solution of soda is then allowed to flow slowly into $A$ (which already contains the liquid and powdered aluminium), so that the experiment may last from two to three hours. The hydrogen evolved causes a rise of the water in $D$, but by occasionally opening the clip at $g$, it may be maintained at about the same level in both tubes. Care must be taken at the end of the experiment that the level is exactly the same before the final reading is taken. From the volume of gas thus found the volume of the caustic soda introduced from $B$ must be deducted, and the remainder corrected for temperature,

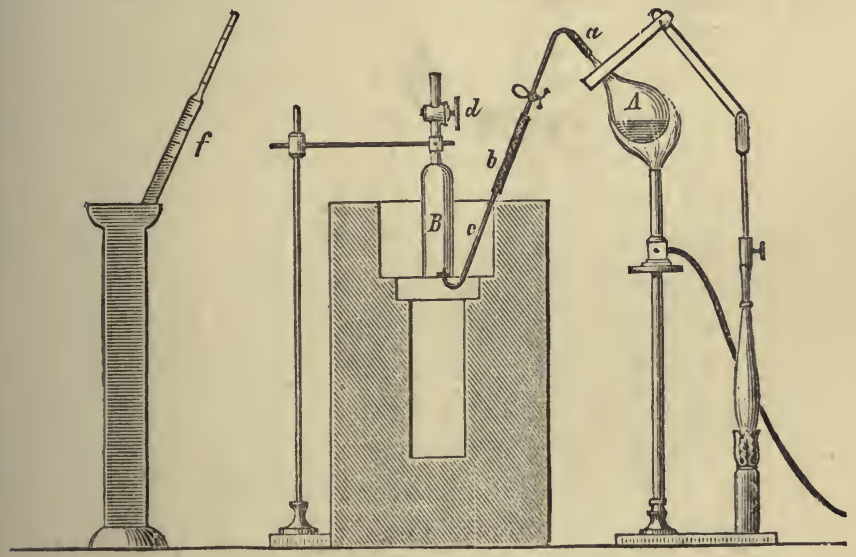

Fig. 4.

pressure, and tension of water-vapour. A previous blank experiment having shown the amount of hydrogen obtainable from the aluminium taken, the nitric acid may be calculated from the deficit, 4 molecules of hydrogen corresponding to 1 of nitric acid or nitrate of potassium.

$\S 100$. Wulfert's method is a modification of Schloessing's devised by Schulze : 0.5 to 1.0 gram of the powdered substance is boiled with water to which a little milk of lime has been added, filtered and washed; the filtrate and washings are then evaporated to 30 or 40 cc., and again filtered. The filtrate is neutralized with hydrochloric acid and introduced into the flask $A$ (Fig. 4), the neck of which has been drawn out so as to admit of connection 
by means of an indiarubber tube with the bent glass tube $a$. The longer leg of the latter is similarly connected with a second bentglass tube $c$, communication being regulated by a clip at $b$. The clip being opened, the atmospheric air in the flask is completely expelled by boiling the liquid down to at least one-fourth of its original bulk. The end of the glass tube $c$ is then introduced into a precipitating glass containing about $30 \mathrm{cc}$. of a concentrated solution of ferrous chloride, and, after allowing a little steam to escape, the clip at $b$ is closed and the lamp removed. As soon as a partial vacuum has been produced in $A$, the clip is cautiously opened, and about $20 \mathrm{cc}$. of the iron solution allowed to enter. The precipitating glass is then filled with hydrochloric acid (sp. gr. 1.12), and 25 to 40 cc. introduced in a similar manner, so as to sweep the iron solution out of the tubes into the flask. After closing the end of the tube $c$ with an indiarubber stopper, it is introduced into a mercury-bath and brought under a cylinder $B$, previously filled with mercury. The stopper is now removed, and the flask again heated until the pressure in the interior is nearly equal to that of the atmosphere. By opening the clip $b$, and regulating the pressure with the finger and thumb, the mercury is allowed to rise in the tube so as to drive most of the hydrochloric acid into the flask; it must, however, itself be carefully prevented from passing into the latter. After the external pressure has been overcome, the heat is so regulated that half the liquid in the flask distils over in eight to ten minutes. It is then certain that all the nitric oxide that has been formed has been driven into $B$. The latter is provided with a glass-tap $d$, and can be connected air-tight with a measuring tube $f$. After cooling, the measuring tube filled with mercury is fitted on to the cylinder, and the nitric oxide transferred to it by opening the tap and sinking the cylinder. The amount of nitric acid may then be calculated from the volume of nitric oxide found. ${ }^{1}$

$\S 101$. Sclerotic, Cathartic Acid, etc.-If the total amount of nitrogen in the aqueous extract $(\S 96)$ is now found, on comparison, to be larger than that present as albuminoids, alkaloids, ammonia and nitrates already estimated, the excess may be reasonably ascribed to certain albuminous substances not pre-

${ }^{1}$ On the estimation of nitric acid in cultivated plants, see also Schloessing, Journ. f. pract. Chem. lii. 142; Frühling und Grouven, Landwirthsch. Versuchsst. ix. 9, and 150 (1867); Reichardt, Zeitschr. f. anal. Chem. ix. 24 (1870) (Journ. Chem. Soc. xxiv. 439). 
cipitated in $\$ \S 93,94$, or certain amido-acids, such as sclerotic or cathartic acid, etc. (For the latter see $\$ 242$.)

\section{EXAMINATION FOR INULIN.}

\$102. Extraction and Estimation.-It has already been mentioned in $\S 75$ that in dried drugs the majority of this carbohydrate is present in the form of an insoluble modification; in fresh it is always dissolved in the cell-sap. Dried drugs may accordingly be treated first with cold water as directed in $\$ \S 71,92$, and the residue digested for some time with water at $55^{\circ}$ to $60^{\circ}$ (not higher). At this temperature inulin passes into solution. From a measured volume of the aqueous extract it may be precipitated by the addition of three volumes of alcohol; and if for every 100 cc. of mother liquor a correction of 0.1 gram of inulin is made, it may be thus estimated with tolerable accuracy. ${ }^{1}$

Characters.-Inulin is not precipitated in a gelatinous or curdy form, but in a pulverulent condition. It has already been observed that an aqueous solution is lævo-rotatory, and that boiling with a dilute acid converts it into lævo-rotatory fruit-sugar (levulose). Inulin may be satisfactorily estimated by converting it into levulose and titrating with Fehling's solution. Of course, the above mentioned correction must be made.

I should not, however, proceed to the extraction with water at $55^{\circ}$ to $60^{\circ}$ unless a preliminary experiment had indicated the probable presence of inulin.

Microscopical.-In dried drugs inulin usually appears, under the microscope, in the form of agglomerated masses in the parenchymatous cells. If fresh parts of plants that contain inulin are allowed to stand for several days in strong spirit, it is deposited in very characteristic sphæro-crystals, which dissolve in acid and alkali without swelling.

Inuloid, which is said to occur in spring in the rhizomes of plants of the natural order Compositæ, may also form similar sphæro-crystals, as do also marattin and a substance found in Acetabularia mediterranea which has not yet been closely investigated. (Cf. § 81.)

Inuloid is said to be distinguished from inulin by its somewhat greater solubility in water. ${ }^{2}$

\footnotetext{
${ }^{1}$ Compare my 'Materialien zu einer Monographie des Inulins.' St. Petersburg, 1870.

${ }^{2}$ Compare Annal. d. Chem. u. Pharm. clvi. 190.
} 
VI.

Examination of Substances Soluble in Dilute Caustic Soda; Metarabic Acid, Albuminous Substances, PhlobaPHENES, ETC.

$\S 103$. Extraction. - The residue insoluble in water ( $\$ 71)$ is suspended, whilst still moist, in water containing a known quantity (about 0.1 to 0.2 per cent.) $)^{1}$ of caustic soda in solution, using about 10 cc. of alkaline liquid for every gram of original substance. After standing for about twenty-four hours, with frequent agitation, the mixture is filtered. From $20 \mathrm{cc}$. to $50 \mathrm{cc}$. of the filtrate are acidified with acetic acid, mixed with 3 volumes of 90 per cent. alcohol, and allowed to stand for twenty-four hours in a cool place. The precipitate is then collected on a tared filter, washed with 75 per cent. alcohol, dried, and weighed, deducting ash. This precipitate usually consists of mucilaginous substances (pectin) and albuminoids. The former generally corresponds to Scheibler's metarabic acid ( $\$ 195)$.

$\S 104$. Detection and Estimation of Albumen.-If Lassaigne's test shows the presence of a considerable quantity of albuminous substances, these should be estimated and deducted. To this end another portion of the filtrate is precipitated as in $\S 103$, the nitrogen in the precipitate estimated and calculated into albuminoids (\$224). This amount is then deducted from the weight of the precipitate in $\$ 103$. (See also $\$ 226$ et seq; 236 to 238.)

$\S 105$. Estimation.-But the amount of albuminous substances insoluble in water thus found cannot be noted as such in the summary of results unless it corresponds to that calculated from the nitrogen in the residue insoluble in water, as directed in $§ 96$. If the latter is lower, it is to be regarded as the more accurate of the two; the

1 Not more, otherwise starch is attacked. 
explanation of this is to be found in the fact alluded to in $\$ 92$ et seq., viz., that the material treated according to $\S 103$ has been exhausted with ether and alcohol previous to being extracted with water, and that therefore the quantity of albuminoids taken into solution is smaller than that extracted according to $\S 92$. But since the soluble albuminoids are determined in material that has not been subjected to the action of ether, etc., it follows that the nitrogen in the residue after exhaustion with water should guide us in estimating the insoluble albuminoids.

It should be observed that one extraction with dilute caustic soda is often insufficient to remove all the substances soluble in that menstruum. The treatment should therefore be repeated a second and, if necessary, third time.

§106. Albuminoids not Dissolved by Dilute Soda.-There still remains the question whether the assumption is admissible that all albuminoids insoluble in water are dissolved by the dilute caustic soda used in $\S 103$. I can only reply that in a large number of experiments made by Stackmann, Koroll, and CramerDolmatoff, ${ }^{1}$ the residue after extraction with water, alcohol, and soda was always tested for nitrogen, with the result that in none but substances very rich in suberin could it be said that a little was often present. Of course, it would be possible to apply Lassaigne's test to the residue after extraction with dilute soda; if evidence of nitrogen be obtained, the amount should be estimated and calculated as "albuminoids insoluble in dilute soda." In experiments made by Treffner ${ }^{2}$ on mosses in my laboratory it was found that the amount might occasionally be very considerable. At all events, if nitrogen is present, the quantity should be determined. (Cf. $\$ \S 232,238$.)

$\S 107$. Substances Dissolved by Dilute Soda, not Precipitated by Alcohol.-The filtrate and washings from the precipitate obtained in $\$ 103$ are evaporated to dryness, and the calculated amount of acetate of soda deducted from the dried residue. (See $\$ 237$.) The remainder represents the substances soluble in dilute caustic soda, not precipitated by acetic acid and alcohol. If the residue dissolves completely in a few cc. of water it may be concluded that no substance allied to phlobaphene, soluble in alcohol, is present. In that case the organic matter (apart from the acetate

${ }^{1}$ See the dissertations, etc., subsequently quoted.

2 Dissertation. Dorpat, 1881. 
of soda) is sometimes a decomposition product of metarabic acid or of allied mucilaginous substances. The action of caustic soda on the latter often results in the formation of products that are not precipitable by alcohol. But this body that thus remains in solution on adding alcohol will be more often found to belong to the albúminoids. (See $\S 235$.)

$\S 108$. Phlobaphene.-A brown residue insoluble in water would frequently consist of phlobaphene. (See also § 48.) It should be collected on a tared filter, washed, dried, weighed, and deducted from the evaporation-residue in $\S 106$ before the weight of the substances derived from mucilage, caseine, etc., can be arrived at. (See also § 246.)

The polyporic acid, isolated by Stahlschmidt, ${ }^{1}$ may also be mentioned here. It is insoluble in water, ether, benzene, bisulphide of carbon, and glacial acetic acid, sparingly soluble in warm chloroform, alcohol, and amylic alcohol, but dissolved by dilute ammonia, forming a violet liquid, from which it is precipitated by hydrochloric acid. It crystallizes in rhombic plates, and melts at about $300^{\circ}$.

'Humus.'-I am convinced that the 'humus' mentioned in old plant-analyses was in reality partly phlobaphene and its decomposition-products. In the majority of vegetable substances humus is not to be found, unless they are already in a state of decomposition. Perhaps some thick barks and lignified fungi might yield substances with characters resembling those possessed by humus. To solvents such substances would, it is true, show a behaviour similar to that of the phlobaphenes; but in distinguishing them we may take advantage of the fact that the majority of the so-called humic substances contain hydrogen and oxygen in the proportion in which they exist in water, and that humus does not yield the decomposition-products mentioned in $\S 42$ when acted upon by fused caustic potash.

1 Annal. d. Chem. und Pharm. clxxxvii. 177 (1877) (Journ. Chem. Soc. xxxii. 620 ). 
VII.

Examination of Substances Soluble in Dilute Hydrochloric Acid ; Pararabin, Oxalate of Calcium, etc., and STARCH.

§109. Method of Extraction. - The insoluble residue from $\$ 103$ is washed with water (which is best accomplished either by decantation or as directed in $\S 71$ ), and suspended in water containing 1 per cent. of hydrochloric acid. It is advisable to adhere to the same proportion of menstruum to material as already recommended. The method of procedure now depends mainly upon the presence or absence of starch and of pararabin (or allied substance). The former may be recognised under the microscope by the blue colour the granules assume when treated with an aqueous solution of iodine. ${ }^{1}$

$\$ 110$. Estimation of Oxalate of Calcium.-The simplest case would be that in which neither starch nor pararabin is present. The only object in digesting with dilute hydrochloric acid would then be to extract oxalate of calcium. To effect this the digestion should be continued for about twenty-four hours at a temperature of $30^{\circ}$. A measured quantity of the filtrate may be neutralized with ammonia, or mixed with a known quantity of acetate of soda sufficient to convert all the hydrochloric acid into chloride of sodium. The oxalate of calcium, which separates out insoluble

${ }^{1}$ If large quantities of mucilaginous (? albuminous) substances are present, this colouration is not perceptible on directly moistening a transverse section. with iodine water. The mucilage (or albumen) must be first removed by treatment with a dilute $(0.1$ per cent) solution of caustic soda. A stronger solution should not be employed, as it might act upon the starch itself. If the residue from $\S 103$ is examined, the treatment with alkali is of course unnecessary. For a classification of starches, according to the shape of the granule, see Nägeli's 'Monographie der Stärkekörner,' Basel, 1858 ; and Vogl, Zeitschr. d. österr. Apoth. Ver. 1866, pp. 290, 310. 
in acetic acid, is allowed to settle, and when the supernatant liquid is perfectly clear it is poured off, and the precipitate transferred to a fine filter, washed and dried. It may then be converted either into carbonate by gentle, or into oxide by strong ignition, and from the weight of either the amount of oxalate calculated. The filtrate and washings are evaporated to dryness, and the residue weighed. As the amount of chloride of sodium and undecomposed acetate is known, it will thus be ascertained if other substances (albuminoids, $\$ 223$ et seq.) have been dissolved by dilute hydrochloric acid.

Instead of estimating the oxalate as carbonate or oxide, the washed precipitate may be dissolved in dilute sulphuric acid, and the oxalic acid determined by titration with permanganate of potassium. (Cf. $\S 81,219$.)

Microscopical Examination.-Oxalate of calcium is always deposited in plants in the crystalline condition, and its presence may therefore be confirmed by microscopic examination. The crystals must be insoluble in water, alcohol, and ether, but soluble in dilute hydrochloric acid.

It should also be ascertained, by means of the microscope, if all the oxalate has been dissolved by the treatment directed in $§ 109$. If that is not the case, the maceration with dilute acid should be repeated.

$\S 111$. Estimation of Oxalate of Calcium and Pararabin.-If the oxalate of calcium is accompanied by pararabin, but not by starch, the maceration is continued for twenty-four hours as before; but previous to filtering, the whole is rapidly raised to the boilingpoint in a flask provided with an upright condenser. A measured quantity of the filtrate (filtered whilst hot) is neutralized with ammonia, and mixed with 2 to 3 volumes of 90 per cent. alcohol. The precipitate, which contains oxalate of calcium and pararabin, is collected on a tared filter, washed with 60 to 70 per cent. alcohol, dried, and weighed. It is then incinerated, the ash calculated to oxalate of calcium, and deducted from the weight of the precipitate. The remainder is the weight of the pararabin.

The filtrate and washings from the precipitate may be evaporated to dryness as directed in $\$ 110$, in order to ascertain if other substances have been dissolved. Here, too, albuminous substances may possibly be found, and they may also be present in the precipitated pararabin. Should that be the case, they may be 
estimated by determining the nitrogen in a portion of the precipitate. (Cf. § 233.)

$\S 112$. Estimation of Pararabin alone.-If pararabin ${ }^{1}$ alone is present, the estimation may be conducted as described in $\S 111$, with the exception, of course, that the determination of calcium is unnecessary. After precipitation with alcohol, pararabin swells in contact with water, but does not dissolve unless an acid be added. It is precipitated by alkalies, and does not yield arabinose under the influence of dilute sulphuric acid. (Cf. § 245.)

\$113. Estimation of Starch and Oxalate of Calcium.-If pararabin is absent, but oxalate of calcium and starch are present together, the material under examination may be boiled (not digested on a water-bath) with 1 per cent. hydrochloric acid for four hours in a flask provided with an upright condenser. The flask is weighed before and after boiling, and any water that may have been lost by evaporation replaced. In one portion of the filtered liquid the oxalate of calcium may be determined as directed in $\S 110$, and in another the glucose produced from the starch estimated by titration with Fehling's solution ( $\$ 83$ ).

The modification necessary when starch alone is present needs no special description.

\$114. Estimation of Oxalate of C'alcium, Starch, and Pararabin.The following is the method I have adopted when oxalate of calcium, starch, and pararabin are present together. Water is added to the substance under examination in the proportion of $10 \mathrm{cc}$. for every gram, and the whole brought to the boiling-point. After cooling to $40^{\circ}$ or $50^{\circ}$, a centigram or more of good, active diastase is added, and the maceration continued at the same temperature until the starch-paste is completely liquefied. The residue, after filtering and washing, is treated according to $\S 111$. A measured quantity of the filtrate containing the maltose and dextrin produced from the starch is acidified with 'hydrochloric acid and boiled as directed in $\S 113$, the glucose being finally estimated with Fehling's solution and calculated into starch.

$\S 115$. Estimation of Starch alone.-If a vegetable substance, especially one rich in mucilage, metarabic acid, pararabin, glucosides, etc., is to be examined for starch without previous treat-

${ }^{1}$ Compare Reichardt, Ber. d. d. Chem. Ges. viii. 807 (1875) (Journ. Chem. Soc. xxviii. 1179). 
ment with various solvents, a method that I published in $1861^{1}$ may be adopted by which the substances that accompany the starch are removed. The powdered material is mixed with 30 parts of a 4 per cent. solution of caustic potash in alcohol, and heated to $100^{\circ}$ for a day or two in a well-closed flask. After filtering and washing with spirit till free from alkali, the substance on the filter is exhausted with water; and to effect this it is advisable to transfer it to a beaker. The residue insoluble in cold water is boiled with dilute hydrochloric acid, and treated as directed in $\S 113$. The caustic potash acts upon the foreign substances which interfere with the direct estimation of the starch, rendering them soluble partly in alcohol, partly in water, whilst the starch itself is not attacked. (See $\S 243$.)

1 Journ. f. Landwirthsch (May, 1862), and Pharm. Zeitschr. f. Russland, i. 41. For the estimation of starch as glucose after the action of dilute sulphuric acid, see Musculus, Chem. Centralbl. 1860, p. 602 (Am. Journ. Pharm. xxxii. 433) ; and Philipp, Zeitschr. f. anal. Chem. N. F. iii. 400. Sachsse (Zeitschr. f. anal. Chem. xvii.231, 1878; Year-book Pharm. 1878, 97), has shown that the inversion is better effected by hydrochloric acid-1 per cent. of the weight of the liquid. Both Sachsse and Nägeli found that the analyses of starch were more accurately expressed by the formula $6 \mathrm{C}_{6} \mathrm{H}_{10} \mathrm{O}_{5}+\mathrm{H}_{2} \mathrm{O}$, than by that usually adopted, viz., $\mathrm{C}_{6} \mathrm{H}_{10} \mathrm{O}_{5}$. 
VIU.

Deterifination of Lignin and Aluied Suptastances and of Cellulose.

\$11ô. Lignin, Incrusting and Cuticular Substances. -The residue of the powder insoluble in all the foregoing menstrua, after treatment as directed in $\S 109$, is washed with water, dried, and weighed. After having been again finely powdered, it is macerated in freshly prepared chlorine-water (in the proportion of about $100 \mathrm{cc}$. for every gram of substance), until the colour changes to a pale yellow. If 2 to 3 days do not anffice, the chlorinewater must be drawn off and replaced by fresh and this treatment repeated if necessary. It is finaliy colle sed on a tared filter, and washed first with water, then with ver dilute $(0.3$ per cent.) solution of caustic potash until the washin s are colourless, the alkali being ultimately removed by pure wer. The loss in weight after drying represents the amount of $l i$ hin, the so-called incrusting substances, the majority of the suberin and cuticular substance. (Cf. § 247.) Bromine-water has been proposed in the place of chlorine-water, but it does not act so energetically.

With regard to the microchemical examination, I may, observe that lignified tissues absorb fuchsin from its aqueous solution, and retain it so tenaciously that they appear stained deep-red even after maceration in glycerine, which removes all the colouring matter from non-lignified tissue. Russow ${ }^{1}$ recommends the object to be placed on a slide w th a drop of dilute aqueous solution of aniline-red. A drop of gtycerine is then brought into contact with the edge of the coverslip on the slide, and left for twentyfour hours. Stiles ${ }^{2}$ macerates in a dilute solution of chlorinated lime ( 1 in 60 ), then transfers for an hour to a solution of hypo-

1 Sitz-ber. d. Dorpater Naturf. Gesellsch. 1880, p. 419.

2 Pharm. Journ. and Trans. [3], vi. 741. 
sulphite of soda (1 in 32), washes with alcohol, and finally removes to a dilute alcoholic solution of acetate of rosaniline ( 1 in 960), the excess of which is washed out with spirit. Aniline-blue is said to impart a fine blue or violet colour to the parenchyma of the medullary rays, etc. The solution is made by dissolving 0.0325 gram of aniline-blue in 3.88 gram of water, adding 0.5 gram of strong nitric acid and spirit to 48 grams. After staining red as directed by Stiles, the section may be immersed for a few minutes in the solution of aniline-blue, washed with spirit and finally treated with cajeput oil or turpentine.

Wiesner ${ }^{1}$ has described a qualitative reaction for woody tissue, which consists in moistening the section with a 0.5 per cent. solution of phloroglucin, and subsequently treating with hydrochloric acid. The lignified tissue assumes a reddish or violet colour.

§.117. Estimation of Cellulose.-The residue, after treating as directed in $\S 116$ and weighing, is a mixture of cellulose, intercellular substance, remains of the cuticular substance, etc., together with a little ash (and possibly also sand). It may be removed from the filter (which should be reserved), powdered, and introduced into a flask containing 50 to $100 \mathrm{cc}$. of nitric acid (sp. gr. $1 \cdot 16$ to $1 \cdot 18$ ) ; 1 to 2 grams of chlorate of potash are then added, and the mixture allowed to stand in a cool place with occasional agitation until the insoluble matter appears almost white. If this is not effected in a day or two the mixture may be warmed for one or two hours to about $40^{\circ} \mathrm{C}$. (not higher), and again allowed to stand. If this is not successful the strength of the nitric acid may be increased until it reaches a specific gravity not exceeding 1:20. After the action of the acid has been continued long enough, it may be diluted with water and filtered, taking care to pour the supernatant liquid on to the filter, leaving the insoluble matter as long as possible in the flask. After washing free from acid, it is treated with dilute ammonia ( 1 in 50 of water) as long as that is coloured brownish, and finally with alcohol and, if necessary, with ether. The residue is dried and weighed. The loss in weight usually represents intercellular substance and certain carbohydrates allied to cellulose, but less resistent (hydrocelluloses), etc. (See $\S \S 245,246$.) The residue on the filter consists of cellulose with a little ash (silica, sand, etc.), that may be estimated and deducted. (See also § 248.)

1 Zeitschr. f. anal. Chem. xvii. 511, 1878 (Journ. Chem. Soc. xxxiv. 612). 


\section{CONCLUDING REMARKS.}

$\S 118$. In compiling the foregoing method of analysis, one object that I had in view was to show how, when working upon a small quantity of material, say 30 to 50 grams, an insight into its composition might be gained, so that at least the presence or absence of the more important constituents of plants might be ascertained. I wished to show further how the constituents actually present might be estimated, even if no more than the above-mentioned quantity was available. I had therefore to devise a combination of qualitative and quantitative analysis, and the fact that a considerable number of the same constituents occur in the majority of plants justified me in making the attempt.

Means have also been indicated by which attention would be drawn to the presence of substances that occur only in single plants or in smaller groups of the vegetable kingdom. In this respect the foregoing method is of course but an introduction, the special application and perfection of which for each separate case must be left to the investigator himself. Processes for the quantitative estimation of certain substances, and especially such as are of considerable practical importance in medicine, agriculture, etc., have already been recommended, and will be followed by others in the second part of the work.

$\S 119$. It must be admitted that many of the proposed methods of detection and estimation cannot boast of the accuracy attainable in the analysis of some inorganic substances. For this reason I advise beginners to refrain from calculating their analyses, as is frequently done, to the fourth and even fifth place of decimals. Such calculations often mislead readers less acquainted with the subject to attach to the separate determinations an importance to which they are not entitled. I consider it ample to carry the calculations to the second decimal place.

To those who ask of what use analyses are, the accuracy of which I have myself this moment questioned, I reply that the object of analyzing a vegetable substance, as for instance ergot, is not so much to ascertain the exact composition of a fungus produced on a certain ear of rye in a certain field, but to obtain information as to the approximate composition of ergot in general, the specimen under examination being taken as a representative of the drug. Attention must be specially drawn to the fact that 
in different years and different localities the proportions in which the constituents of ergot occur present certain variations.

If, on the other hand, an approximate analysis is not required, but in its stead a fairly accurate comparison of specimens gathered in different fields, then it must be borne in mind that only certain practically valuable constituents have to be taken into account, for the estimation of which more accurate methods may not unfrequently be devised. This we are generally able to accomplish, for we are in a position to elaborate the necessary mode of treatment, to determine the extent of the errors involved, and the corrections to be made for them, and to make several estimations from the same material from which a mean may be calculated. 
IX.

Special Methods for the Estmation of certain Constituents of Plants, Supplementary Notes to the PreCEDING EXPERIMENTS.

FATS AND THEIR CONSTITUENIS; CHOLESTERIN, FILICIN, ETC.

$\S 120$. Estimation of Fixed Oils.-For reasons given in $\S 8$, I recommended the use of benzene some twenty years agol for extracting fixed oils. Petroleum spirit, which I sulsequently introduced for the same purpose, has the advantage over benzene of being more volatile and possessing a lesser solvent power for resins, etc. (Cf. § 36.) The use of benzene was afterwards advocated by Hoffmann ${ }^{2}$ also, who gave it the preference over ether and bisulphide of carbon. Other methods for estimating fixed oils have been described by Münch. ${ }^{3}$ Various forms of apparatus that may be used have formed the subjects of communications from Storch, ${ }^{4}$ Wagner, ${ }^{5}$ Simon, ${ }^{6}$ Tollens, ${ }^{7}$ Schulze, ${ }^{8}$ Tschaplowitz, ${ }^{9}$ Medicus, ${ }^{10}$ Siewert, ${ }^{11}$ Hirschsohn, ${ }^{12}$ Keyser, ${ }^{13}$ and others.

The apparatus represented in Fig. 5 is that last devised by Tollens. It consists of a weighed flask, A, holding about $100 \mathrm{cc}$., to which is tightly fitted, by means of a perforated cork, a glass tube $\mathrm{B}$; the latter is about $30 \mathrm{~mm}$. in diameter at its upper, and

${ }^{1}$ Pharm. Zeitschr. f. Russland, i. 44, 1862 ; Anm. Zeitschr. f. anal. Chem. i. 490 .

2 Zeitschr. f. anal. Chemie, vi. 368, $186 \%$.

${ }^{3}$ N. Jahrb. f. Pharm. xxv. 8, 1866.

${ }^{4}$ Zeitschr. f. anal. Chemie, vii. 68, $1868 . \quad{ }^{5}$ Ibid. ix. 354, 1870.

${ }^{6}$ Ibid. xii. 179, 1873 (Journ. Chem. Soc. xxvii. 293).

7 Ibid. xiv. 82, 1875, and xvii. $320,1878 . \quad{ }^{8}$ Ibid. xvii. 174, 1878.

${ }^{9}$ Ibid. xviii. 441, 1879.

${ }^{10}$ Ibid. xix. 163, 1880.

11 Landw. Versuchsst. xxiii. 317, 1879 (Journ. Chem. Soc. xxxvi. 558).

12 Archiv d. Pharm. [3], x. 486, 1877. 13 Farm. Tidskr. 1,880, No\$, 9 and 19. 


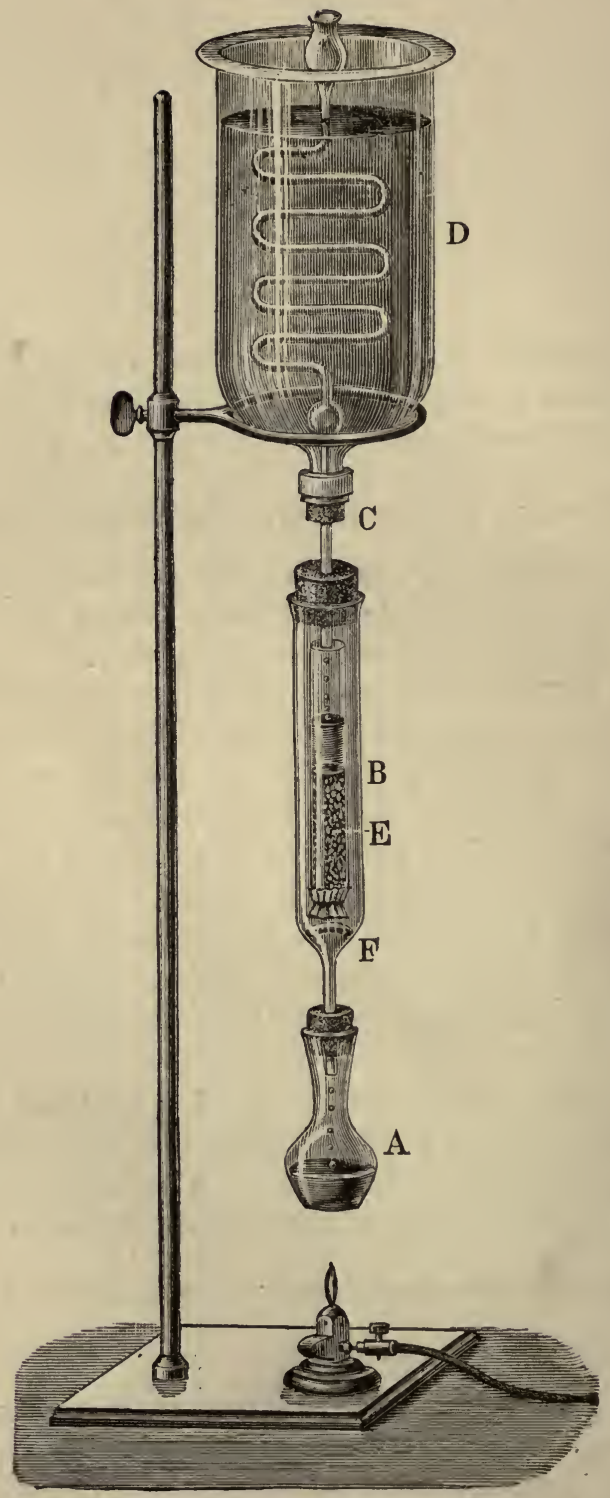

Fig. 5. 
5 to 7 at its lower extremity; the former communicates by means of perforated corks with the condenser D. A glass tube, E, about $20 \mathrm{~mm}$. in diameter, is supported upon a bent glass rod, F, in such a manner that the condensed vapour from $\mathrm{C}$ drops directly into it. In this tube, $\mathrm{E}$, the substance to be examined is carefully packed; a piece of filtering-paper is then tied over the lower end, and a small circular filter laid upon the surface of the packed substance. During the extraction the heat is so regulated that the material is constantly covered by a layer of ether 1 to $2 \mathrm{~cm}$. thick.

$\S 121$. Resinification.-The rapidity with which an oil resinifies may be ascertained by exposing it to the air in thin layers and noting the daily increase in weight. Parallel experiments should be made with almond and linseed oil under precisely similar conditions. The oil used should be quite free from any trace of petroleum spirit.

$\S 122$. Elaidin Test.-This test $(\$ 12)$ consists in passing nitrous acid into a few cc. of the oil and observing the length of time that elapses before solidification takes place. Another method is to introduce copper turnings or a little mercury, together with nitric acid, into a test-tube, and pour a few cc. of the oil upon the mixture. The colour also of the elaidin produced may be characteristic of the oil under examination.

Using 5 grams of nitric acid, sp. gr. 1*4, and 1 gram of mercury to 10 grams of oil, Massie ${ }^{1}$ observed the following reactions :

On agitating the oil with the nitric acid alone for two minutes and allowing the liquids to separate, the following colourations were observed : almond, hazelnut, sunflower-seed oil, colourless or slightly greenish; olive oil, greenish, white or slightly yellowish-green, or distinctly green; ground-nut oil and poppy-seed oil, reddish; castor and sesamé oil, yellowish or yellowish-orange; oil of white mustard, apricot, walnut, camelina, beech, rape and linseed oil, cherry-red or reddish-orange; oil of black mustard, cotton and hemp-seed oil, brown or brounish-red.

The acid was coloured yellowish by olive oil (occasionally), saffron-yellow by sesamé, light brown with cotton, and slightly reddish or greenish by hemp oil.

After the addition and solution of the mercury, the mixture is

1 Journal de Pharm. et de Chim. [4], xii. 13, 1869. 
shaken at intervals and finally set aside. The following observations were made :

After 20 to 30 mins.

Almond

Hazelnut

Sunflower-seed

Olive

Ground-nut

Poppy

Castor

Sesamué

Apricot

White mustard

Camelina sativa

Walnut

Beech

Rape

Colza

Linseed

Black mustard

Cotton-seed

Hemp white or pale-greenish

lemon-yellow
pale yellowish
pale reddish
red
rose
yellowish-orange
red
yellowish-orange
cherry-red
orange
reddish-yellow
pale reddish
reddish-brown (effervesces)
pale reddish
dark orange-red or reddish
brown

white.

After $1 \mathrm{hr}$.

"

lemon-yellow.

pale yellowish-green.

pale reddish.

red.

yellow.

yellowish-orange.

rose.

reddish-yellow.

reddish-orange.

reddish-yellow.

reddish-orange.

orange-yellow.

pale yellowish-orange.

reddish-brown.

reddish-yellow.

pale orange-red or red.

reddish-brown.

The following oils solidify : almond in $1 \frac{1}{4} \mathrm{hr}$, hazelnut in $1 \mathrm{hr}$., olive oil in $1 \mathrm{hr}$., ground-nut in $1 \frac{3}{4} \mathrm{hr}$., sesamé in $2 \frac{1}{2} \mathrm{hr}$., apricot in $1 \frac{3}{4} \mathrm{hr}$., beech in $6 \mathrm{hr}$., rape in $3 \mathrm{hr}$., colza in $3 \frac{1}{2} \mathrm{hir}$., cotton in $1 \frac{3}{4} \mathrm{hr}$. ; the remainder do not solidify at all.

\$123. Behaviour to Sulphuric Acid.--Casselman ${ }^{1}$ observed the following rise in temperature when 50 cc. of the oil were mixed with 10 cc. of conc. sulphuric acid :

Linseed from $14^{\circ}$ to $132^{\circ}$ to $134^{\circ}$.

$\begin{array}{llll}\text { Sunflower } & , & , & 92^{\circ} \text {. } \\ \text { Poppy } & " & " & 92^{\circ} \text {. } \\ \text { Olive } & " & " & 48^{\circ} \text {. } \\ \text { Almond } & " & " & 59^{\circ} \text {. }\end{array}$

With the oil from peony-seed Stahre and myself ${ }^{2}$ observed a rise to $68^{\circ}$, whilst almond oil rose to $48^{\circ}$.

\$124. Behaviour to Reagents mentioned in $\$ 12 .-$ Casselman has made the following observations with the reagents mentioned in $\S 12$ :

1 Pharm. Zeitschr. f. Russland, 299, 1867 ; Zeitschr. f. anal. Chemie, vi. 479. See also Chateau.

${ }^{2}$ Archiv d. Pharm. [3], xiv. 412, 531, 1879 (Journ. Chem. Soc. xxxvi. . 1043). 
§124. BEHAVIOUR TO REAGENTS.

\begin{tabular}{|c|c|c|c|c|c|c|c|}
\hline 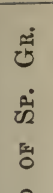 & $\underset{\dot{\sim}}{\stackrel{\leftrightarrow}{\dot{n}}}$ & 言 & 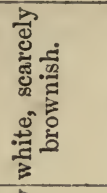 & 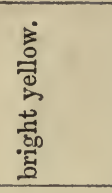 & 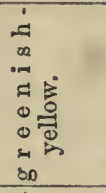 & 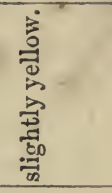 & 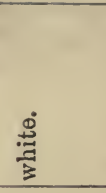 \\
\hline 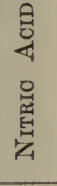 & 㐫 & 言 & 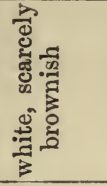 & 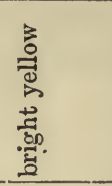 & $\begin{array}{l}\dot{a} \\
\infty \\
\ddot{a} \\
0 \\
0 \\
0 \\
\infty \\
\infty \\
\infty\end{array}$ & 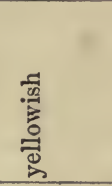 & $\stackrel{0}{\stackrel{\Xi}{*}}$ \\
\hline \multirow{2}{*}{ 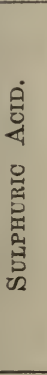 } & 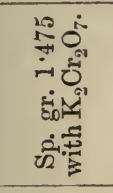 & 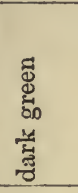 & 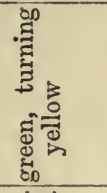 & हี & 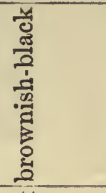 & 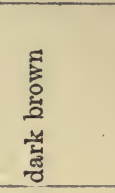 & 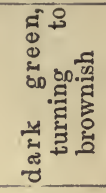 \\
\hline & 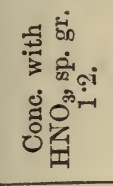 & 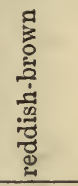 & 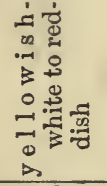 & ह్ & 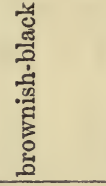 & 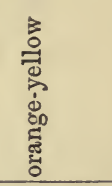 & 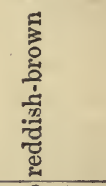 \\
\hline \multirow{3}{*}{ 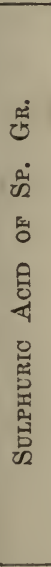 } & 咅 & 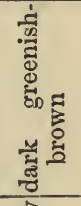 & 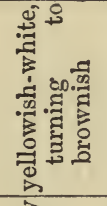 & $\begin{array}{l}0 \\
0 \\
0 \\
0 \\
0 \\
0.03 \\
0 \\
0 \\
0 \\
0 \\
0 \\
0\end{array}$ & 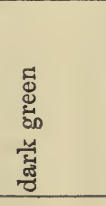 & 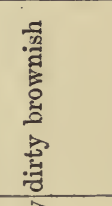 & 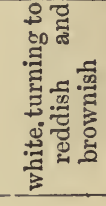 \\
\hline & 宊 & 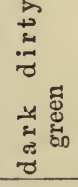 & 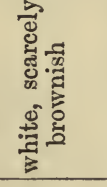 & 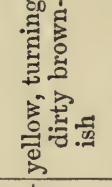 & 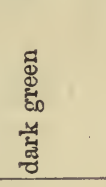 & 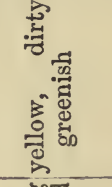 & $\stackrel{8}{:}$ \\
\hline & $\frac{10}{\overbrace{}^{\circ}}$ & 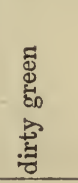 & 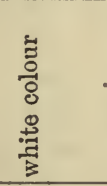 & 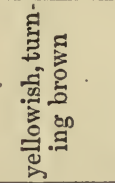 & 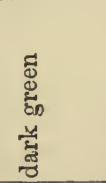 & 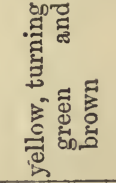 & 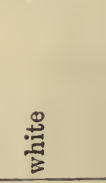 \\
\hline & \multirow[t]{2}{*}{ 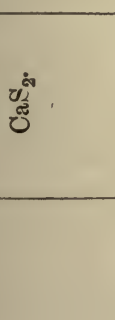 } & 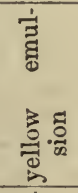 & 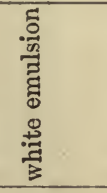 & 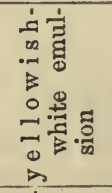 & 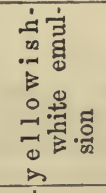 & 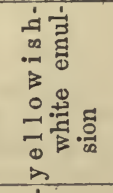 & 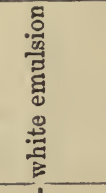 \\
\hline & & 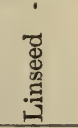 & 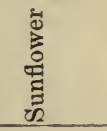 & 产 & 駕 & 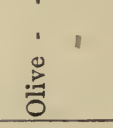 & 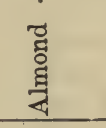 \\
\hline
\end{tabular}


Stannic chloride produces the following changes in colour: linseed, dirty yellow, passing to green; sunflower, white, turning brown; poppy, greenish ; hemp, yellowish-green ; olive, bright yellow; almond, scarcely yellowish. On warming with chloride of zinc linseed oil becomes green, and hemp oil assumes a fine green colour, while the remainder undergo no change. Syrupy phosphoric acid forms a sort of emulsion with linseed and poppy oil, but not with the others.

Warming with mercuric nitrate colours linseed oil from dark green to brownish-red ; sunflower, bright yellow ; poppy and hemp oil, green, turning brown; olive, dark yellow, passing to orange red; almond, deep chrome.

Bieber ${ }^{1}$ used nitric acid of sp. gr. 1·4, and also a cooled mixture of equal parts of conc. sulphuric and fuming nitric acid in the proportion of 1 volume of reagent to 5 of oil.

Hauchecorne ${ }^{2}$ has published reactions of oils with peroxide of hydrogen, but without specifying the strength of the reagent. He states that on shaking 1 volume of solution of peroxide of hydrogen with 4 of oil, olive assumes an apple-green; poppy, a flesh colour ; sesamé, bright red ; ground-nut, greyish-yellow ; and beech-nut oil, an ochre red. According to Cohné, drying oils may be distinguished from non-drying by their behaviour to peroxide of hydrogen. The former are said to be quickly decomposed with separation of fatty acids, whilst the latter resist such treatment.

Basoletto ${ }^{3}$ observed that sesamé oil, when shaken with an equal volume of hydrochloric acid (23 to 24 per cent) containing 2 per cent. of cane-sugar, assumed a reddish tinge, passing to cherry-red, whilst olive oil was not coloured. On agitating with nitric acid containing sugar, sesamé oil was coloured cinnamon, whilst the acid became yellowish-green. Cotton-seed oil turns yellow with the same reagent (the acid becoming pale rose coloured), but

${ }^{1}$ Apotheker Zeitung, xii. 161, 1877 (Journ. Chem. Soc. xxxiv. 343). For the action of nitric acid on fatty oils, see also Hauchecorne, Zeitschr. f. anal. Chemie, iii. 512, 1864, where, however, the strength of the acids employed is not mentioned. Langlies (ibid. ix. 534, 1870) recommends mixing nitric acid specific gravity 1.4 with $\frac{1}{3}$ of its volume of water, and warming 1 part of this reagent with 3 of oil in the water-bath. Sesamé oil is said to yield a red mass by this treatment.

2 Zeitschr. f. anal. Chemie, ii. 442, 1863.

${ }^{3}$ Bulletin della Soc. Adriatic, i. 178, 1875. 
almond and castor oil produce no alteration. According to Vidan ${ }^{1}$ hydrochloric acid containing sugar changes the colour of castor oil to orange-yellow, poppy oil yellowish-brown, groundnut oil intense yellow, olive oil yellowish-orange, rape oil dark brown, and almond oil yellowish-orange.

For the use of chloride of antimony as a reagent see Zabludowski ${ }^{2}$ and $\mathrm{Walz}^{3}$ The latter found that on adding a few drops of the reagent, which should be of a syrupy consistence, to 2 or $3 \mathrm{cc}$. of the oil to be examined, olive oil formed a whitish emulsion, gradually turning dark, without any rise in temperature, whilst with cotton-seed oil a considerable amount of heat was evolved, the mixture becoming solid and of a chocolate-brown colour.

Concentrated solution of chlorinated lime is said to form an emulsion with 8 times its volume of poppy oil, but not with almond oil.

Caustic soda of specific gravity 1·33, heated to boiling with 4 or 5 times its volume of oil, yields a white liquid mixture with castor oil, yellowish-white with sesamé, colza, poppy, and walnut, and yellow with linseed, whilst olive oil and hemp yield respectively brownish and brownish-yellow solid masses. ${ }^{4}$ Some oils, such as rape and colza, may be contaminated with sulphur compounds, which may be detected by nitro-prusside of sodium after treatment with caustic soda.

For the use of the spectroscope in identifying fixed oils see Gilmour, ${ }^{5}$ of the polariscope see Buignet, ${ }^{6}$ of cohesion figures see Tomlinson, ${ }^{7}$ Kate Crane, ${ }^{8}$ and Moffat. ${ }^{9}$

$\$ 125$. Free Fat-acid.-The presence of free fat-acid may be

1 Journ. de Pharm. et de Chim. xxii. 30, 1875 (Journ. Chem. Soc. xxix. 111). Compare also Jahresb. f. Pharm. 288, 1875. The hydrochloric acid and sugar reaction was recommended by Camoin as early as 1860. Compare Choulette, 'Observations prat. de Chim. et de Pharm.' Fasc. i. 130.

${ }^{2}$ Pharm. Zeitschr. f. Fiussland, ii. 233, 1863.

${ }^{3}$ Amer. Journ. Pharm. xlvi. 25, 1874.

' Compare Hager, 'Untersuchungen' (Günther Leipsic, 1874), vol. ii. 510.

${ }^{5}$ Pharm. Journ. and Trans. [3], vi. 981, and Jahresb. f. Pharm. 362, 1876.

${ }^{6}$ Journ. de Pharm. et de Chim. xl. 252, 1862 (Amer. Journ. Pharm. xxxiv. 140).

7 Pharm. Journ. and Trans. [2], v. 387, 495.

${ }^{8}$ Ibid. [3], v. 243, and Jahresb. f. Pharm. 289, 1874.

${ }^{9}$ Chem. News, xviii. 473 . 
detected, according to Jacobson, ${ }^{1}$ by shaking with powdered rosaniline. Oil containing free fat-acid is coloured red.

Rumpler ${ }^{2}$ employs carbonate of soda, which does not emulsify oils containing no free fat-acid.

Geissler $^{3}$ estimates the free fat-acid by diluting with 2 or 3 volumes of ether and titrating with alcoholic potash, using an alcoholic solution of rosolic acid or phenol-phthalein as an indicator.

§ 126. Cholesterin. Detection and Estimation.-Hoppe-Seyler" ${ }^{4}$ -detects and estimates cholesterin in vegetable substances by extracting with ether, distilling, boiling the residue for a few hours with alcoholic potash, evaporating, redissolving in water, and shaking with ether. If the cholesterin obtained by evaporating the ethereal solution is not pure, the treatment with alcoholic potash is repeated. If sufficient alkali is present neither fat nor soap will be taken up by the ether.

Schulze ${ }^{5}$ directs attention to the fact that the estimation is inaccurate if the material contains vegetable wax yielding an alcohol (§14) on decomposition with an alkali, on account of the influence the latter exercises on the solubility of cholesterin in spirit. Schulze recommends the conversion of the impure cholesterin into benzoate of cholesteryl by heating with benzoic acid in sealed tubes. This compound may be freed from many foreign substances by boiling with absolute alcchol, in which it is almost insoluble. After recrystallization from ether the cholesterin may be liberated by heating with alcoholic potash.

Cholesterin is soluble in petroleum spirit as well as in ether, and is therefore extracted by the former, together with the fixed oil. If an accurate estimation is required, large quantities of material must be worked upon, as cholesterin occurs in only small proportions in vegetable substances. (Beneke obtained 1.5 gram from 2,500 grams of grey peas.) It is insoluble in water, crystallizes from alcohol in silky needles and plates (belonging to the rhombic system), melts at $137^{\circ}$, and is, in alcoholic solution, lævo-rotatory $\left(\alpha_{\mathrm{D}}=36 \cdot 61^{\circ}\right)$. Warmed with a mixture of 1 vol.

${ }^{1}$ Chem.- tech. Repert. i. 84 ; Zeitschr. f. anal. Chemie, xvii. 387, 1878.

2 Zeitschr. f. anal. Chemie, ix. 417, 1870.

3 Ibid. xrii. 387,1878 (Journ. Chem. Soc. xxxiv. 334).

${ }^{4}$ Med.-chem. Unters. Heft i. 143. Zeitschr. f. anal. Chemie, v. 422, 1866.

${ }_{5}^{5}$ Zeitschr. f. anal. Chemie, xvii. 173, 1878. (Journ. Chem. Soc. xxxiv.612.) 
conc. sulphuric acid with 1 of water, a red colouration is produced, whilst 4 of acid with 1 of water develops a blue, and 3 with 1 a violet tinge. If a mixture of concentrated hydrochloric acid and solution of ferric chloride (3 in 1) is evaporated with a little cholesterin, a reddish-violet or bluish-violet colour makes its appearance. Similar treatment with sulphuric acid and ferric chloride leaves a carmine residue, which gradually passes to violet and becomes scarlet on treating with ammonia. ${ }^{1}$ After trituration with sulphuric acid cholesterin is coloured red by the addition of chloroform.

Phytosterin, a substance allied to and probably homologous with cholesterin, was discovered by Hesse ${ }^{2}$ in the Calabar bean. Its solubility is, on the whole, similar to that of cholesterin, with which it has occasionally been confounded. It melts at $133^{\circ}$, and is somewhat less powerfully lævo-rotatory $\left(\alpha_{\mathrm{D}}=34 \cdot 2^{\circ}\right)$.

Filicin is another substance soluble in petroleum spirit; it is extracted, therefore, together with the fixed oil, and is partially cleposited in crystals on evaporating such a solution; an appreciable quantity, however, remains dissolved in the fixed oil. Experiments made at my instance by Kruse, ${ }^{3}$ with the object of devising a quantitative separation of filicin from fixed oil, were unsuccessful; all the liquids employed (acetone, acetic ether, ether, heavy petroleum oils, bisulphide of carbon, etc.) dissolved both substances. Attempts to separate the fixed oil from the filicin by dissolving in a hot aqueous solution of carbonate of soda and fractionally precipitating with hydrochloric acid, as well as the same treatment of an alkaline alcoholic solution, were attended with negative results.

The kosin ${ }^{4}$ contained in cousso is soluble in petroleum spirit, especially when warm. It is more easily soluble in ether, benzene, or bisulphide of carbon, somewhat sparingly in alcohol and glacial acetic acid. Ferric chloride colours the alcoholic solution red, and

${ }^{1}$ Zeitschr. f. anal. Chemie, xvii. 17j, 1878, and Ritthausen, 'Eiweisskörper,' 98.

${ }^{2}$ Annal. d. Chem. und Pharm. cxcii. 175, 1878 (Journ. Chem. Soc. xxxiv. 850 ). For paracholesterin, see ibid. ccvii. 229, 1881. Hesse, ibid. ccxi. 283; Schulze and Barbieri, Ber. d. d. Chem. Ges. xv, 953, 1882.

${ }^{3}$ Archiv d. Pharm. [3], ix. 24, 1876 (Journ. Chem. Soc. xxxi. 336). See also Luck, Annal. d. Chem. und Pharm. liv. 191, 1851, and Grabowski, Chem. Centralbl. 409, 1867.

+ Fliickiger and Buri, Archiv d. Pharm. [3], v. 193, 1874 (Pharm. Journ. and Trans. [3], v. 562). 
an alkaline aqueous solution also gradually assumes a red tinge. It is decomposed by fusion with potash, yielding, amongst other substances, butyric acid (the same is the case with filicin).

Euphorbon ${ }^{1}$ is likewise soluble in petroleum spirit, and freely so in ether, benzene, chloroform, acetone, and glacial acetic acid, but not in aqueous alkalies. It dissolves in concentrated sulphuric acid, with the production of a brownish tinge, which is changed to violet by nitre or nitric acid. It melts at $113^{\circ}$ to $114^{\circ}$, and resembles, in many of its properties, lactucon or lactucerin (various species of Lactuca), echicerin (dita bark), and perhaps also cynanchocerin (Cynanchum vincetoxicum and acutum).

Helenin is easily soluble in petroleum spirit, alcohol, and ether, but insoluble in water, even in the presence of a little alkali; it is dissolved, however, by hot concentrated solution of potash. Helenin melts at $110^{\circ}$, crystallizes in colourless needles, and dissolves in conc. sulphuric acid, with production of a red colouration. Hydrochloric acid-gas is also said to colour helenin red. ${ }^{2}$

Coumarin may be recognised by its odour and by its colourless rhombic crystals. It is sparingly soluble in cold, more easily in hot water, and is also dissolved by ether and by alcohol. Amongst the substances it yields when fused with potash is salicylic acid $(\S 26)$. For the allied melilotic acid compare Zwenger. ${ }^{3}$

Styrol also is characterized by its aromatic odour. It is a colourless liquid convertible by long heating in sealed tubes into solid metastyrol. It is almost insoluble in water, but easily soluble in alcohol, ether, and bisulphide of carbon. Heated with chromic acid it yields benzoic acid and other products of decomposition (§26).

For myroxocarpin, see Stenhouse and Scharling ; ${ }^{4}$ for diosmin, Landerer ${ }^{5}$ and Fluckiger $;^{6}$ for kämpferid, Brandes and Jahns $;^{7}$ for asaron (which is soluble at least in warm petroleum spirit),

${ }^{1}$ Hesse, Annal. d. Chem. und Pharm. clxxx. 352 ; clxxxii. 163, 1876 ; cxcii, 193, 1878 (Amer. Journ. Pharm. 1. 552). See also Alberti and Dragendorff, Pharm. Zeitschr. f. Russland, ii. 215, 1863 ; and Flückiger, N. Jahrb. f. Pharm. xxix. 135, 1868.

2 See Kallen, Ber. d. d. chem. Ges. vi. 1506, 1873 (Pharm. Journ. and Trans. [3], vii. 156).

3 Annal. d. Chem. und Pharm. Suppl. v. 100, 1867.

${ }^{4}$ Ibid. Ixxvii. 306, 1851, and xcvii. 69, 1856 (Amer. Journ. Pharm. xxiii. 144).

5 Repert. f. Pharm. lxxxiv. 62.

${ }^{6}$ Ibid. xxiii. (New Series), 102, 1874 (Amer. Journ. Pharm. xlvi. 235).

7 Archiv d. Pharm. lviii. 52; Ber. d. d. chem. Ges. xiv. 2385. 
C. Schmidt ; $;$ for angelicin, which has been proved to be identical with hydrocarotin, see Brimmer $;^{2}$ for carotin, see Husemann. ${ }^{3}$ The last-named substance forms red crystals, soluble in benzene and bisulphide of carbon. It dissolves in conc. sulphuric acid, with a purplish-blue colour, and is also coloured blue by sulphurous-acidgas.

Anemonol, which occurs in many Ranunculacex, may also be mentioned here. It is an oily acrid liquid, volatile with the vapour of water, and gradually changing in aqueous solution to crystalline anemonin. The latter can be isolated by shaking the aqueous solution with ether or chloroform, and like anemonol, acts as an irritant when applied to the skin. ${ }^{4}$

For capsicin and capsaicin see Thresh ; 5 for amyrin and bryoïdin see Buri. ${ }^{6}$

\$127. Caoutchouc.-Petroleum spirit extracts only a trace of caoutchouc, which remains undissolved on treating the residue after evaporation with warm absolute alcohol. If a considerable quantity of caoutchouc is present the majority is left in the substance after exhaustion with petroleum-spirit, and may be extracted by bisulphide of carbon containing 6 to 8 per cent. of alcohol, or by chloroform. From these solutions it may be precipitated by the addition of more alcohol, whilst resinous substances and the like generally remain dissolved. (See also $§ 46$.)

$\S 128$. Estimation of Glycerin ( $\$ 13)$. - For details of the determination of this substance see Reichardt, ${ }^{7}$ and Neubauer and Borgmann. ${ }^{8}$ The latter authors point out the fact that etheralcohol removes other substances besides glycerin from wine, etc., and that the estimation may accordingly be too high. They therefore recommend dissolving the glycerin residue in alcohol, adding 3 volumes of ether, filtering and evaporating. Pasteur advises the evaporation of the solution to be conducted as quickly

${ }^{1}$ Annal. d. Chem. und Pharm. liii. 156, 1845.

${ }^{2}$ N. Repert. f. Pharm. xxiv. 665, 1874 (Pharm. Journ. and Trans. [3], vii. 91).

3 Annal. d. Chem. und Pharm. cxvii. 200, 1861.

${ }^{4}$ Compare Fehling, Annal. d. Chem. und Pharm. xxxviii. 278, 1841; Müller, Chem. Centrlb. 618, 1850 ; Erdmann, Journ. f. prakt. Chem. Ixxv. 209. See Amer. Journ. Pharm. xxxiv. 300 ; xxxi. 440.

${ }^{5}$ Pharm. Journ. and Trans. [3], vi. 941, vii. 473.

6 N. Repert. f. Pharm. 220, 1875 (Pharm. Journ. and Trans. [3], vii. 157).

7 Archiv d. Pharm. [3], x. 408 ; [3], xi, 142, 1877.

8 Zeitschr. f. anal. Chemie, xviii. 442, 1878. See also Pasteur, Annal. d. Chem. und Pharm. Iviii. 330, 1864. 
as possible, as glycerin loses weight even in a vacuum. Compare also Griessmeier and Clausnitzer. ${ }^{1}$

$\S 129$. Wax. - Cetyl alcohol $(\S 14)$ melts at $48^{\circ}$ to $49^{\circ}$, and at $54^{\circ}$ is miscible with spirit of specific gravity 0.812 in all proportions. Cerotyl alcohol melts between $79^{\circ}$ and $81^{\circ}$, melissyl alcohol at $85^{\circ}$. The latter is scarcely soluble in cold alcohol, benzene, petroleum spirit, or chloroform, but dissolves on boiling.

König and Kiesow found a substance in meadow-hay which they considered to be cerotene, or a 'paraffin' of the composition $\mathrm{C}_{20} \mathrm{H}_{42}{ }^{2}$ Hirschsohn has endeavoured to find distinctive characteristics for certain vegetable waxes that find application in the arts, ${ }^{3}$ with the following results :

Wax from Myrica quercifolia.-Soluble in 10 parts of boiling chloroform; the solution remained clear on cooling. Completely soluble in ether. 95 per cent. spirit dissolved 16.16 per cent. at the ordinary temperature; petroleum spirit 53 to 62 per cent. The alcoholic solution gave a precipitate with alcoholic ferric chloride ( 1 in 10 ), which did not dissolve on warming.

Wax from another $s p$. of Myrica yielded 19.88 per cent. to alcohol, $68 \cdot 70$ per cent. to petroleum-spirit. Ferric chloride coloured the alcoholic solution black.

Wax from Myrica cerifera yielded $7 \cdot 16$ per cent. to alcohol and 41.62 per cent. to petroleum spirit. Ferric chloride coloured the alcoholic solution brownish.

Wax from Rhius succedanea (Japan wax) resembled the three foregoing waxes in being completely soluble in chloroform, but was only partially soluble in ether. Aleohol dissolved 14 per cent., petroleum spirit 69.8 per cent. Boiling with 10 parts of 10 per cent. alcoholic potash saponified it ; the soap was completely soluble in 100 parts of water, whilst that from beeswax was only partially dissolved.

Wax from Aleurites laccifera.-The solution in chloroform became turbid on cooling; the addition of an alcoholic solution of acetate of lead to a similar solution of the wax caused a cloudiness on standing. Boiling alcohol left a pulverulent substance undissolved.

1 Ber. d. d. chem. Ges. xi. 292, 1878 (Journ. Chem. Soc. xxxiv. 449), and Zeitschr. f. anal. Chemie, xx. 58, 1881 (Journ. Chem. Soc. xl. 470).

2 Ber. d. d. chem. Ges. vi. 500, 1874. For vegetable wax see also Ludwig, Archiv Pharm. [3], i. 193.

${ }^{3}$ Pharm. Journ. and Trans. [3], x. 749. 
Carnauba wax behaved similarly to chloroform and alcohol, but acetate of lead caused no cloudiness. It was partially soluble in ether; the ethereal solution became turbid on the addition of alcohol. Cold alcohol dissolved 3.25 per cent., petroleum-spirit 5.04 per cent.

Bahia wax resembled carnauba wax in most of its properties, but the addition of alcohol did not render the ethereal solution turbid. Cold alcohol dissolved $9 \cdot 7$ per cent., petroleum spirit 3.32 per cent.

For cerosin from the sugar-cane see Avequin, ${ }^{1}$ Dumas, ${ }^{2}$ and Lewy. ${ }^{3}$

Wax may be recognised microchemically as a solid exudation on the surface of the cells, insoluble in water and partially or wholly soluble in ether. (See also $\$$ 14, 15, 145.)

$\$ 130$. Oleic and Linoleic Acids.-Oudemans ${ }^{4}$ has adopted the following method for the estimation of oleic acid. The soap obtained by saponifying about 10 grams of the fat with potash is decomposed with sulphuric acid; the fat-acids are washed with water, mixed with excess of carbonate of soda and dried. The dry mass is exhausted with boiling alcohol, filtering whilst hot; to the alcoholic solution a little water and an excess of acetate of lead is added. The lead precipitate is collected and dried; and from a weighed portion the oleate of lead is extracted by boiling with ether. The oleic acid may be calculated from the weight of the residue obtained by evaporating the ethereal solution.

Linoleic acil has not yet been isolated in a state of purity, as the free acid when exposed to the air oxidizes even more rapidly than the corresponding glyceryl compound. Mulder estimated it approximately by separating it, together with oleic, palmitic and myristic acid, from the soap, dissolving the mixed fat-acids in alcohol, carefully evaporating, allowing the palmitic and myristic acids to crystallize out, and finally converting into the lead salts. Extraction with ether then removes oleate and linoleate of lead. By repeated evaporation in contact with air and re-solution in ether, the linoleate of lead gradually becomes insoluble, whilst oleate of lead does not change. ${ }^{5}$

1 Annales de Chimie et de Physique, lxxv. 218.

2 Ibid. 238 ; Annal. d. Chem. und Pharm. xxxvii. 170, 1841.

${ }^{3}$ Ibid. (New Series), xiii. 451.

4 Journ. f. prakt. Chem. xcix. 407, 1877.

${ }^{5}$ Compare Zeitschr. f. Chem. ii. 452, 1866 (Amer. Journ. Pharm. xl. 249) ; Schiiler, Jahresb. f. Pharm. 155, 1857. 
Of lauric acid, Oudemans observes that it is easily volatile with the vapour of water, which is not the case with myristic and oleic acid (\$ 15). (Myristic anid other fat-acids may however be distilled in vacuo.)

Oleic and stearic acids may be separated, according to David, ${ }^{1}$ by precipitation from alcoholic solution with glacial acetic acid (1 volume to 3 of 95 per cent. spirit). Oleic acid is not thrown out even by the addition of $2.2 \mathrm{cc}$. of a mixture of equal volumes of glacial acetic acid and water to $3 \mathrm{cc}$. of alcoholic solution. Under these circumstances stearic acid would be completely separated. (See $\$ \$ 16,131$.

$\S 131$. The separation of resins from fat-acids in soap-analysis has formed the subject of communications from Jean, ${ }^{2}$ Barfoed $^{3}$ and Gladding. ${ }^{4}$ The following particulars are taken from Barfoed :

a. Stearic and palmitic acids are soluble in hot 70 per cent. spirit, but separate out on standing twenty-four hours in a cool place, Coniferous resin (abietic acid) dissolves in 10 parts of cold spirit of the same strength, but is precipitated on adding water containing hydrochloric acid.

b. If a mixture of the same fat-acids with resin is boiled with 7 volumes of 30 per cent. spirit, to which 1 volume of an aqueous solution of carbonate of soda (1 to 3 ) has been added, both resin and fat-acid dissolve. On cooling; the soap produced from the fat-acids separates out, whilst the resinate of soda remains in solution. The fat-acids may be obtained from the precipitate by filtering off, washing with alcoholic carbonate of soda solution and decomposing with hydrochloric acid, whilst the filtrate yields the resin on treatment with an acid and shaking with ether.

c. On adding a solution of 1 part of chloride of calcium in 15 of 80 per cent. spirit to a hot solution in spirit of the same strength, and cooling, the calcium salts of both fat-acids separate out, whilst that of the resin acid remains in solution.

d. If stearic and palmitic acid and resin are dissolved in soda, the solution evaporated to dryness, powdered and extracted with a

1 Zeitschr. f. anal. Chem. xviii. 622, 1879 (Journ. Chem. Soc. xxxiv. 1011).

2 Polyt. Journ. ccvii. 1873 (Journ. Shem. Soc. xxvi. 195).

3 Zeitschr. f. anal. Chem. xiv. 20, 1875 (Journ. Chem. Soc. xxix. 771). Compare also Gottlieb, Poliz. chem. Skizzen, Leipzig, 1853 ; and Sutherland, Chem. News, 1866, 185.

${ }^{4}$ Chem. News, xlv. 159, 1882. 
mixture of 1 volume of 98 per cent. spirit to 5 of ether, the resin compound alone passes into solution.

Gladding's method depends upon the insolubility of the silver salts of fat acids in ether, in which resinate of silver dissolves both easily and abundantly. For working details of the process reference must be made to the original paper.

If oleic acid is present, the separation by $a$ and $b$ will be inaccurate, as the resin will be contaminated with oleic acid. These methods might, however, be employed to separate oleic from stearic and palmitic acid in absence of resin. If only a small quantity of oleic acid is present, the resin may be estimated by $c$. On decomposing the lime salt with an acid, a little oleic acid may be precipitated with the resin, but the former remains suspended in the liquid, whilst the latter agglutinates into lumps. After separating the resin, the oleic acid may be removed by shaking the liquid with ether.

The estimation of rẽsin in the presence of oleic acid is, however, best accomplished by $d$. The mixture must be well dried and the ether-alcohol made from anhydrous spirit and ether; 1 part by weight of oleate of soda dissolves in 935,1 of resinate of soda in 7.9 parts of ether-alcohol.

\section{CHLOROPHYLL AND ALLIED SUBSTANCES.}

\$ 132. Chlorophyll.-Notwithstanding that the chemical nature of chlorophyll is still involved in considerable obscurity, I treated it in $\S 20$ as a homogeneous body, and at the same time pointed out that the chlorophyll-granules observable under the microscope contain solid albuminous substances, starch, etc., in addition to chlorophyll.

It has been satisfactorily proved by Fremy ${ }^{1}$ and others that chlorophyll may be separated by treatment with hydrochloric acid and ether or benzene into two colouring matters, one of which, cyanophyll or phyllocyanin, is blue and soluble in ether and

${ }^{1}$ Comptes Rendus, 1. 405, 1860, Ixi. 188, 1865 ; Journ. f. prakt. Chem. lxxx vii. 319, 1862. See also Kromayer und Ludwig, Archiv d. Pharm. clvi. 164, 1861; Aé, Archiv d. Pharm. cxcii. 163, 1870; Kraus, 'Zur Kenntniss des Chlorophyllfarbstoffes,' Stuttgart, 1872; Wiesner, Chem. Centralblatt, 353, 1874; Filhol, Comptes Rendus, lxi. 371, lxxix. 612, 1874; Hartsen, Annal. der Phys. cxlvi. 158, 1874; 'Neue chemische Untersuchungen,' Forstemann, 1875; Archiv d. Pharm. [3], vii. 136, 1875. 
benzene, the other, xanthophyll or phylloxanthin, yellow and insoluble.

These two substances exist, according to Fremy, side by side in chlorophyll. In this opinion, however, he is opposed by Pringsheim and others, ${ }^{1}$ who assert that they are only products of its decomposition. Sorby, again, does not consider the existence of a chlorophyll, a phyllocyanin, or phylloxanthin of definite chemical composition to be probable, but rather anticipates in them representatives of whole series of such compounds. Which of these opinions may be correct it is impossible at the present time to decide.

Whether the green colouring matters isolated by Filhol, Sachsse, ${ }^{2}$ and others, and said to differ spectroscopically from ordinary chlorophyll, are of artificial origin, or whether they can be produced by the plant itself ; what relation probably exists between chlorophyll, 'purified chlorophyll,' or chlorophyllan and cyanophyll; between xanthophyll, Hartsen's crystalline chrysophyll and Pringsheim's hypochlorin, are questions involved in still greater obscurity.

I restrict myself, therefore, here, to stating that 'chlorophyll' can be extracted from vegetable substances by boiling alcohol after exhaustion with water; a little, however, is retained by the residue insoluble in alcohol, as benzene still extracts a green colouring matter possessing all the characters of chlorophyll. ${ }^{3}$

${ }^{1}$ Chem. Centralblatt, 299, 316, 331, 1880.

2 Ibid. 121, 1878.

3 That the chlorophyll exists in different states of combination is rendered probable by the fact that if vegetable substances are exhausted with petroleum spirit, benzene, ether, etc., in succession, each of these solvents removes chlorophyll, so that when petroleum spirit fails to dissolve more of it, appreciable quantities can still be extracted with benzene. This combination might be conceived to be simply mechanical, the protoplasm acting in a similar manner to hydrate of aluminium which, as is well-known, has the power of mechanically retaining chlorophyll. But the question may also be raised whether chlorophyll, which, in the opinion of many authors, possesses the characters of a weak acid, does not exist in plants in combination with different bases, and whether soluble (basic) alkali-compounds, such as those artificially produced by Frenn, do not occur ready-formed in some plants. Every one that has been frequently engaged in plant-analyses must have observed that well-filtered aqueous extracts of leaves, etc., when acidified and shaken with benzene or ether, yield to those solvents substances which on evaporation assume a green tinge and possess all the characteristic properties of chlorophyll. The assumption of the presence in the aqueous extract of a colourless chromogene converted during the successive operations into chlorophyll would, it is true, be possible, but I cannot as yet regard the first view as untenable. The whole subject, indeed, appears to me deserving of further investigation. 
After acidulating the alcoholic extract with hydrochloric acid and diluting with a little water, the chlorophyll may be removed by shaking with benzene, xanthophyll remaining in the alcoholic liquid. Under these circumstances, however, the chlorophyll is unfortunately always accompanied by fatty matter, etc.

\$133. Estimation of Chlorophyll. - Should it appear desirable to isolate the chlorophyll for the purpose of weighing (cf. $§ 37$ ), advantage might possibly be taken of an observation made by Sachsse, ${ }^{1}$ viz., that a benzene solution of chlorophyll, on standing for a few days over metallic sodium, deposits a green mass capable of being filtered off from the golden-yellow solution. With the exception of its containing sodium, it agrees with chlorophyll in most of its more important characters, although, of course, it no longer represents that substance in an unaltered state. It dissolves in water, but is completely precipitated by sulphate of copper. The copper compound thus formed may, however, be contaminated with carbonate. From it the colouring matter may be isolated by suspending in alcohol, passing a current of sulphuretted hydrogen through the mixture, and evaporating the alcoholic filtrate. The residue may be weighed.

\$ 134. Erythrophyll, Chlorophyllan, etc.-By first freeing grass from wax by treatment with ether, and then exhausting with alcohol, Hoppe-Seyler ${ }^{2}$ succeeded in isolating from unaltered chlorophyll a greenish-white colouring matter, sparingly soluble in alcohol, crystallizing in four-sided plates, and appearing red by transmitted light. This substance seems to be identical with Bougarel's ${ }^{3}$ erythrophyll. Hoppe-Seyler also separated a second substance, which was more easily soluble in hot alcohol, crystallized in needles, and appeared dark green by reflected, but brown by transmitted, light. This body, which he terms chlorophyllan, agrees with the so-called chlorophyll in most of its properties, especially the spectrum, in which, however, the bands in the yellow and green are somewhat deeper than they are in the ordinary chlorophyll-spectrum (\$ 148 and 20). Hoppe-Seyler thinks it possible to make approximate estimations of chlorophyll by titration with spectroscopic end-reaction. ${ }^{4}$ Gautier has also

${ }^{1}$ Chem. Centralblatt, 121, 1878 ; 741, 1880.

2 Ber. d. d. chem. Ges. xii. 1555, 1879 ; xiii. 1244, 1880 (Journ. Chem. Soc. xxxviii. 53,894$)$.

${ }^{3}$ Bulletin de la Soc. Chim. xxvii. 442, 1879 (Journ. Chem. Soc. xxxii. 790).

${ }^{4}$ For the chlorophyll contained in certain Florideæ, see Pringsheim, loc. cit.; 
isolated from the leaves of dicotyledonous plants a crystalline chlorophyll, ${ }^{1}$ which Hoppe-Seyler suspects to be a mixture of erythrophyll, chlorophyllan and wax. Gautier's analyses agree tolerably well with those of Hoppe-Seyler's chlorophyllan.

$\S 135$. Xanthophyll (phylloxanthin), the yellow colouring matter to which the autumnal tint of many leaves is ascribed, appears to be insoluble in water, sparingly soluble in cold ether, petroleun spirit, or benzene. Alcohol dissolves it more readily, and it is soluble also in ether-alcohol. It may be obtained as a yellow granular deposit contaminated with fatty matter by evaporating an alcoholic extract (Berzelius). Dilute acid and dilute potash and ammonia are said to dissolve it but sparingly; the latter may, therefore, be employed to effect a partial separation from fat, etc. Sulphuric and hydrochloric acids colour it only faintly blue. If the alcoholic extract has been shaken with benzene, as directed in $\S 132$, the residue obtained on evaporating the benzene solution may be purified by suitable treatment with the foregoing liquids, especially petroleum spirit. ${ }^{2}$ Hartsen thinks that his chrysophyll is possibly identical with phylloxanthin.

Hypochlorin.-Pringsheim ${ }^{3}$ states that hypochlorin separates from the chlorophyll granules in the form of yellow drops, which gradually become crystalline. It is insoluble in water, dilute acids and solutions of salts, but is easily dissolved by ether, benzene, bisulphide of carbon and ethereal oils. In concentrated and dilute alcohol it is at one time easily, at another difficultly, soluble. Possibly it is volatile with the vapour of water.

It would be premature, on the basis of the facts that have as yet been established, to assert the identity of hypochlorin with xanthophyll ; the latter is certainly not identical with etiolin, the yellow colouring matter of etiolated plants, which in alcoholic solution assumes a green tinge, and, after the lapse of some time, is coloured blue by hydrochloric acid.

for the colouring matter of certain Algæ, see Sachsse, 'Chem. und Phys. d. Farbstoffe, Kohlehydrate und Proteinsubstanzen,' Leipzig, 1877.

${ }^{1}$ Bulletin de la Soc. Chim. xxviii. 147, 1879 (Journ. Chem. Soc. xxxviii. 266).

2 For the relation that xanthophyll (etiolin) bears to chlorophyll, see Wiesner, Annal. d. Phys. und Chem. cliii. 622, 1874, and Chem. Centralblatt, 353, 1874; also 'Die Enstehung d. Chlorophyll's in der Pflanze,' Wien, Hölder, 1874.

${ }^{3}$ Chem. Centralblatt, $9,27,299,316,331,1880$. Compare also Jahresb. f. Wissensch. Bot. 1874. See Quarterly Journ. Mic. Soc. 1881. 
Anthoxanthin, the yellow colouring matter in the petals of many flowers, also differs from xanthophyll. It occurs in two varieties, one of which (anthochlor, xantheïn) is soluble in water, whilst the other (xanthin, luteïn) is dissolved only by ether and alcohol. The latter turns green and blue on the addition of hydrochloric acid.

ETHEREAL OILS, VOLATILE ACIDS, ETC.

$\S 136$. Estimation. - The following estimations are taken from Osse, and given here in illustration of the method recommended in $\$ 22$ :

I. 0.277 gram of oil of turpentine was diluted with petroleum spirit to $10 \mathrm{cc} . ; 1 \mathrm{cc}$. of the solution was evaporated as described in $\$ 22$. The weight of the residue was 0.046 gram, which, after exposure to the air for 1 minute, decreased to 0.026 gram (difference, 0.02 ) ; after a second minute's exposure, 0.0205 gram (difference, 0.0055); after a third, 0.017 gram (difference, 0.0035); after a fourth, 0.0135 (difference, 0.0035). The weight of the turpentine taken is calculated from the third weighing, 0.0205 gram, to which is added $2 \times 0.0035$ gram, making a total of 0.0275 gram from 1 cc., or 0.275 gram from 10 cc., instead of 0.277 gram. A second estimation gave 0.267 gram; mean 0.271 gram.

II. $0 \cdot 1268$ gram of oil of lemon was diluted to $5 \mathrm{cc}$. with petroleum spirit, and $1 \mathrm{cc}$. taken for evaporation.

$$
\begin{aligned}
& \text { 1st weighing }=0.0505 \\
& \text { 2nd ", }=0.0250 \text { diff. }=0.0255 . \\
& \text { 3rd ", }=0.0185, \quad=0.0065 . \\
& \text { 4th }, \quad=0.0165, \quad=0.002 . \\
& \text { 5th }, \quad=0.0145, \quad=0.002 .
\end{aligned}
$$

To the third weighing, 0.0185 gram, there is to be added $2 \times 0.002$ gram, giving a total of 0.0225 gram from 1 cc., or 0.1125 gram from 5 cc., instead of 0.1268 gram. A repetition of the estimation gave 0.1275 gram; mean 0.1200 gram instead of $0 \cdot 1268$ gram.

III. $0 \cdot 166$ gram of oil of cinnamon diluted to $10 \mathrm{cc}$; $1 \mathrm{cc}$. taken for evaporation.

$$
\begin{aligned}
& \text { 1st weighing }=0.0317 \\
& \text { 2nd ", }=0.0171 \text { diff. }=0.0146 . \\
& \text { 3rd }, \quad=0.0163, \quad=0.0008 . \\
& \text { 4th }, \quad=0.0160,=0.0003 . \\
& \text { 5th }, \quad=0.0157,=0.0003 .
\end{aligned}
$$

The third weighing, 0.0163 , represents the quantity of oil present, since no correction has to be made, as the co-efficient of 
evaporation is less than 0.001 . 10 cc. would, therefore, contain 0.163 gram instead of 0.166 gram.

$\S 137$. Estimation with Bisulphide of Carbon.-Instead of petroleum spirit, Osse also tried bisulphide of carbon, as recommended by Hager ${ }^{1}$ for the quantitative estimation of camphor, as well as mixtures of both liquids, without attaining better results. $\mathrm{He}$ has therefore decided in favour of petroleum spirit alone, which, however, should not contain any oils boiling at a temperature higher than $40^{\circ} \mathrm{C}$.

In analyzing vegetable substances such a petroleum spirit is preferable to mixtures of the same with bisulphide of carbon, as it has a lesser solvent power for resins, etc. Ethereal oils may be extracted from their aqueous solutions by petroleum spirit, ${ }^{2}$ and may therefore be estimated in the aqueous portion of the distillate ( $(24)$ by shaking with that solvent and evaporating a measured quantity of the solution after separation from the aqueous liquid. I have also employed this method for estimating the essential oil in the official aromatic waters.

$\$ 138$. Influence of Fixed Oil.-O Osse also made experiments with the view of ascertaining whether the presence of fixed oil could affect the determination of ethereal oil, either by itself increasing in weight during the exposure to the air or by preventing the evaporation of the ethereal oil at $110^{\circ} \mathrm{C}$. He found that a pretty close approximation to the truth might generally be arrived at by deducting 0.09 to 0.1 per cent. from the weight of the fat after heating to $110^{\circ}$. No appreciable error would be caused by the oxidation of the fixed oil during the evaporation of the petroleum spirit, as the presence of the latter, even in small quantities, prevents or delays such change.

0.875 gram olive oil was mixed with 0.051 gram oil of turpentine and heated for an hour to $110^{\circ} \mathrm{C}$. The weight of the residue was $0.875 \mathrm{gram}$, which did not alter if the heating were continued two hours longer.

1.4265 gram olive oil and 0.0575 gram oil of cinnamon weighed after

$$
\begin{aligned}
& 1 \text { hour at } 110^{\circ} \text {. . } 1.436 \text { gram. } \\
& 2 \text {, , . . . } 144335 \text { " } \\
& 3 \text {, , . . . } 1.4315 \text { " }
\end{aligned}
$$

${ }^{1}$ Pharm. Centralblatt, xiii. 449.

${ }^{2}$ Dragendorff, paper read at a meeting of the German 'Apothekerverein' in Cologne, 1873 ; 'Ermittelung der Gifte,' 2nd ed., 46, 1876. 
I obtained similar results in experiments with cacao butter. Resin could be almost completely freed from ethereal oil at $100^{\circ}$ to $110^{\circ}$, and it was only in the case of oils prone to uxidation, such as oil of cloves, that the residual resin was somewhat heavier than was expected. (See also $\$ 146$. )

Drying oils would, of course, increase very appreciably in weight. The evaporation and heating would have to be conducted in an atmosphere of carbonic acid ( $\$ 9)$.

The following experiment will serve as an example of the estimation of ethereal oil in a vegetable substance $:^{1}$

Five grams of savin leaves were finely powdered and digested with 25 cc. of petroleum spirit; 1 cc. of the solution was evaporated. The residue weighed 0.0265 gram (corr.), which decreased to 0.0175 gram on heating to $110^{\circ}$. 1 cc. contained, therefore, 0.009 gram ethereal oil and 0.0175 gram resin, or 4.5 per cent. of ethereal oil and 8.75 per cent. of resin.

\$139. Separation of Volatile Acids.-Angelic acid melts at $45^{\circ}$ and boils at $185^{\circ}$; methyl-crotonic acid at $65^{\circ}$ and $198^{\circ}$; crotonic acid, $16^{\circ}$ and $1605^{\circ}$; capric, $30^{\circ}$ and $268^{\circ}$ to $270^{\circ}$; caprylic, $16^{\circ}$ to $165^{\circ}$ and $236^{\circ}$ to $237^{\circ}$; cenanthic boils at $223^{\circ}$ to $224^{\circ}$; caproic, $204^{\circ}$ to $206^{\circ}$; valerianic at $175^{\circ}$; trimethyl acetic, $163.7^{\circ}$ to $163.8^{\circ}$ (melts at $35.3^{\circ}$ to $35.5^{\circ}$ ); butyric at $163^{\circ}$; isobutyric, $154^{\circ}$; propionic, $140^{\circ}$; acetic, $118^{\circ}$ (solidifies at $16.7^{\circ}$ ); formic, $105^{\circ}$. This difference in the boiling points of fat-acids permits of their separation from one another by fractional distillation.

Fractional precipitation by salts of silver, etc., may also be found useful in separating several of the foregoing volatile acids from one another; certain differences in the solubility of the salts can also sometimes be turned to account. Isobutyric acid, for instance, may be separated by the former method, whilst the sparing solubility of the silver salt ( 1 in 100) enables us to isolate acrylic, butyric, acetic acid, etc. The barium, calcium, and lead salts of some of the acids may be similarly employed; thus the barium salt of caprylic acid is soluble in 164 parts of cold water $;^{2}$ formate of calcium is insoluble in absolute alcohol ; the lead salt dissolves in 65 parts of water, whilst mercurous formate requires 500 parts at the ordinary temperature. Basic formate of lead obtainerl

1 See Osse's work previously referred to.

2 For the estimation of valerianic acid, see Zavatti and Sestini, Zeitschr. f. anal. Chemie, viii. 388, 1869. 
by heating formic acid with oxide of lead is insoluble in alcohol, ${ }_{1}^{1}$ whilst basic acetate of lead prepared in a similar way is soluble (the heating should be continued until the reaction is alkaline, but not longer, as otherwise an acetate insoluble in alcohol might be produced). Basic butyrate of lead is also soluble in alcohol, but both the neutral and basic salt are greasy and sparingly soluble in cold water. The same is the case with the ferric compound obtained by precipitating an alkaline butyrate with a ferric salt (avoiding an excess). (See also $\$ 34$.)

$\$ 140$. Identification.-The saturating power of a fatty acid, a knowledge of which may be of assistance in identifying it, can be ascertained by titration with normal soda solution, or by estimating the sodium, barium, lead or silver contained in the corresponding salts. In certain cases a determination of the water of crystallization may prove useful.

By distilling the sodium salts with concentrated sulphuric acid and absolute alcohol, the ethyl-salts of the acids may be prepared ; they are not unfrequently of characteristic odour (acetate, butyrate, valerianate of ethyl, etc.), by which, as also by their boiling points, they may sometimes be identified.

$\S 141$. Optical Tests ; Solubility in Alcohol.-For information with regard to the optical testing of volatile oils see Buignet, ${ }^{2}$ Franck, ${ }^{3}$ Flückiger, ${ }^{4}$ and Symes. ${ }^{5}$

I have ascertained that alcohol must possess the following strengths to be miscible with certain ethereal oils in every proportion : oil of turpentine, 96 per cent. ; fir, 96 per cent. ; juniper, 95 per cent. ; savin, 92 per cent. ; lemons, 97 to 98 per cent. ; bergamot, 88 per cent. ; bitter orange, 98 per cent. : caraway, 88 per cent. ; peppermint, 86 to 87 per cent. ; oleum menthæ crispæ, 86 per cent. ; lavender, 88 per cent. ; rosemary, 82 per cent. ; sweet marjoram, 82 per cent. ; cajeput, 91 per cent. ; sage, 85 per cent. ; cloves, 74 per cent. ; cinnamon, 78 per cent. ; cubebs, 90 per cent. ; fennel, 93 per cent. ; anise and rose, 93 to 94 per cent. ; balm, 90

1 ' Barfoed, Lehrbuch der organischen qual. Analyse,' Kopenhagen, 1880.

2 Journ. de Pharm. et de Chim. [3], xl. 252, 1862 (Amer. Journ. Pharm. xxxiv. 140).

3 N. Jahrb. f. Pharm. xxvii. 131; xxix. 28. See also Mierzinski, 'Die Fabrik. äth. Oele,' Berlin, 1872, and Fliickiger's 'Pharm. Chemie,' Berlin, 1879 , where the specific gravities of certain ethereal oils will also be found.

4 Archiv d. Pharm. [3], x. 193, 1877 (Amer. Journ. Pharm. Ixxvii. 309).

' Pharm. Journ. and Trans. [3], x. 207. 
per cent. These figures are true for fresh oils only, and for temperatures ranging from $20^{\circ}$ to $22^{\circ}$. 1

I found the following proportions of weaker alcohol necessary to form clear mixtures with the foregoing oils :

\begin{tabular}{|c|c|c|c|c|c|c|c|}
\hline Oil o & Cinnamon. & - & $\begin{array}{l}\text { Vols. } \\
.3\end{array}$ & of & $\begin{array}{r}\mathrm{st} \\
65 \mathrm{p}\end{array}$ & $\begin{array}{l}\text { ength of Sp } \\
\text { r cent. ('T }\end{array}$ & $\begin{array}{l}\text { pirit. } \\
\text { ralles). }\end{array}$ \\
\hline , & Cloves & • & . 27 & , & 60 & $"$ & , \\
\hline , , & Sage . & - & . $3 \cdot 1$ & $"$ & 65 & 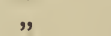 & , \\
\hline , & Cajeput . & . & . $2 \cdot 5$ & $"$ & 65 & $"$ & , \\
\hline , & Marjoram . & - & . $1 \cdot 45$ & $"$ & 78 & $"$ & 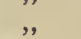 \\
\hline , & Rosemary . & • & . 1.4 & $"$ & 78 & " & , , \\
\hline, & Lavender & . & . $2 \cdot 3$ & , & 65 & " & , \\
\hline , & Mentha crispa & . & $.2 \cdot 7$ & $"$ & 65 & " & , \\
\hline , & Peppermint & . & . $2 \cdot 2$ & , & 70 & , & , \\
\hline ," & Caraway . & . & .0 .8 & , & 84 & 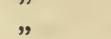 & , \\
\hline ", & Bitter orange & . & .0 .9 & $"$ & 94 & , & ", \\
\hline, & Bergamot. & . & $.1 \cdot 15$ & $"$ & 78 & , & , \\
\hline , & Lemon (dist.) & & $4 \cdot 0$ & $"$ & 91 & , & , \\
\hline , & , (pressed & & $2 \cdot 8$ & , & 92 & 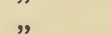 & ," \\
\hline , & Savin & 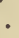 & $1 \cdot 3$ & $"$ & 80 & , & ", \\
\hline , & Juniper & • & $.3 \cdot 0$ & $"$ & 93 & , & ", \\
\hline ," & Turpentine & . & $.3 \cdot 75$ & , & 92 & , & ", \\
\hline , & Fennel & & $.2 \cdot 9$ & ", & $85(a$ & $\left.21^{\circ} \mathrm{C}.\right)$ & \\
\hline , , & Anise & . & $.6 \cdot 3$ & , & $85(a$ & $17 \cdot 5^{\circ} \mathrm{C}$. & \\
\hline
\end{tabular}

$\S 142$. Colour-reactions. - I have observed the following colourreactions with certain ethereal oils : ${ }^{2}$

Solution of bromine in chloroform ( 1 in 20 ), in the proportion of 10 to 15 drops to one of oil, gives colourless mixtures with oils of turpentine, caraway, lemon, coriander and cardamoms; yellow with bergamot, bitter orange and neroli ; slouly turning green with cloves, ginger, lavender, cajeput, cascarilla ; slowly turning greenishblue with ol. menth. crisp., oils of juniper, pepper and galangal ; greenish-brown or broun with sweet marjoram, dill, cummin and valerian; a more or less fine rose, red, or reddish-violet tint is gradually produced by rosemary, fennel, anise, star-anise, cinnamon, nutmeg, thyme, peppermint, myrrh and parsley ; brownishviolet with mace; blue or bluish-violet with cubebs, copaiba, amomum, laurel, sandal-wood and sweet flag; orange with oil of worm-seed, oil of cedar-wood; and with camphor.

1 N. Repert. f. Pharm. xxii. 1, 1872; Pharm. Journ. and Trans. [3], vi. 541 et ser. See also Godeffroy und Ledermann, Zeitschr. d. allgem. oesterr. Apotheker Ver. xv. 381 et seq. ; Jahresb. f. Pharm. 394, 1877.

2 Pharm. Journ. and Trans. [3], vi. 681; Archiv d. Pharm. [3], xii. 289. See also Hager, Pharm. Centralblatt, 137, 169, 195, 1870 ; and Fliickige:, Schweiz. Wochenschr. f. Pharm. 261, 1870. 
Impure Chloral Hydrate ${ }^{1}$ ( 2 drops to 1 of oil), resembles the foregoing reagent in the colouration it produces with many oils. It differs, however, in its behaviour to oil of lemon and bergamot, with which it assumes a reddish colour; cloves, which turns red on warming ; mace (fine rose-red), pepper (reddish-violet), copaiba (dark-green), valerian (greenish), cummin (fine green), cinnamon (green, with violet margin), and myrrh (reddish-violet).

Alcoholic hydrochloric acid varies in its action with the amount of acid it contains. A dilute solution is to be preferred, as the colourations appear more slowly, but are purer. Dilute alcoholic hydrochloric acid in the proportion of 15 to 20 drops to 1 of oil yields colourless mixtures with oil of turpentine, caraway, coriander, cardamoms (conc. acid, cherry-red), cloves, rosemary (conc. acid, deep cherry-red); yellow mixtures with bergamot (conc. acid, orange to olive-green), mace (conc. acid, reddish-brown), dill (conc. acid, cherry-red), bitter orange, cummin (conc. acid, deep violet); brounish-red with oils of cascarilla, lavender, sweet marjoram, worm-seed, juniper (conc. acid, red); rose to deep red or reddish-violet with oils of cubebs, pepper, copaiba, cedar wood, cinnamon, nutmeg, thyme, laurel, sweet-flag and myrrh ; red, turning blue, with oil of peppermint.

Concentrated sulphuric acid (2 or 3 drops to 1 of oil) assumes with most oils a yellow colour, turning brown, and frequently passing finally to a fine red. The latter colouration is observable with oils of caraway, mentha crispa, sweet marjoram, star-anise, mace, dill, juniper, cubebs, copaiba, sage, winter-green, lavender, amomum, cascarilla, nutmeg, thyme, sandal-wood, peppermint, myrrh, and parsley. Oils of cardamoms, cloves, fennel, anise, cajeput and laurel produce a violet, cinnamon a green and blue colouration.

If a drop of the oil is mixed with 1 cc. of chloroform and 2 drops of conc. sulphuric acid added, similar colours are produced ${ }^{2}$ and imparted to the chloroform.

${ }^{1}$ Jehn was the first to observe that this reagent produced a currant-red colour with oil of peppermint. Its use is, however, open to objection, as it is not yet known what impurity causes the colouration, and it is therefore impossible to prepare a reagent of constant composition. If $100 \mathrm{cc}$. of alcohol are saturated with chlorine, mixed with sulphuric acid (after partially separating the hydrochloric acid by evaporation) and the resulting metachloral distilled, a very satisfactory reagent will be obtained, but its activity diminishes on keeping.

2 But not if petroleum spirit is used instead of chloroform. 
Fröhde's Reagent ${ }^{1}$ resembles sulphuric acid in its action ; but the colours are purer and make their appearance more rapidly. Very characteristic colours are produced with some oils by sulphuric acid mixed with $\frac{1}{6}$ th of its volume of a 5 per cent. aqueous solution of ferric chloride.

The oils should be dissolved in chloroform, in which the colouring matter is also soluble and thus seen to advantage.

Oils of pennyroyal, parsley, coriander, fennel, anise, savin and turpentine cause no colouration in the chloroform, even after the lapse of some time; with oils of ledum and peppermint it assumes a red tinge; with ledum-camphor, and oils of thyme, cajeput, galangal, pepper, cubebs, copaiba, juniper, violet or bluish-violet; with serpyllum, sweet marjoram, rosemary, caraway, dill, nutmeg, cloves, worm-seed, cinnamon, green or bluish-green; with oil of bergamot, etc., olive-green.

Fuming nitric acid (5 drops to 1 of oil) gives specially characteristic colourations with oils of mace and nutmeg (blood-red), cubebs (green), copaiba (bluish-violet), gaultheria (cherry-red), cinnamon (carmine), myrrh (reddish-violet), pimento (blood-red), and pennyroyal (violet).

Picric acid (0.05 gram to 5 to 6 drops of oil) is easily dissolved by some oils in the cold (caraway, cardamoms, cloves, rosemary, mentha crispa, sweet marjoram, anise, star-anise, dill, valerian, cummin, gaultheria, cinnamon, sweet flag); by others only on warming. Some of the solutions deposit crystals on standing (turpentine, lemon, bergamot, sweet marjoram, mace, dill, galangal, bitter orange, worm-seed, valerian, cedar-wood, lavender, cajeput, nutmeg, thyme, laurel and sandal-wood); others gradually assume characteristic colourations: thus oil of mentha crispa becomes olive-green ; cloves, sweet marjoram, anise, star-anise, nutmeg, cinnamon, cummin, amomum and thyme, orange; fennel and myrrh, blood-red ; dill, cascarilla and galangal, brown ; worm-seed, recldish-brown; sweet-flag, deep brown; peppermint, deep grass green.

Flückiger ${ }^{2}$ recommends acting upon a solution of the ethereal oil in bisulphide of carbon ( 1 in 15) with sulphuric and nitric acids. With oil of valerian and nitric acid (specific gravity, 1.2) he observed a green colouration of the bisulphide, and red of the acid

11 cc. conc. sulphuric acid with 0.01 gram molybdate of soda.

${ }^{2}$ Schweiz. Wochenschr. f. Pharm. 261, 1870. 
layer; with a mixture of both acids, blue. Gurjun-balsam oil behaved similarly, and oil of cubebs also turned blue with a mixture of both acids.

Solid iodine added to ethereal oils produces somewhat varying effects. With some oils, especially terpenes of the formula $\mathrm{C}_{10} \mathrm{H}_{16}$, the action is very energetic, and accompanied by evolution of both light and heat, whilst with others nothing of the kind is observable. Chromic acid also reacts explosively with certain oils. Some oxygenated oils (carvol of cummin oil) yield crystalline sulphhydrates when mixed with alcoholic solution of sulphide of ammonium, from which the oil may be separated by decomposition with potash. ${ }^{1}$ If hydrochloric acid gas is passed through ethereal oils, crystalline or liquid hydrochlorates are not unfrequently produced, which may be characteristic of the oil acted upon. The $\mathrm{NOCl}$ group sometimes combines with hydrocarbons of the terpene series to form compounds of the formula $\mathrm{C}_{10} \mathrm{H}_{16} \mathrm{NOCl}$, and, according to Tilden, this reaction also may be employed in dis. tinguishing ethereal oils. Tilden ${ }^{2}$ obtained crystalline compounds. with French and American oil of turpentine, with oil of juniper, sage, caraway, bitter orange, bergamot, and lemon.

For the use of cohesion figures in identifying the various. ethereal oils see Kate Crane ${ }^{3}$ and Tomlinson. ${ }^{4}$

\$ 143. Fractional distillation.-Linnemann's apparatus ${ }^{5}$ (fig. 6) is very serviceable in fractionally distilling ethereal oils $(\$ 30)$. $A$ is a tube of about $40 \mathrm{~cm}$. in length and $1 \mathrm{~cm}$. in diameter; at about $32 \mathrm{~cm}$. from one end a second tube, $\mathrm{B}$, is fused on at an angle of about $80^{\circ}$, so that it can be connected with a condenser. Just beneath the junction, and at a distance of 20 and $25 \mathrm{~cm}$. from the end, bulbs are blown. At the upper end a thermometer is introduced, the bulb of which should be in C. In the lower part of the tube about 8 cup-shaped pieces of platinum gauze are inserted. These are intended to receive the condensing vapour from the liquids of higher boiling points and wash, as it were, the vapour of more easily volatile liquids. Smaller apparatuses of 30 or $25 \mathrm{~cm}$. in height may be used for special purposes.

For distillation in a partiul vacuum the apparatus represented in

${ }^{1}$ Compare Jahresb. f. Pharm. 468, 1867.

2 Pharm. Journ. and Trans. [3], viii. 188.

${ }^{3}$ Pharm. Journ. and Trans. [3], v. 242.

4 Ibid. v. 280.

${ }_{5}^{5}$ Annal. d. Chem. und Pharm, clx. 195, 1872. 
fig. 7 has been recommended by Thörner. ${ }^{1}$ The method of using it is sufficiently intelligible from the figure, and requires no special description.

\$144. Further Examination of Ethereal Oils.-For details of the analysis of ethereal oils by fractional distillation, I refer to the examination of eucalyptus oil by Faust and Homeyer, ${ }^{2}$ of parsley

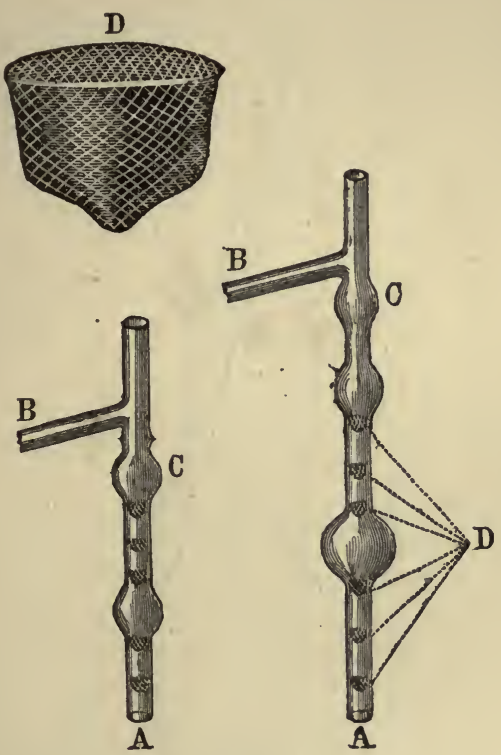

Fig. 6 .

oil by Gerichten, ${ }^{3}$ and oil of sage by Muir and Suguira. ${ }^{4}$ In the investigation of oil of dill by Nietzky, ${ }^{5}$ of oil of valerian by Bruylants (see below) and of arnica by Sigel, ${ }^{6}$ the fatty acids present in the oil are included. Ethereal salts were found by

${ }^{1}$ Ber. d. d. chem. Ges. ix. 1868, 1876. See also Bevan, Chem. News, xxxviii. $183,1879$.

${ }^{2}$ Ber. d. d. chem. Ges. vii. 63 and 1429, 1874 (Journ. Chem. Soc. xxvii. $475)$.

${ }^{3}$ Ber. d. d. chem. Ges. ix. $25 S$ and 1477,1876 (Journ. Chem. Soc. xxx. 78.)

+ Pharm. Journ. and Trans. [3], vii. 265, 1876; viii. 191, 1877.

${ }^{5}$ Archiv d. Pharm. [3], iv. 317, 1874 (Journ. Chem. Soc. xxvii. 892).

${ }^{6}$ Annal d. Chem. und Pharm. clxx. 345, 1873 (Journ. Chem. Soc. xxvii. $377)$. 
Renesse ${ }^{1}$ in the oil of Pastinaca sativa and by Möslinger ${ }^{2}$ in that of Heracleum sphondylium.

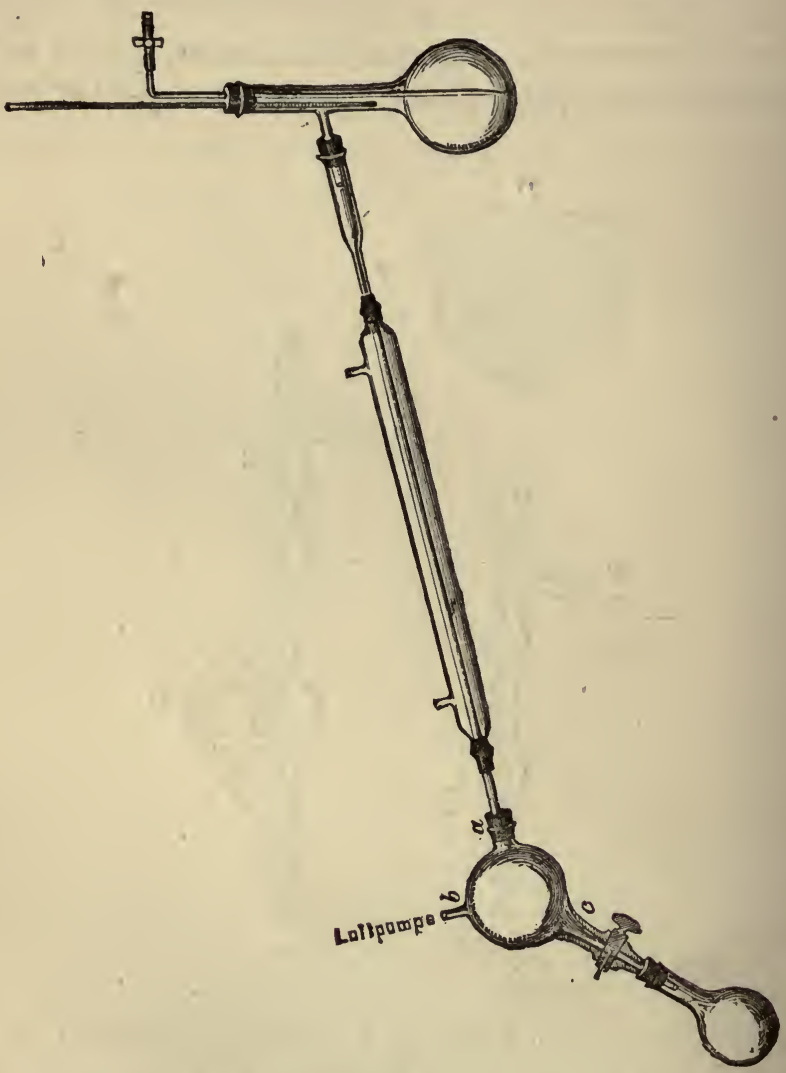

Fig. 7 .

Bruylants included aldehydal substances in his examination of oil of tansy. ${ }^{3}$

${ }^{1}$ Annal. d. Chem. und Pharm. clxi. 80 ; clxxi. 380 (Journ. Chem. Soc. xxvi. 642 ; xxvii. 1145).

2 Ber. d. d. chem. Ges. ix. 998. See also Zincke, Annal. d. Chem. und Pharm. clii. 1, 1869 ; Ber. d. d. chem. Ges. iv. 822, 1872. See also Gutzeit, 'Ueber das Vorkommen des Aethylalkohols im Pflanzenreiche,' Jena, Dufft, 1875 (Journ. Chem. Soc. xxviii. 1245).

${ }^{3}$ Ber. d. d. chem. Ges. xi. 449, 1878 (Journ. Chem. Soc. xxxiv. 157). 
RESINS, ANTHRAQUINONE-DERIVATIVES, BITTER PRINCIPLES, ETC.

$\$ 145$. Resin-acids and the more important methods for their separution.

With regard to the coniferous resin-acids, the observations made in $\$ 131$ may be supplemented by the following:

Abietic acid ${ }^{1}$ occurs in lamellar crystals, softening at $129^{\circ}$ and melting at $144^{\circ}$, soluble in alcohol and ether, and forming salts with most bases. Prolonged heating converts it into its anhydride, which is soluble in absolute alcohol, and was formerly known as pinic acid. The alcoholic solution of this substance yields no crystals on evaporation; it is gradually reconverted into abietic acid by the continued action of 70 per cent. alcohol.

Pimaric acid, from Pinus pinaster, forms granular crystalline masses melting at $149^{\circ}$, difficultly soluble in cold, but easily in boiling alcohol, and soluble in ether. It resembles abietic acid in most of its properties, but differs in possessing a bitter taste.

For podocarpic acid see Oudemans $;^{2}$ gardenin, Stenhouse and Groves ; ${ }^{3}$ phyllic acid, Bougarel. ${ }^{4}$

In isolating resin-acids one of the following methods will be frequently found successful :

a. Successive treatment with spirit of different strengths, finally adding water and shaking with ether. It will be observed that resin acids are, as a rule, more easily soluble in dilute spirit than resin-anhydrides, wax, etc. It was by this method that I succeeded in isolating mongumic acid from a bark imported from Madagascar. ${ }^{5}$ The residue obtained on evaporating the ethereal extract was treated with 85 per cent. spirit, which left a wax undissolved. The spirituous solution was evaporated, and the

${ }^{1}$ Maly considered the acid formerly known as sylvic acid to be abietic; Duvernoy regards it as a modification of pimaric acid.

2 Ber. d. d. chem. Ges. vi. 1122 ; Annal. d. Chem. und Pharm. clxx. 213 (Journ. Chem. Soc. xxvii. 72).

3 Annal. d. Chem. und Pharm. cc. 311 (Journ. Chem. Soc. 1878).

4 Union Pharm. xviii. 262, 1877 (Journ. Chem. Soc. xxxii. 905). A substance similar to that described under the above name is often met with in the analysis of herbaceous and leathery leaves. It is soluble in boiling alcohol, and separates from such solution, after the wax, on evaporating and cooling. It crystallizes in colourless scales, dissolves with difficulty in water and glycerin, is soluble in ether and chloroform, and also in warm potash, but precipitated by an excess of the latter.

5 Pharm. Journ. and Trans. [3], ix. 816 (1879). 
mass treated with 50 per cent. spirit, in which a little brown resin was found to be insoluble. To the alcoholic solution ether was added, and then sufficient water to cause separation. On well shaking the ether dissolved the whole of the mongumic acid, the addition of a few drops of acetic or hydrochloric acid facilitating solution. The mongumic acid was then obtained by evaporating the ethereal liquid.

b. Treatment of the mixed resins with a solution of soda or potush in dilute spirit, and recovery of the resin by the addition of acetic or hydrochloric acid and filtering, or, if very finely suspended, shaking with ether. I adopted this method in separating a resinacid from pæony-seed. ${ }^{1}$ The mixed resins were treated with boiling 85 per cent. spirit, and the liquid kept at $0^{\circ}$ for some time, to allow of the separation of a little resin anhydride that had been carried into solution. To the filtrate water was added till the spirit was reduced to a strength of 50 per cent., by which the resin was precipitated. The mass was then dissolved in a solution of soda in 50 per cent. spirit, again precipitated by the addition of acid, and finally decolourized in alcoholic solution by animal charcoal. In adopting this method the requisite strength of the spirit must be ascertained by preliminary experiments.

c. Treatment of the mixed resins with aqueous soda or potash.Any resin dissolved by the alkaline liquid may be generally recovered by acidification with acetic or hydrochloric acid. (Compare also $\$ 45) .^{2}$ It is, moreover, not unfrequently possible to obtain sparingly sqluble combinations of the resin with silver, lead, barium, calcium, etc., by adding salts of those metals to the solu. tion of resinate of soda. This method is sometimes suecessful in cases of mixtures of several resin-acids or of a resin-acid with other resinous substances soluble in solution of soda. The resins present may be separated by fractional precipitation; or it may happen that only one is precipitated by the salt used, in which case, of

1 Archiv d. Pharm. [3], ix. 426, 1879 (Journ. Chem. Soc. xxxvi. 1043).

2 Chrysin, discovered by Piccard in the buds of the poplar (Ber. d. d. chem. Ges. vi. 884 , 1873 ; Journ. Chem. Soc. xxvi. 1236) might be isolated by this method. It is precipitated yellow by acids, is somewhat sparingly soluble in ether and alcohol, and almost insoluble in petroleum, bisulphide of carbon, chloroform, and benzene. The latter, when warm, removes the so-called tectochrysin. An alcoholic solution of chrysin is coloured violet by ferricchloride, and gives with neutral acetate of lead a yellow precipitate, soluble in excess and in glacial acetic acid. 
course, the others remain in solution; or finally a mixture may be precipitated, in which, however, a separation may be effected by treatment with solvents or by decomposition with carbonic acid, etc. Hirschsohn met with a case of this description in his examination of galbanum. ${ }^{1}$ The resinous portion of the drug was digested with soda, and to the solution chloride of barium was added till no further precipitate was produced. From the dried barium precipitate boiling alcohol dissolved a rather large amount, which separated again on cooling, and contained only 1.07 per cent. of baryta. This portion must have been carried down either mechanically or in so loose a state of combination that boiling spirit sufficed to effect a decomposition into acid and base. The alcoholic solution contained a second resin-acid, which was partly precipitated on passing carbonic acid through the liquid, and partly, in masses of fibrous crystals resembling asbestos, on the subsequent addition of water. Boiling 95 per cent. alcohol extracted it from the dried precipitate. The dilute alcoholic liquid, after treatment with carbonic acid, was acidulated with hydrochloric acid, which threw down a flocculent precipitate, soluble in ammonia. In addition to these three resins a fourth had escaped precipitation with chloride of barium. It could be separated by passing a current of carbonic acid through the alkaline solution.

In fractionally precipitating with silver or lead salts attention should be directed to the percentage of the metal and the melting point of the resin acid contained in the precipitates. These two points are frequently of service in identifying acids.

d. The mixed resins may finally be separated by dissolving them in spirit and fractionally precipitating with alcoholic solution of acetate of lead.

$\S 146$. Resins and Gum-resins of Commerce.-The examination of commercial resins and gum-resins, which generally consist of ethereal oil and various resinous substances frequently accompanied by mucilage, sugar, etc., was at my suggestion undertaken and carried out by Hirschsohn. The following is an abstract of his results :

${ }^{1}$ Pharm. Zeitschr. f. Russland, p. 225 et seq., 1875 (Pharm. Journ. and Trans. [3], vii. 369 et seq.).

${ }^{2}$ Pharm. Zeitschr. f. Russland, 225 et seq., 1875 ; 1 et seq., 1877. 'Beiträge zur Chem. der wichtigeren Harze, Gummiharze und Balsame,' Diss. Dorpat, 1877. Archiv d. Pharm. [3], x. 481 et seq. ; xi. 54 et seq. ; xiii. 288 et seq. Pharm. Journ. and Trans. viii. 389 et seq. 
1. For the determination of the ethereal oil petroleum spirit may be used as a solvent. ${ }^{1}$ (See $\$ \S 9,22,23,138$.) But, as elsewhere observed, part of the resin will also be dissolved; the residue obtained by evaporating at the ordinary temperature till the weight is constant must therefore be heated to $110^{\circ}$ or $120^{\circ}$, and the percentage of ethereal oil calculated from the loss. The amount of resin dissolved by the petroleum spirit, which is thus simultaneously ascertained, may be of use in estimating the value of different varieties of a resin or in detecting adulterations (in the case of copal, the better the quality of the resin the smaller the percentage of non-volatile substances soluble in petroleum spirit). The mixture (of ethereal oil and resin) obtained by evaporating the petroleum-spirit solution frequently yields colour-reactions with the reagents mentioned in $\S 142$.

2. The residue insoluble in petroleum spirit is treated with ether and the substances dissolved estimated. It should be ascertained if ether takes up all the resin insoluble in petroleum spirit or if a further portion is removed by subsequent treatment with alcohol. Gum-resins will of course always leave a residue insoluble in ether, consisting of sugar, gum, salts, etc. The ethereal solution should be tested as to its miscibility with alcohol and the residue after evaporation for colour-reactions as mentioned in 1.

3. The estimation of substances soluble in alcohol, both in the original drug and after treatment with ether, together with the qualitative examination of the solution, may likewise yield results of some value. In the case of gum-resins sugar is one of the principal substances extracted by alcohol. (See $\$$ 70, 83 et seq.; 200 et seq.) It should be ascertained whether a turbidity is produced by adding ammonia, ether, or alcoholic solution of acetate of lead to the spirituous extract from the original resin.

4. If a gum-resin is under examination, water will remove gum ( $\$ 73$ et seq.; and 193 et seq.) and certain salts from the residue after treatment with alcohol. Note should be taken if a gum swelling, but not dissolving, in water is present. (See $§ \S 103$ and 193 et seq.)

5. Important results may also be obtained by treating the original resin with chloroform, ether, or saturated aqueous solution

I The resin should be rubbed down as fine as possible with powdered glass, and then macerated with petroleum spirit. 
of carbonate of soda. The latter may cause a colouration or take up cinnamic acid (detected by the permanganate of potash reaction $(\$ 26)$, etc.). For details of the scheme published by Hirschsohn for the identification of the more important resins and gum-resins, reference must be made to the original papers, etc., already quoted.

$\S 147$. Pceonio-fluorescin.-If agitation with ether (\$ 44 ) removes any substance from solution in caustic alkali it should be ascertained whether the same can be extracted from a solution in a carbonated alkali. It was found that pæonio-fluorescin ${ }^{1}$ could be obtained much purer by using a carbonated rather than a caustic alkali, as the latter partially decomposes it, whilst the former does not. Whether the seeds of other plants contain in their testa a body allied to, or identical with, pæonio-fluorescin, and possessing, therefore, a strong fluorescence in ethereal solution, is a matter for investigation.

Pæonio-fluorescin is sparingly soluble in chloroform, benzene, and cold water (somewhat more freely in warm), but insoluble in petroleum spirit. It is precipitated from a warm $\left(50^{\circ}\right)$ aqueous solution by gelatine, but not by acetate of lead or copper. On boiling with very dilute hydrochloric acid an intense green colour is developed, which can be extracted by agitation with ether, and changes to a reddish-violet in contact with acetate of soda. Its solution in very dilute lime-water, extremely weak ammonia, or even chalky spring-water, gradually assumes a fine red colour when exposed to the air.

$\S 148$. Anthraquinone-derivatives.-In treating the substances soluble in ether (\$\$ 36 and 46 ) with alkaline liquids, any change of colour, especially to red, should be carefully noted. If such is the case there is reason to take into consideration the possible presence of certain anthraquinone derivatives, such as chrysophanic acid, emodin, frangulic acid, alizarin, purpurin, etc. They are soluble in very dilute alkali, and are precipitated by hydrochloric acid from the deeply-coloured (generally red) solutions. It frequently happens that these bodies do not occur readyformed in the fresh substances, or in material that has been carefully dried, but are present in the form of glucosides ${ }^{2}$ (chrysophan, frangulin, ruberythric acid).

The following are some of the characteristic properties of the foregoing anthraquinone derivatives.

1 Archiv d. Pharm. [3], xiv. 412.

2 Compare my paper on Analyses of Rhubarb ; Pharm. Zeitschr. f. Russland, 
Chrysophanic acid, as obtained from rhubarb, senna, ${ }^{1}$ etc., is almost insoluble in water, but if in combination with a base it can be extracted from aqueous solution by adding a strong acid and shaking with ether. The solubility in alcohol and acetic acid varies directly with the strength of the solvent (1 cc. of 86 per cent. alcohol dissolves 0.00017 gram at $20^{\circ} ; 1$ cc. glacial acetic acid dissolves 0.00046 gram).

Chrysophanic acid is sparingly soluble in petroleum spirit, but is dissolved by benzene and chloroform, especially when warm. It can be sublimed in flat rhombic prisms, which melt at $162^{\circ}$, are yellow in colour, and strongly dichroic. It is easily dissolved by alkaline liquids, both aqueous and alcoholic, with production of a fine red colour, for particulars of the spectrum of which reference must be made to Keussler's dissertation. This colouration in contact with alkali serves as a means of detecting chrysophanic acid and allied substances microscopically, but it is preferable to employ baryta- or lime-water, as with these bases compounds are formed which are insoluble in water. )

Emodin agrees with chrysophanic acid in most of its properties, but may be distinguished by its insolubility in benzene, and greater solubility in ether and alcohol. It melts at $245^{\circ}$ to $250^{\circ}$, and crystallizes in needles from glacial acetic acid.

Erythroretin and Phcooretin may also be obtained from rhubarb ; they are both sparingly soluble in ether, freely in alcohol; the former is coloured purple-red by alkalies, the latter reddishbrown. ${ }^{2}$

Chrysarobin occurs in Goa powder $;^{3}$ it is soluble in boiling benzene, and forms a yellow solution with conc. sulphuric acid (chrysophanic acid, red). It is not dissolved by dilute potash, but with concentrated it yiela's a yellow solution with a green fluorescence. On shaking this liquid with air for some time it turns red, and then deposits chrysophanic acid after acidulation.

65, 97, 1878 (Pharm. Journ. and Trans. [3]. viii. 826), and the continuation of the paper by Greenish, Pharm. Journ. and Trans. [3], ix. 933.

1 Compare Keussler, 'Unters. d. chrysophansäureart. Subst. der Sennesblätter und der Frangulinsäure,' Diss. Dorpat, 1879, and Pharm. Zeitschr. f. Russland, 257 et seq., 1878. See also Kubly, 'Ueber das wirksame Princip und einige andere Best. d. Sennesblätter,'Diss. Dorpat, 1865, and Pharm. Zeitschr. f. Russland, 429 et seq., 1866 (Amer. Journ. Pharm. xxxvi. 374).

2 Compare Kubly, Pharm. Zeitschr. f. Russland, vi. 6ீ3, 1867.

${ }^{3}$ Compare Liebermann und Seidler, Ber. d. d. chem. Ges. xi. 1603 (Journ. Chem. Soc. xxxvi, 326). 
Frangulic acid can be obtained as an orange-red powder, consisting of small acicular (? hexagonal) crystals, which melt at $255^{\circ}$ and are not dichroic. At a temperature of $18^{\circ} 1 \mathrm{cc}$. of glacial acetic acid dissolves 0.00235 gram, 1 cc. of 96 per cent. spirit $0.018 \mathrm{gram}$. The solutions of this substance in aqueous or alcoholic alkalies are also of a fine red colour, but prove to be somewhat different from those of chrysophanic acid when examined spectroscopically. Keussler made the following observations with aqueous solutions in caustic potash, under the conditions mentioned in $\S 22$ :

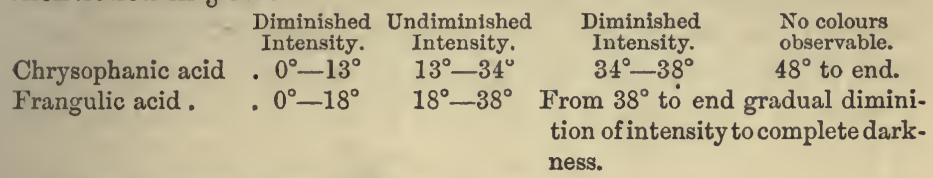

Compare Plate I., 1 and 2.1

Alizarin forms orange-red prisms, which are also almost insoluble in cold water, but soluble in alcohol, ether, benzene, and aqueous alkalies. They melt at $215^{\circ}$, and can be sublimed without decomposition. Its alkaline solutions are violet, and yield purple precipitates with salts of calcium, barium and lead. Vogel ${ }^{2}$ states that the absorption-spectrum of a solution of alizarin in dilute alcoholic potash shows two dark bands, one of which is exactly divided by the line $d$, whilst the other begins a little before $D$, and may be traced some distance past that line. (Compare Plate I., 3.) An alcoholic solution of alizarin, after addition of ammonia, shows an absorption spectrum with a single illdefined band in the green between $D$ and $F$. (Plate I., 4).

Purpurin shows under the last-named conditions two ill-defined absorption bands to the right and left of $E$ (Plate I., 5), whilst an alcoholic solution made alkaline with potash absorbs dark blue powerfully, and shows two very deep bands between $F$ and $E$, and $E$ and $D$, and one weak one at $d$. (Plate I., 6.) The difference between the spectra of alizarin and purpurin is so great that an admixture of 1 per cent. of the latter can be detected with facility in the former. The direct detection of small quantities of alizarin in purpurin is, however, impossible, but, according to

1 For frangulin and frangulic acid, see also Faust, Archiv d. Pharm. clxxxvii. 8, 1869 (Pharm. Journ. and Trans. [3], iii. 1033).

2 'Prakt. Spektralanalyse,' Nördlingen, Beck, 1877 ; and Ber. d. d. chem. Ges. x. 157. See also ibid. 175 and 550 (Journ. Chem. Soc. vol. xxxii.). 
Schunk and Römer, ${ }^{1}$ indirect proof may be obtained by taking advantage of the unequal affinity of the two substances in alkaline solution for atmospheric oxygen. A solution in caustic soda is exposed to the air until it has become almost colourless and ceases to show the spectrum of purpurin after the addition of more alkali. By acidifying with hydrochloric acid and agitating with ether, the alizarin can be extracted, redissolved in alcoholic potash, and tested spectroscopically.

The scale on Plate I. corresponds to that described in $\S 20$. I shall subsequently come to speak of the spectra of chlorophyll, hæmatoxylin, and some other colouring matters (partly taken from Vogel) also figured on the same plate.

Purpurin forms orange-red needles, melting at $253^{\circ}$, and soluble in boiling water and alcohol, but more freely so in ether, bisulphide of carbon, and boiling benzene. Aqueous solutions of alum dissolve it, forming yellow liquids with green fluorescence; with dilute aqueous alkalies purple solutions are obtained; it dissolves with difficulty in alcoholic soda, and is precipitated by lime- and baryta-water.

The erythrosclerotin, or sclererythrin, isolated by Podwissotzky and myself ${ }^{2}$ from ergot is, I think, possibly identical with, or closely allied to, purpurin.

Alizarin is generally considered to be produced from a glucoside, ruberythric acid, and not to occur ready-formed in the madder plant; ruberythric acid is possibly itself a product of the decomposition of rubian. The latter is said to be soluble in hot water and in alcohol; from aqueous solution it is not precipitated by solution of alum or lead salts, but probably it has not yet been obtained in a state of purity. Boiling solutions of alkalies dissolve rubian with production of a red colouration and formation of alizarin, rubiretin, verantin, rubiadin and sugar. Boiling dilute acids induce a similar decomposition, whilst with cold dilute alkali it yields rubianic acid.

Ruberythric acid is freely soluble in hot water, in alcohol, and in ether. It crystallizes in yellow silky prisms, and forms bloodred solutions with alkalies. Basic acetate of lead precipitates it as a vermilion-red powder. Boiling with dilute acid resolves it

${ }^{1}$ Ber. d. d. chem. Ges. x. 175, 1877 (Journ. Chem. Soc. xxxi. 664).

2 Archiv f. exper. Patholog. und Pharmakologie, vi. 154, 1876 (Pharm. Journ. and Trans. [3], vi. 1001, viii. 106). Sitz-Ber. d. Dorpater Naturf. Ges., $392,1877$. 
into sugar and alizarin. According to Stenhouse, morindin is identical with ruberythric acid, morindon with alizarin (which is doubted by Stein), and munjestin with purpurin.

With regard to the constituents of madder that have been here mentioned, and some others that accompany or can be obtained from them, I refer in particular to the investigations of Schunk, Rochleder, Stenhouse and others, for an account of which Gmelin's 'Chemistry' may be consulted.

For rhamnin, xanthorhamnin, chrysorhamnin, and their allies, see Fleury and Biswanger, ${ }^{1}$ Ortlieb, Liebermann, and Hörmann. ${ }^{2}$

Rhinacanthin, discovered by Liborius in Rhinacanthus communis, appears to possess some of the properties common to anthraquinone-derivatives. ${ }^{3}$ It occurs in the intercellular spaces in the root bark, is soluble in ether, alcohol and dilute alkali, but insoluble in pure and acidulated water. Alkalies produce a deep red colouration, which is discharged or changed to greenish by acids.

Alkannin is insoluble in water, but yields fine red solutions with ether, alcohol, bisulphide of carbon, fixed and ethereal oils. The spectrum is figured on Plate I., 11. Alkannin is uncrystallizable, dissolves in concentrated sulphuric acid (violet), in alkalies (blue), and in alcoholic ammonia.

Bixin behaves similarly to water, alcohol and ether. It dissolves in aqueous alkalies also (but the compounds thus produced are sparingly soluble in alcohol), and is coloured blue by concentrated sulphuric acid. ${ }^{4}$

Curcumin 5 is also insoluble in water, but is dissolved yellow by ether and alcohol, brown by alkalies. Boracic acid colours it red, changing to dark blue on the addition of an alkali. (For its spectrum see Plate I., 12.)

For cambogic acid, which is dissolved yellow by concentrated sulphuric acid, see Johnstone ${ }^{6}$ and Büchner. ${ }^{7}$

1 Journ. de Pharm. et de Chim. xxvii. 666 ; Repert. f. Pharm. civ. 54.

2 Bull. de la Soc. de Mulhouse, xxx. 16; Ber. d. d. chem. Ges. xi. 1618. See also Lefort und Stein, Jahresb. f. Pharm. 145, 1867 ; 127, 1868 ; 123, 1869 (Journ. Chem. Soc. xxxvi.).

${ }^{3}$ Sitz-Ber. d. Dorpater Naturf. Ges. 277, 1879 (Pharm. Journ. and Trans. [3], ix. 162).

${ }^{4}$ Compare Stein, Chem. Centralblatt, 939, 1867.

${ }^{5}$ See Linda und Daube, Journ. f. prakt. Chem. ciii. 474, and New Series, ii. 86, 1870 (Journ. Chem. Soc. xxiv. 152).

${ }^{6}$ Phil. Mag. 281, 1839.

7 Annal. d. Chem. und Pharm. xlv. 72, 1843 (Amer. Journ. Pharm. xv. 129). 
For grönhartin or taigusic acid see Stein and Arnaudson. ${ }^{1}$

Pipitzahoic acid also probably belongs to this group. ${ }^{2}$

$\S 149$. Detection of Anthraquinone-derivatives.-To prove that these substances, or others that have been separated with resins, or resins themselves, are entitled to be considered as anthracenederivatives, they may be heated dry with zinc dust in a glass tube in the same way as in ultimate analysis (i.e., a mixture of zinc dust with the substance at the end of the tube, followed by a layer of pure zinc), the products of decomposition being led into a cooled receiver., Anthracene and methylanthracene should be specially looked for; both of them are obtained in the form of crystalline sublimates. The former melts at $213^{\circ}$, possesses a blue fluorescence, is insoluble in water, sparingly soluble in alcohol, but more easily in ether, benzene and bisulphide of carbon. When dissolved in benzene it forms a compound with picric acid, which separates out in red crystals. The action of bichromate of potash and sulphuric acid converts it into anthraquinone. If anthracene alone is obtained, a derivative of that body would be indicated; methylanthracene alone or together with anthracene would arouse suspicion of the presence of a methylanthracene derivative. The latter possesses, like anthracene, a powerful blue fluorescence; it melts at $200^{\circ}$, forms with picric acid a compound crystallizing in dark red needles, yields with bichromate of potash, sulphuric and glacial acetic acids, anthraquinone-carbonic acid, which is sparingly soluble in excess of potash and melts at $278^{\circ}$. Methylanthracene is only slightly soluble in ether, alcohol and glacial acetic acid, but freely in bisulphide of carbon and benzene.

\$150. Hcematoxylin, etc.-Treatment with alkali also reveals the presence of hæmatoxylin; but it must be observed that this substance can be removed by pure or acidulated water from the evaporation-residue of the ethereal extract (\$38). ${ }^{4}$ With alkalies

J Journ. f. prakt. Chem. xcix. 1 ; Jahresb. f. Pharm. 165, I866.

2 Compare Weld, Annal. d. Chem. und Pharm. xcv. 188, 1855 (Amer. Journ. Pharm. xxx. 446).

${ }^{3}$ Compare Liebermann und Graebe, Ber d. d. chem. Ges. i. 49, 104, 1868 (Journ. Chem. Soc. xxv, 139).

4 The extraction of hæmatoxylin with ether free from alcohol and water is generally incomplete, as it is somewhat sparingly soluble in that menstruum ; a part, therefore, will probably be removed on subsequently treating with alcohol. 
hæmatoxylin produces a beautiful violet colour; it reduces alkaline copper-solution as well as salts of silver and mercury, and cannot be sublimed.

The best method of extracting hæmatoxylin from vegetable substances (such as logwood) is to macerate first with water containing a little sulphurous acid and then exhaust with ether saturated with water. (For the spectrum, see Plate I., 7 and 8.)

Brasillin resembles hæmatoxylin, and, like it, is soluble in ether, alcohol and water. Alkalies produce a carmine-red colouration, which disappears when the liquid is warmed with zinc dust, but returns on exposure to the air. The spectrum is shown on Plate I., 9. On boiling with peroxide of lead and water a strong fluorescence is developed.

Santalin is soluble in ether (yellow), and alcohol (red), but not in pure water. With dilute potash it yields a violet coloured solution, from which chloride of barium precipitates a violet barium-compound. It differs from alizarin in its melting-point $\left(104^{\circ}\right)$, in not subliming, and in yielding no anthracene. (For spectrum see Plate I., 10.)

§151. Detection and Estimation of Gallic Acid, Catechin, etc.-In addition to the foregoing substances gallic acid, catechin and pyrocatechin are extracted by water from the evaporation-residue of the ethereal extract ( $\$ 38$ ). They are deposited in acicular crystals on evaporating an aqueous solution over sulphuric acid at the ordinary temperature, or may be removed by shaking with ether, or preferably, acetic ether. If in sufficient quantity, gallic acid or catechin may be purified by re-crystallization from boiling water, the former being soluble in 3 parts of boiling and about 100 of cold water, the latter in 4 and 16,000 respectively. Heated between watch-glasses, gallic acid yields a white sublimate of pyrogallol, together with black non-volatile melangallic acid. Catechin yields pyrocatechin. (Cf. $\S \S 38$ and 42.) Conc. sulphuric acid dissolves gallic acid colourless in the cold, but on warming the liquid becomes wine-red and crimson. The addition of water now causes the separation of rufigallic acid, which is coloured transient blue by conc. potash. If only traces of the latter acid are present they may be extracted, according to Barfoed, ${ }^{1}$ from the aqueous liquid by agitation with acetic ether containing spirit, and the residue obtained on evaporation treated with potash.

\footnotetext{
${ }^{1}$ Barfoed, Lehrb. d. org. qual. Analyse. Lief. 1, 63.
} 
Under the influence of alkalies gallic acid turns rapidly green, red and reddish-brown. Like tannic acid, it yields inky mixtures with ferrous and ferric salts, but is not precipitated by gelatine from aqueous solution. It reduces nitrate of silver and alkalinecopper solution. Gallic acid is precipitated by acetate of lead, and is partially removed from aqueous solution by digestion with the hydrate of that metal. The precipitates are, however, neither quite insoluble nor of constant composition, so that they cannot be recommended as a means of estimating gallic acid except under certain conditions. If a solution of hydrate of lead in potash is boiled with very dilute solution of gallic acid, a rose or violet colour is developed, which is persistent for some time, especially in the presence of alcohol (Klunge).

Catechin colours conc. sulphuric acid, on warming, purple, changing to black. Solutions in aqueous potash, ammonia or carbonated alkalies gradually absorb oxygen and become rose-red, scarlet, passing to dark red and finally black. The alkaline solution produces at first no colouration with ferrous sulphate, but a green tinge is subsequently developed; acetate of soda is said to turn the colourless mixture instantly violet-blue, and cause the separation of a bluish-black precipitate. A very small quantity of ferric chloride colours solutions of catechin green, but excess causes decolourization and formation of a brown precipitate. Like gallic acid, catechin does not precipitate gelatine, and acts as a reducing agent. The lead salt obtained by precipitation is not suited for the quantitative determination of catechin, as it easily decomposes (turning red on exposure to the air).

A better method for estimating both catechin and gallic acid consists in agitating with ether or acetic ether and weighing the evaporation-residue, or preferably, titrating it with permanganate of potash. (Cf. $\S 52$, VII. ; $\$ 53$ and 165.)

Pyrocatechin is also easily soluble in alcohol, melts at $112^{\circ}$, and can be sublimed. Exposed to the air in alkaline solution it turns green and black; ferroso-ferric salts colour it dark green. It reduces gold and silver salts and alkaline-copper solution. With acetate of lead a precipitate is formed, which is soluble in acetic acid; solution of gelatine is not precipitated.

§ 152. Quercitrin, Quercetin, etc.-Quercitrin and quercetin, if present in the material under examination, might be partially extracted with ether ( $(36)$, by which, however, they are not very 
easily dissolved. They are both very slightly soluble in cold water; quercetin even in hot. They dissolve in the fixed and volatile alkalies, and in alcohol, crystallizing from the latter in yellow needles. Ferric chloride colours the alcoholic solutions green (quercetin red, on warming); acetate of lead produces orange-red and brick-red precipitates respectively. Both quercitrin and quercetin reduce solutions of gold and silver salts, and also alkaline-copper solution after prolonged boiling. Heating with mineral acids resolves quercitrin into isodulcite and quercetin (Löwe contradicts this, and asserts that water alone is given off). It may be extracted from aqueous solution by agitation with amylic alcohol. ${ }^{1}$ It melts at $130^{\circ}$ to $133^{\circ}$, and is insoluble in benzene, petroleum spirit, chloroform, and bisulphide of carbon.

In close relation to, but not identical with, quercitrin or rutin stands the violaquercitrin recently isolated by Mandelin ${ }^{2}$ from Viola tricolor. It was deposited in yellow acicular crystals on saturating the aqueous solution with benzene. Boiling with dilute acids decomposed it with production of glucose, quercetin, and a third body as yet not further investigated.

A body allied to quercetin appears to occur in the rhizome of Podophyllum peltatum. ${ }^{3}$ According to Podwissotzky, the other important constituents of this drug are podophyllotoxin, which melts at $115^{\circ}$ to $120^{\circ}$, is sparingly soluble in water, soluble in ether and chloroform, and precipitated from chloroformic solution by petroleum spirit; picropodophyllin, which is easily crystallizable, and soluble in 95 per cent. spirit, ether and chloroform, but insoluble in milk of lime and ammonia ; and podophyllic acid.

Gentisin is said to require 2,000 parts of cold ether for solution, and must therefore be looked for in the alcoholic extract. It forms pale yellow silky needles, which can be partially sublimed without decomposition, requires 5,000 parts of cold, 3,850 of hot water, 455 of cold and 62.5 of hot absolute alcohol for solution. Ferric

' Compare Johanson, 'Zur Kenntniss einzelner chemischer Bestandtheile der Weiden,' etc. Archiv d. Pharm. [3], xiii. 110, 1878 (Pharm. Journ. and Trans. [3], viii. 69). For Löwe's paper see Zeitschr. f. anal. Chemie, xiv. 233, 1875 (Journ. Chem. Soc. xxix. 108). See also Liebermann and Hamburger, Ber. d. d. chem. Ges. xii. 1178, 1879 (Journ. Chem. Soc. xxxvi, 944).

2 Sitz-Ber. d. Dorpater Naturforscher, Ges. 1882, p. 343.

${ }^{3}$ Compare Podwissotzky, Archiv f. Pharm. und exper. Pathologie, 29, 1880 (Pharm. Journ. and Trans. [3], xii. 217, 1011). 
salts precipitate it reddish-brown from alcoholic solution. Fused with potash it decomposes, yielding acetic acid, phloroglucin and gentisic acid. The latter is isomeric with protocatechuic acid $(\S 42)$, and is coloured deep blue by ferric chloride. An alkaline solution of gentisic acid becomes red on exposure to the air; when heated it yields hydroquinone, melting at $169^{\circ} .^{1}$

For thujin, see Rochleder and Kawalier ;2 for rutin (insoluble in ether), and robinin, Zwenger and Dronke $;^{3}$ for luteolin, Moldenhauer, ${ }^{4}$ Schützenberger, Paraf and Rochleder. ${ }^{5}$

$\S 153$. Jalapin and Allied Substances.-To the group of substances that are soluble in ether, and can be removed by dilute alkali, but not by pure water, from the evaporation-residue of the ethereal extract, there belong further some glucosidal resins $(\S 58)$, of which the jalapin of Ipomœa orizabensis may be taken as a representative.

Jalapin is readily dissolved by alcohol, and in alcoholic solution is resolved by hydrochloric acid into sugar and jalapinol; the latter is soluble in ether, but only sparingly soluble in water. The action of aqueous solution of soda converts jalapin into jalapic acid; the latter, after liberation with a strong acid, is soluble in water, but only sparingly soluble in ether.

Jalapinol appears to occur ready-formed in scammony, and possibly in scammony-root also.

Tampicin of Tampico-jalap resembles jalapin in most of its properties, but differs in composition. ${ }^{6}$ The same may be said of

${ }^{1}$ Compare Hlasiwetz and Habermann, Annal. der Chem. und Pharm. clxxv. 62 ; Ber. d. d. chem. Ges. viii. 684 (Journ. Chem. Soc. xxviii. 572). The actual gentian-bitter is not identical with gentisin. The former is easily soluble in water, is not thrown down by neutral acetate of lead, but precipitated by ammoniacal acetate and liberated from the precipitate by sulphuretted hydrogen. It can be extracted with difficulty by agitation with benzene, but with ease by chloroform; ferric chloride does not precipitate it. It is sparingly soluble in ether, and is said to dissolve in conc. sulphuric acid with red colouration, and to be decomposed by dilute sulphuric acid with production of sugar. (Compare Kromayer, loc. cit.).

2 Chem. Centralblatt, 449, 1858.

3 Ibid. 766, 1862. Annal. d. Chem. und Pharm. Supplement, i. 257 (Amer. Journ. Pharm. xxxv. 32).

${ }^{4}$ Annal. d. Chem. und Pharm. c. 180, 1856.

${ }^{5}$ Comptes Rendus, lii. 92, 1861 ; Journ. f. prakt. Chemie, xcix. 433, 1867.

${ }^{6}$ Compare Spirgatis, N. Repert. f. Pharm. xix. 452, 1870 ; Köhler und Zwicke, N. Jahrb. f. Pharm. xxxii. 1, 1869 (Pharm. Journ. and Trans. [3], i. 444). 
the convolvulin of true jalap, which is distinguished by its insolubility in ether, and of turpethin, ${ }^{\mathrm{l}}$ which is insoluble in ether, but differs in composition from convolvulin.

All these glucosidal resins dissolve in conc. sulphuric acid with purple colour.

\$154. Estimation of Santonin.-The following method may be adopted for the quantitative estimation of santonin $(\S 45)$ in worm-seed : ${ }^{3}$

15 to 20 grams of the material are digested on a water-bath for 2 hours with 15 to $20 \mathrm{cc}$. of a 10 per cent. caustic soda solution diluted with about 200 cc. of water, filtered and washed. The filtrate and washings are concentrated to 30 or 40 cc., cooled, neutralized with hydrochloric acid, and at once filtered. The precipitate is washed first with 15 to $20 \mathrm{cc}$. of water, and then with an 8 per cent. soda solution, and any crystals of santonin that may thus be separated subsequently added to the principal portion. The filtrate from the hydrochloric acid precipitate is acidified and shaken with 3 successive portions of 15 to $20 \mathrm{cc}$. of chloroform. The chloroformic solutions are washed with water and evaporated to dryness ; the residue is dissolved in the smallest possible quantity of soda, filtered if necessary (washing the insoluble portion with a very little water), and acidified with hydrochloric acid. After standing for 2 or 3 days in a cool place the santonin is filtered off, washed with 10 to $15 \mathrm{cc}$. of 8 per cent. solution of soda, dried at $110^{\circ}$, and weighed. A correction must be made of 0.002 gram for every $10 \mathrm{cc}$. of motherliquor, and 0.003 gram for every 10 cc. of washings.

Santonin can also be extracted by boiling with milk of lime. 15 to 20 grams of worm-seed are digested with 200 cc. of milk of lime diluted with 400 cc. of water on the water-bath for 6 hours, boiled for half an hour and filtered. The residue is again boiled with 10 cc. of milk of lime diluted with 200 cc. of water. The filtered decoctions and washings are evaporated to about $30 \mathrm{cc}$, excess of hydrochloric acid added, and at once filtered (the precipitate being treated with soda as before). The filtrate must stand for 5 or 6 days in a cool place, when the santonin may be collected and washed with soda-solution. The small amount that

1 N. Repert. f. Pharm. xiii. 97, 1864 (Amer. Journ. Pharm. xxxi. 374).

${ }^{2}$ Compare Archiv d. Pharm. [3], xiii. 306, 1878 (Amer. Journ. Pharm. 1. 296). 
remains dissolved in the mother-liquor and washings may be removed by shaking with chloroform.

Santonin is almost insoluble in cold water, but is dissolved by ether, alkalies and boiling alcohol. It melts at $169^{\circ}$, turns yellow on exposure to light, produces no colouration when dissolved in conc. sulphuric acid, but colours alcoholic potash transiently carmine. If a solution of santonin in sulphuric acid is heated to $150^{\circ}$, and a drop of a dilute solution of ferric chloride subsequently added, the mixture assumes a red tinge, gradually changing to violet.

$\S 155$. Picrotoxin, etc.-Amongst other substances to be looked for in the ethereal extract the following may be mentioned :

Picrotoxin.-Soluble in 150 parts of cold, and 25 of boiling water, as well as in alcohol, chloroform, and amylic alcohol. From aqueous solution ( $\$ 55)$ it may be extracted by the last two solvents, and also by ether, but not by benzene. ${ }^{1}$ It crystallizes with facility from water and alcohol in four-sided prisms, reduces alkaline copper solution, and dissolves yellow in conc. sulphuric acid. If dry picrotoxin is mixed with 6 parts of nitrate of potash, and sufficient conc. sulphuric acid to form a pasty mass, a brickred colour is developed on adding a solution of soda ( 1 to 3 ) in excess. The reaction succeeds better if the picrotoxin is moistened with nitric acid, dried on the water-bath, mixed with a very little sulphuric acid, and then with solution of soda.

Digitalin.-According to Schmiedeberg, ${ }^{2}$ this glucoside is insoluble in water and dilute soda, but soluble in warm dilute acetic acid. Alcohol, alone or mixed with chloroform, dissolves it easily, but in pure ether or chloroform it is more sparingly soluble. It is a colourless crystalline glucoside, yielding glucose and digitaliresin by decomposition with hydrochloric acid in alcoholic solution. It dissolves yellowish green in boiling hydrochloric acid, brown in sulphuric acid, the latter solution becoming violet on the addition of bromine water ( $\$ 55$ ).

Digitoxin accompanies digitalin in foxglove; it crystallizes in pearly plates and needles, is not very soluble in ether, and insoluble in water and benzene. Chloroform and hot alcohol

\footnotetext{
${ }^{1}$ See also Gaabe, 'Unters über einige Derivate des Picrotoxins.' Diss. Dorpat, 1872.

2 Archiv f. exper. Patholog. und Pharm. iii. 16, 1874 (Pharm. Journ. and Trans. [3], v. 741).
} 
dissolve it freely. Boiled with dilute acids in alcoholic solution, it is transformed into toxiresin (soluble in ether) without the simultaneous production of sugar. With hydrochloric acid it gives a reaction resembling that of digitalin, but is not coloured violet by sulphuric acid and bromine water. Digitalin, digitoxin and toxiresin are all characterized by very energetic physiological action that may be of use in their identification. ${ }^{1}$

I take this opportunity of referring to three other constituents of foxglove, which, however, are insoluble in ether. They are the following:

Digitalein.-This substance agrees in its physiological action with digitalin and digitoxin, but differs from them in its solubility in water and cold absclute alcohol. It is sparingly soluble in chloroform, and is precipitated from alcoholic solution by the addition of a large quantity of ether. Boiling with dilute acids decomposes it into glucose and digitaliresin. Sulphuric acid and bromine produce the same colouration as with digitalin. Tannic acid and basic acetate of lead precipitate it from aqueous solution (\$ 55 ).

Digitonin is, as already observed ( $\$ 79$ ), allied to saponin ; it is amorphous and soluble in water, to which it imparts the property of frothing. Ether precipitates digitonin from alcoholic solution more easily than it does digitaleinn. Baryta-water, tannic acid, and basic acetate of lead precipitate it from its concentrated aqueous solution. Boiling with hydrochloric acid resolves digitonin into glucose, digitoresin and digitonein, with a gradual development of a garnet-red colouration. Conc. sulphuric acid colours it brownish red, which is not changed to reddish violet by bromine.

Digitin is a resinous substance that can be obtained in warty crystals from alcoholic solution. It is insoluble in water, ether, benzene and chloroform, and possesses no marked physiological action.

For coriamyrtin compare Riban. ${ }^{2}$

For ericolin, which is decomposed by hot dilute sulphuric acid, yielding glucose and ericinol, see Rochleder and Schwartz. ${ }^{3}$

${ }^{1}$ Compare my ' Ermittelung d. Gifte,' 2nd ed. 272 et seq.

${ }^{2}$ Bull. de la Soc. chim. de Paris, vi. 87, 1864 ; vii. 79.1865 (Amer. Journ. Pharm. xxxvi. 114).

${ }^{3}$ Annal. d. Chem. und Pharm. lxxxiv. 366, 1852, and Chem. Centralblatt, 61, 1853. Compare also my 'Ermittelung d. Gifte,' 2nd ed., 300 et seq. 
Ericinol is characterized by its odour. (Compare also $\$ 55$ and 167.)

Vanillin (cf. § 167), the aromatic constituent of vanilla, is very sparingly soluble in cold petroleum spirit, but might be partially carried into solution in the presence of fixed or ethereal oil ; as a rule, however, it may be found in the ethereal extract. It is colourless, crystalline, possesses the pleasant odour of vanilla, and is soluble in 183 parts of water (at $18^{\circ}$ ), in 4.4 parts of alcohol (specific gravity 0.803 ), and in 6.24 parts of ether. It melts at $82^{\circ}$, and is soluble in dilute soda ; ferric chloride colours the aqueous solution dark bluish-violet. Being an aldehyde of methylprotocatechuic acid, it combines with acid sulphites (§33), and it is upon this property of vanillin that the following (Thiemann and Haarmann's) method of estimation is based :1

The ethereal extract of about 30 grams of vanilla is evaporated to $150 \mathrm{cc}$., and thoroughly shaken for 10 to 20 minutes with a mixture of water and saturated aqueous solution of bisulphite of soda. The ethereal solution is separated and again shaken with $100 \mathrm{cc}$. of the bisulphite mixture. The aqueous solutions are united and washed with pure ether to remove impurities. To every $100 \mathrm{cc}$. there are then gradually added 150 cc. of a mixture of 3 vols. of pure sulphuric acid with 5 vols. of water. The sulphurous acid evolved is received into solution of soda, the remainder being expelled by passing a current of steam through the liquid. After cooling, the vanillin, which has been liberated, is extracted by shaking with 3 to 4 successive portions of ether, and can be weighed after evaporating the ethereal solution.

Ostruthiin, which resembles vanillin in being sparingly soluble in petroleum spirit, ${ }^{2}$ is not precipitated by that liquid from ethereal solution. It crystallizes in delicate, pale yellow needles, melting at $91^{\circ}$; is insoluble in cold water, sparingly soluble in boiling water and benzene, freely in alcohol and in ether. The alcoholic solution possesses a feeble blue fluorescence, which is increased by the addition of water. With aqueous alkalies it forms strongly fluorescent solutions, from which carbonic acid precipitates the ostruthiin unaltered. It gives no characteristic reactions

1 Zeitschr. f. anal. Chemie, xv. 350, 1875 (Journ. Chem. Soc. xxix. 112).

2 Compare Gorup-Besanez, Annal. d. Chem. und Pharm. clxxxiii. 321, 1876 (Pharm. Journ. and Trans. [3], vii. 984). 
with metallic salts, nor does it yield angelic acid or allied substances when acted upon by alkalies.

Peucedanin 1 is allied to, but not identical with, ostruthiin. It yields no valerianic or angelic acid, but is decomposed by the action of acids into oreoselon and methyl-compounds; it is, in fact, dimethyl-oreoselon. Peucedanin melts at $76^{\circ}$, is colourless, crystalline, insoluble in cold water, but freely soluble in alcohol and ether.

Oreoselon can be obtained from peucedanin ; it is almost insoluble in cold water, but soluble in alcohol, ether and benzene. Bisulphide of carbon, ammonia and dilute alkalies dissolve it only when warmed, and the latter solution reduces alkaline copper-salts. The alcoholic solution is not altered by ferric chloride. A blue fluorescent solution is yielded by conc. sulphuric acid, but not by alkalies. Fused with an alkali, it yields acetic acid and resorcin (§ 42).

Athamanthin. ${ }^{2}$ - The statement that this substance is divaleryloreoselon requires further investigation. It crystallizes in colourless needles melting at $79^{\circ}$, is insoluble in water, but dissolves in diluted alcohol and in ether.

Laserpitin ${ }^{3}$ forms colourless prisms, melting at $114^{\circ}$, and is sparingly soluble in water and alkalies, but freely in alcohol, ether, chloroform, benzene, and bisulphide of carbon. Conc. sulphuric and fuming nitric acid dissolve it with red colouration; boiling with alcoholic potash is said to decompose it into angelic acid and laserol.

Cubebin can also be obtained in colourless crystals, melting at $120^{\circ}$, difficultly soluble in cold, more easily in warm water, and soluble in 26 parts of ether, 76 of cold and 10 of boiling alcohol. It can be removed from aqueous solution by shaking with benzene or chloroform. Conc. sulphuric acid is coloured red by cubebin. Aqueous alkalies do not dissolve it. ${ }^{4}$ (Cf. §55.)

Betulin is likewise tolerably freely soluble in ether and boiling

${ }^{1}$ Compare Hlasiwetz und Weidel, Annal d. Chem. und Pharm. clxxiv. 67 ; Heut, ibid. clxxvi. 70 (Journ. Chem. Soc. xxviii. 258, 772).

${ }^{2}$ Compare Schnedermann und Winkler, Annal. d. Chem. und Pharm. li. 315,1844 ; Hlasiwetz und Weidel, ibid. clxxiv. 67.

3 Compare Feldmann, 'Ueber das Laserpitin.' Diss. Göttingen.

4 For an analysis of cubebin compare Schmidt, Jahresb. f. Pharm. 51, 1870 (Pharm. Journ. and Trans. [3], ii. 270). For cubebin see Weidel, Jahresb. f. Pharm. 68, 1877 (Amer. Journ. Pharm. 1. 257). 
alcohol, but insoluble in water and petroleum spirit. 'It dissolves in conc. sulphuric acid, from which it can be precipitated by water. Betulin forms white crystals, which melt at about $200^{\circ}$, and are not attacked by aqueous alkalies. ${ }^{1}$

Anacardic acid ean be obtained as a white crystalline mass, melting at $26^{\circ}$, freely soluble in alcohol and ether, and dissolving in conc. sulphuric acid with blood-red colouration. ${ }^{2}$

Cardol is a colourless oil accompanying anacardic acid in the cashew nut. It is soluble in alcohol and in ether, but not in water, and possesses powerful vesicant properties (not shared by anacardic acid). It can be removed from suspension in water by agitation with chloroform. Contact with dilute potash for a short time does not result in the loss of the vesicant property of cardol, as is the case when the alkali is concentrated and the action prolonged. The tough mass thus produced becomes red on exposure to the air, and gives with basic acetate of lead a precipitate that shows the same peculiarity.

§ 156. Absinthiin, etc.-The following bitter principles are also soluble in ether : absinthiin ${ }^{3}$ (dissolves in conc. sulphuric acid with brown colour, passing to violet. See also § 55), adansonin, ${ }^{4}$ alchornin, ${ }^{5}$ anthemic acid, ${ }^{6}$ antirin ${ }^{7}$ aristolochia-yellow, ${ }^{8}$ arnicin, ${ }^{9}$ asclepiadin, ${ }^{10}$ beberic acid, ${ }^{11}$ cailcedrin, ${ }^{12}$ caryophyllin ${ }^{13}$ (coloured bloodred by concentrated sulphuric acid, cf. $\S 55)$, cascarillin ${ }^{14}$

1 Compare Hausmann, 'Beiträge zur Kenntniss des Betulins,' Göttingen, 1878.

2 See Städeler, Annal d. Chem. und Pharm. Ixiii. 137, 1847 (Amer. Journ. Pharm. xx. 139).

${ }^{3}$ Compare Kromayer, Archiv d. Pharm. cviii. 129, 1868.

${ }^{4}$ Compare Walz, Jahrb. f. prakt. Pharm. xxiv. 100, 242 ; xxvii. 1; Wittstein, Vierteljahressch. f. prakt. Pharm. iv. 41.

5 Compare Frenzel, Archiv d. Pharm. xxiii. 173, 1829 ; Biltz, ibid. xii. 46, 1826.

${ }^{6}$ Compare Jahresb. f. Pharm. 51, 1867 ; 46, 1871.

${ }^{7}$ Compare Walz, Jahrb. f. prakt. Pharm. xxvii. 74, 129 (Amer. Journ. Pharm. xxxv. 295).

${ }^{3}$ Compare Frickinger, Repert, f. Pharm. [3], vii. 12.

${ }^{9}$ Compare Walz, N. Jahrb. f. Pharm. xiii. 175 ; xiv. 79 ; xv. 329 , 1860, 1861 (Amer. Journ. Pharm. xxxiii. 451).

${ }^{10}$ Compare List, Annal. d. Chem. und Pharm. Ixix. 125, 1849.

11 Compare Maclagan, Annal. d. Chem. und Pharm. xlviii. 106, 1843 ; Iv. 105, 1845 (Amer. Journ. Pharm. xix. 113).

12 Compare Caventou, N. Jahrb. f. Pharm. xvi. 335, 1861.

13 Compare Bonastre, Jahrb. f. Pharm. xi. 103 ; and Jahn, Annal. d. Chem. und Pharm. xix. 333, 1837.

14 Compare Trommsdorf, N. Journ. f. Pharm. xxvi. 2, 142; and Duval, N. Jahrb. f. Pharm. viii. $95,1857$. 
(the same), chimaphilin, ${ }^{1}$ chiratin ${ }^{2}$ and ophelic acid, cicutin, ${ }^{3}$ columbin. ${ }^{4}$

Cotoï ${ }^{5}$ crystallizes in quadratic prisms, is sparingly soluble in cold water, freely in alcohol, ether and chloroform. It melts at $130^{\circ}$. Ferric chloride colours the alcoholic solution dark brown. Warming with nitric acid colours cotoin blood-red, paracotoin brown. The latter melts at $152^{\circ}$ (uncorrected). A description of leucotin, oxyleucotin and hydrocotoin will be found in the researches on cotoïn and paracotoïn above referred to.

Elaterin $^{6}$ is sparingly soluble in ether, and is coloured yellow by conc. sulphuric acid. 1 to 2 drops of carbolic acid produce a red tinge, which changes to crimson on the addition of the same quantity of conc. sulphuric acid. (See also § 55.)

I may mention further, erythrocentaurin, ${ }^{7}$ eupatorin,${ }^{8}$ guacin, ${ }^{9}$ hop-bitter. ${ }^{10}$ (To isolate the hop-bitter Isleib exhausts hops with cold water, absorbs the bitter principle with charcoal, extracts it from the same with 90 per cent. alcohol, distils, and, after separating the resin, shakes the resulting liquid with ether. He confirms the statement that hop-bitter is not a glucoside, but, on boiling with dilute acids, combines with a molecule of water yielding sparingly soluble lupuliretin. Part of the hop-resin may be removed from aqueous solution by shaking with petroleum

${ }^{1}$ Compare Fairbank, Vierteljahresschr. f. prakt. Pharm. ix. 582, 1860 (Amer. Journ. Pharm. xxxii. 256).

${ }^{2}$ Compare Kemp, Pharm. Journ. and Trans. [3], 1, 251, 1870 ; Höhn, Archiv d. Pharm. clxxxix. 229, 1869.

3 Compare Wikszemski, 'Ein Beitr. z. Kenntniss der giftigen Wirkung d. Wasserschierling.' Diss. Dorpat, 1875, and Jahresb. f. Pharm. 493, 1875.

${ }^{4}$ Compare Boedecker, Annal. d. Chem. und Pharm. 1xix. 37, 1849 (Amer. Journ. Pharm. xx. 324).

${ }^{5}$ Compare Hesse, und Jobst. Neues Repert. f. Pharm. xxv. 23, 1876 ; Ber. d. d. Chem. Ges. x. 149, 1877 ; Annal. d. Chem. und Pharm. cxcix. 17, 1879 (Pharm. Journ. Trans. [3], vi. 764 ; vii. 495, 1019 ; x. 521, 541).

${ }^{6}$ Compare Zwenger, Annal. d. Chem. und Pharm. xliii. 359, 1842 ; Walz, N. Jahrb. f. Pharm. xi. 21, 178, 1859 ; Köhler, N. Repert. f. Pharm. xviii. $577,1869$.

7 Compare Méhu, Jahresb. f. Pharm. 70, 1866 ; 92, 1870 ; 56, 1871 (Amer. Journ. Pharm. xxxviii. 303).

${ }^{8}$ Compare Righini, Journ. f. Pharm. xiv. 623.

${ }^{9}$ Compare Pettenkofer, Repert. f. Pharm. Ixxxvi. 311 ; Fauré, Jahrb. f. Pharm. xxii. 291.

${ }^{10}$ Compare Lermer, Vierteljahresschr. f. prakt. Pharm. xii. 504, 1863 ; Bissell, Amer. Journ. Pharm. xlix. 582, 1877; Griessmayer, Ber. d. d. Chem. Ges. xi. 292, 1878 (Journ. Chem. Soc. xxxiv. 449); Isleib, Archiv d. Pharm. [3], xvi. 345, 1880 (Journ. Chem. Soc. xl. 101) ; Cech, Zeitschr. f. anal. Chem. xx. 180, 1881 (Journ. Chem. Soc. xl. 946). 
spirit. Griessmayer has availed himself of this property of hopresin in the examination of beer. Cf. $\S 55$.) Other bitter principles are hurin, ${ }^{1}$ jervasic acid, ${ }^{2}$ juniperin, ${ }^{3}$ liriodendrin, ${ }^{4}$ lycopin, ${ }^{5}$ marrubin, ${ }^{6}$ mangostin, ${ }^{7}$ masopin ${ }^{8}$ and meconin. ${ }^{9}$ The last-named is soluble in hot water, and can be extracted fromaqueous solution after acidification with sulphuric acid, by shaking with benzene, chloroform, or amylic alcohol. With benzene it can be obtained fairly pure, and can be detected by conc. sulphuric acid, in which it dissolves without at first producing any colouration; but the solution gradually assumes a greenish, and in the course of twentyfour hours a reddish tinge. If the liquid is then warmed, the colour changes to emerald-green, blue and violet, and becomes finally red.

Meconin is accompanied in opium by meconic acid, which is sparingly soluble in water and ether, but more easily in alcohol. Boiling with water or dilute acids decomposes meconic acid; with ferric chloride it strikes a blood-red colour, which is not discharged by a little hydrochloric aci or chloride of gold. It can be removed from aqueous solution by shaking with amylic alcohol. Meconate of calcium is soluble, but the magnesium salt only sparingly so. Chelidonic acid from Chelidonium majus is sparingly soluble both in cold water and in alcohol. ${ }^{10}$

There may be further mentioned here, methysticin ${ }^{11}$ (which is slightly. soluble in cold ether and dissolves in pure conc. sulphuric acid with fine reddish-violet, in commercial with blood-red colouration), kawaïn, ${ }^{12}$ narthecin, ${ }^{13}$ nucin $^{14}$ (coloured purple by alkalies),

${ }^{1}$ Compare Boussingault and Rivero, Annal. de Chim. et de Phys. xxviii. 430 (Amer. Journ. Pharm. ii. 346).

${ }^{2}$ Compare Weppen, Jahresb. f. Pharm. 31, 1872 (Journ. Chem. Soc. xxvi. 906).

${ }^{3}$ Compare Steer, Wiener Acad. Anz. B. xxi. 383.

${ }^{4}$ Compare Emmet, Repert. f. Pharm. Ixxv. 88 (Amer. Journ. Pharm. iii. 5).

${ }^{5}$ Compare Geiger, Repert. f. Pharm. xv. 11.

${ }^{6}$ Compare Kromayer, Archiv d. Pharm. cviii. 257, 1862.

${ }^{7}$ Compare W. Schmidt, Annal. d. Chem. und Pharm. xciii. 83, 1854 (Amer. Journ. Pharm. xxvii. 331).

${ }^{8}$ Compare Genth, Annal. d. Chem. und Pharm. xlvi. 126, 1843.

9 Compare Pelletier, Annal. d. Chem. und Pharm. lxxxvi. 190, 1853, and Anderson, xcviii. 44, 1856. See also my 'Ermittel. d. Gifte,' 2nd ed., 238.

${ }^{10}$ See Lerch, Chem. Centralblatt, 449, 1846.

11 Compare Nölting and Kopp, Monit. scientif. [3], iv. 920, 1874 (Pharm. Journ. Trans. [3], vii. 149).

12 Ibid.

13 Compare Walz, N. Jahrb. f. Pharm. xiv. 345, 1861.

${ }^{14}$ Compare Vogel and Reinschauer, N. Repert. f. Pharm. v. 106 (1856) ; vii. 1 (1858). 
plumbagin ${ }^{1}$ (coloured cherry-red by small quantities of alkalies), polygonic acid, ${ }^{2}$ quassin ${ }^{3}$ (which is soluble in water, can be removed by shaking with benzene or chloroform. See also $\S 55)$, rottlerin ${ }^{4}$ sicopirin,${ }^{5}$ tanacetin, ${ }^{6}$ tanghinin, ${ }^{7}$ taraxacin,${ }^{8}$ xylosteï,${ }^{9}$ xanthosclerotin, or scleroxanthin. ${ }^{10}$

$\$ 157$. Lichen Acids and allied Substances.-Amongst the constituents of plants that are soluble in ether a number occurring in lichens may finally be mentioned here. Some of them possess the characters of acids, as, for instance :

Roccellic acid, which is itself insoluble in water, but forms soluble alkaline salts. It can be detected in the gonidia by alkanna. ${ }^{11}$

Others are characterized by yielding beautifully coloured compounds when acted upon by alkalies, ferric chloride or chlorinated lime, properties that would indicate some relation to orcin and allied bodies. Others, again, possess the chemical characters of ethereal salts, being resolved by alkalies into stronger acids and alcohols. To the former group belong the following:

Lecanoric acid (diorsellic acid), which is coloured deep red by chlorinated lime (avoiding an excess), and is decomposed at $153^{\circ}$ into orcin and carbonic acid. ${ }^{12}$

Orsellic acid, which undergoes a similar decomposition at $176^{\circ}$,

${ }^{1}$ Compare Dulong, Jahrb. f. Pharm. xiv. 441.

${ }^{2}$ Compare Rademacker.

${ }^{3}$ Compare Wiggers, Annal. d. Chem. und Pharm. xxi. 40, 1837 ; Goldschmidt und Weidel, Ber. d. Wiener Akad. 1xxiv. 389, 1877 (Journ. Chem. Soc. xxxiv. 80). See also my 'Ermittelung d. Gifte,' 2nd ed., 300 et. seq. ; and Jahresb. f. Pharm. 619, 1878. Also Christensen, Archiv d. Pharm. x vii. $481,1882$.

${ }^{4}$ Compare Anderson, Chem. Centralblatt, 372, 1855 (Amer. Journ. Pharm. xxxii. 325) ; Groves, Jahresb. f. Pharm. 161, 1873 (Pharm. Journ. Trans. [3], iii. 228).

${ }^{5}$ Compare Peckolt, Zeitschr. d. Oesterr. Apotheker-Ver. 289, 1876 (Pharm. Journ. Trans. [3], vii. 69).

${ }^{6}$ Compare Leroy, Journ. de Chim. med. xxi. 357 ; Leppig, 'Chem. Unters. d. Tanacetum vulgare,' Diss Dorpat, 1882.

${ }^{7}$ Compare Henry, Journ. de Pharm. x. 52 (Amer. Journ. Pharm. viii. 102).

${ }^{8}$ Compare Kromayer, 'Die Bitterstoffe,' 97 ; and Polex, Archiv d. Pharm. xix. 50, 1840.

${ }_{9}^{9}$ Compare Hübschmann, Pharm. Vierteljahresschr. v. 197 ; and Enz, ibid. $196,1856$.

${ }^{10}$ Compare Dragendorff and Podwissotzki, loc. cit.

${ }^{11}$ Compare Schunck, Annal. d. Chem. und Pharm. lxi. 66, 78 ; also Hesse, cxvii. 332, 1861 (Journ. Chem. Soc. iii. 153).

12 Schunck, Annal. d. Chem. und Pharm. xli. 157, 1842 (Journ. Chem. Soc. i. 71 ) ; liv. 261, 1845 ; lxi. 64, 1847 ; Stenhouse, ibid. lxviii. 57, 1848 ; cxxv. 353, 1863 (Journ. Chem. Soc. xx. 221) ; Hesse, cxxxix. 22, 1866. 
and with alkaline solutions at a temperature as low as the boilingpoint. ${ }^{1}$ Both this and the foregoing acid form salts of ethyl when boiled with alcohol.

Gyrophoric acid, which is sparingly soluble in ether, yields orcin on decomposition with alkali, and turns red on exposure to ammoniacal air. ${ }^{2}$

Parellic acid, which is only slowly coloured under the same conditions. $^{3}$

Ceratophyllin, which strikes a violet colour with ferric chloride and blood-red with chlorinated lime. ${ }^{4}$

Patellaric acid, which, in alkaline solution, turns red when exposed to the air. Ferric chloride colours it blue; chlorinated lime, blood-red. ${ }^{5}$

Evernic acid yields orcin by dry distillation, is coloured darkred by ammoniacal air, but only yellow by chlorinated lime. ${ }^{6}$

Everninic acid (oxyusnetinic acid?) is also coloured yellow by chlorinated lime, but does not change when exposed to ammoniacal air.

Usnic acid behaves in a similar manner, but an alkaline solution turns red on exposure to air, and the acid itself yields betaorcin by dry distrillation. ${ }^{7}$

Carbusnic acid ${ }^{8}$ (is sparingly soluble in ether) gives no colour reactions.

Vulpinic acid (chrysopicrin), which is more easily soluble in bisulphide of carbon and chloroform than in ether, is obtainable in yellow crystals and forms yellow salts with alkalies. Boiling with baryta-water resolves it into alphatoluic acid, oxalic acid, and methyl-alcohol. ${ }^{9}$ It may therefore be placed in the group of

1 Schunck, Annal. d. Chem. und Pharm. xli. 157, 1842 (Journ. Chem. Soc. i. 71) ; liv. 261, 1845 ; lxi. 64, 1847 ; Stenhouse, ibid. lxviii. 57, 1848; cxxv. 353, 1863 (Journ. Chem. Soc. xx. 221) ; Hesse, cxxxix. 22, 1866.

2 Compare Stenhouse, ibid. lxx. 218, 1849.

3 Compare Schunck, ibid. liv. 274, 1845 ; Strecker, 1xviii. 114, 1848.

${ }^{4}$ Compare Hesse, ibid. cxix. 365, 1861.

${ }^{5}$ Compare Weigelt, Journ. f. prakt. Chem. cvi. 28, 1869.

${ }^{6}$ Compare Stenhouse, ibid. Ixviii. 86, 1848 ; Hesse, ibid. xlvii. 297, 1861.

7 Compare Knop, Annal. d. Chem. und Pharm. xlix. 103, 1843 ; Rochleder and Held, ibid. xlviii. 1, 1843; Stenhouse, ibid. lxviii. 97, 114. Knop and Schnedermann, Journ. f. prakt. Chem. xxxvii. 363, 1843 ; Hesse, Annal. d. Chem. und Pharm. cxvii. 343, 1861.

${ }^{8}$ See Hesse, ibid. cxxxvii. 241, 1866 ; Ber. d. d. chem. Ges. x. 1324, 1877 (Journ. Chem. Soc. xxxii. 896).

${ }^{9}$ Stein, Chem. Centralblatt, 556, 1864; 432, 1865. See also Spiegel, Ber. d. d. chem. Ges. xiii. 1629, 1880. 
ethereal salts previously mentioned. The same is the ease with erytluric acid (sparingly soluble in ether), which is regarded as diorsellinate of erythrite, ${ }^{1}$ picroerythrin (orsellinate of erythrite), and betaerythric acid ${ }^{2}$ (orsellinate of betapicroerythrin).

For picrolichenin compare Alms, Stenhouse, and Groves ; 3 for cetraric and lichenostearic acid, Schnedermann and Knop ; ${ }^{4}$ for variolinin, Robiquet; $;^{5}$ stictic acid, Schnedermann and Knop ;6 lobaric acid, Knop $;^{7}$ atranoric acid (hydrocarbo-usnic acid ?), zeorin, sordidin, Paterno $;^{8}$ calycin, Hesse. ${ }^{9}$

Microscopical examination shows that the majority of these acids adhere in the form of minute granules to the exterior of the hyphæ, in heteromerous lichens almost exclusively in the cortical portion of the upper surface, or, in old specimens, on the margin of the thallus (Physicia parietina). ${ }^{10}$

To test for a lichen-acid yielding orcin as a product of decomposition, the substance under examination, or part of the lichen itself, may be heated with dilute potash, chloroform added, and the warming continued for some time in the water-bath. If such an acid is present, homofluorescin will be produced, and the solution will appear reddish-yellow by transmitted, and show a fine yellowish-green fluorescence by reflected light. Usnic acid is said not to give this reaction, which is yielded by lecanoric, erythric and evernic acid (by the last-named after continued boiling with milk of lime).

Erythric and lecanoric acid are extracted from the lichen by digestion with ammonia, and are precipitated by acetic acid. On warming, erythric acid passes into solution, whilst lecanoric acid remains undissolved.

${ }^{1}$ Compare Heeren, Schweiz. Journ. lix. 313; also Schunck, Stenhouse, Strecker, Hesse, already quoted.

${ }^{2}$ See Menschutkin, Bullet. de la Soc. chim. [2], ii. 424, 1864. Lamparter, Annal. d. Chem. und Pharm. cxxxiv. 243, 1865.

${ }^{3}$ Annal. d. Chem. und Pharm. i. 61, 1832 (Amer. Journ. Pharm. xvi. 262) ; ibid. clxxxv. 14, 1877 (Proc. Roy. Soc. lx. 68).

* Annal. d. chem. und Pharm. lv. 144, 159, 1845.

5 Annal. de chim. et de Phys. xlii. 236.

6 Jahresb. f. Pharm. 76, 1845.

7 Chem. Centralblatt, 173, 1872 (Journ. Chem. Soc. xxv. 639).

${ }^{8}$ Ber. d. d. chem. Ges. x. 1100 and 1382, 1877 (Journ. Chem. Soc. xxxi. 89 ; xxxii. 270).

${ }_{9}$ Ber. d. d. chem. Ges. xiii. 1816, 1880 (Pharm. Journ. Trans. [3], xi. 471).

${ }^{10}$ Compare Schwartz in Cohn's 'Beitrïge zur Biologie d. Pflanzen,' iii. Part II. and Archiv d. Pharm. [3], xix. 124, 1881. 
Usnic acid, which occurs in yellow crystals, yields a colourless ammonium salt.

$\S 158$. Orcin and Betaorcin. Estimation of Orcin.-Orcin and betaorcin, which have already been mentioned as products of the decomposition of certain constituents of lichens, and which sometimes occur ready formed in plants, can be obtained in colourless acicular crystals soluble in water, alcohol, and ether. Exposure to light colours them reddish; alkalies, chlorinated lime and ferric chloride, violet. By the action of ammonia and air orcin yields a blue colouring matter, whilst, under the same conditions, betaorcin gradually turns red. Orcin melts at $58^{\circ}$, betaorcin at a temperature above $109^{\circ}$.

Reymann estimates orcin in lichens by titrating with brominewater, ${ }^{1}$ by which monobromorcin is first produced and subsequently converted into tribromorcin. To the solution of orcin in a stoppered bottle titrated bromine-water is added till the precipitate has assumed a yellowish colour and excess of bromine is present, which is then estimated by iodide of potassium and hyposulphite of soda. The amount of orcin present is calculated from the equations :

and

$$
\mathrm{C}_{7} \mathrm{H}_{8} \mathrm{O}_{2}+\mathrm{Br}_{2}=\mathrm{HBr}+\mathrm{C}_{7} \mathrm{H}_{7} \mathrm{BrO}_{2}
$$

$$
\mathrm{C}_{7} \mathrm{H}_{7} \mathrm{BrO}_{2}+2 \mathrm{Br}_{2}=2 \mathrm{HBr}+\mathrm{C}_{7} \mathrm{H}_{5} \mathrm{Br}_{3} \mathrm{O}_{2} \text {. }
$$

\section{TANNIC ACIDS.}

$\S 159$. Constitution. - In estimating tannic acids an error has generally been committed in overlooking too completely the chemical differences existing between the various substances that have received this name. It has usually been considered sufficient to determine quantitatively the value of a reagent in terms of gallotannic acid, the tannin most easily procurable, and to apply the results thus obtained to the estimation of other tannins. This would be admissible under the assumption that all tannins possessed approximately identical equivalent weights and produced nearly identical chemical effects. But it has already been shown in $\S 52$ that such is not the case. It will be sufficient here to repeat that tannins exist which do not allow of comparisons with one another, even with regard to their constitution. At present many tannic acids may be assumed to be glucosides,

\footnotetext{
${ }^{1}$ Ber. d. d. chem. Ges. viii. 790,1875 (Journ. Chem. Soc. xxvii. 1293).
} 
splitting up under the influence of dilute acids into glucose and some second substance, but of a number it must be denied that they possess any such glucosidal characters.

$\$ 160$. Glucosidal nature.-In enumerating the characters of a newly-discovered tannic acid, it is, therefore, important to state whether it has been found to be a glucoside or not (\$61). The examination may be made by heating weighed quantities of the tannin with 1 to 2 per cent. aqueous hydrochloric acid in sealed tubes to $100^{\circ}$ for several hours, allowing them to stand for some time after being opened, in order to observe whether any sparingly soluble decomposition-product separates out in the cold. If this is the case, the substance may be filtered off, but at the same time it is advisable to ascertain whether any portion that may remain in solution cannot be removed by shaking with ether, acetic ether or chloroform. After warming to expel dissolved traces of those liquids, the solution may be examined for glucose (\$ 61, 83, et seq. ; 200, et seq.).

The decomposition-products that are thus obtained, together with glucose, are sometimes crystalline, as, for instance, gallic acid, from the tannin of galls, sumach, myrobalans, divi-divi (cf. $\S 151$ ), and the yellow ellagic acid from the tannic acid of the pomegranate and bablah fruits. But they are generally amorphous, difficultly soluble in pure or acidified water and in pure ether; soluble in water containing ammonia, and freely soluble in spirit; they are, as a rule, deep in colour, and agree in all essential properties with the phlobaphenes mentioned in $\$ \$ 48$ and 108. Some are so sparingly soluble that they may be of use in the quantitative estimation of the respective tannins. This is especially the case if, after the action of the acid, the liquid is evaporated to dryness and treated with water, when they often remain behind almost entirely insoluble.

Substances of this description are yielded by the decomposition of the tannic acid of oak, willow, elm, fir, birch, and acacia-bark, as well as by that from rhubarb, male-fern, ledum, wine, and by many others. Chemically the phlobaphenes approach many resins, with which they share the solubility in alcohol and slight solubility in water, differing from them in their solubility in dilute ammonia, but resembling them again in the substances they yield when fused with an alkali. (Cf. $§ 42$.) Lignin and suberin also appear to be connected with the phlobaphenes. 
The phlobaphenes mentioned in $\S 48$ may, as stated, have been produced from tannins during manipulation, whilst those in $\S 108$ will probably have existed ready-formed in the material under examination.

But, although phlobaphenes are insoluble in pure water, they are dissolved by solutions of tannic acid, sugar, and other substances, and small quantities may therefore have been extracted by water.

$\S 161$. Proneness to Decomposition. - The determination of the glucosidal or non-glucosidal nature of a tannin is sometimes a matter of considerable difficulty, because, on the one hand, it is not always easily separated from any glucose with which it may be contaminated, and, on the other hand, many tannins readily decompose, yielding bodies resembling their mother-substances in possessing a similar action on hide, gelatine, etc. A decomposition of this kind is observable with gallotannic acid, which, especially on prolonged heating in aqueous solution, apparently undergoes dissociation into a polygallic acid and sugar. Some tannins, too, from barks, etc., appear to be capable of parting with a portion of their glucose without completely losing their action on gelatine, etc., which, however, rapidly diminishes, even at the ordinary temperature, on allowing the aqueous solution to stand. It is not surprising, therefore, that very varying statements are met with as to the glucosidal pature of a tannin, and especially the amount of glucose certain members of the class can be made to yield. And yet it is most important to determine whether glucose can be obtained from a tannic acid or not. The production of a sparingly soluble and possibly crystalline body from an easily soluble amorphous tannin by the action of hydrochloric or sulphuric acid ( $(160)$ is insufficient proof of its glucosidal nature, since non-glucosidal tannins undergo a similar decomposition.

As a rule the action of dilute acids on a tannin results in the formation, apart from glucose, of a single decomposition-product belonging to the aromatic series (gallic acid, ellagic acid, phlobaphenes, etc.), but the researches on the tannic acids of the Nymphaceæ recently carried out in my laboratory by Grüning prove that two or more decomposition-products can be obtained from one tannin. From Nymphæa alba and Nuphar luteum non-glucosidal tannic acids were isolated, undergoing no further separation by fractional precipitation with lead, and yielding, 
when warmed with a dilute acid, gallic and ellagic acids together with a phlobaphene. ${ }^{1}$

\$162. Purification.-The ease with which tannins decompose renders their preparation in the state of purity desirable for accurate investigation a matter of considerable difficulty, and we may confidently assert that this has not been attained with the majority of the substances belonging to this class that have hitherto been described.

In preparing pure tannins the following hints may be useful, in addition to those given in $\$ \varsigma 49$ to 51 and 60 :

1. If a tannic acid is to be separated from the alcoholic extract it is very advisable, after evaporating, to mix at once with a considerable quantity of water. Alcohol dissolves phlobaphenes and resinous substances, together with tannic acid, and strong aqueous solutions of the latter are known to be capable of taking up the former, even if otherwise insoluble in water (\$160).

2. In precipitating the aqueous filtrate with acetate of lead, it is advisable to add the reagent in successive portions, rejecting the first (generally more deeply coloured) and last precipitates, as they are usually contaminated with foreign substances to a considerable extent.

3. The lead precipitates should be washed and treated with sulphuretted hydrogen as rapidly as possible, to avoid decomposition of the tannate of lead.

4. The filtrate from the sulphide of lead should be evaporated, if possible, in a partial vacuum, and only to the consistence of a thin syrup. The remainder of the water may be evaporated over sulphuric acid and lime at the ordinary temperature, the operation being completed in vacuo.

It will frequently be found advantageous to shake the filtrate, before evaporating, with ether or acetic ether, which would remove any gallic acid that might be present.

Many tannins may be purified by dissolving in water, adding chloride of sodium, and removing the tannic acid by shaking with acetic ether or a similar solvent. This method has been successfully used by Loewe for sumach-tannic acid and some others, ${ }^{2}$ and by Raabe for rhatania-tannic acid. ${ }^{3}$ It should be

I Beiträge zur Chemie der Nymphaceen, Diss. Dorpat, 1881.

2 Zeitschr. f. anal. Chem. xii. 128 ; xiv. 35, 44 (Journ. Chem. Soc. xxvii. 171 ; xxviii. 75).

${ }^{3}$ Pharm. Zeitschr. f. Russland, 577, 1880. 
observed that gallic acid must be removed by shaking with ether before chloride of sodium is added, and that certain tannins are partially precipitated by saturating their aqueous solutions with salt; some also can be precipitated from their aqueous solutions by sulphuric or other mineral acid, but this method seldom yields them in a sufficient state of purity for our purpose.

$\S 163$. Tannins insoluble in Water.-The tannic acids of alder ${ }^{1}$ and hop, ${ }^{2}$ together with some others, are stated to be insoluble in water after isolation. In some instances the tannin may possibly have been partially decomposed during the process of isolation (\$§ 48, 161).' In any case in which tannins sparingly soluble in water are anticipated, the lead precipitate should be decomposed in the presence of spirit.

There are also a number of bodies which resemble one or other of the tannins in certain of their properties (as, for instance, in being precipitated by acetate of lead), but are insoluble in cold water. Pæniofluorescin might be placed in this class. ${ }^{3}$

$\S 164$. It not unfrequently occurs that a single plant contains two or more tannins : for example, in addition to their own peculiar tannins, both oak and willow bark contain a little gallotannic acid ; myrobalans and divi-divi contain gallotannic and ellagotannic acid. ${ }^{5}$ If the presence of more than one tannin is anticipated, the method of fractional precipitation should be tried, or, if that is unsuccessful, the examination of the products obtained by warming with acids may afford the information required, as was the case in the investigation of oak and willow bark; gallic acid can be removed by shaking with ether, whereas oak-red cannot.

$\S 165$. Notes on some of the more important Tannins. - In the following notes those tannins will be mentioned first that are not glucosides, and yield principally pyrocatechin $(\S 43)$ by destructive distillation.

Catechu-tannic acid is probably produced from catechin (catechuic acid) by loss of the elements of water. ${ }^{6}$ In estimating catechu-

${ }^{1}$ Compare Reichardt, Chem. Centralblatt, N. F. i. 12.

${ }^{2}$ See Etti, Polyt. Journ. cclxxxviii. 354, 1878 (Journ. Chem. Soc. xxxiv. 797) ; Bissell, Amer. Journ. Pharm. [4], xlix. 582, 1877.

$3 \S 84$ and 147.

${ }^{4}$ Compare Johanson, loc. cit.

${ }^{5}$ Compare Loewe, Zeitschr. f. anal. Chem. xiv. 35, 44, 1875.

6 Compare Etti, Annal. d. Chem. und Pharm. clxxxvi. 327, 1878 ; Zeitschr. f. anal. Chem. xii. 285,1873 ; xiii. 113,1874 ; Journ. f. prakt. Chem. cv. 32. 
tannic acid good results can be obtained by using a 1 per cent. solution of gelatine in saturated solution of chloride of ammonium as indicated in $\$ 52$, xii. ; chloride of ammonium should be added to the tannin solution also. ${ }^{1}$ Lehmann has shown that the liquid may be diluted within certain limits without affecting the result to any notable extent, and that it is advisable to promote the subsidence of the precipitate by adding powdered glass and vigorously stirring. He determines the end of the experiment by removing a drop with a filtering tube and testing it with solution of gelatine on a watch-glass with dark background. The tannin solution should be mixed with an equal volume of saturated chloride of ammonium solution. Each cc. of the reagent indicates 0.0139 gram catechu-tannic acid. No other constituents of catechu are precipitated by gelatine.

Catechuic acid (§151), which is easily converted into catechutannic acid, should not be neglected in determining the value of a catechu. Lehmann endeavoured to estimate it from the difference in the amount of permanganate of potassium required (cf. $\S 52$, vii.) before and after precipitation with gelatine (by which catechu-tannic acid alone is removed). The results he obtained were, however, somewhat too high, since an infusion of catechu contains other substances besides catechuic and catechutannic acids that act upon permanganate of potassium. A more successful process consisted in removing the catechuic acid by shaking with ether, as directed in $\S 151$, and then titrating it with permanganate of potassium, reckoning 4.84 parts of catechuic acid for every 16 parts of oxygen consumed.

Rhatania-tannic acid, like the two preceding substances, yields phloroglicin and protocatechuic acid when fused with potash. ${ }^{2}$ For this tannin also Günther recommends the estimation with solution of gelatine, calculating 0.01302 to 0.01323 gram rhatania.tannic acid for every cc. of gelatine solution. The lead precipitate, which is tolerably stable but not quite insoluble in water, contains, according to Günther, 31.26 per cent. of oxide of lead.

${ }^{1}$ Lehmann, 'Vergl. Unters. einiger Catechu und Gambier-Proben,' Diss. Dorpat, 41, 1880; Pharm. Zeitschr. f. Russland, No. 18, 1881.

${ }^{2}$ Compare Raabe, loc. cit. Raabe contests the glucosidal character of rhatania-tannic acid, and is of opinion that it simply loses water when converted intn rhatania-red. See also Chem. Centralblatt, xii. 467, 1867 ; Annal. d. Chem. und Pharm. cxliii. 274, 1867, in which Grabowski, like Wittstein, still maintains the production of glucose in the decomposition. 
Raabe found 33.4 per cent. of oxide of lead in the lead-salt, and 16.64 of oxide of copper in the copper-salt. ${ }^{1}$

Morintannic acid, which occurs together with morin and maclurin in fustic, also yields phloroglucin and protocatechuic acid by fusion with potash. Boiling water extracts morin from the wood in the form of a calcium-compound, which is sparingly soluble in cold water, and is deposited, therefore, from the decoction on cooling. Alcohol acidified with sulphuric acid decomposes the compound, dissolving the morin ; the latter crystallizes from alcoholic solution in yellow needles, which are sparingly soluble in cold, but more freely in boiling water. With acetate of lead the boiling alcoholic solution gives an orange-red precipitate containing 58.4 per cent. of oxide of lead.

According to Loewe, an aqueous solution yields both morintannic acid and maclurin to acetic ether. By dissolving the evaporation-residue in cold water and adding chloride of sodium an amorphous precipitate of the former is obtained, whilst the latter crystallizes out on standing. Maclurin is insoluble in a mixture of equal volumes of water and saturated solution of salt, whereas morintannic acid is dissolved. Morintannate of lead contains $64 \cdot 23$ per cent. of oxide of lead. No accurate method of estimation is known.

The doubt that has recently been thrown upon the glucosidal nature of rhatania-tannic acid renders it very desirable that kino-, tormentil- and bistort-tannic acids should be examined afresh. ${ }^{2}$ These tannins yield similar products when fused with potash. Kino-tannic acid is characterized by the disposition its alcoholic solution shows to gelatinize. According to Günther both kinoand tormentil-tannic acids can be estimated by gelatine-solution (see above, and also $\$ 52$, xii.), 1 cc. of which indicates 0.0130 gram kino, and 0.0168 gram tormentil-tannic acid.

For ellago-tannic acid see below.

The tannin of the horse-chestnut, ${ }^{3}$ which is likewise non-glucosidal,

${ }^{1}$ Possibly there is another copper-salt containing 22 to 23 per cent. $\mathrm{CuO}$; that would, at least, appear probable from some experiments of Guinther.

2 For the tannin of kino see Eisfeldt, Annal. d. Chem. und Pharm. xcii. 101, 1854 ; for its crystalline decomposition-product, kinoin, see Etti, Ber. d. d. chem. Ges. xi. 1879 (Journ. Chem. Soc. xxxvi. 159). Tormentil-tannic acid is discussed by Rembold, Annal. d. Chem. und Pharm. cxlv. 5, 1868 (Amer. Journ. Pharm. xl. 311).

${ }^{3}$ Compare Chem. Centralblatt, x. 318 ; xii. 513. 
is partially precipitated from aqueous solution by chloride of soclium, and by acid sulphite of potassium. The aqueous or alcoholic solution turns dark cherry-red when warmed with hydrochloric or sulphuric acid, and deposits red flocks ; bichromate of potassium produces a dark colouration and brown precipitate; with ferric chloride it strikes a green, or, if the solution is ammoniacal, a violet colour; it is not precipitated by tartar emetic. No method is known by which it can be accurately estimated.

Amongst the glucosidal tannins those may be mentioned first which, when boiled with dilute acids, yield, in addition to glucose, crystal-line decomposition-products. The most important of them is-

Gallotannic acid, the decomposition-product of which, gallic acid, has been already alluded to (\$151). Its quantitative estimation is comparatively easy, as fairly accurate results can be obtained both volumetrically by titration with gelatine-solution or with permanganate of potassium, and gravimetrically by precipitation as tin (stannous), copper, or lead-salt. A few sources of error must, however, be here alluded to. In the first place, if the tannic acid has been extracted by water, mucilage and gallic acid may also have passed into solution; the latter is precipitable by gelatine in the presence of mucilage. That is avoided by extracting with spirit. In the second place, gallic acid, as already pointed out, acts upon permanganate of potassium. In titrating with a solution of that substance, therefore, either the gallic acid must be removed by shaking with ether, or, as suggested by Löwenthal, and mentioned in $\$ \S 52,53$, two estimations must be made, the one before, the other after, precipitating the tannic acid with gelatine, the calculations being made from the difference. In precipitating with acetate of lead or copper (but not with ammonio-chloride of tin), gallic acid is also partially carried down, and should therefore be previously removed. From a solution containing about 2 per cent. of tannin the tin precipitate will contain $19 \cdot 77$ to 19.79 per cent. of stannous oxide, the lead 50.00 per cent. of oxide of lead, and the copper 38.28 per cent. of oxide of copper.

Hammer's method ( $\$ 52, x i$.$) may, as already stated, be best$ applied to the estimation of gallo-tannic acid.

The tannins of sumach, ${ }^{1}$ knoppern galls, valonia and algaro-

1 Compare Günther, 'Beitr. zur Kenntn. der im Sumach, etc., vork. Gerbs.,' Diss. Dorpat, 1871 (Journ. Chem. Soc. xxiv. 762) ; Loewe, Zeitschr. f. anal. 
billa ${ }^{1}$ correspond exactly to gallotannic acid, and all that has been said of the latter is equally true of the former. They are always accompanied by gallic acid in the materials that yield them.

Some of these also contain the so-called ellago-tannic acid, which is found in notable quantities in myrobalans, divi-divi and bablah fruits. ${ }^{2}$

This ellago-tannic acid, which, as far as Loewe's experiments show, is not a glucoside, differs from gallotannic acid in yielding ellagic in the place of gallic acid, a change that can be, brought about by water alone at a temperature of $108^{\circ}$ to $110^{\circ}$. Ellagic acid can be obtained in sulphur-yellow crystals, which are almost insoluble in boiling water or in ether, and sparingly soluble in alcohol. Notwithstanding, however, its slight solubility in ether, small quantities can be removed from aqueous solution by shaking with that liquid. Ferric chloride produces first a green, then an inky colouration. It is soluble in potash, and is precipitated by acetate of lead from an alcoholic solution in the form of leadsalt, containing 63 per cent. of oxide. The dry substance heated with zinc dust yields the hydrocarbon ellagene $\left(\mathrm{C}_{14} \mathrm{H}_{10}\right)$, which cannot be combined with picric acid.

Whether ellago-tannic acid has been prepared in a state of purity, and whether it is identical with punico-tannic acid, ${ }^{3}$ are questions which we may for the present leave out of consideration. According to Rembold, the latter also yields ellagic acid. Special methods for the estimation of these two substances have not as yet been published.

For nymphæa-tannic acid see $\S 161$.

Gallotannic and gallic acids also occur in tea, accompanied by quercetin (possibly present in sumach also, $\S 152$ ), and by the socalled boheic acid. ${ }^{4}$ The latter is not thrown down when acetate of lead is added to a hot infusion of tea, but is precipitated on

Chem. xii. 128, 1873 (Journ. Chem. Soc. xxvi. 748); xiv. 46 (tannin of knoppern-galls).

1 Compare Godeffroy, Zeitschr. d. Oesterr. Apoth.-Ver. 132, 1879 (Year-book Pharm. 215, 1879).

${ }^{2}$ Compare Gïnther, loc. cit. Also my observations in the Jahresbericht $\mathrm{f}$. Pharm. 192, 1875 ; and Loewe, Zeitschr. f. anal. Chem. xii. 128 ; xiv. $35,44$.

3 Annal. d. Chem. und Pharm. cxliii. 285, 1867. I may observe that in the pomegranate bark also the substance yielding ellagic acid is accompanied by gallotannic acid, and that Rembold obtained sugar by the decomposition of the former.

* Compare Hlasiwetz, Annal. d. Chem. und Pharm. cxlii. 233. 
adding ammonia. It is pale yellow, amorphous, and easily soluble in alcohol.

Caffea-tannic ${ }^{1}$ acid yields sugar and crystalline caffeic acid. Caffeic acid is easily soluble in alcohol, sparingly in cold water, and strikes a dark green colour with ferric chloride, which is turnerl red by soda. It reduces silver salts on warming, but not alkaline copper solution. Like caffea-tannic acid, caffeic acid yields pyrocatcchin by dry distillation. The former is also coloured grreen by ferric chloride. Its ammoniacal solution turns green when exposed to the air (viridic acid). According to Günther's experiments, it cannot be quantitatively determined by precipitation with copper, lead, or gelatine. Titration with permanganate of potassium might possibly yield approximate results.

The following tannins are provisionally considered by many chemists to be glucosides (see note); they yield amorphous decomposition-products resembling phlobaphenes. (Cf. § 160.)

Quercitannic acid, which is probably identical with the tannic acids of willow and elm bark, 2 is one of the less stable tannins, and is, therefore, extremely difficult to purify and to estimate (\$ 161). The colper and lead salts seem specially liable to be decomposed by the combined action of air and water, whilst even the tannic acid itself in aqueous solution. rapidly undergoes change. To have any value, therefore, estimations by gelatine or permanganate of potassium must be made with perfectly fresh infusions. But the mucilaginous and other substances that are simultaneously dissolved by water from the oak-bark, also act upon the reagents and render the estimation inaccurate. By extracting with alcohol such foreign substances are excluded; but the estimation cannot be madc in alcoholic solution, and distillation can scarcely be effected without partial decomposition of the tannin.

${ }^{1}$ See Hlasiwetz, Annal. d. Chem. und Pharm. cxlii. 220, 1867 (Amer. Journ. xxxviii. 504); also Mulder und Olaanderen, Jahresb. f. Chem. 261, 1858.

${ }^{2}$ Compare E. Johanson, 'Beitr. zur Chem. d. Eichen-, Weiden-, und Ulmenrinde,' Diss. Dorpat, 1875 ; Grabowski, Annal. d. Chem. und Pharm. cxlv. 1, 1868. For oak-red see also Böttinger, ibid. ccii. 269, 1850. Loewe has recently contested the glucosidal nature of quercitannic acid. Compare Zeitschr. f. anal. Chem. xx. 208, 1881 (Journ. Chem. Soc. xl. 901). Loewe believes cak-red to be a kind of anhydride produced from the tannic acid by loss of 4 or 3 molecules of water. Böttinger (Ber. d. d. Chem. Ges. xiv. 2390 ; Journ. Chem. Soc. xl. 1041) considers quercitannic acid itself to be a glucoside, and points out the difference between that substance and another constituent of nak-bark also soluble in water and capable of tanning. The latter was the body isolated by Loewe. 
The best results would probably be obtained by extracting directly with alcohol, evaporating the tincture in a partial vacuum, treating the residue with water, quickly filtering and estimating at once with gelatine or permanganate of potassium. (Cf. $§ 551,52$, VII. and XII.) In standardizing the solutions, it may be useful to remember that, according to Günther's experiments, quercitannic acid, though differing greatly in other respects from gallotannic acid, possesses the same quantitative action on permanganate of potassium. It must be observed that tannic acid is deposited when its solution is completely saturated with chloride of ammonium; it is advisable, therefore, when precipitating with gelatine, to follow the directions given for titrating catechu-tannic acid. Quercitannic acid is sparingly soluble in ether; ferroso-ferric salts produce inky mixtures with its aqueous solution; other of its properties are mentioned in $\S \S 49,51$. The lead salt obtained by precipitation with a slight excess of the acetate contains 56 to 57 per cent. of oxide, the copper salt 29.5 per cent. The oak-red produced artificially from the tannic acid is identical with the phlobaphene that occurs naturally in the bark. It is likewise coloured black by iron salts, yields protocatechuic acid and phloroglucin when fused with potash, and possesses the properties of a phlobaphene as enumerated in $\$ 108,160$.

The tannins of the pine, lirch, many species of acacia, etc., which have been but little investigated, may possibly resemble quercitannic acid in many of their essential characters.

Filix-tannic acid ${ }^{2}$ is resolved, on boiling with acids, into glucose and red flocks of filix-red; the latter closely resembles cinchonared.

Cinchona-tannic acid ${ }^{3}$ undergoes a similar decomposition with production of cinchona-red. Its lead salt is somewhat easily soluble in acetic acid.

${ }^{1}$ Compare Kawalier, Wiener Akad. Ber. xi. 354 et seq. ; Rochleder und Kawalier, ibid. xxix. 22 et seq. ; Wittstein Vierteljahresschr. f. pract. Pharm. iii. $14,1854$.

2 See Malin, Chem. Centralblatt, xii. 468, 1867. For tannaspidic acid and pteritannic acid, the former of which Malin believes to be impure filix-red, see Luck, ibid. 657, 676, 1851. Compare further Grabowski, Annal. d. Chem. und Pharm. cxlii. 279, 1867.

${ }^{3}$ Compare Rembold, Annal. d. Chem. und Pharm. cxliii. 270, 186\%, and Schwarz, Chem. Centralblatt, 193, 1852. 
Cinchona-nova-tannic aci $l^{1}$ yields, according to Hlasiwetz, under: the same conditions, sugar and cinchona-nova-red; the latter is easily soluble in ether.

For ipecacuanha-tannic acid ${ }^{2}$, see Willigh and Podwissotzki; for leditannic acid, Willigh ${ }^{3}$ and Rochleder and Schwarz; ${ }^{4}$ for nucitannic acid, Phipson $;^{5}$ for the tannin of mate, Arata $;^{6}$ for celastrus-tannic acid, Dragendorff. ${ }^{7}$ Information concerning some other tannins may be gained from Gmelin's 'Chemistry.'

\section{OTHER GLUCOSIDES.}

\$ 166. Cyclopin, Rhinanthin, etc.-Cyclopin, which, however, cannot, without some consideration, be classed with the tannins, is a glucosidal substance found by Greenish, ${ }^{8}$ in the so-called Cape or Bush tea. It is freely soluble in water, and is precipitated from solution by acetate of lead, as well as by digestion with the oxyhydrate of that metal ; from the combinations with lead thus obtained, it can be liberated by sulphuretted hydrogen. Ether precipitates it from alcoholic solution. Boiled with 4 per cent. hydrochloric acid, cyclopin decomposes into glucose and cyclopiared, which latter is insoluble in ether. With strong hydrochloric acid, the solution turns rapidly red. Cyclopin is not precipitated by gelatine or tartar emetic, and does not possess an astringent taste. In the plant producing it, it appears to be easily converted into oxycyclopin, which is insoluble in alcohol, and undergoes a similar decomposition to cyclopin itself.

Another glucoside that yields a deeply coloured decompositionproduct when boiled even with very dilute acids, is the rhinanthin, occurring in various species of Rhinanthus, Alectorolophus, and Melampyrum. ${ }^{9}$ It can be obtained in colourless acicular crystals, soluble in water and alcohol, insoluble in ether, and not preci-

1 See Hlasiwetz, Annal. d. Chem. und Pharm. lxxix. 130, 1857.

2 Journ. f. pract. Chem. li. 404 ; Pharm. Zeitschr. f. Russland, xix. 1. Pharm. Journ. Trans. [3], x. 642.

${ }^{3}$ Chem. Centralblatt, $790,1852$.

${ }_{4}^{4}$ Zeitschr.f. anal. Chem. v. 668, 1869.

5 Ibid. p. 812.

G Jahresb. f. Pharm. 164, 1878. Compare also Byasson, ibid.

7 Archiv d. Pharm. [3], xii. 113, 1878.

${ }^{8}$ Sitzb. d. Dorpater Naturforscher-Ges. 345, 1880 (Pharm. Journ. and Trans. [3], xi. 549). It is accompanied by the crystalline cyclopia-fluorescin, which is soluble in ether and alcohol but sparingly soluble in water. Potash dissolves it with yellow colour and production of a fine green fluorescence.

${ }^{9}$ Compare Iudwig, Archiv d. Pharm. cxlii. 199, 1870. 
pitated by acetate of lead. Boiled with dilute!hydrochloric acid it yields rhinanthogenin, which is of a dark bluish-green colour, and insoluble in water.

Some alkaloids, too, possess the property of yielding, under similar conditions, deeply coloured decomposition-products, as for instance, rhœadine, thebaine (\$189).

$\$$ 167. Other important Glucosides; Sulubility.-A remarkable peculiarity of the above, as well as a number of other glucosides, is that, although more or less easily dissolved by alcohol, they are sparingly or not at all soluble in ether. Certain glucosides that have been mentioned, where necessary, in the foregoing chapters show a similar insolubility in ether (compare convolvulin, $\S 153$; digitaleïn and digitonin, $§ 155$; chrysophan, $§ 148$, etc.); in fact, this negative property may be said to be characteristic of the majority of glucosides.

Nitrogen enters into the composition of some few of the members of this class, and certain of them, when acted upon by ferments or acids, yield in addition to sugar a volatile decomposition-product of characteristic odour; but this is not the case with most of them.

The following are some of the better-known glucosides that are soluble in alcohol, contain nitrogen, and yield a volatile decompositionproduct.

Amygdalin and laurocerasin ${ }^{1}$ are both tolerably easily soluble in water (amygdalin in 12 parts), and in boiling alcohol of sp. gr. 0.819 , but more sparingly in cold. They are insoluble in petroleum spirit, and are precipitated by ether from alcoholic solution. Amygdalin crystallizes with facility in bitter scales belonging to the monoclinic system. Laurocerasin has not yet been obtained in crystals. They are both lævo-rotatory. Conc. sulphuric acid dissolves them with pale reddish-vioiet colour. Emulsin easily resolves them into glucose, oil of bitter almonds, and hydrocyanic acid, the latter body being produced in larger quantity from amygdalin than from laurocerasin. The reason for this is to be found in the fact that in laurocerasin half of the cyanogen in the amygdalin group has been already converted into formic acid, so that laurocerasin may be regarcied as amygdalate of amygdalin. On boiling amygdalin and laurocerasin, therefore, with baryta-water, the former will yield one molecule of ammonia for every molecule of

${ }^{1}$ Compare Lehmann, 'Ueber das Amygdalin der Kirschen, Pflaumen,' etc., Diss. Dorpat, 1874. 
barium salt formed, whilst from the latter only one of the former can be obtained for every two of the latter. ${ }^{1}$

Methods for the quantitative estimation of amygdalin have been proposed by Rieckher ${ }^{2}$ and Feldhaus. ${ }^{3}$ That of the latter is based upon the decomposition of the amygdalin by emulsin in aqueous solution and estimation of the hydrocyanic acid produced. Almonds are freed from oil, powdered and macerated with water for twenty-four hours. The hydrocyanic acid is then distilled off in a current of steam, received in ammoniacal water, and estimated as cyanide of silver. Anyone that has distilled bitter-almond water, or brought hydrocyanic acid into contact with powerful alkalies, will know that this method is very faulty. To obtain even approximate results I think it must be necessary that $(a)$ the flasks in which the maceration is conducted should be completely filled with the mixture, and $(b)$ the use of ammonia or other powerful base should be avoided.

In Rieckher's method the amygdalin is decomposed by hydrate of barium, a reaction which, according to Lehmann, takes place tolerably smoothly. One reason for preferring this process to Feldhaus's is, that the result can be checked by estimating on the one hand the ammonia liberated, and on the other the amygdalate of barium produced. The latter can be determined in the solution after expulsion of the ammonia by removing the excess of barium with carbonic acid gas, and then decomposing with sulphuric acid the amygrlalate of that metal which has been left in solution. From the sulphate of barium thus obtained the amount of amygdalin acted upon can be calculated. This method cannot, however, I think, be applied directly to almond meal deprived of fat, but only to the impure amygdalin obtained by exhausting with boiling alcohol and precipitating with ether.

Myronate of potassium crystallizes in rhombic prisms, which are freely soluble in water, sparingly in cold alcohol, but are dissolved by warm $\left(50^{\circ}\right.$ to $\left.60^{\circ}\right) 85$ per cent. spirit. Myronic acid itself is also soluble in cold strong spirit, but rapidly decomposes. In aqueous solution myronate of potassium is easily resolved by ferments, especially by the myrosin of white and black mustard (but not

${ }^{1}$ It is remarkable that Lehmann could find cane-sugar in the seeds of the apple, pear, cherry, plum, peach, and bitter almond, which contain crystallizable amygdalin, whilst sweet almonds yielded glucose only.

2 N. Jahrb. f. Pharm. xxiv. 65.

${ }^{3}$ Archiv d. Pharm. clxvi. 52. 
by emulsin) into volatile oil of mustard (sulphocyanide of allyl, $\S 29$ ), characterized by its extreme pungency, glucose and bisulphate of potassium. A quantitative estimation might possibly be made by freeing the finely-powdered seeds from oil, exhausting with warm 85 per cent. spirit, and digesting the tincture for some time with carbonate of barium. From the filtrate the alcohol might be recovered by distillation, the residue dissolved in water, and digested with myrosin at a temperature of about $40^{\circ}$, the barium being finally precipitated as sulphate by the addition of hydrochloric and sulphuric acid. One molecule of sulphate of barium indicates one of myronic acid. ${ }^{1}$

White mustard contains no myronic acid, but in its stead another glucoside, to which the name of sinalbin has been given. Like myronic acid, it dissolves in boiling 85 per cent. spirit, separating out again to a great extent on cooling. It is crystalline, insoluble in ether and bisulphide of carbon; sparingly soluble in cold, freely in hot alcohol, and in water. Alkalies colour it "yellow, nitric acid transiently blood-red. It reduces alkaline copper solution, and is precipitated by mercuric chloride and nitrate of silver. Warm solution of caustic soda converts it into sulphate and sulphocyanide of soda; myrosin into glucose, acid sulphate of sinapine and sulphocyanate of acrinyl $\left(\mathrm{C}_{7} \mathrm{H}_{7} \mathrm{O}, \mathrm{NCS}\right)$.

Sulphocyanate of sinapine, which also occurs in white mustard, is not glucosidal, and differs from sinalbin in being more easily soluble in cold alcohol, and in yielding the sulphocyanide reaction directly with ferric chloride. ${ }^{2}$

Ericolin and menyanthin ${ }^{3}$ are glucosides containing no nitrogen, but yielding easily volatile decomposition products. The former has been described in $\S 155$. Menyanthin is freely soluble in warm water and in alcohol, but insoluble in ether. Conc. sulphuric acid dissolves it with the gradual production of a reddishviolet colouration. Warming with dilute sulphuric acid resolves it into glucose and menyanthol, the latter possessing a penetrating: odour. Menyanthin is precipitated by tannic acid, but not by acetate of lead.

${ }^{1}$ Compare Will und Köörner, Annal. d. Chem. und Pharm. cxxv. 25\%, 1863. (Am. Journ. Pharm. xxr. 323.)

2 Compare Will und Laubenheimer, Annal. d. Chem. und Pharm. cxcix. 150, 1879 (Pharm. Journ. and Trans. [3], x. 918). Babo und Hirschbrunn, ibid. Ixxxiv. 10, 1852.

${ }^{8}$ Compare Kromayer, loc. cit.; Liebelt, Jahresb. f. Pharm. 119, 1877. 
Pinipicrin is described by Kawalier ${ }^{1}$ as being freely soluble in water and alcohol, insoluble in pure ether, and not precipitable by basic acetate of lead. Its decomposition-products resemble those of ericolin.

Of glucosides which yield either fixed or clifficultly volatile decomposition-products not possessing any characteristic odour, the following may be mentioned.

Coniferin is sparingly dissolved by cold, but freely by warm water and by alcohol. It crystallizes in glistening needles, and melts at $185^{\circ}$ (uncorr.). With conc. sulphuric acid it forms a violet solution, and when moistened with hydrochloric acid and phenol develops a blue colouration. Dilute acids resolve coniferin into sugar and a resinous substance; with emulsin it yields a crystalline decomposition-product. The latter, which is sparingly soluble in water and alcohol, can be removed by ether from its aqueous solution. Its melting-point lies between $73^{\circ}$ and $74^{\circ}$. Coniferin, when exposed to the air, gradually acquires a vanillalike odour, a change rapidly brought about by warming with dilute sulphuric acid and bichromate of potassium, and caused by the decomposition of the coniferin with the production first of the methyl-ethyl ether of protocatechuic aldehyde, and finally of ranillin $^{2}(\$ 155)$. The reaction with hydrochloric acid and phenol may be used in testing for coniferin microchemically in the cambium of conifers.

The identity of this substance with the coniferin of Tangel, ${ }^{3}$ detected in sections of conifers by the red colouration produced by conc. sulphuric acid and phenol, must be left for the present an open question. Müller ${ }^{4}$ has shown that the latter also occurs in most indigenous trees (Salix, Populus, Prunus, Acer, Quercus, etc.), and is to be met with in abundance, especially in the autumn, in the hard bast and alburnum. According to Müller, the phenol only hastens the appearance of the red colour.

Arbutin $^{5}$ is sparingly soluble in cold alcohol, ether, and cold

${ }^{1}$ Chem. Centralblatt, $705,724,1853$.

2 Compare Tiemann und Haarmann, Ber. d. d. chem. Ges. vii. 609, 1874 (Journ. Chem. Soc. xxvii. 895). See also Kubel, Journ. f. pract. Chem. xevii. $243,1866$.

3 Flora, lvii. No. $15,1874$.

${ }^{4}$ Ibid. No. 25.

"Compare Kawalier, Chern. Centralblatt, 761, 1852; Strecker, Annal. d. Chem. und Pharm. cvii. 288, cxviii. 292, 1861; Hlasiwetz and Habermann, ibid. clxxvii, 334, 1875 (Journ. Chem. Soc. xxix. 78, xxx. 298). 
water, but the latter solvent dissolves it with facility when warmed. Dilute sulphuric acid resolves it into glucose, hydroquinone and methyl-hydroquinone. Both of the latter can be extracted by shaking with ether, and, when warmed with dilute sulphuric acid and peroxide of manganese, yield quinone, recognisable by its characteristic iodine-like odour. Acetate of lead does not precipitate arbutin.

Daphnin ${ }^{1}$ differs from arbutin in being precipitated by acetate of lead. It is sparingly dissolved by cold, but freely by warm water and by alcohol, and is insoluble in ether. Alkalies colour it yellow. Acids and ferments resolve it into sugar and daphnetin; the latter can be partially sublimed without decomposition: Certain other constituents of mezereon bark yield umbelliferone $(\S 27)$ by dry distillation.

Salicin crystallizes in colourless needles and scales, which have a powerful action on polarized light, and are freely soluble in boiling water and alcohol, but much less so in cold. It is insoluble in ether. From aqueous solution it can be extracted by amylic alcohol (\$56). Hydrate of lead does not combine with it. Boiling with dilute acids decomposes salicin into sugar and saligenin or saliretin, both of which substances can be removed by shaking with ether. If an aqueous solution of salicin or saligenin is boiled with dilute sulphuric acid and bichromate of potassium, salicylous acid is produced. (Cf. $§ 25$.) Conc. sulphuric acid dissolves salicin, saligenin, and saliretin with production of a fine red colour. Salicin strikes a beautiful violet with Fröhde's reagent. These reactions can also be employed for the microchemical detection of salicin.

Populin yields benzoic acid in addition to the above-mentioned decomposition-products when acted upon by dilute acids (cf. \$ 26), and salicylous acid when warmed with chromic acid. With conc. sulphuric acid a red colouration is developed, and with Fröhde's reagent a violet, which, however, is somewhat less intense than that yielded by salicin under similar conditions. It is considerably less soluble than the latter in water and in alcohol, and can be removed from aqueous solution not only by amylic alcohol (like salicin), but also by chloroform and (with difficulty) by

${ }^{1}$ Compare Zwenger, Annal. d. Chem. und Pharm. xc. 63, 1858 (Amer. Journ. Pharm. xxxiii. 325). 
benzene $(55)$. Populin decomposes with far greater facility than salicin.

Ben:ohelicin, ${ }^{1}$ detected by Johanson in willow bark, forms colourless crystals, soluble in water and alcohol. With conc. sulphurie acid it turns yellow. Fröhde's reagent does not produce a violet colouration. Boiling with not over-dilute hydrochloric acid resolves benzohelicin into glucose, benzoic acid, and a resinous substance that dissolves blood-red in conc. sulphuric acid.

Philyrin ${ }^{2}$ is much less soluble in water and alcohol than is salicin, and yields under the influence of dilute acids, glucose and philygenin, a polymer of saligenin. Both philyrin and philygenin dissolve in conc. sulphuric acid with red colouration.

Phlorrhizin ${ }^{3}$ crystallizes in colourless needles sparingly soluble in cold water, easily in hot. Ethyl and methyl alcohol dissolve it with facility, ether with difficulty. Dilute acids resolve it into glucose and phlorrhetin. Conc. sulphuric acid forms red solutions with both phlorrhizin and phlorrhetin; with Fröhde's reagent a splendid royal-blue colouration is rapidly developed. Moistened with ammonia and exposed to the air, phlorrhizin turns yellow, reil, and finally blue.

Esculin can likewise be obtained in colourless needles soluble in 12.5 parts of boiling, 672 of cold water; in 24 of boiling, and 120 of cold absolute alcohol. Chloroform removes it from aqueous solution (\$55). Boiling with dilute acids resolves it into glucose and æsculetin. The latter yields a yellow solution with alkalies; it is also soluble in bisulphite of ammonia, and if such a solution be mixed with ammonia and shaken with air, at first blood-red and subsequently a deep blue colour is developed. The very characteristic blue fluorescence of rsculin is increased by alkalies and destroyed by acids.

Fraxin possesses a similar fluorescence, and can also be obtained in colourless crystals. It is more sparingly soluble in water and absolute alcohol than æsculin, but more freely in ether, to which it imparts its fluorescence. Ferric chloride is said at first to strike

${ }^{1}$ See Johanson, loc. cit. Piria, Annal. d. Chem. und Pharm. Ixxxi. 245, 1852 ; xcvi. 375, 1855 (Amer. Journ. Pharm. xxiv. 241, xxviii. 259).

2 Compare Campona, Annal. d. Chem. und Pharm. lxxxi. 245, xcvi. 375.

${ }^{3}$ For isophlorrhizin, see Rochleder, Journ. f. pract. Chem. civ. 397, 1868 (Amer. Journ. Pharm. xli. 419). 
a green colour and subsequently yield a yellow precipitate: Fraxin is thrown down by acetate of lead. ${ }^{1}$

Syringin (\$55) crystallizes in colourless needles, dissolves with difficulty in cold water, more easily in hot water and alcohol, but is insoluble in ether. Basic acetate of lead does not precipitate it. from aqueous solution. Conc. sulphuric acid dissolves it with deep blue colouration; Fröhde's reagent, blood-red passing to, violet; conc. nitric acid, deep red. Chloroform extracts syringin from aqueous solution. ${ }^{2}$

For globularin see Walz; for coriamytin compare $\S 155$; for pittosporin see v. Mü̈ller ; for samaderin see de Vrij. ${ }^{5}$

Colocynthin can be obtained in yellowish crystals which dissolve in conc. sulphuric acid with the gradual production of a fine red colour; Fröhde's reagent produces a cherry-red. It is extremely bitter, easily soluble in water and alcohol, but insoluble in ether: Benzene ( $\$ 55)$, or better, chloroform or amylic alcohol, extracts it from aqueous solution; it is precipitated by basic acetate of lead and by tannin.

Bryonin is also precipitated by the latter reagent. ${ }^{6}$

For ononin, which gradually assumes a cherry-red colour with conc. sulphuric acid, see Hlasiwetz. ${ }^{7}$

Apiin crystallizes in shining silky needles soluble in hot water or, more easily, in hot alcohol. Ether does not dissolve it. Ferrous sulphate colour's the aqueous solution blood-red. Both alcoholic and aqueous solutions gelatinize on cooling. In dilute alkalies apiin dissolves with yellow colouration.

For datiscin, which is also coloured yellow by alkalies, see Braconnot ${ }^{8}$ and Stenhouse. ${ }^{9}$ Ferric chloride precipitates it green ;

${ }^{1}$ For a number of other glucosides and allied substances (argyræscin, aphrodæscin, etc.) discovered by Rochleder in horse-chestnuts, see Journ. f. pract. Chem. Ixxxvii. 26, 1863 (Amer. Journ. Pharm. xxxv. 290).

${ }^{2}$ For the nearly allied ligustrin, see Kromayer, Archiv d. Pharm. cv. 9, 1861 ; for syringopicrin (easily soluble in water), ibid. cix. 26, 1862.

${ }^{3}$ N. Jahrb. f. Pharm. vii. 1, 1857 ; xiii. 281, 1860.

4 'The Organic Constituents of Plants,' Melbourne, 1878.

${ }^{5}$ Chem. Centralblatt, 92, 1859 ; Jahresb. f. Pharm. 208, 1872. See Blume, Amer. Journ. Pharm. xxxi. 342.

${ }^{6}$ N. Jahrb. f. Pharm. ix. 65, 217, 1859 (Amer. Journ. Pharm. xxxi. 249).

- Chem. Centralblatt, 449, 470, 1855.

8 Annales de Chimie et de Physique [2], iii. 277, 1816.

9 Annal. d. Chem. und Pharm. xcviii. 166, 1856 (Journ. Chem. Soc. ix. 226). For helianthic acid compare Ludwig und Kromayer, Archiv d. Pharm. [2], xcix. 1, 1848 (Amer. Journ. Pharm. xxxii. 135). 
accetute of lead, yellow. Zander ${ }^{1}$ has recently found a glucoside allice to datiscin in the sceds of Xanthium strumarium. For physalin, which can easily be extracted by shaking with chlorcform ( 555 ), see Dessaignes and Chautard ;2 for dulcamarin, see Gcissler. ${ }^{3}$ The latter is soluble in acetic ether, insoluble in ether, chloroform, bisulphide of carbon, and benzene. It is precipitated by tannin, and by basic acetate of lead. Conc. sulphuric acid dissolves it with red colouration passing to rose; with alkalies it forms reddish-brown solutions.

Hesperidin shows a disposition to form sphæro-crystals. It is sparingly soluble in water and cold alcohol, freely in warm alcohol and acetic acid, but insoluble in ether. Ferric chloride colours it brownish-red ; conc. sulphuric acid gradually bright red (limonin the same). Acetate of lead produces no precipitate. If i solution in dilute potash is evaporated to dryness, the residue is coloured red, passing to violet when warmed with excess of dilute sulphuric acid. Hesperidin can be recognised under the microscope as sphæro-crystals soluble in warm alcohol.

Crocin (polychroite) forms a dark red powder sparingly soluble in ether and water, more easily in alcohol. Conc. sulphuric acid colours it blue. Dilute acids resolve it into glucose and crocetin (insoluble in water), a saffron-like odour being developed during the decomposition. Basic acetate of lead precipitates crocin. ${ }^{5}$

Glycyrrhizin ${ }^{6}$ is deposited from glacial acetic acid in sphærocrystalline masses of prismatic needles. After purification with acetic acid it is almost insoluble in water (forming a jelly with it), lut may nevertheless be extracted (in combination with bases) from liquorice-root by that menstruum. It contains nitrogen, is sparingly soluble in absolute, more easily in boiling 90 per cent.

1 'Chem. iiber die Samen von Xanthium strumarium,' Diss. Dorpat, 1880.

N. Repert. f. Pharm. i. 216, 1851 (Amer. Journ. Pharm. xxv. 135, 136).

${ }^{3}$ Archiv d. Pharm. [3], vii. 289, 1875 (Pharm. Journ. and Trans. [3], vi. 1010).

${ }^{4}$ Compare Hoffmann, Ber. d. d. chem. Ges. ix. 250, 685, 1876 (Journ. Chem. Soc. xxx. 420,421); for aurantiin, murrayin, limonin, ibid.; also Hilger, ibid. 26 (Journ. Chem. Soc. xxix. 709). For naringin, see Archiv d. Pharm. [3], xiv. 139, 1879. See also Dehn, Zeitschr. f. anal. Chem. ii. 103, 1866 ; and Tiemann und Will, Ber. d. d. chem. Ges. xiv. 946, 1881.

5 See Weiss, Journ. f. pract. Chem. ci. 65,1868 ; Stoddart, Pharm. Journ. and Trans. [3], vii. $238,1876$.

${ }^{6}$ Compare Habermann, Annal. d. Chem. und Pharm. excvii. 105, 1879 (l'harm. Journ. and Trans. [3]. x. 45, 1879). Habermann changes the name to slycyrrhizic acid. 
alcohol, and is almost insoluble in ether. Conc. sulphuric acid precipitates it from aqueous solution; acetate of lead and chloride of calcium, from alcoholic. Neese's method for the quantitative determination of glycyrrhizin is based upon the precipitation by sulphuric acid.

For panaquillon, see Garriques $;^{1}$ for thevetin, compare de Vrij $;^{2}$ for chamcelirin, see Greene. ${ }^{3}$

Cyclamin (Primulin) is crystalline and dissolves freely in water; the solution froths when shaken. It is easily soluble in dilute alcohol also, but sparingly in absolute, and insoluble in ether. ${ }^{4}$ It is said to bear a close resemblance to saponin. (Cf. $\$ \$ 77$ et seq.; $\S 167$.

For gratiolin, see Marchand ${ }^{5}$ and Walz; ${ }^{6}$ for paridin, $\mathrm{Walz}^{7}$ and Delffs. ${ }^{8}$

For convallarin and convallamarin, see Walz. ${ }^{9}$ The former is sparingly soluble in water, but imparts to it the property of frothing; it is freely soluble in alcohol, but insoluble in ether. The latter dissolves more easily in water, is precipitated by tannin, and turns gradually violet when exposed to the air in contact with sulphuric acid. Warming with hydrochloric acid colours. it red. Convallamarin can be extracted by shaking with chloroform ( $\$ 55)$.

Helleborin and helleboreïn. ${ }^{10}$ - The former is sparingly soluble in cold water, freely in alcohol and chloroform; the latter easily in water, more sparingly in alcohol, and insoluble in ether. It can be extracted by shaking with chloroform (\$55). Both dissolve

1 Chem. Centralblatt, 721, 1854 (Amer. Journ. Pharm. xxvi. 511).

2 N. Jahrb. f. Pharm. xxxi. 1, 1869. Compare also Jahresb. f. Pharm. 112, 1877 ; Blas, Amer. Journ. Pharm. xli. 310.

3 Amer. Journ. Pharm. 1. 250, 1878.

4 Compare Mutschler, Annal. d. Chem. und Pharm. clxxxv. 214, 18 i (Year-book Pharm. 145, 1878). See also Luca, Compt. Rend. Ixxxvii. 297, 1878 (Pharm. Journ. and Trans. [3], vii. 876, 1877).

5 Journ. de Chim. méd. xxi. 517 (Amer. Journ. Pharm. xvii. 281).

${ }^{6}$ Jahrb. f. Pharm. x. 317, xiv. 70, 1852, xxi. 1, 1863, where certain other constituents of gratiola are also treated of. (Amer. Journ. Pharm. xxxi. 340).

7 Jahrb. f. Pharm. iv. 3, v. 284, vi. 10 ; N. Jahrb. f. Pharm. xiii. 174, 1860.

${ }^{8}$ Ibid. ix. 25, 1858.

9 Ibid. x. 145, 1858 (Amer. Journ. Pharm. xxi. 557).

${ }^{10}$ Compare Husemann and Marmé, Annal. d. Chem. und Pharm. cxxxv. 55 , 1865 (Pharm. Journ. and Trans. [2], vii. 621, 1866). 
in conc. sulphuric acil, with immediate production of a fine red colouration.

For digitalin and digitaleinn, see $§ 155$.

Scillain, a glucoside obtained from Scilla maritima, resembles digitalin in physiological action. It is sparingly soluble in cold water, but freely in alcohol, and when boiled with dilute hydrochloric acid is decomposed into sugar and a second substance, soluble in ether. Concentrated hydrochloric acid is coloured red when boiled with scillainn, and this is followed by the separation of a greenish flocculent deposit. Concentrated sulphuric acid dissolves it brown, with green fluorescence, and the solution is coloured bluish-red by bromine. Basic acetate of lead does not precipitate scillaïn. ${ }^{1}$

Saponin and digitonin are likewise glucosides; they have already been described in $\$ 77,78,79,155$, where mention has been made of their insolubility in absolute alcohol. My object in referring to them again here, is to draw attention to the resemhlance they bear, in many respects (frothing of the solution, etc.), to the preceding glucosides (cyclamin, etc.). The following substances are also allied to saponin :

Senegin-which, however, is possibly identical with saponinwas found by Christophsohn ${ }^{2}$ to differ from that body only in the rapidity with which the violet colouration was produced by sulphuric acid. It can be estimated by the methods detailed in $\$ i 8$.

Christophsohn also proved that both saponin and senegin are accompanied in the drugs yielding them by another substance that has a much more powerful action on the heart than either of those principles themselves. This other substance remains in solution after separation of the saponin by baryta-water, but could not be obtained in a state of purity.

Melanthin, found by Greenish ${ }^{3}$ in the seeds of Nigella sativa, is not precipitated by ether from alcoholic solution. It resembles saponin in being freely soluble in weak spirit, but may be distinguished by its slight solubility in water, and in the ease

${ }^{1}$ Compare Jarmerstedt, Archiv f. exp. Pathol. und Pharmacol. xi. 22, 1879 (Amer. Journ. Pharm. lii. 91).

2 Loc. cit.

${ }^{3}$ Sitzb. der Dorpater Naturf. Ges. 210, $1879 ; 91,1881$. Pharm. Journ. and Trans. [3], x. 909, 1013, xii. 681 . 
with which it splits up into sugar and melanthigenin when boiled with a dilute acid.

The so-called smilacin was also formerly regarded as allied to saponin, but the researches of Flückiger ${ }^{1}$ have shown that under this designation a mixture of substances has been described, the principal constituent of which was named parillin. This body stands in close relation to sapogenin, the decomposition-product of saponin; and as the latter is contained in sarsaparilla, ${ }^{2}$ it is probable that parillin is produced from it during the life of the plant. According to Flückiger, parillin is not soluble in cold water to any appreciable extent, but dissolves in 20 parts of boiling. It is taken up by spirit of sp. gr. 0.83 more easily than by stronger or weaker alcohol. ${ }^{3}$ Its reaction with conc. sulphuric acid resembles that of saponin. Boiled with 10 per cent. sulphuric acid it decomposes into sugar and parigenin, with production of a green fluorescence. A similar fluorescence is also observed when hydrochloric acid gas acts upon a solution in a mixture of chloroform and alcohol.

Sapogenin resembles parillin in most of its properties. Rochleder is of opinion that it still retains a little sugar, and is therefore really the result of an incomplete decomposition of saponin. The violet colouration gradually produced when sapogenin is dissolved in conc. sulphuric acid serves to distinguish the body from digitoresin, which, according to Schmiedeberg, yields a yellow solution. (See § 155.)

Indican may also be mentioned here, as, although it is not a substance that can be unconditionally ranked as a glucoside, it may nevertheless be compared with them as regards its constitution. By the decomposition of indican indigo-blue is produced, together with a kind of sugar called indiglucin. I leave it, however, an open question whether the formation of indigo-blue is preceded by that of indigo-white, which, it is true, readily yields that substance by absorption of oxygen. Indican appears to occur in many plants (leaves, etc.), but to undergo a partial decomposition when they are slowly dried, and the black or blue

${ }^{1}$ Compare Fliickiger and Hanbury, 'Pharmacographia,' 646.

2 Otten, 'Histiol. Unters. der Sarsaparillen,' Diss. Dorpat, 1876. Otten estimated the saponin by the methods given in $\S 78$.

${ }^{3}$ Archiv d. Pharm. [3], x. 535, 1877 (Pharm. Journ, and Trans. [3], viii. 488). 
colouration of the leaves produced thereby would arouse a suspicion of its presence. Cold spirit extracts indican; the solution is best evaporated in a current of dry air at the ordinary temperature. Foreign bodies may be removed from the aqueous solution by shaking it with freshly precipitated hydrate of copper, but the copper that simultaneously passes into solution must be subsequently removed by sulphuretted hydrogen. The aqueous solution must also be evaporated at the ordinary temperature, the residue dissolved in cold alcohol, and the indican precipitated by ether. Dilute acids decompose this unstable body, as above described, with production of indigo-blue.

The latter is characterized by its insolubility in water, alcohol, and ether and solubility in carbolic and fuming sulphuric acids. It can be sublimed, and yields indigo-white (soluble in water) when boiled with glucose and an alkali. Advantage might be taken of the latter property testing for indican in dried vegetable substances. The residue of the material after exhaustion with water might be boiled with an alkali and glucose; from the solution thus obtained the indigo-blue would be again precipitated by passing a current of air through it.

$\S 168$. The following bitter principles have not as yet been shown to be glucosides; but they are likewise sparingly soluble in ether, more freely in alcohol: Antiarin, ${ }^{1}$ aristolochia-bitter, ${ }^{2}$ calendulin ${ }^{3}$ (gelatinizes with water), californin ${ }^{4}$ (appears to be a mixture of alkaloids, of which loturin, which is strongly fluorescent in acid solution, is especially interesting); carapin, ${ }^{5}$ cratogin,${ }^{6}$ cusparin $^{7}$ (coloured green by nitric acid, red by mercurous nitrate); quinorins (quinovic acid, obtained by boiling quinovin or quinova-bitter with acids, is said to resemble cholic acid in gradually turning red with

${ }^{1}$ See de Vrij and Ludwig, Zeitschr. d. oesterr. Apoth. Ver. 92, 1868 (Amer. Journ. Pharm. xxxv. 474).

2 See Walz, Jahrb. f. Pharm. xxvi. 73. Gmelin's 'Organic Chemistry.'

${ }^{3}$ See Stoltze, Ber. Jahrb. f. Pharm. 1820.

${ }^{4}$ See Mettenheimer, N. Jahrb. f. Pharm. i. 341, 1870. Hesse, Ber. d. d. chem. Ges. xi. 1542, 1878 (Journ. Chem. Soc. xxxvi. 73).

${ }^{5}$ See Caventou, Vierteljahresschr. f. pract. Pharm. x. 422, 1861 (Amer. Journ. Pharm. xxxi. 231).

" See Leroy, Journ. de Chim. méd. xvii. 3.

7 See Saladin, ibid. ix. 388 (Amer. Journ. Pharm. v. 346).

8 Compare Gmelin, 'Handbook of Organic Chemistry.' Staeder's method of estimating quinovic acid in certain cinchona barks (N. Tijdschr. voor de Pharm. in Nederl. 152, 1878) was pronounced unsatisfactory by de Vrij (ibid. 306). 
sulphuric acid and sugar); cnicin $^{1}$ (is said to dissolve green in conc. hydrochloric, red in sulphuric acid. It can be extracted by shaking with benzene ( $\S 55)$, but is partially precipitated from aqueous solution by basic acetate of lead); geraniin, ${ }^{2}$ lactucin and its allies, ${ }^{3}$ linin, ${ }^{4}$ lupinin, ${ }^{5}$ mudarin, ${ }^{6}{ }^{\text {olivil },{ }^{7}}$ quercin ${ }^{8}$ (very slightly soluble in absolute alcohol); sparattospermin. ${ }^{9}$

$\S 169$. Aloins. - There is another group of non-glucosidal bitter principles to which I should like to direct attention ; viz., that of the aloins - a series of closely allied but not identical chemical individua. All the members of the group are soluble in water and alcohol, sparingly only in ether; but it must be observed that the separate aloins show notable differences in their behaviour to water. That obtained from Natal aloes is the most difficultly soluble, whilst the aloin of Cape aloes, which is possibly isomeric with nataloin, is comparatively freely dissolved. ${ }^{10}$

All the aloins can be obtained in yellow crystals, but show a great disposition to form supersaturated aqueous solutions in which, perhaps, they exist in an amorphous state and free from water of crystallization. From such solutions the aloin can be

${ }^{1}$ See Nativelle, Journ. de Chim. méd. xxi. 69, and Scribe, Comptes rendus, xv. 802. See also my article on the detection of foreign bitters in beer in the Archiv d..Pharm. [3], iv. 293, 1874; also Kubicki, 'Beitr. zur Ermittel. fremder Bitterstoffe im Biere,' Diss. Dorpat, 1874 (Pharm. Journ. and Trans. [3], v. 566, 1875), and Jundzill, 'Ueber die Ermittel. einiger Bitterstoffe im Biere,' Diss. Dorpat, 1873.

2 See Müller, Archiv d. Pharm. [1], xxii. 29, 1828.

${ }^{3}$ Compare Ludwig and Kromayer, Archiv d. Pharm. cxi. 1, 1862; also Kromayer, 'Bitterstoffe.'

${ }^{4}$ See Schroeder, N. Repert. f. Pharm. x. 11, 1861.

${ }^{5}$ Compare Landerer, ibid. i. 446, 1854. This lupinin must not be confounded with the glucoside of the same name discovered by Schulze and Barbieri in 1878. Compare Ber. d. d. chem. Ges. xi. 2200 (Journ. Chem. Soc. xxxvi. 467).

${ }^{6}$ Compare Duncan, Phil. Mag. x. 465.

7 Compare Pelletier, Annal. de Chim. et de Physique, iii. 105 ; and Sobrero, N. Jahrb. f. Pharm, iii. 286, 1855.

8 See Gerber, Archiv d. Pharm. xxxiv. 167, 1831.

${ }^{9}$ See Peckolt, Zeitschr. d. allgemeinen oester. Apoth. Ver. 133, 1878 (Pharm. Journ. and Trans. [3], ix. 162, 1878).

'10 According to Treumann's researches ('Beitr. z. Kenntniss der Aloë,' Diss. Dorpat, 1880) the following are the formulæ of the various aloins (containing water of crystallization) calculated to the same number of atoms of oxygen. Barbadoes aloin $=\mathrm{C}_{48} \mathrm{H}_{58} \mathrm{O}_{20}, 6 \mathrm{H}_{2} \mathrm{O}$; Cape aloin $=\mathrm{C}_{46} \mathrm{H}_{58} \mathrm{O}_{20}, 6 \mathrm{H}_{2} \mathrm{O}$; Socotra aloin $=\mathrm{C}_{45} \mathrm{H}_{52} \mathrm{O}_{20}, 6 \mathrm{H}_{2} \mathrm{O}$; Natal aloin $=\mathrm{C}_{45} \mathrm{H}_{56} \mathrm{O}_{20}$; Zanzibar aloin $=\mathrm{C}_{45} \mathrm{H}_{50} \mathrm{O}_{20}$ $6-7 \mathrm{H}_{2} \mathrm{O}$; Curaçao aloin $=\mathrm{C}_{44} \mathrm{H}_{50} \mathrm{O}_{20}, 6 \mathrm{H}_{2} \mathrm{O}$. See also Fliickiger, Schweiz. Wochenschr. f. Pharm. 331, 1870 ; Pharm. Journ. and Trans. [3], ii. 193, 1871. 
gradually obtained by diffusion, but it is often long before they deposit crystals (most easily obtained from Natal aloes). Ferric chloride colours them, without exception, greenish-black (Natal aloin very slowly); they are all gradually precipitated by basic acetate of lead; perchloride of platinum colours Barbadoes and Curaçao aloin by degrees red to violet, Socotra and Cape aloin greenish-brown, Natal aloin yellowish-brown; chloride of gold produces a moreor less fine raspberry-red, passing generallyinto violet; with strong hydrochloric acid, Natal aloin alone becomes violet; mercurous nitrate colours Barbadoes and Curaçao aloin reddish. All the aloins are precipitated from aqueous solution by brominewater, in the form of sparingly soluble brominated compounds, which contain frequently, but not invariably, 40 to 44 per cent. of bromine. The opinion expressed in my 'Chemische Werthbestimmung starkwirkender Droguen,' that these bromine-precipitates might be used in determining the value of the different varieties of aloes, was based upon some experiments of Kondracki's; ; but since Treumann has shown that one and the same aloin can yield more than one substitution-product, I have been shaken in this opinion. The applicability of another method of estimating the value of an aloes by ascertaining how much tannin is necessary to precipitate and redissolve one of the constituents, has also been rendered doubtful. I was convinced that this body precipitable by tannin was a decomposition-product of aloin, or possibly an amorphous modification, and that it acted directly as a purgative; Kondracki's experiments confirmed this supposition by showing that the more active an aloes was, the greater was the amount of tannin solution required in titrating. But as more recent experiments have proved that the aloins themselves when taken in sufficient quantity have a purgative action (whether direct or indirect, I am unable to say), and the attempts to compare the amount of aloin in an aloes with that of the substance precipitated by tannin have not met with success, I feel myself compelled to retract for the present the statements made in my 'Werthbestimmung' on this subject.

The aloin is accompanied in aloes by a resinous substance which does not dissolve when the aloes is treated with about 10 parts of water, but which is soluble in concentrated aqueous aloin-solutions, in hot water, and in alcohol. Another body, probably non-purgative, also occurs in dried aloe-juice; it is freely soluble in cold

${ }^{1}$ Beitr. z. Kenntniss der Aloe, Diss. Dorpat, 1874. 
water, and is possibly an oxyaloin. Bromine does not appear to precipitate it from aqueous solutions.

$\S 170$. Carthamin, etc.-Some substances, more freely soluble in alcohol than in ether, and characterized by their yellow colour, have been already mentioned in $\S 152$, in connection with quercitrin (rutin, robinin, luteolin, etc.), and whilst referring to them here, I will also allude to carthamin, the colouring matter of safflower. ${ }^{1}$ It has been obtained in the form of an amorphous powder, of an orange-green colour and metallic lustre. It is sparingly dissolved by water, but easily by aqueous alkalies and alcohol; from alkaline solution it is precipitated by acids. It dissolves in ether, and stains silk rose- or cherry-red.

\section{ALKALOIDS.}

$\$ 171$. Colour-reactions. - The following reagents may be recommended for producing colour-reactions with alkaloids: Pure sulphuric acid ; sulphuric acid, containing a little nitric acid (1 in 200 ) ; sulphuric acid, containing 0.01 gram of molybdate of soda in each cc. (Fröhde's reagent) ; sulphuric acid and sugar ; sulphuric acid and bichromate of potash; nitric acid (sp. gr. 1.3); conc. hydrochloric acid; ferric chloride. The reactions are best observed when a few drops of a solution (in alcohol, ether, chloroform, etc.) are allowed to evaporate in a small dish and a drop or two of the reagent added to the residue. In testing with sulphuric acid and sugar, it is generally better to mix the alkaloid as intimately as possible with 5 parts of sugar and add the sulphuric acid to the mixture. Delphinoidine should be mixed with as concentrated a solution of sugar as possible before the addition of sulphuric acid. If bichromate of potash and sulphuric acid are to be used in combination, it is advisable to dissolve the alkaloid in the acid and drop a crystal of bichromate into the solution. Sulphuric acid and nitrate of potash may be employed in the same way in place of the mixed sulphuric and nitric acids. Ferric chloride should be used in aqueous solution, and be as neutral as possible. ${ }^{2}$

Some of these reactions might be used in testing for alkaloids microchemically. The following table contains a few of the reactions of the more important alkaloids.

${ }^{1}$ Compare Schlieper, Annalen der Chemie und Pharm. Iviii. 357, 1846.

2 All these reactions are described at greater length in my 'Ermittel. d. Gifte.' 


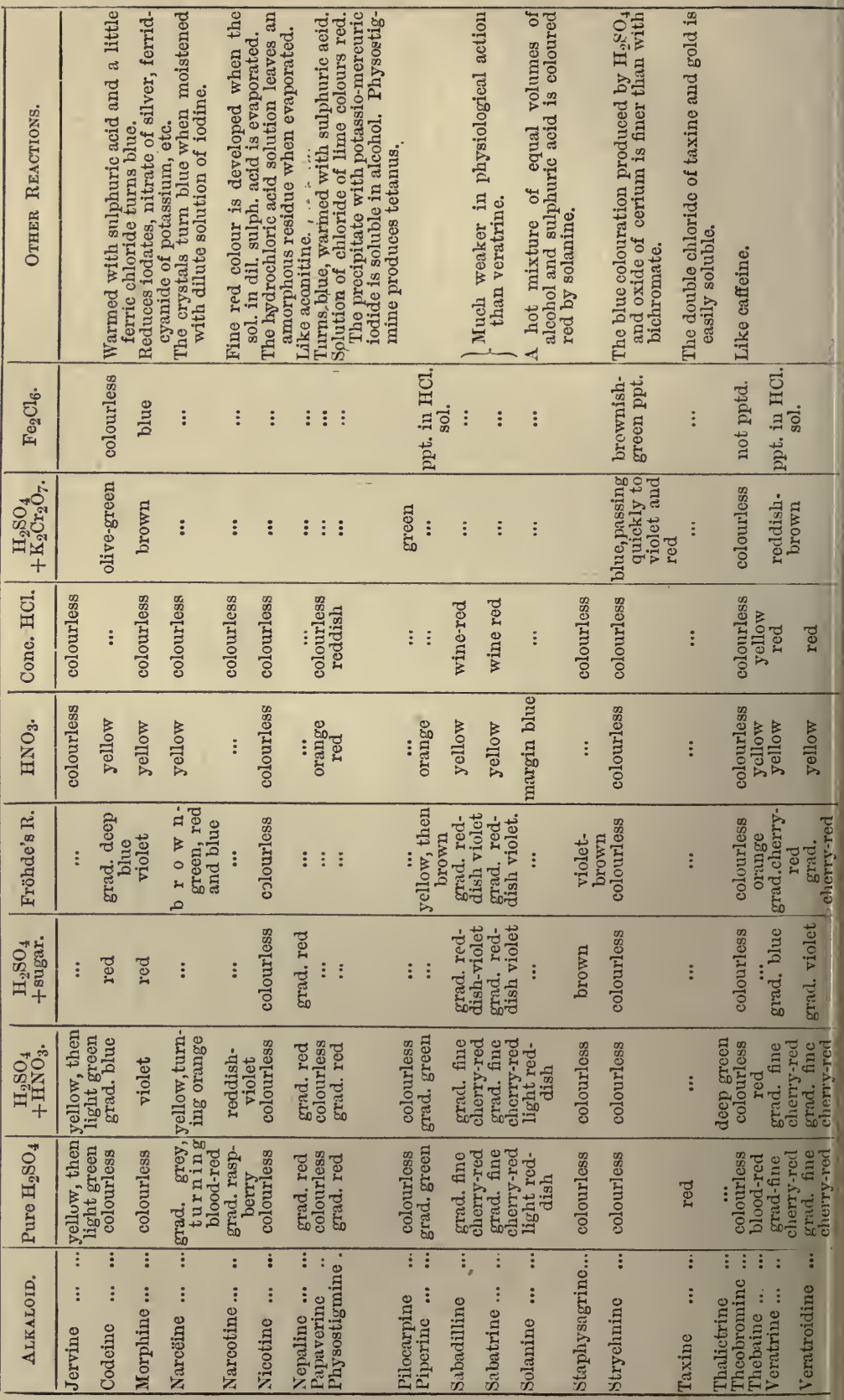


$\$$ 172. Other Tests.-For information concerning optical tests for alkaloids, see Buignet. ${ }^{1}$ Upon polarization (\$185), in particular, see Hesse. ${ }^{2}$ For the absorption spectra observable in colour-reactions of alkaloids, see Meyer ${ }^{3}$ and Poehl. ${ }^{4}$

The temperature at which alkaloids sublime has been determined by Armstrong, in an apparatus similar to that described in $\$ 17$ for ascertaining the melting-point of fats. The alkaloid is placed on a coverslip, to which a glass ring $\frac{1}{8}$ to $\frac{2}{3}$ inch high is cemented, and on which a second coverslip is laid. As soon as a cloud is observed on the latter, the temperature is noted. The sublimate is subsequently examined microscopically as to its crystalline or amorphous character. Mercury or easily fusible alloys may be used to heat the alkaloid. For the appearances observable during the microsublimation of alkaloids, see Helwig, Guy, Waddington, and others. ${ }^{5}$

The crystalline form of alkaloids has been closely investigated by Erhard. ${ }^{6}$

$\$$ 173. Platinum and Gold-salts.-The following list contains the percentage of platinum and gold in the double chlorides of those metals with some of the more important alkaloids (dried at $\left.100^{\circ}\right)$.

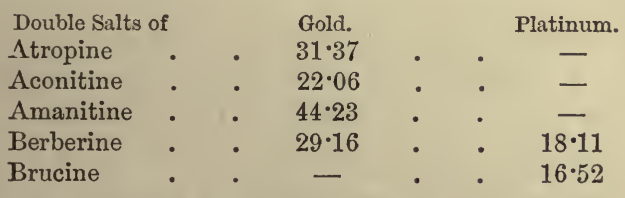

${ }^{1}$ Journ. de Pharm. et de Chim. [3], xl. 252, 1862 (Amer. Journ. Pharm. xxiv. 140).

2 Annal. d. Chem. und Pharm. clxxvi. 89, 1875 (Pharm. Journ. and Trans. [3], vii. 191), and cxcii. 161, 1878. See also Oudemans, ibid. clxxxii. 33, 1877 (Year-book Pharm. 75, 1878) ; Arch. Néerland. des Sciences exactes et naturelles, x. 193, 1875; and amongst older works that in particular of Bouchardat, Annales de Chimie et de Physique [3], ix. 213. See also Poehl's paper subsequently quoted.

${ }^{3}$ Archiv d. Pharm. [3], xiii. 413, 1878.

${ }^{4}$ Pharm. Zeitschr. f. Russland, 353, 1876.

${ }^{5}$ Compare Helwig, 'Das Mikroskop in der Toxicologie,' Mainz; Guy, Pharm. Journ. and Trans. [2], viii. 718 ; ix. 10, 58, etc. ; Waddington, ibid. [2], ix. 266, 409 ; Stoddart, ibid. 173 ; Brady, ibid. 234; Ellwood, ibid. [2], x. 152; Ledgewick, Brit. Rev. lxxxi. 262.

${ }^{6}$ N. Jahrb. f. Pharm. xxv. 129, etc.; xxvi. 9, etc., 1866. Amongst the older works are Hühnefeld's 'Chemie der Rechtspflege,' Berlin, 1823; Anderson, Chem. Centralblatt, 591, 1848; Taylor, 'On Poisons ;' Guy, 'Principles of Forensic Medicine;' Brand et Chaudé, 'Médecine légale,' Paris, 3858 . 


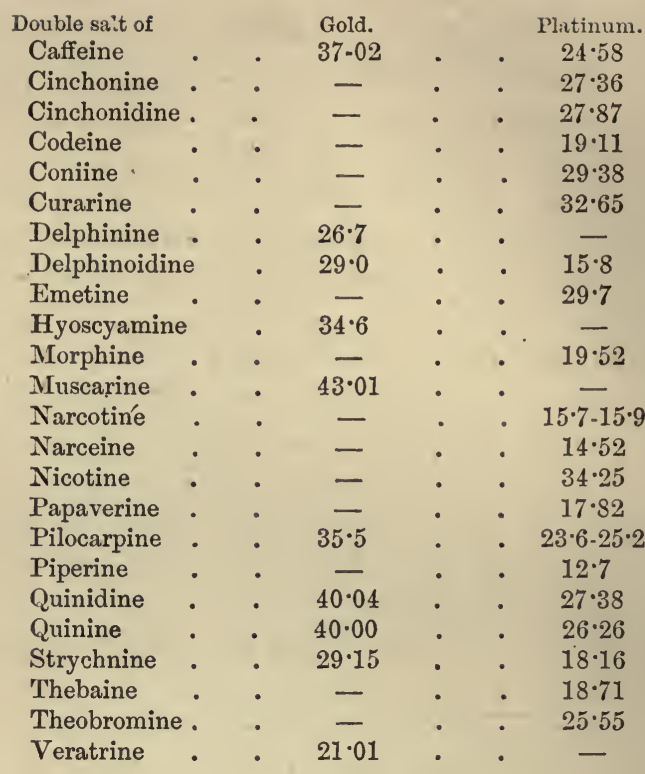

\$174. Estimation of Alkaloids.-In estimating the alkaloid in leaves or easily pulverizable stalks, it will frequently be found practicable to exhaust the powdered substance with spirit, evaporate the tincture, and extract the residue with acidulated water. The solution thus obtained may then be titrated with potassio-mercuric iodide, as directed in $\S 65$. But if the material contains much starch, or is difficult to powder (as, for instance, " aconite root), it is better to allow it to soak in about twice its weight of dilute sulphuric acid ( 1 in 30 ) before extracting with alcohol, as, otherwise, larger fragments of the substance are not uniformly penetrated by the spirit.

In estimating atropine, the drop-test (that is, the addition of a drop of the precipitating solution to a filtered drop of the liquid to be precipitated) cannot be used. It will be found advantageous to add at once sufficient of the reagent to precipitate the greater part of the alkaloid; after standing several hours, until the supernatant liquid has become clear, more of the reagent may be added, and so on as long as a precipitate is produced. The liquid clears more rapidly as the end is approached, till at last an interval of five to ten minutes is sufficient.

Atropine may also be estimated gravimetrically by adding 
excess of potassio-mercuric iodide, dissolving the precipitate in alcohol of 90 to 95 per cent., evaporating the filtered solution, and weighing the residue, which contains 40.9 per cent. of atropine.

For hyoscyamine the same precautions are necessary as for atropine. ${ }^{1}$

In estimating coniine gravimetrically with potassio-mercuric iodide, I obtained results that were far below the truth; the compound precipitated is somewhat freely soluble. (See also $\S \S 175,180$.

Nux Vomica and St. Ignatius' beans contain two alkaloids, strychnine and brucine, which differ in the intensity, at least, of their action on animals, and this fact must not be lost sight of in determining the value of those drugs by titration with potassiomercuric iodide. I have therefore proposed the following indirect method of determining both alkaloids : ${ }^{2}$

15 to 30 grams of the finely rasped seeds are exhausted by boiling three times in succession with dilute sulphuric acid ( 1 in 50), pressing the residue each time. The decoctions are united (about 700 cc.), nearly (but not quite) neutralizer with magnesia and evaporated to a syrup in the water-bath. To the residue 2.4 times its volume of 90 per cent. spirit is added, and after standing, the precipitated mucilage is filtered off and washed. The filtrate and washings are evaporated to about 30 to $50 \mathrm{cc}$. and, whilst still acid, well shaken with chloroform. The chloroform is then separated, the aqueous liquid made alkaline with ammonia, and the agitation with chloroform repeated as long as any alkaloid is removed. The alkaloidal residue obtained by evaporating the chloroformic solution is dried, weighed and dissolved in hydrochloric acid; the excess of acid is removed by evaporation, and the solution titrated with potassio-mercuric iodide. The weight of strychnine can be calculated from the expression $x=5.566(0.0197 \times c-m)$ and that of brucine from $y=6.566$ $(m-0.0107 \times c)$, where $c$ is the number of cc. of reagent used and $m$ the weight of the mixed alkaloids. It is still better to weigh the hydrochlorates of the alkaloids and calculate the strychnine salt from the expression $x=6.1733(0.02152 \times c-m)$, and the

I Compare my 'Werthbestimmung,' 32, and Thorey on the 'Distribution of Nitrogen in black and white henbane,' Diss. Dorpat, 1869, and Pharm. Zeitschr. f. Russland, 265, 333, 1865 (Pharm. Journ. and Trans. [3], xii. 874).

2 See my 'Werthbestimmung,' 64. Compare also Pharm. Zeitschr. f. Russland, 233, 1866. 
brucine salt from $y=7 \cdot 1733(m-0.01852 \times c), c$ being the same as in the previous expressions and $m$ denoting the weight of the mixed hydrochlorates.

Titration of morphine and narcotine with potassio-mercuric iodide serves only as a check on the weight of the alkaloids after isolation ( $\$ 182,187)$. The total alkaloid in opium cannot be estimated volumetrically with that reagent.

For a method of examining chelidonium compare $\$ 65$ and my 'Werthbestimmung,' p. 98 .

The presence in cevadilla seeds of three alkaloids, all of which act upon potassio-mercuric iodide, renders it impossible to do more with this reagent than compare the extracts of two or more different samples of seeds with one another. ${ }^{1}$ If an approximate separation of the three alkaloids is desired, it must be remembered that, according to the investigations of $\mathrm{W}$ eigelin, ${ }^{2}$ all three are removed together by shaking with chloroform; that sabadilline is almost insoluble in ether, but is dissolved by 150 parts of water at the ordinary temperature; that sabatrine is freely soluble in ether and soluble in 40 parts of cold water; and finally that veratrine is said to be taken up by 10 parts of ether and 1,000 of cold water.

The researches of Harnack and Witkowski have proved ${ }^{3}$ that the calabar bean also contains two alkaloids (calabarine and physostigmine), differing from one another in physiological action. For this reason the estimation of the total alkaloid by titration with potassio-mercuric iodide has only a limited value, but the alkaloids might possibly be separated, and estimated gravimetrically, as the calabarine precipitate is insoluble in alcohol whilst that produced by physostigmine is soluble.

$\S 175$. Coniine, pilocarpine, etc.-Zinoffsky has shown that coniine can be accurately estimated by phosphomolybdic acid in solutions free from ammoniacal salts. ${ }^{4}$ The strength of the reagent was such that 1 cc. precipitated 0.05 gram of coniine.

${ }^{1}$ Compare E. Masing, Archiv d. Pharm. [3], ix. 310, 1876 (Journ. Chem. Soc. xxxii. 367 ).

${ }^{2}$ Compare Weigelin, 'Unters. über die Alkaloïde der Sabadillsamen,' Diss. Dorpat, 1871 (Journ. Chem. Soc. xxv. 82S). See also P. G. A. Masing, 'Beitr. z. gerichtl. chem. Nachw. des Strychn ins u. Veratrins,' Diss. Dorpat, 1868.

${ }^{3}$ Compare Archiv f. exper. Patholog. und Pharmacol, v. 401, 1876 (Pharm. Journ. and Trans. [3], viii. 3).

4 'Die quant. Best. d. Emetins, Aconitins und Nicotins,' Diss. Dorpat, 1872 (Pharm. Journ. and Trans. [3], iv. 442). 
The same reagent has been employed by Poehl ${ }^{1}$ for the gravimetric estimation of pilocarpine, but that chemist admits that the results obtained are only approximate. By his method 10 grams of jaborandi leaves are extracted with $100 \mathrm{cc}$. of water containing 1 per cent. of hydrochloric acid; the infusion is precipitated with acetate of lead, the excess of which is removed by hydrochloric acid, and then, after filtration, phosphomolybdic acid is added. The precipitate is collected, washed with water containing a little hydrochloric acid, dried at $100^{\circ}$, and weighed. It is said to contain 45.66 per cent. of pilocarpine.

In estimating the alkaloid in solutions of the pure substance, phosphomolybdic acid would probably in many cases yield better results than potassio-mercuric iodide; but there is a certain danger attending its use, and that is the possibility in many cases of ammonia and amidic compounds being precipitated with and calculated as alkaloid. Of pilocarpine in particular it must be observed that, according to Christensen, the composition of the phosphomolybdic acid precipitate, as given by Poehl, requires revision.

Phosphomolybdate of quinine (dried below $70^{\circ}$ ) contains, according to Prescott, 27.3 per cent. of quinine.

For cases in which phosphotungstic acid may be employed see $\S 177$.

Attempts have also been made to estimate alkaloids by means of tannic acid, ${ }^{2}$ by either drying the precipitate produced or liberating the alkaloid from it with oxide of lead or other base, drying and weighing. My objection to the former of these two methods is that the tannates of the alkaloids are scarcely ever constant in their composition. The latter might be adopted in certain cases provided that the precipitated tannate is sufficiently sparingly soluble, and that the alkaloid itself is not attacked, as curarine is, by the oxide of lead used to decompose its tannate $(\S 64)$.

1 'Unters. d. Blätter des Pilocarpus officinalis,' St. Petersburg, 1877 (Yearbook of Pharm. 28, 141, 1881). See also Harnack and Meyer, Annal. d. Chem, und Pharm. cciv. 67, 1880 (Pharm. Journ. and Trans. [3], xi. 551, 587, 608) ; and Christensen, Pharm. Zeitschr. f. Russland, xx. 1881 (Pharm. Journ. and Trans. [3], xii. 400).

${ }^{2}$ Compare, for instance, Lefort, Journ. de Pharm. et de Chimie, ix. 117, 241, 1869 (Pharm. Journ. and Trans. [3], ii. 1029; iii. 63). See also my 'Werthbestimmung,' 40. 
Hager $^{1}$ and Hielbig ${ }^{2}$ have both experimented on the estimation of certain alkaloids by precipitation with picric acid. I have no doubt that in many cases very satisfactory results might be obtained by combining precipitation by picric acid with extraction by agitation with solvents, and in this opinion I have recently been confirmed by experiments published by Hager on the quantitative determination of nicotine. Hager recommends precipitation with picric acid at a temperature of $15^{\circ}$, washing with an aqueous solution of the precipitant, and finally drying at a temperature not exceeding $40^{\circ}$ to $50^{\circ}$. He found the nicotine precipitate to contain 27 per cent. of alkaloid.

$\$ 176$. Estimation of Caffeine.-I may supplement the method given in $\$ 66$ for the estimation of this alkaloid by the following remarks: Ether $^{3}$ extracts the alkaloid in a state of greater purity than chloroform, and yields therefore a correspondingly better result; but the mass must be very finely powdered, and the treatment with ether repeated several times to be certain of dissolving the whole of the caffeine. I have also used a mixture of 3 parts of ether with 1 of chloroform with success.

In estimating the alkaloid in guarana it is not advisable to extract with acidified water, nor is it necessary in determining the theine in tea.

Lieventhal ${ }^{4}$ extracted the powdered tea directly with chloroform, by which, however, far less than the total quantity of theine was dissolved. I must make the same objection to Claus's method, ${ }^{5}$ which consisted in extracting with ether, shaking the ethereal extract with dilute sulphuric acid, neutralizing the aqueous solution with magnesia, evaporating to dryness, and again extracting with ether. Moreover, it would be difficult to remove the whole of the theine from ethereal solution by shaking with acidified water. ${ }^{6}$

${ }^{1}$ Pharm. Centralblatt, x. 137, 145, 1871. Compare also Medin and Almèn, Jahresb. f. Pharm. 1871.

${ }^{2}$ Loc. cit.

${ }^{3}$ Compare Wurthner's investigations, Pharm. Zeitschr. f. Russland, 711, 1872 ; and Weyrich, 'Ein Beitr. z. Chemie des Thees und Kaffees,' Diss. Dorpat, 1872 (Journ. Chem. Soc. xxvi. 1235).

${ }^{4}$ Pharm. Zeitschr. f. Russland, 369, 1872 (Year-book of Pharm. 239, 1873).

${ }^{5}$ Pharm. Zeitschr. f. Russland, 357, 565, 1862.

${ }^{6}$ For the less recent methods of Péligot. and Zöllner, see my 'Chem. Werthbestimmung,' 59. For other methods see Comaille, Zeitschr. f. anal. 
\$ 177. Theobromine.-Trojanowsky found that the theobromine in cacao-seeds might be estimated by the following process $^{1}$ : 5 grams of the powdered seed are freed from fat by treatment with petroleum spirit, dried, rubbed down with powdered glass and water to a thin paste, mixed with an equal weight of calcined magnesia, and dried in the water-bath at $60^{\circ}$ to $70^{\circ} \mathrm{C}$. The residue is again finely powdered, and exhausted by boiling with 80 per cent. spirit. The decoctions are filtered whilst hot, and evaporated to dryness in a beaker. From the dry extract petroleum spirit will dissolve a little more fat; after having been again dried, the mass is thrown on to a tared filter, and washed with cold spirit till nearly colourless. It is then dried and weighed, and to the weight of theobromine thus obtained 0.0007 gram added for every cc. of wash-spirit. ${ }^{2}$

Wolfram estimates theobromine in cacao-seeds by precipitating with phosphotungstic acid ${ }^{3}(\S 64)$, and subsequently separating

Chem. xv. 474, 1876 (Year-book of Pharm. 20, 1876); Markownikoff, ibid. xvi. 127, 1877 (Year-book of Pharm. 104, 1877); Cazeneuve and Caillol, ibid. xvii. 221, 1878. The latter replace the magnesia in the above method with lime, and the ether with chloroform; Markownikoff also uses chloroform. In working upon coffee-beans it will be found very difficult to reduce them to the fine powder necessary to ensure the success of the estimation. This may be best accomplished after the beans have been thoroughly dried at $100^{\circ} \mathrm{C}$.; Weyrich, however, has shown that the amount of caffeine contained in a sample of coffee is no criterion of its quality, and even the estimation of the ash, potash and phosphoric acid in addition to that of the caffeine does not furnish data free from objection. Levesie estimated (Archiv d. Pharm. [3] viii. 298, 1876 ; Journ. Chem. Soc. xxxi. 752) fat, mucilage, tannin and cellulose, but with unsatisfactory results. The determinations of the theine, substances soluble in water, ash, etc., in tea, made by Weyrich, showed the possibility of detecting adulterations, but not of judging of the quality.

1 ' Beitr. zur pharmacog. und chem. Kenntniss des Cacaos,' Diss. Dorpat, 1875. This work also contains estimations of the other more important constituents of cacao (fat, ash, starch, etc.) in various samples.

2 According to determinations made at my request by Treumann, theobromine dissolves in 148.5 parts of water at $100^{\circ}$, and in 1,600 at $17^{\circ}$; in 422.5 parts of boiling absolute alcohol, and 4284 parts at $17^{\circ}$, and in 105 parts of boiling chloroform. It differs in its solubility from caffeine, with which, however, it shares the reaction with chlorine and ammonia. Shaking with benzene does not remuve theobromine from aqueous solutions (\$ 55). See Archiv d. Pharm. [3], xiii. 1, 1878 (Year-book of Pharm. 71, 1879). Basic acetate of lead does not precipitate theobromine from aqueous solution.

3 Zeitschr. f. anal. Chem. xviii. 346, 1879 (Year-book of Pharm. 48, 1879). He prepared his reagent by dissolving 100 grams of tungstate and 60 to 80 of phosphate of soda in $500 \mathrm{cc}$. of water acidulated with nitric acid. For the use of phosphotungstic acid as an alkaloid-reagent, see also Scheibler, Journ. f. pract. Chem. lxxx. 211, 1866. 
the alkaloid from the precipitate. Ten grams of the substance are rubbed down with water to a fine paste, then exhausted by boiling with the same menstruum, filtered and washed with boiling water (700 to $800 \mathrm{cc}$.), as long as alkaloid can be detected in the washings. The mixed aqueous solutions are precipitated with ammoniacal acetate of lead and filtered. The filtrate is made alkaline with caustic soda, evaporated to 50 cc., acidified with sulphuric acid, and again filtered. From the acid solution, which should contain about 6 per cent. of free sulphuric acid, the theobromine may be precipitated by warming with phosphomolybdic acid. The precipitate is collected when cool, washed with water acidified with sulphuric acid, and decomposed by warming with caustic baryta ; the excess of the latter is removed by sulphuric acid and the sulphuric acid by carbonate of barium. The mixture is then filtered whilst hot, evaporated to dryness, and weighed. By deducting the ash the amount of pure theobromine is found.

$\S 178$. Estimation of Piperine.-The following is Cazeneuve and Caillot's method: 10 grams of finely-ground pepper are mixed with 20 of slaked lime, and enough water to form a thin paste, boiled for fifteen minutes, and then evaporated to dryness on a water-bath ; the residue is finely powdered and exhausted with ether. The piperine obtained by evaporating the ethereal solution is recrystallized from alcohol and weighed. It would, I think, be better if the powdered pepper were first freed from fat by treatment with petroleum spirit. Possibly the alkaloid might then be purified by washing with petroleum spirit and water instead of recrystallizing from alcohol. (Compare also $\$ 64$.)

\$179. Acidimetric Estimation of Nicotine.-Schloessing's process, mentioned in $\S 68$, consists in exiracting the nicotine by passing the vapour of ether and ammonia through the tobacco, condensing the distillate, allowing the ether and ammonia to evaporate, and titrating the residual nicotine with dilute sulphuric acid. But the alkaloid retains ammonia, and the amount found is consequently too high. (Compare Kosutany and my "Werthbestim. mung.')

Wittstein, Brandl, and Liecke, all extract the tobacco with water acidified with sulphuric acid. Liecke evaporates to a syrup, precipitates with 2 volumes of alcohol, washes, and again evaporates. The residue is made alkaline with excess of potash,

${ }^{1}$ Zeitschr. f. anal. Chem. xvii. 379, 187 S (Year-book of Pharm. 42, 1878). 
and distilled (finally at a temperature of $260^{\circ}$ ) into a measured quantity of volumetric sulphuric acid, the excess of which is then determined by solution of soda. Wittstein and Brandl distil the acid extract direct with caustic potash, and note the amount of sulphuric acid necessary to neutralize the distillate. They then evaporate to dryness, extract with alcohol, which dissolves the sulphate of nicotine, and determine the sulphuric acid in the insoluble portion. This is then deducted from the quantity used, and from the difference the nicotine present is calculated.

Kosutany treats the leaves, previously soaked in water, with milk of lime until free from ammonia, then extracts with water, and shakes the filtered solution with petroleum spirit. From the latter, after separation, the alkaloid is removed by agitating with a known quantity of volumetric sulphuric acid, the excess of which is determined by baryta water. (Compare my "Werthbestimmung,' p. 55.)

$\S 180$. Coniine.-Similar methods have also been proposed for the estimation of coniine. I have already expressed my opinion of them in my 'Werthbestimmung,' p. 42, where I have at the same time pointed out that those processes which involve the evaporation of a solution of chloride of coniine, and determination of the alkaloid from the amount of chlorine in the residue, ${ }^{1}$ are open to objection on the ground that chloride of coniine is easily volatilized (\$§174, 65).

$\$ 181$. Separation of two Alkaloids.-Attention has already been drawn in $\S 69$ to cases of the occurrence of two alkaloids in vegetable substances. The remarks made in that section may be supplemented here with a few examples so taken as to include details of processes of more frequent applicability, and to give hints for the valuation of drugs in general use. Some such instances have already been described in $\S 174$, and I propose following these here with the discussion of a few more methods for the separation of only two alkaloids.

Let us first assume that the two alkaloids to be separated differ in their behaviour to acids, as, for instance, jervine and verctroüdine, alkaloids that occur in Veratrum album, lobelianum and viride. ${ }^{2}$

${ }^{1}$ Compare Hager, ' Untersuchungen,' ii. 250.

2 See Tobien, 'Beitr. z. Kenntniss der Veratrum Alkaloide,' Diss. Dorpat, 1877 (Pharm. Journ. and Trans. [3], viii. 80S) ; Bullock, Amer. Journ. Pharm. xlvii. 451, and xlix. 453 ; Wormley, ibid. xlviii. 4. 
Supposing, then, both these alkaloids to have been extracted together by shaking with chloroform, and to have been redissolved in dilute ( 2 per cent.) acetic acid, the addition of dilute sulphuric acid to such a solution would cause the precipitation of the majority of the jervine as sparingly soluble acid sulphate (Simon's vegetable baryta). According to Bullock this salt requires 427 parts of cold water for solution, and the precipitate contains 15.5 per cent. of sulphuric acid $\left(\mathrm{H}_{2} \mathrm{SO}_{4}\right)$. The hydrochloric acid precipitate $(6.55$ per cent. $\mathrm{HCl})$ is more soluble; the most advantageous method of all is to precipitate nitrate of jervine (soluble in 1,200 parts of cold water) by adding nitrate of potassium to acetic acid solution. Veratroidine remains in the filtrate, from which it can be extracted by shaking with chloroform. In a similar manner paricine may be separated from other cinchona alkaloids in the form of a sparingly soluble nitrate.

The behaviour of two alkaloids to bases will more frequently be found to present differences, of which advantage may be taken. Here two cases are to be distinguished : viz., either one alkaloid is precipitated, and the other left in solution; or, both are precipitated, but one is redissolved by an excess of the precipitant, whilst the other is not. As an instance of the first of these two cases, the separation of narcotine from narceine may be cited; the former is almost completely precipitated by ammonia, whereas the narceïne remains in solution. Morphine and codeine serve to exemplify the second case, which is of more frequent occurrence; excess of ammonia precipitates the former tolerably completely, but the codeine remains dissolved in the filtrate, from which it may be extracted by shaking with benzene. On the other hand, an excess of lime-water causes the separation of narcotine, but does not precipitate morphine. But nearly the whole of the latter alkaloid is thrown down if chloride of ammonium be added to the solution in caustic lime ( $\$ 187$ ).

This method of precipitation with excess of alkali is, however, unreliable in some cases, in which favourable results might have been anticipated. Strychnine can be separated from an acid solution very satisfactorily by the addition of excess of ammonia; brucine under the same conditions remains in solution until the greater part of the ammonia has volatilized. But if both alkaloids are present together, part of the brucine will separate with the strychnine on the addition of ammonia. ${ }^{1}$

1 See my 'Ermittelung von Giften,' 2nd edition, 259. 
Carbonated alkalies may occasionally be used instead of caustic. For the separation of some alkaloids bicarbonates have also been recommended, as it was found that under certain conditions one alkaloid would form a soluble carbonate, whilst the other was immediately precipitated in the free state. In this way paricine can be separated from the other bark alkaloids by bicarbonate of soda.

$\$ 182$. Behaviour to solvents. - Separation may be accomplished in this way, either by treating the dry alkaloids with the solvent, or by shaking it with the liquid containing the alkaloids in solution.

An instance of the first case may be found in the separation of brucine and strychnine from a mixture of the two alkaloids precipitated by ammonia. Absolute alcohol dissolves brucine with tolerable facility, but takes up only a minute proportion of strychnine. ${ }^{1}$ Another method, which I formerly employed for the separation of these two alkaloids, may also find a place here. It consisted in allowing the benzene solution of the mixed alkaloids to evaporate until the majority of the strychnine had separated, then quickly pouring off the mother liquor, washing with benzene, and evaporating to dryness ; the brucine was thus obtained, mixed with a little strychnine $(0.0683 \mathrm{gr}$. for every cc.). I have succeeded in separating the two alkaloids tolerably completely by both of these methods, but as they occasionally fail I cannot further recommend either of them; the first is, however, the more preferable of the two.

By treatment with water colchicine may be separated from the colchiceïne, which sometimes accompanies it in colchicum corms. The quantity of solvent used must not, however, be too small, as colchiceine is more soluble in concentrated aqueous solutions of colchicine than in pure water, which dissolves it but very sparingly. ${ }^{2}$

Moens, ${ }^{3}$ Stoeder, ${ }^{4}$ and Hielbig, ${ }^{5}$ found 40 per cent. spirit adapted for the separation of the cinchonine and 'amorphous

${ }^{3}$ Compare my 'Werthbestimmung,' 66. Even if the greater part of the free ammonia present be allowed to evaporate, the complete precipitation of brucine is a matter of difficulty ; that portion of the alkaloid that remains in solution must therefore be removed by shaking with benzene.

${ }^{2}$ See Hertel, Pharm. Zeitschr. f. Russland, Nos. 13 to 18, 1881 (Pharm. Journ. and Trans. [3], xii. 498).

${ }^{3}$ Nieuw Tijdschrift voor de Pharm. in Nederl., 322, $1869 ; 7,1870 ; 161$, 1875.

${ }^{\star}$ Archiv d. Pharm. [3], xiii. 243, 1878 (Journ. Chem. Soc. xxxvi. 281).

"Kritische Beurth. der Method. zur Trennung und quant. Best. d. Chinaalkaloide,' Diss. Durpat, 1880. 
alkaloid' of cinchona bark. The latter found 1 part of cinchonine dissolve in 1,100 parts of spirit of that strength, but, as the solution obtained in the separation of the alkaloids is not a saturated one, he recommends the addition of 0.0002 gram cinchonine for every cc. of such spirit used. He also found pure ether (free from water and alcohol) very suitable for the same purpose, as it dissolves so little cinchonine that a correction is scarcely necessary. The mixture of both alkaloids must be completely dried in the water-bath, and then very carefully powdered.

The separation of two alkaloids by means of ether may also be accomplished by allowing the ethereal solution of both to evaporate
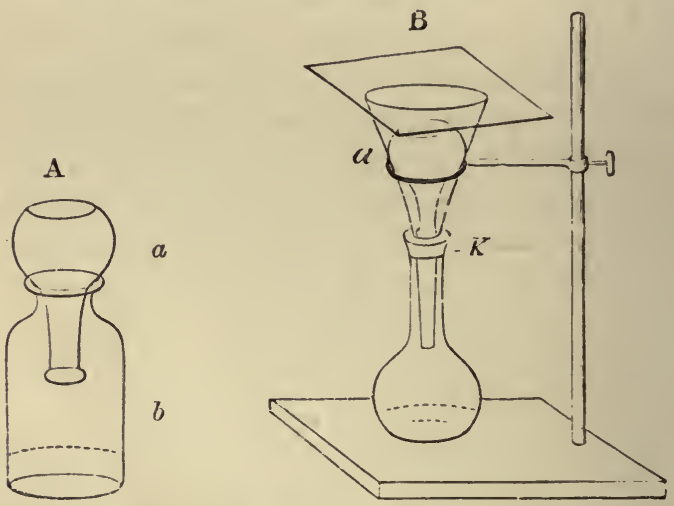

Fig. 8.

gradually, and, if one should separate in crystals, removing the other by slow washing with ether in the form of vapour. By this method I succeeded with Marquis ${ }^{1}$ in separating delphinine from delphinoïdine in perfectly colourless crystals.

The flask $a$, containing the mixed alkaloids (Fig. 8, A), was inverted in the wide-mouthed bottle, $b$, into which about $10 \mathrm{cc}$. of pure ether had been poured. The apparatus was then allowed to stand for several days at the ordinary temperature, during which ether-vapour from $b$ was continually condensing in $a$, and dropping back into $b$, saturated with delphinoïdine.

The apparatus figured in $8 \mathrm{~B}$, allows of the process being, to a certain extent, regulated; the funnel a, containing the flask, can be raised or lowered at pleasure.

${ }^{1}$ Archiv f. exper. Pathol. und Pharmacol. vii. 55, 1877. 
For the separation of morphine from narcotine by ether, see $\S 187$. Ether and chloroform (free from alcohol) can also be used to separate the former from codeine and thebaine. Morphine can be separated from thebaine, codeine, and narcotine, by the method of agitation; the last three are removed by benzene from ammoniacal solution, whilst scarcely traces of morphine are dissolved.

In a similar manner delphinine and delphinoïdine may be separated from staphisagrine by agitation with ether, in which the latter is insoluble ${ }^{1}$ after removing the first two, staphisagrine may be extracted with chloroform.

$\S 183$. Use of Salts, etc.; Separation of Quinine and Cinchonidine, etc. ${ }^{2}$-Instances of the use of salts in the separation of alkaloids may be found in the employment of tartrates for the quantitative estimation of quinine and cinchonidine in the presence of quinidine and cinchonine (cf. $\S 184, \mathrm{I}$.) ; by means of iodide of potassium or sodium, quinidine can be separated from cinchonine and 'amorphous alkaloid' (cf. $\S 184$, IV.). Wittstein recommended conversion into oxalates in alcoholic solution for separating strychnine and brucine; ${ }^{3}$ quinine may be freed from cinchonidine by precipitation as herapathite $^{4}$ (cf. $\S 184$, II.).

The separation of calabarine from physostigmine by potassiomercuric iodide, has already been described in $\S 174$; the same method might perhaps be feasible with chelidonine and sanguinarine. ${ }^{5}$

Chloride of gold can be used in separating muscarine from amanitine, as the double salt of the former is more soluble in water than that of the latter. ${ }^{6}$

Perchloride of platinum was the salt used in separating paytine from the other bark alkaloids, as the double salt of platinum with that alkaloid is very sparingly soluble in water. By means of the same salt, ammonia may be separated from those alkaloids and amides that yield double salts of greater solubility (§98). It must, however, be borne in mind that certain alkaloids undergo

1 See the paper by Marquis and myself previously quoted.

${ }^{2}$ Comp. Moens, loc. cit.; Johanson, Archiv d. Pharm. x. 418, 1877 ; Hielbig, loc. cit.

${ }^{3}$ Vierteljahresschrift f. pract. Pharm. viii. 409, 1859.

+ Compare Herapath, Pharm. Journ. and Trans. [1], xi. 448; xii. 6 ; de Vrij [3], vi. 461 ; N. Tijdschr. voor de Pharm. 1881; Hielbig, loc. cit.

5 See my 'Chem. Werthbestimmung,' 102.

${ }^{6}$ Compare Harnack, Archiv f. exper. Pathol. und Pharmacol. iv. 82, 1875. 
rapid decomposition when precipitated in combination with chloride of gold and platinum (e.g. curarine).

\$ 184. Quantitative Separation of Several Alkaloids from one another.-Such cases occur in the examination of cinchona barks. Although several of the many alkaloids in these barks are present in such minute quantities that they may generally be neglected, there are at least five the detection and estimation of which are of importance in valuing samples. These are quinine, cinchonidine, quinidine, cinchonine, and the so-called amorphous alkaloid. The mixed alkaloids are éxtracted and estimated as directed in $\S 67$. For their quantitative separation from one another, I propose using Moens' method, which has been recognised by Hielbig, after numerous experiments, as suitable for the purpose. ${ }^{1}$

I. The mixed alkaloids just referred to are dissolved in acetic acid, ${ }^{2}$ without the application of heat, and the solution evaporated to dryness, care being taken that the residue does not turn brown. This is then dissolved in the smallest possible quantity of water and filtered. From the solution, which should not be evaporated, quinine and cinchonidine are precipitated together by (about 5 of a gram of) tartrate of ammonia and soda, which is preferable to the Rochelle salt usually used. After standing twenty-four hours the precipitate is filtered off, washed, dried at $110^{\circ}$, and weighed. $1 \cdot 6$ gram of mixed alkaloids would yield about $30 \mathrm{cc}$. of filtrate, and require about the same quantity of wash-water. A correction must be made of 0.000746 gram of quinine and 0.000441 gram of cinchonidine for each cc. of filtrate and washings, provided that both alkaloids are present together. If the bark contains quinine alone, 0.00102 gram must be added for each cc. ; or if cinchonidine alone, 0.000543 gram. The apparent discrepancy in these figures is caused by the influence exercised by 1 the presence of either tartrate on the solubility of the other. 100 parts of precipitate indicate 79.41 anhydrous quinine or 76.8 cinchonidine.

II. To separate quinine from cinchonidine the mixed tartrates are dissolved in 90 to 92 per cent. spirit containing 1.6 per cent.

1 Loc. cit.

2 Hielbig has also experimented with hydrochloric and tartaric acids, but obtained the best results with acetic. The chlorides formed by the hydrochloric acid appear specially liable to cause errors when subsequently precipitating with tartrate. Whichever acid, however, be chosen, the excess must in some way be removed. 
of sulphuric acid; the filter used in the previous operation is also extracted with spirit of the same strength. One part of precipitate should yield about 20 parts of solution; from this the quinine is best precipitated by the reagent recommended by de Vrij, ${ }^{1}$ which is prepared as follows: To a solution of 2 parts of sulphate of quinoïdine in 8 of 5 per cent. aqueous sulphuric acid, a solution of 1 part of iodine and 2 of iodide of potassium in 100 of water is gradually added with constant stirring. The flocculent precipitate thus produced is slightly warmed till it agglomerates into a resinous mass, which is then washed with warm water, dried, and dissolved with application of heat in 6 parts of 92 to 94 per cent. spirit. After cooling the liquid is filtered off and evaporated to dryness, the residue redissolved in 5 parts of spirit, again filtered, and the filtrate used as the reagent. During the precipitation of the herapathite with this reagent, the liquid must be vigorously stirred to prevent the partial separation of cinchonidine in the form of orange flocks. If that has taken place the mixture must be warmed until the precipitate disappears. According to de Vrij, sufficient of the reagent has been added when an intense yellow colouration makes its appearance in place of a green precipitate of herapathite; the mixture is then heated to incipient ebullition, cooled, and its weight ascertained to allow of a correction for dissolved herapathite being subsequently made. Finally, the precipitate is collected on the filter previously used in separating the tartrates and washed with a saturated alcoholic solution of quinine-herapathite. After draining, the funnel is weighed with the filter, dried, and again weighed; the difference is the amount of herapathite solution retained by it, for each gram of which, as well as of mother liquor (not washings), a correction must be made of 0.00125 gram of quinine. 100 parts of herapathite dried at $100^{\circ}$ indicate 58.22 of anhydrous quinine. To ensure the success of the experiment, it is absolutely necessary that the herapathite should separate in the form of green glittering crystals, as otherwise the solubility differs from that here stated; amorphous herapathite, as well as some of the quinine compounds richer in iodine prepared by Jörgensen, are far more easily soluble. Unfortunately it sometimes happens, when working upon the mixed alkaloids separated from bark, that it is impossible to obtain the precipitate in this crystalline condition even

${ }_{1}^{1}$ Loc. cit. 
after three or four days. In this case a different correction must be made from that above mentioned, viz. 1 in 465 , as determined by Hielbig, instead of 1 in 600 . It is more advantageous to separate the quinine from the greater part of the cinchonidine by ether, then precipitate in the cold and filter off at once. ${ }^{1}$

III. The amount of quinine thus found is calculated into tartrate, and deducted from the weight of the mixed tartrates determined in I. ; from the difference the amount of cinchonidine present can be calculated.

IV. The filtrate and washings from the tartrate-precipitate are mixed with iodide of sodium (in the proportion of 0.5 gram for each gram of mixed alkaloids), evaporated to $20 \mathrm{cc}$. cooled, and then vigorously stirred. After standing twenty-four hours, the iodide of quinidine, etc., that has separated, is collected on a small tared filter, transferred to a small beaker, and triturated with $10 \mathrm{cc}$. of 95 per cent. spirit, returned to the same filter, and again treated with the same quantity of spirit. The residue is finally washed with $20 \mathrm{cc}$. of water, ${ }^{2}$ dried and weighed. 100 parts of precipitate correspond to 71.68 parts of anhydrous quinidine, to which a correction of 0.002481 gram has to be added for each cc. of filtrate and washings.

V. To the filtrate and washings from the last operation hydrochloric acid is added until perfectly clear, then considerable excess ( 2 to 3 grams) of carbonate of soda, and the mixture evaporated to dryness on the water-bath. The residue is reduced to the finest possible powder, transferred to a small dry flask, and extracted by maceration with pure ether, in successive portions of 10 to $20 \mathrm{cc}$. each, as long as any colour is removed. The ethereal filtrates are evaporated, the residue dried and weighed as amorphous alkaloid after deducting the quinine that has escaped precipitation as tartrate.

VI. The portion insoluble in ether is freed from that liquid by warming, and treated with water to remove soda, etc.; the cinchonine is then filtered off, washed, dried at $110^{\circ}$, and weighed. Traces of that alkaloid adhering to the filter used in filtering the ethereal solution and to the sides of the flask, may be dissolved

${ }^{1}$ Compare Christensen, Pharm. Zeitschr. f. Russland, 1881 (Pharm. Journ. and Trans. [3], xii. 441; de Vrij, ibid. 601).

2 The object of washing with spirit is to redissolve any iodide of cinchonine or amorphous alkaloid that may have separated out. It is important that the relative proportions of liquid, wash-spirit, and wash-water should be observed. 
in hydrochloric acid, added to the aqueous filtrate containing soda, etc., and removed from solution by shaking with chloroform. The alkaloid thus isolated must be weighed and added to the amount previously found, from which sum, however, the cinchonidine and quinidine left in solution must be deducted if great accuracy is required.

Hielbig also describes a second process for determining quinidine, cinchonine, and amorphous alkaloid, as follows :

VII. The filtrate and washings from the precipitated tartrates are evaporated to $20 \mathrm{cc}$, and for each gram of mixed alkaloids 0.5 gram of iodide of sodium, dissolved in $5 \mathrm{cc}$. of water and 15 of 90 per cent. spirit, is added, and the whole allowed to stand for twenty-four hours in a cool place. The iodide of quinidine is then collected on a tared filter, washed with a little water, dried at $100^{\circ}$, and weighed. (No correction is necessary for the alkaloid left in solution.)

VIII. The filtrate from the last operation is treated as in V., but the precipitate produced by the soda solution is here filtered off and the alkaloid still retained by the liquid extracted by shaking with chloroform. Both portions are then transferred to a beaker, and macerated with 40 per cent. spirit to remove amorphous alkaloid. It is best to cool the mixture and agitate, repeating the treatment as long as the spirit becomes coloured. The cinchonine is finally filtered off, dried, and weighed, 0.000202 gram being added for each cc. of spirit used.

IX. The alcoholic solutions are evaporated to dryness at $110^{\circ}$, and from the weight of the residue the quinine, cinchonidine, and cinchonine, previously reckoned as 'correction,' deducted. The remainder is to be regarded as amorphous alkaloid.

If the bark contain so little quinine and cinchonidine that after the addition of tartrate only single crystals are deposited on the sides of the beaker where touched by the glass rod, in quantity too small to allow of their being weighed, it may be assumed that the liquid contains at least the amount of alkaloid equal to the correction to be made. The actual presence of quinine may be detected by the thalleioquin reaction $(\$ 171)$ : if that yield a positive result, the presence of cinchonidine must remain a matter for conjecture; but if the result be negative, the precipitate may be assumed to consist of cinchonidine, and its quantity calculated from the correction to be made. 
The same course may be pursued when only traces of quinidine are precipitated.

§ 185. Behaviour to Polarized Light.-Attempts have also been made in examining barks to take advantage of the differences the alkaloids show in their behaviour towards polarized light, but the requisite accuracy does not seem to have been yet attained. ${ }^{1}$ In working with mixtures of the pure alkaloids, the results are, it is true, very satisfactory; but as soon as the mixed alkaloids separated from barks are examined the errors increase, as even small quantities of contaminating impurity can exercise a considerable influence on their action on a ray of light.

The most feasible is Oudemans' method of estimating quinine and cinchonidine. The alkaloids are precipitated as tartrates, and redissolved in hydrochloric acid (to 0.4 gram precipitate about $3 \mathrm{cc}$ normal acid, and water to $20 \mathrm{cc}$ ). Such a solution of quinine shows a rotation $[\alpha]_{\mathrm{D}}=-215 \cdot 8^{\circ}$; of cinchonidine $[\alpha]_{\mathrm{D}}=-131 \cdot 3^{\circ}$. The calculations may therefore be made according to the formula :

$$
215 \cdot 8 x+131 \cdot 3(100-x)=100(a)^{m}
$$

where $x$ is the percentage of tartrate of quinine, and $(a)^{m}$ the specific rotatory power of the mixture.

$\S 186$. Other Cinchona Alkaloids.-The following are some of the cinchona alkaloids of less frequent occurrence :

Aricine, ${ }^{2}$ the sulphate of which swells up to a jelly in chloroform.

Cusconine ${ }^{3}$ the neutral sulphate of which gelatinizes in aqueous solution, and does not dissolve in more sulphuric acid. Acetate of cusconine is also gelatinous.

Quinamine. This alkaloid occurs notablyin Cinchona succirubra, and generally remains associated with the 'amorphous alkaloid'

${ }^{1}$ Compare the papers quoted in $\S 172$ by Oudemans, Hesse and Hielbig. For the application of fluorescence, see Kerner, Zeitschr. f. anal. Chemie, ix. 135,1870 .

2 Compare Hesse, Annal. d. Chem. und Pharm. clxxxi. 58, 1876 (Pharm. Journ. and Trans. [3], vii. 331, 1876).

${ }^{3}$ See Hesse, Ber. d. d. chem. Ges. ix. 742, 1876 (Year-book Pharm. 226, 1880).

${ }^{4}$ Compare Hesse, Ber. d. d. chem. Ges. x. 2152, 1877 (Year-book Pharm. 62, 1878) ; Annal. d. Chem. und Pharm. cxcix. 333, 1880 (Year-book Pharm. 34, 1880); de Vrij, N. Tijdschr. voor de Pharm. en Nederl. 69, 1877. Oudemans, Annal. d. Chem. und Pharm. excviii. 135, 1879 (Year-book Pharm. 57, 1879; 34, 1880). 
isolated in the examination of the bark. It may be separated as follows: The mixed alkaloids are dissolved in dilute acetic acid, and to the solution sulphocyanide of potassium is added until the colour is only pale yellow. After standing till perfectly clear it is filtered, the filtrate made alkaline with ammonia and shaken with ether. The residue obtained by evaporating the ethereal solution is then recrystallized from alcohol. Quinamine dissolves in 32 parts of ether, and is also soluble in boiling petroleum spirit. The precipitate produced with chloride of gold rapidly decomposes with production of a red colouration.

For paricine, see $\S 181$; paytine, $\S \S 183,189$. Hesse states of the latter that it is coloured purplish-red by chloride of gold, and red passing to blue by chlorinated lime.

For other cinchona alkaloids, see Hesse in the papers, etc., already quoted. ${ }^{1}$

$\S 187$. Estimation of Opium.-Many methods have already been proposed for the estimation of the more important opium alkaloids. I have criticized them at length in my 'Chemische Werthbestimmung,' and restrict myself, therefore, here to recapitulating the modification of the Guibourt-Schacht's process there recommended, adding a few remarks on methods that have appeared since the publication of that work.

I. Five to ten grams of powdered opium are triturated with water to a very thin paste, macerated twenty-four hours and filtered. The residue is again treated in the same manner, and finally washed on the filter until the washings are colourless. When dried the insoluble portion should not amount to more than 40 per cent. of the opium employed. It still contains narcotine, which may be estimated according to VI.

II. The aqueous infusions and washings are evaporated in the water-bath until reduced to about five times the weight of the opium employed, cooled, filtered if necessary, and mixed with the slightest possible excess of ammonia. ${ }^{2}$ It is then vigorously stirred,

${ }^{1}$ Compare also Ber. d. d. chem. Ges. xi. 1938, 1878 (Pharm. Journ. and Trans. [3], xi. 839, 1881); Annal. d. Chem. und Pharm. ccv. 194, 211, 1880 (Year-book Pharm. 24, 27, 28, 1879; 42-44, 1881).

${ }^{2}$ See Cleaver, Amer. Journ. Pharm. xlviii. 359, 1876 (Pharm. Journ. and Trans. [3], vii. 240), and my remarks in the Jahresb. f. Pharm. 175, 1876. Cleaver, who also employs a modification of Mohr's process, recommends the opium to be previously exhausted with bisulphide of carbon, which removes substances that interfere with the subsequent operations. 
and allowed to stand exposed to the air, with occasional agitation, until the excess of ammonia has disappeared (not longer). The precipitated mixture of morphine, narcotine, and meconate of calcium is filtered off and dried. It should amount to not less than 14 per cent. of the opium used. Filtrate and washings are treated according to $\mathrm{V}$.

III. The precipitate is removed from the filter, reduced to the finest possible powder, and macerated with pure ether in a dry flask as long as narcotine is removed. The ethereal solutions are filtered through the same filter, evaporated to dryness at $110^{\circ}$, and weighed, or instead of weighing the residue may be dissolved in water acidulated with sulphuric acid, and titrated with potassiomercuric iodide $(\$ 65)$. The weight is noted as the amount of narcotine soluble in water.

IV. The residue insoluble in ether is dried and exhausted with boiling alcohol of specific gravity 0.81 , which removes morphine, and leaves meconate of calcium undissolved. The alcoholic solutions are filtered through the filter already used in the extraction with ether. The weight of the alkaloid can be ascertained either by evaporating to dryness, redissolving in acidulated water, precipitating with ammonia, and weighing, or by evaporating, redissolving in dilute sulphuric acid, and titrating according to $\S 65$. Good opium contains at least 8 per cent. of morphine.

V. If the morphine is reprecipitated for gravimetric estimation the filtrate may be mixed with the filtrate from II., made alkaline with ammonia, and shaken with amylic alcohol. All the morphine in solution is thus removed, and the amount which escapes precipitation in II. is sometimes very considerable. The amylic-alcohol solutions are evaporated to dryness, the residue dissolved in a little acidulated water, precipitated with a slight excess of ammonia, dried, weighed, and noted as morphine. A correction of 0.001 gram for each cc. of mother-liquid may be made if desirable.

VI. If the sample under examination is an opium of good quality the insoluble residue from I. will contain narcotine, but no morphine. The former may be estimated by extracting with water acidulated with sulphuric acid, precipitating with ammonia, filtering, washing, redissolving in dilute sulphuric acid, and titrating with potassio-mercuric iodide ( $\$ 65)$.

$\S 188$. Other Methods. - Weak spirit was also formerly employed 
in the place of water for exhausting the opium, and Proctor ${ }^{1}$ has recently proposed triturating the opium (13 grams) with water (15.5 gram) to a paste in a warm mortar, adding methylated spirit (46 grams) by degrees, and exhausting by percolation with the latter menstruum. The solution is evaporated to a syrup, mixed with water (63 grams), and filtered. The filtrate is again evaporated (to $6 \mathrm{cc}$.), mixed with an equal volume of methylated spirit and slight excess of ammonia, allowed to stand twelve to eighteen hours, filtered and the precipitate washed, first with a mixture of equal quantities of methylated spirit and water, and finally with the latter alone (about 31 grams). Proctor removes narcotine with benzene.

For the approximate estimation of the morphine Prollius recommends extracting the opium with 10 parts of 34 per cent. spirit, mixing the solution with 5 parts of ether and 0.2 of ammonia, allowing to stand twelve to twenty-four hours, filtering off, drying and weighing the morphine, which separates at the line of demarcation; narcotine is said to be dissolved by the ether.

Flückiger ${ }^{2}$ exhausts 8 grams of powdered opium by agitation for twelve hours with 80 grams of water, and filters the infusion through a filter $125 \mathrm{~mm}$. in diameter. 42.5 grams of the filtrate are mixed with 12 grams of alcohol of sp. gr. 0.812, 10 grams of ether, and 1.5 caustic ammonia in a tared flask, and set aside for a day or two. The crystals of morphine that have separated are then collected on a double filter 4 inches in diameter; both flask and residue on the filter are washed, first with a mixture of 6 grams of spirit with 5 of ether, and afterwards with 10 grams of ether. The crystals are finally gently pressed, returned to the flask, dried, and weighed. To the amount thus obtained Flückiger adds $0 \cdot 1$ gram (Mylius 0.088) for loss in precipitating and washing.

For the rotatory power of opium alkaloids, see Hesse. ${ }^{3}$

$\S 189$. Other Alkaloids.-In treating of the more important

${ }^{1}$ Pharm. Journ. and Trans. [3], vii. 244, 1876 ; viii. 211, 1877.

2 Pharm. Zeitung, Nos. 57, 59, 1879 (Pharm. Journ. and Trans. [3], x. 254, 1879). See also Van der Burg, Pharm. Weekbl. No. 26, 1879; Mylius, Archiv d. Pharm. [3], xv. 310, 1879 (Year-book Pharm. 22, 23, 1880).

${ }^{3}$ Annal. d. Chem. und Pharm. clxxvi. 189, 1875. See also Yvon, Journ. de Pharm. et de Chim. xxix. 372, 445, 1879. A paper on the rarer opium alkaloids was published by Hesse in the Annal. d. Chem. und Pharm. cliii. 47, 1870 (Pharm. Journ. and Trans. [3], i. 205, 1870). 
alkaloids, I have described such of their properties only as are of importance for the object of this work, and refer students that may desire more minute details to any good text- or hand-book of chemistry. I may be permitted to give a few literary references, and make a few observations on some of the alkaloids with which we are less familiar, and about which little or no information is to be found in text- or hand-books, in case it should be necessary in the course of an analysis to compare a substance with any one of them.

For ergotinine and picrosclerotine, compare Tanret ${ }^{1}$ and Blumberg. ${ }^{2}$ Two volumes of conc. sulphuric acid colour an aqueous solution of the former, first red, then bluish-violet, of the latter violet. With an equal volume of Fröhde's reagent, both are coloured violet, passing to blue. Both can be extracted from solution by agitation with ether. The latter, which is resinous and very sparingly soluble in water, is possibly a decompositionproduct of the former.

For curarine, which is freely soluble in water, see Preyer ${ }^{3}$ and Sachs. ${ }^{4}$ This alkaloid cannot be removed from solution by shaking with ether, etc. Its reactions are described in $\S 1.71$. Chloroform extracts small quantities (sufficient for the reactions and physiological experiments) of the alkaloid from the resiaue (finely powdered) obtained by evaporating its aqueous solution. (Compare also $\S ~ 64,68,182$.)

For erythrophloeine see Gallois and Hardy. ${ }^{5}$ It is soluble in water, can be extracted by shaking with acetic ether, and is coloured violet by sulphuric acid and permanganate of potassium.

Lobeliine, see Lewis and Richardson. ${ }^{6}$ (Cf. §56.)

1 Repert. de Pharm. N. Sér. iii. 308, 1875 ; v. 226, 1877 (Pharm. Journ. and Trans. [3], vii. 249.1876 ; vi. 522, 1875).

2 Ein. Beitr. z. Kenntniss d. Mutterkornalk. Diss. Dorpat, 1878 (Pharm. Journ. and Trans. [3], ix, 23).

3 Zeitschr. f. Chem. vi. 382, Compt. rend. 1. 1828, 1865. See also Koch, 'Vers. über die Nachweisbarkeit d. Curarins in thier. Flüssigk. und Geweben,' Diss. Dorpat, 1870, and my 'Beitr. z. gerichtl. Chem.' 170, St. Petersburg, 1871.

4 Annal. d. Chem. u. Pharm., cxci. 254, 1878 (Journ. Chem. Soc. xxxiv. 517). See also my observations in the Jahresb. f. Pharm. for the same year.

5 Union Pharm. xvii. 202, 1876 (Pharm. Journ. and Trans. [3], vii. 77, 1876), also xix. $359,1878$.

${ }^{6}$ Amer. Journ. Pharm. 293, 1872 ; Pharm. Journ. and Trans. [3], viii. 561, 1878. See also my 'Beitr. z. gerichtl. Chem.' 18. 
Conessine or wrightine, see Haines ${ }^{1}$ and Stenhouse. ${ }^{2}$ It is very sparingly soluble in alcohol, ether, and bisulphide of carbon.

Harmaline and harmine, compare Fritšche. ${ }^{3}$ The former yields yellow salts with acids-the latter colourless. Both are somewhat sparingly soluble in alcohol.

Surinamine is also sparingly soluble in spirit. (Compare Hüttenschmidt and Winkler. ${ }^{4}$ )

Aribine, see Rieth. ${ }^{5}$ It is sparingly soluble in ether, as is also Atherospermine, compare Zeyer ${ }^{6}$ and Rhoeadine, compare Hesse. ${ }^{7}$ The latter is colourless, but is converted by dilute acids into deep red rhœagenine.

Violine, compare Boullay ; ${ }^{8}$ for beberine, see Maclagan ${ }^{9}$ (cf. § 171); for belladonnine, see Hübschman ;10 for cocaïne and hygrine, compare Niemann, Wöhler, and Lossen. ${ }^{11}$ Concentrated hydrochloric acid decomposes cocaïne into benzoic acid, and the alkaloidal ecgonine.

For chlorogenine and porphyrine see Hesse. ${ }^{12}$ Chlorogenine in acid solution shows a powerful blue fluorescence.

Corydaline, compare Wackenroder, Müller and Leube, Boedecker and Wicke. ${ }^{13}$ The alkaloid dissolves in conc. sulphuric acid, with dark red colouration.

Cytisine, compare Husemann and Marmé. ${ }^{14}$

1 Pharm. Journ. and Trans. [2], vi. 432.

${ }^{2}$ Ibid. [2], v. 493; Schweizerische Wochenschrift f. Pharm. 172, 174, 1865.

${ }^{3}$ Chem. Centralblatt, $1847-49,1853,1854$. See also Goebel, Annal. d. Chem. und Pharm. xxxviii. 363, 1841.

4 Gmelin's 'Handbook of Chemistry.'

${ }^{5}$ Chem. Centralblatt, 903, 1861 (Amer. Journ. Pharm. xxxiv. 395).

6 Vierteljahresschr. f. pract. Pharm. x. 513. 1861 (Amer. Journ. Pharm. xxxiv. 166, xxxv. 453).

7 Annal. d. Chem. und Pharm. (Suppl.) iv. 50 ; cxl. 145, 1866 ; exlix. 35, 1869 (Amer. Journ. Pharm. xxxviii. 568, xxxix. 122, xlii. 396).

8 Repert. f. Pharm. xxxi. 37.

9 Annal. d. Chem. und Pharm. xlviii. 109, 1843; lv. 105, 1845 (Pharm. Journ. and Trans. [1], iii. 177 ; v. 228).

10 Vierteljahresschr. f. pract. Pharm. viii. 126, 1859.

11 Ibid. ix. 489, 1860 ; Annal. d. Chem. und Pharm. cxxi.372, 1862 (Amer. Journ. Pharm. xxxii. 450, xxxiii. 122, xxxiv. 406).

12 Annal. d. Chem. und Pharm. (Suppl.) iv. 40. Müller's alstonine from Alstonia constricta is probably a mixture of these two alkaloids (Hesse).

${ }^{13}$ Archiv d. Pharm. xlix. 153, 1847. Vierteljahresschr. f. pract. Pharm. viii. 536, 1859; ix. 524, 1860. Annal. d. Chem. und Pharm. cxxxvii. 274, 1866. (See Bentley, Pharm. Journ. and Trans. [2], iv. 343, 1862 ; Amer. Journ. Pharm. xxxiii. 112).

${ }^{14}$ Chem. Centralblatt, 781, 1865 ; and N. Jahrb. f. Pharm. xxxi. 193, 1869 (Pharm. Journ. and Trans. [3], i. 682, 1871). 
Ditamine (echitamine), see Gorup Besanez, and Hesse ${ }^{1}$ for ditaïne see Harneck. ${ }^{2}$ The latter is glucosidal, like solanine, and assumes a flesh-colour when treated with conc. sulphuric acid, whereas ditamine turns splendid purple.

Geissospermine and aspidospermine, compare Fraude. ${ }^{3}$ The latter yields a deep violet solution when warmed with excess of perchloride of platinum; heated with dilute sulphuric acid and a little chlorate of potash, or with perchloric acid of sp. gr. $1 \cdot 13$, it turns deep red; with sulphuric acid and peroxide of lead, brown, changing to cherry-red. If not quite pure, in the latter case a violet colour is produced. At a temperature of $14^{\circ}$ aspidospermine dissolves in 6,000 parts of water, 48 of 98 per cent. spirit, and 106 of ether.

Dulcamarine, see Wittstein $;^{4}$ alkaloid in Eschscholtzia, see Walz; ${ }^{5}$

1 Annal. d. Chem. und Pharm. clxxvi. 88, 326 ; clxxviii. 49, 1875 (Pharm. Journ. and Trans. [3], vi. 142, 1875). Ber. d. d. chem. Ges. xiii. 1841, 1880 (Year-book Pharm. 171, 1881).

${ }^{2}$ Archiv f. exper. Pathol. und Pharmacol. vii. 128, 1877. Ber. d. d. chem. Ges. xi. 2004, 1878 (Year-book Pharm. 188, 1878); ibid. xiii. 1645, 1880 (see also Pharm. Journ. and Trans. [3], viii. 803, 1878; xi. 331, 1870). Scharlée's alstonine (Hesse's alstonanine) from Alstonia spectabilis appears to be closely allied to ditamine, but crystallizes with facility.

${ }^{3}$ Ber. d. d. chem. Ges. xi. 2189, 1878 (Year-book Pharm. 193, 1879); ibid. xii. 1558, 1560 (Pharm. Journ. and Trans. [3], x. 712, 1880). See also my observations in the Jahresb. f. Pharm. 120, 1878; and Hesse, ibid, 115, 1877 (Pharm. Journ. and Trans. [3], viii. 648, 1878). The name geissospermine appears to have been applied to two different alkaloids, of which the one discovered by Hesse yields reactions closely resembling those of aspidospermine (red colouration with nitric acid, etc.). Hesse's geissospermine produces a splendid red colour with sulphuric acid and bichromate of potassium, blue with sulphuric acid and ferric salts, deep blue with Fröhde's reagent, and changes the colour of chloride of gold solution to a deep red. It can be removed from solution by shaking with benzene or chloroform, and is accompanied by an alkaloid which is easily soluble in ether and turns reddishviolet with sulphuric acid. The identity of aspidospermine and paytine already alluded to $(\S 186)$ is contested by Hesse. The same chemist has also lately discovered a second alkaloid in quebracho, which he calls quebrachine; it is coloured blue with sulphuric acid and peroxide of lead (Ber. d. d. chem. Ges. xiii. 2308; see Pharm. Journ. and Trans. [3], xii. 704). In examining quebracho bark, I noticed that chloroform extracted from acid solutions (\$ 55) a small quantity of an alkaloid giving the reaction of aspidospermine. Solutions rendered alkaline with ammonia yielded to petroleum spirit and benzene a mixture that reacted like aspidospermine with sulphuric acid and chlorate of potash, but was coloured splendid violet by Fröhde's reagent, and behaved like strychnine to sulphuric acid and bichromate of potash. Compare also Arata, Actas de la Acad. nac. in Buenos Aires, 1881 ; Hesse, Annal. d. Chem. u. Pharm. cexi. 249, 1882.

${ }^{4}$ Pharm. Vierteljahresschr. i. 371, 495, 1850. Cf. § 167.

5 N. Jahrb. f. Pharm. viii. 223, 1857 (Amer. Journ. Pharm. xxxiv. 329). Compare also my 'Ermittelung d. Gifte.' 
glancine, see Probst;1 fumarine, see Pommier, Hannon, and Preuss; ${ }^{2}$ gelsemine, see Robbins ${ }^{3}$ (cf. $\$ 555,171$ ); hydrastine, see Perrins ; jurubebine, see Greene ; ${ }^{5}$ loturine, see $\S 168$; menispermine and paramenispermine, see Szteyner $;^{6}$ oleandrine, see Leukowsky $;^{7}$ oxyacanthine, see Polex $;^{8}$ pelletierine (punicine), see Tanret. ${ }^{9}$

Pereïrine, see Goos. ${ }^{10}$ It dissolves with purple colour in nitric acid.

Sparteïne, see Mills (cf. § 55) ; il taxine, see Marmé (cf. §§ 55, 171) ; ${ }^{12}$ lycopodine, ${ }^{13}$ nupharine. ${ }^{14}$

$\S 190$. Amides.-The following are amides of less complex constitution occasionally met with in plants :

Amanitine, which 'may be distinguished from muscarine by the properties of the gold salt $(\S 183)$ and by the negative results of physiological experiments. ${ }^{15}$ It is isomeric, but not identical with choline (neurine, sinkaline), and is converted into muscarine by the action of nitric acid, whereas, under similar conditions, choline yields betaïne (= butylalanine and oxyneurine). Muscarine differs from betaïne in being more powerfully alkaline. ${ }^{16}$

1 Annal. d. Chem. und Pharm. xxix. 120, xxxi. 250, 1838 (Amer. Journ. Pharm. xxxiii. 9).

${ }^{2}$ N. Repert. f. Pharm. ii. 469, 1853 ; Vierteljahresschr. f. pract. Pharm. iii. 68,1852 ; Zeitschr. f. Chem. ii. 414, 1866.

3 Jahresb. f. Pharm. 152, 1876 (Amer. Journ. Pharm. 191, 1876). Robbins identified the so-called gelsemic acid with aesculin.

${ }^{4}$ Pharm. Journ. and Trans. [2], iii. 546, 1862. See also Mahla, Journ. f. pract. Chem. xci. 248 ; Prescott, Amer. Journ. Pharm. xlvii. 481, 1875 ; Hale, ibid. 247.

5 Amer. Journ. Pharm. xlix. 506, 1877.

6 Jahresb. f. Pharm. 141, 1878.

7 Ibid. xlvi. 397.

${ }^{8}$ Archiv d. Pharm. [1], vi. 271, 1824 (Amer. Journ. Pharm. xxxiii. 455).

9 Journ. de Pharm. et de Chimie, xxviii. 168, 1878 (Pharm. Journ. and Trans. [3], ix. 450).

${ }^{10}$ Chem. Centralblatt, 610, 1839. Compare also Peretti, Journ. de Chim. med. xxvi. 162.

11 Annal. d. Chem. und Pharm. cxxv. 71, 1862.

12 Jahresb. f. Pharm. 93, 1876 ; compare also 636, 1878 (Pharm. Journ. and Trans. [3], 893, 1877).

13 Annal. d. Chem. und Pharm. ccviii. 363, 1881 (Pharm. Journ. and Trans. [3], xii. 280,1881$)$.

${ }^{14}$ Grüning, loc. cit.

${ }^{15}$ Compare Schmiedeberg, Ber. d.d.chem. Ges. iv. 693, 1871, and Harnack, loc. cit. (Pharm. Journ. and Trans. [2], xi. 365, 1869).

${ }^{16}$ For betaïne see Scheibler, Ber. d. d. chem. Ges. ii. 292, 296, 1869 ; identity with oxyneurine, ibid. iii. 155; with lycine see Husemann, Schweiz. 
Choline and amanitine are also tolerably strongly alkaline. The double salt of platinum and choline is precipitated by alcohol from aqueous solution (contains $31 \cdot 75$ to 33.27 per cent. $\mathrm{Pt}$.) ; the gold double salt is sparingly soluble in cold water, more freely in boiling (contains 44.25 to 44.9 per cent. Au.). The gold and platinum double salts of betaine are freely soluble in water, and especially so in alcohol, but more sparingly in ether.

$\S 191$. Asparagine.-This substance requires 40 parts of cold and 4 of warm water for solution, and is insoluble in absolute alcohol and in ether. It crystallizes in colourless rhombic prisms. Boiling with hydrochloric acid resolves it into aspartic acid and ammonia, a reaction upon which Sachsse ${ }^{1}$ based the following method for the quantitative estimation :-10 grams of the powdered substance are boiled for a quarter of an hour with $200 \mathrm{cc}$. of a mixture of equal volumes of alcohol and water, in a flask provided with an upright condenser; 5 cc. of a cold saturated alcoholic solution of mercuric chloride are diluted with $5 \mathrm{cc}$. of water, and added to the mixture whilst still hot, the whole thrown on a filter, and washed first with hot 50 per cent. spirit, and finally with cold water. The filtrate and washings are evaporated to dryness, the residue redissolved in the smallest possible quantity of water (not more than $50 \mathrm{cc}$.), from which solution the mercury is precipitated with sulphuretted hydrogen. The sulphide of mercury is filtered off and washed with hot water until filtrate and washings measure 110 to $120 \mathrm{cc}$. This liquid is then mixed with 10 cc. of hydrochloric acid and boiled for an hour (with upright condenser), by which the asparagine is decomposed into ammonia and aspartic acid; it is then cooled in an atmosphere free from ammonia, and made slightly alkaline with pure potash. The ammonia produced may be estimated gasometrically by Knop's method; 14 parts by weight of nitrogen indicate 132 of anhydrous asparagine. For asparagine, see also $\$ \S 97,210$.

The microscopical detection of asparagine may be effected by taking advantage of its insolubility in absolute alcohol. Crystalline deposits of that substance are usually formed when fresh sections of plants containing it are placed in alcohol. After being dried they are insoluble in a cold saturated aqueous solution of

Wochenschr. 1875 (Amer. Journ. Pharm. 209, 1875). Compare also Annal. d. Chem, und Pharm. (Suppl.) ii. 383, iii. 245, 1864.

1 Journ. f. pract. Chem. vi. 118, 1873 (Journ. Chem. Soc. xxvi. 652). 
asparagine, and melt at $100^{\circ}$ to a homogeneous mass easily soluble in water. Should the addition of alcohol not be followed by the immediate formation of crystals, Borodin recommends covering the object with a coverslip, allowing the spirit to evaporate, and then again looking for crystals.

Schulze and Ulrich detected glutamine in beet-juice by precipitating with a slight excess of basic acetate of lead and filtering. To the filtrate which contains the glutamine, hydrochloric acid is added in the proportion of $25 \mathrm{cc}$. to a litre; on boiling for two hours the glutamine is decomposed, like asparagine, into ammonia and the corresponding acid (glutamic acid). The greater part of the hydrochloric acid is now removed by concentrated solution of acetate of lead, and to the filtrate basic acetate of lead is added until the precipitate first formed is redissolved, with the exception of the remainder of the chloride of lead. The lead salt of glutamic acid is then precipitated from the filtered solution by the addition of alcohol. It is collected, decomposed with sulphuretted hydrogen, and filtered from the sulphide of lead. After expelling the-excess of sulphuretted hydrogen from the liquid, oxide of silver is added to remove any hydrochloric acid present, the solution freed from silver by sulphuretted hydrogen, and evaporated to crystallization. The glutamic acid thus obtained may be purified by conversion into a copper salt and regeneration by sulphuretted hydrogen. The presence of glutamic acid was confirmed by converting with nitrous acid into the corresponding oxy-acid, from which pyrotartaric acid was obtained by the action of hydriodic acid.

The last portion of glutamic acid from the crystallization of the crude product was found to contain aspartic acid. ${ }^{1}$

Glutamine may be estimated quantitatively in the same manner as asparagine.

For the quantitative determination of asparagine, leucine, tyrosine, etc., see also $§ 241$.

$\S 192$. Leucine.-This substance has also been detected in

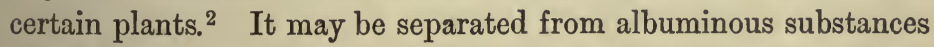
by dialysis, and if present in solution with asparagine, will be found in the mother-liquor after the crystallization of the latter.

${ }^{1}$ Compare Zeitschr. f. anal. Chem. xvii. 104, 1878.

2 Compare, for instance, Gorup Besanez, Ber. d. d. chem. Ges. vii. 146, 569, 1874 (Journ. Chem. Soc. xxvii. 494). 
It is characterized by crystallizing in sphæro-crystals, by its behaviour to water and alcohol (dissolves in 27 parts of cold, and easily in warm water, in 1040 of cold 96 per cent. spirit, and 800 of boiling 98 per cent.), by its power of dissolving oxide of copper, and by yielding leucic acid when acted upon by nitrous acid.

I have already directed attention to the identity of the chenopodine obtained from decomposing yeast with leucine. ${ }^{1}$ Gorup Besanez has made the same assertions of the chenopodine from Chenopodium album.

Tyrosine, as well as leucine, has lately been detected in plants, especially in germinating seeds. ${ }^{2}$ Aqueous extracts of the material under examination are concentrated, precipitated with alcohol, filtered, freed from alcohol by distillation, evaporated to a syrup, and allowed to stand, when the tyrosine separates out in groups of warty crystals. By recrystallization from ammoniacal alcohol it may be obtained in acicular crystals. Solutions of the latter develop a rose-colour with mercuric nitrate and a little nitrous acid. Warmed to $50^{\circ}$ with concentrated sulphuric acid (half-hour), and saturated with carbonate of barium, it yields a mass which assumes a fine violet with ferric chloride.

Ratanhin agrees with tyrosine in most of its reactions, especially the two just described. It is almost insoluble in cold water, alcohol and ether, sparingly soluble in boiling water, but freely in ammonia. Suspended in water, and then warmed with a little nitric acid, it dissolves, passing, as it does so, from rose to rubyred and blue, turning finally green with red fluorescence. ${ }^{3}$

\section{VEGETABLE MUCILAGE.}

$\S$ 193. Vegetable Mucilage, Pectin, etc.-The substances designated by these names are a source of much inconvenience to the analyst, due probably to their occurring in various modifications

${ }^{1}$ Compare Bergman, 'Das putride Gift,' Dorpat Gläser. See also Reinsch, N. Jahrb. f. Pharm. xxvii. 123, 1867.

${ }^{2}$ Compare Schulze and Barbieri, Ber. d. d. chem. Ges. x. 199, xi. 710 (Journ. Chem. Soc. xxxiv. 663).

${ }^{3}$ It is notorious that ratanhin does not occur in rhatany-root, and that its presence in certain samples of commercial extract of rhatany is due to adulteration. Compare also Kreitmair, Jahresb. f. Pharm. 136, 1874 (Year-book Pharm. 90, 1875). Gintl believes ratanhin to be identical with angelin from Ferreira spectabilis (Zeitschr. d. österr. Apoth. Ver. 32, 1869). 
differing greatly from one another in solubility, etc. The majority of them are characterized by a certain disposition to combine with lime, potash, etc., and this has led to their classification with the weak organic acids (arabic acid, etc.); in fact, some of the differences in solubility, etc., seem occasionally to depend directly upon the quantity and quality of the bases with which they are combined. 1

But other substances, such as albumen, tannin, etc., that simply accompany the pectin and mucilage, can also exercise an influence on the behaviour of the latter to solvents. On this account, and because, as a rule, such matters as mucilage diffuse but slowly, it is not always possible, in extracting vegetable substances with water according to $\S 71$, to be certain that all the mucilage (arabin, etc.) soluble in water has really passed into solution. If heat were employed, the amount dissolved would certainly be increased, but at the same time other and more serious errors would be introduced.

One such source of error is to be found in the presence of carbohydrates closely allied to soluble mucilage, but differing from it in only swelling (not dissolving) in cold water (metarabic acid, etc.). These carbohydrates are of frequent occurrence in plants. Prolonged heating with water gradually dissolves them. Error would also be caused by carbohydrates like lichenin, caraghin, starch, etc., hot solutions of which gelatinize on cooling, as well as by other substances.

$\S$ 194. Modified Method of Examination for Mucilage, etc.-For the reasons given in $\S 193$, I think it is preferable to extract with cold water, and estimate the mucilage and albumen in the solution prepared according to $\S 71$. The washings mentioned in that section should be evaporated to a syrup, in which a similar determination of mucilage and albumen should be made. If the material has been macerated with exactly $100 \mathrm{cc}$. of water, and 65 cc. of filtrate have been obtained (used for the first determination of albumen, etc.), then 35 cc. must be retained by the residue and filter, and these $35 \mathrm{cc}$. must be extracted by the washing. If the

1 This was clearly the case in peony-seed (Archiv d. Pharm. [3], xiv. 426, 1879 ; Journ. Chem. Soc. xxxv. 1043). Treatment of the seed with alcoholic tartaric acid rendered far more arabic acid soluble in water than it was possible to extract by direct treatment with the latter menstruum. It had evidently been liberated from combination by tartaric acid, and rendered soluble in water. 
extraction has been complete, the amount of albumen, etc., in the washings must be the same as that in 35 cc. of the first filtrate (which may be calculated from the first estimation); but should the washings contain more than the corresponding quantity of filtrate, the excess must be added to the total of the analysis.

$\S 195$. Characters of Soluble Mucilage.-Apart from their being dissolved by water and precipitated by alcohol from aqueous solution, vegetable gum, arabin, arabic or gummic acid, is also characterized by being converted into glucose when boiled with a dilute acid. It must, however, be observed, that the various arabic acids, according to their origin, yield glucoses differing to a certain .extent from one another, some being more powerfully dextro-, others lævo-rotatory; some crystallizing with facility, others again not at all, or only with difficulty, or passing first through an intermediate stage as dextrin (according to Kirchner with simultaneous production of cellulose). By means of these properties, vegetable mucilages of particular origin can sometimes be accurately described. Some years ago it was shown by Scheibler ${ }^{1}$ that the arabic acid of beet-root yielded, on inversion, a considerable quantity of dextro-rotatory arabinose, which crystallizes with such facility that it was at first thought to be mannite.

Kiliani has recently asserted the identity of arabinose with lactose. Many varieties of gum arabic also behave like arabic acid, whilst some which are not otherwise distinguishable from good gum, differ in yielding lævo-rotatory non-crystallizable glucose. In addition to these, Béchamp ${ }^{2}$ has recently discovered a 'gummicose' which appears to be allied to galactose (\$205). I am almost inclined to think that a minute investigation of these properties might enable us to distinguish between the various forms of vegetable mucilage soluble in water. ${ }^{3}$

The examination of the oxidation-products obtained by the

${ }^{1}$ Ber. d. d. chem. Ges. vi. 612, 1873 ; Journ. f. pract. Chem. ciii. 458, 1868 (Journ. Chem. Soc. xxvi. 1124). See also Neubauer, Jahresb. f. Pharm. 6, 1854, and Græger, ibid. 218, 1872.

${ }^{2}$ Compare Béchamp, Journ. de Pharm. et de Chim. xxvii. 51, 1878.

${ }^{3}$ In general it may be said that the action of dilute acids must be continued for a longer time to convert vegetable mucilage (arabin) into glucose than is necessary for dextrin, triticin, etc. But it must be left for further experiments to show to what extent the amount of glucose produced may be taken as an indication of the quantity of arabin originally present. 
action of nitric acid may also result in the discovery of distinctive properties. Special attention should be directed to the presence among these oxidation-products of mucic acid, and to the quantity in which it is yielded.

The action of aqueous solutions of these mucilaginous substances on polarized light also requires further investigation. It is at all events certain that some arabins are powerfully lævorotatory (cf. $§ 146$ ), others feebly so, whilst some again are dextrorotatory.

By the action of hydrochloric, or tolerably dilute sulphuric acid, or spirit containing 10 per cent. of the latter, arabic acid is converted into metarabic acid ( $\$ 226$ ) which is characterized by only swelling in water. This modification can be converted into arabic acid by boiling with a very dilute non-oxidizing acid, a little sugar being simultaneously produced. A similar change to arabic acid takes place when metarabic acid is triturated with sufficient lime or baryta water to dissolve it, a lime or baryta salt of arabic acid being formed.

Arabic acid agrees in its essential properties with metapectic acid, and approaches pectic acid in such a manner as to allow of the conjecture that the latter, when examined in a state of purity, will prove identical with it; in fact, I am of the same opinion as Reichardt ${ }^{1}$ and others-viz., that the so-called pectin substances are nothing else than the various forms of vegetable mucilage and its nearest allies.

The insolubility of these mucilaginous and 'pectinous' substances in alcohol and ether, and the property they possess of swelling in contact with water, allow of their detection microscopically. They are generally coloured yellow by iodine water (for allied substances coloured blue with iodine, see $\S 244$ ). Aniline violet colours vegetable mucilage red.

$\S 196$. Vurieties of Gum Arabic.-Masing ${ }^{2}$ has published communications on the behaviour to reagents (\$73) of arabic acid, and different varieties of gum arabic, and its more important surrogates. It' appears that 10 per cent. aqueous solutions of these substances are not precipitated by cold saturated solution of acetate of copper, 10 per cent. acetate of lead solution, or by ferric

${ }^{1}$ Archiv d. Pharm. [3], x. 116, 1877 (Journ. Chem. Soc. xxxii. 502).

${ }^{2}$ Archiv d. Pharm. [3], xv. 216, 1879; xvii. 34, 1880 (Year-book Pharm. 191, 1881 ; Journ. Chem. Soc. xl. 212). 
chloride of sp. gr. 1.2 ; a cloudiness or precipitate produced in some samples (e.g. Feronia elephantum) is probably due to contamination. Silicate of potash (1 part of thick soluble glass diluted with 20 parts of water) produces in solutions of gum arabic, and most of its surrogates, a cloudiness or precipitate which partially or wholly redissolves on adding excess. Arabic acid remains clear, or becomes only slightly turbid. The same reagent does not precipitate the partially soluble gum from species of Cactus, Cedrela, or Rhizophora, or solutions of the gum from Acacia catechu, A. leucophloea, and species of Albizza, Azedirachta, Odina and Conocarpus. A 2 per cent. solution of stannate of potash yields reactions resembling in general those of silicate of potash, but produces in solutions of arabic acid a precipitate that redissolves in excess. A 10 per cent. solution of neutral sulphate of aluminium gives, as a rule, a precipitate, and this is in many cases soluble in caustic potash of sp. gr. 1.13. Basic acetate of lead also produces a precipitate, which is generally partially or wholly soluble in excess.

$\S 197$. Separation from Dextrin, etc.-The behaviour of the mucilaginous substances soluble in water to basic acetate of lead furnishes us with the means of getting rid of them, should we wish to make optical or chemical experiments with extracts of vegetable substances for the detection of glucose, saccharose, dextrin, triticin, etc. (cf. $\S 76,83$ ); care must be taken, however, not to add any large excess of the precipitant. If this precaution is observed, such substances as arabin and dextrin can, I think, be more completely separated than by the precipitation with alcohol, recommended in $\$ \$ 75,76$. In the latter, ethylic alcohol can, as I showed some years since, be replaced by methylic. ${ }^{1}$

\section{DEXTRIN, TRITICIN, SINISTRIN, LEVULIN.}

§ 198. Characters.-These carbohydrates are all easily converted into glucoses $(\S 76)$ by dilute acids. Dextrin may be distinguished by its yielding grape-sugar, whilst triticin, sinistrin and levulin are converted into levulose, and are also characterized by their behaviour to baryta-water (see also $\S 77$ ). Levulin ${ }^{2}$ is optically

1 Pharm. Zeitschr. f. Russland, iv. 152, 1866 (note).

2 Compare Weyher v. Reidemeister, 'Beitr. z. Kenntniss d. Levulins, etc.' Diss. Dorpat, 1880. 
inactive; sinistrin and triticin ${ }^{1}$ differ from one another in the extent to which they deviate a ray of polarized light. Triticin reduces Fehling's solution rapidly, but levulin and sinistrin require long boiling before cuprous oxide separates out (levulin, $1 \frac{1}{2}$ hour). These three carbohydrates also differ in the rapidity with which they are converted into glucose when heated with pure water in sealed tubes. Triticin is here the first to undergo a partial transformation into sugar. Levulin is more easily converted by yeast into carbonic acid and alcohol than is either triticin or sinistrin.

$\S$ 199. Alcoholates of the foregoing Carbohydrates, Composition, Quantitative Estimation, etc.-The precipitates of levulin, triticin and sinistrin produced by spirit retain notable quantities of alcohol in such a manner as to necessitate the inference that distinct alcoholates have been formed. Even after standing for three months over sulphuric acid the levulin compound had not lost all its alcohol, whilst in a partial vacuum it had disappeared in the course of two months. Prolonged heating to $110,^{\circ}$ a temperature at which the alcoholate melts, is sufficient to cause the gradual expulsion of the alcohol.

In estimating the four carbohydrates in question by calculation from the amount of sugar yielded by the action of dilute acids, it must be remembered that the composition of dextrin, sinistrin and levulin, dried at $100^{\circ}$, has been found by analysis to correspond to the formula $\mathrm{C}_{6} \mathrm{H}_{10} \mathrm{O}_{5}$, but that triticin dried at $110^{\circ}$ has the same composition as cane-sugar : viz., $\mathrm{C}_{12} \mathrm{H}_{22} \mathrm{O}_{11}$.

In these cases the glucose $(\S 76)$ is better estimated by Fehling's solution ( $\$ 83$ ) than by polarization ( $\$ 208)$. Solutions of levulin, triticin and sinistrin, although inverted with dilute acids only, do not always show an angle of rotation $=106^{\circ}$ to $107^{\circ}(\S 205)$, even if the solution be neutralized, evaporated to dryness, redissolved in cold water, and tested immediately. On cooling the solution after inversion and examining at once, levulin yielded, in the most favourable instances, a sugar with a rotatory power of $81^{\circ}$, triticin, $94^{\circ}$, and sinistrin, $96^{\circ}$; and it was only by conducting the inversion in sealed tubes that Reidemeister obtained for triticin-sugar a rotation of $106.5^{\circ}$, indicating the presence of pure levulose. ${ }^{2}$

${ }^{1}$ For mycodextrin and mycinulin see Ludwig and Busse, Archiv d. Pharm. elxxxix. 24, 1869.

${ }^{2}$ Compare Reidemeister, loc. cit. 
For the complete inversion of 1 to 1.3 grams of carbohydrate dissolved in 35 cc. of water, 5 to 6 drops of hydrochloric acid (33 per cent.) were found sufficient; in the case of levulin, two to two and a half hours' boiling is required, whilst triticin is completely converted in twenty-five to thirty minutes (Reidemeister).

To obtain accurate results the acid must be as dilute as possible, and the action stopped as soon as the inversion is complete. Here, as in many other instances, hydrochloric acid seems to be preferable to sulphuric; considerable quantities of sugar (20 to 30 per cent.) may otherwise be lost by secondary decompositions, and in consequence the estimation of the carbohydrate be attended with results inaccurate in the extreme.

For the estimation of glucose by titration, see $\$ \S 83,84$.

For dextrin, see also $\S \S 200,201,202$.

\section{GLUCOSES, SACCHAROSES, ETC.}

$\S 200$. Detection of Grape-sugar.-To detect grape-sugar (\$ 70, 83 to 88 ), Mulder ${ }^{1}$ makes use of its reducing action on indigo. The solution to be examined is rendered faintly blue with sulphindigotic acid; carbonate of soda is then added until the reaction is alkaline, and the whole boiled for a few seconds. If grape-sugar is present, the colour passes from blue to violet, and then disappears ; the liquid should not, however, be shaken, as the action of the air results in the rapid return of the blue colour. ${ }^{2}$

Tincture of litmus, substituted by $\mathrm{Vogel}^{3}$ for indigo, is less sensitive.

Braun ${ }^{4}$ directs attention to the reaction between glucose and picric acid, and recommends this reagent for distinguishing between grape- and cane-sugar. In the presence of caustic soda picric acid is converted by boiling with grape-sugar into blood-red picramic acid. Fruit- and milk-sugar exert a similar action, which is not shared by mannite or cane-sugar.

For the action of grape-sugar on ferridcyanide of potassium, see Gentele ${ }^{5}$ and Lenssen. ${ }^{6}$ The latter also discusses the reduc-

${ }^{1}$ Chem. Centralblatt, 176, 1861 (Pharm. Tourn. and Trans. [1], xviii. 421).

${ }^{2}$ Compare Neubauer, Zeitschr. f. anal. Chem. i. 378, 1862.

${ }^{3}$ N. Repert. f. Pharm. xi. 62, 1862.

4 Zeitschr. f. anal. Chem. iv. 185, 1865.

${ }^{5}$ Chem. Centralblatt, 91, 1861 ; Stahlschmidt, Ber. d. d. chem. Ges. 141, 1861 (Amer. Journ. Pharm. xxxii. 81).

6 Zeitschr. f. anal. Chem. ix. 453, 1870. 
tion of cyanide of mercury by glucose as recommended by Knapp ${ }^{1}$ $(\S 84)$, and shows that by neither method can saccharose be accurately distinguished from glucose.

O. Schmidt distinguishes between grape- and cane-sugar by adding basic acetate of lead and ammonia, and warming. The white precipitate that first appears turns red if grape-sugar is present ; cane-sugar does not yield this reaction. ${ }^{2}$

$\$ 201$. Estimation of Glucose in Presence of Saccharose. - In titrating glucose in the presence of saccharose, it is advisable, according to Rumpf and Heinzerling, ${ }^{3}$ to conduct the experiment with the utmost rapidity, as the error caused by the dextrin is then but very small.

Barfoed detects grape-sugar in the presence of dextrin by means of an aqueous solution of crystallized acetate of copper ( 1 in 15), containing 1 per cent. of acetic acid. To the liquid under examination a few drops of the reagent are added, the whole boiled for an instant and cooled. If grape-sugar is present, cuprous oxide gradually separates out.* Cane-sugar, milk-sugar, and gum behave like dextrin. These statements have been confirmed by Müller. ${ }^{5}$

$\S 202$. Detection of Dextrin in Presence of Cane-sugar.-Scheibler ${ }^{6}$ has shown that a 20 per cent. aqueous solution becomes cloudy on the addition of 4 volumes of 90 to 95 per cent. alcohol if the sugar is contaminated with as little as even .5 per cent. of dextrin. A second test of Scheibler's, depending upon the use of iodine, may be passed over here, as it succeeds with impure dextrin only. The presence of dextrin and cane-sugar in solution together may often be inferred if, after inversion, Fehling's solution shows less grape-sugar than could have been anticipated by calculation from the rotatory power before inversion; or if the rotatory power after inversion is less than that before inversion (taken as due to pure cane-sugar) would have led us to expect. (See $\S 207$.)

\$203. Gravimetric Estimation of Glucose.-Mulder recommends

1 Annal. d. Chem. und Pharm. cliv. 252 (Pharm. Journ. and Trans. [3], i. $301,1870)$.

${ }^{2}$ Annal. d. Chem. und Pharm. cxix. 102, 1861.

${ }^{3}$ Zeitschr. f. anal. Chem. ix. 358, 1870. Compare also Barfoed, ibid. xii. 29, 1873 (Year-book Pharm. 176, 1874).

${ }^{4}$ Zeitschr. f. anal. Chem. xii. 27, 1873 (Journ. Chem. Soc. xxvi. 1163).

${ }^{5}$ Zeitschr. f. anal. Chem. xviii. 601 (Year-book Pharm. 74, 76, 1879).

${ }^{6}$ Zeitschr. f. anal. Chem. x. 372, 1871. 
for this purpose heating for an hour to $60^{\circ}$ with excess of alkaline copper solution, filtering off, and weighing the cuprous oxide precipitated ( $(83)$. The errors involved by this method, which at the best of times can yield only approximate results, have been the subject of communications by Fresenius ${ }^{1}$ and Gratama. ${ }^{2}$

$\S 204$. Fermentation Test $(\S 85)$. - To detect glucose by this test a little of the suspected solution is evaporated until it contains at least 5 per cent. of sugar. After cooling a few drops of tartaric acid solution are added, and a little good yeast, previously washed with distilled water; 1-2 cc. of this mixture are then introduced into a eudiometer standing over mercury. If glucose is present

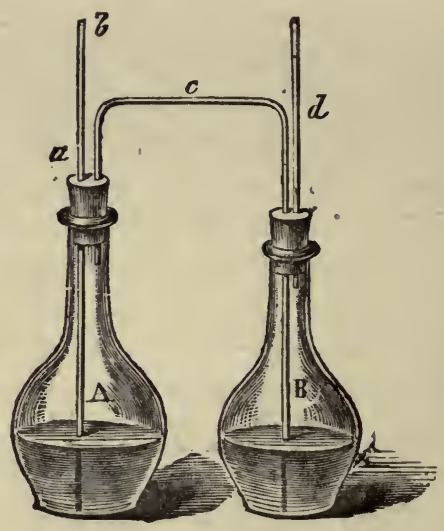

Fig. 9.

an evolution of carbonic acid will soon begin, and should, in a few hours, displace a considerable amount of mercury ( $\$ 61)$. By means of a blank experiment with distilled water, and the same quantity of yeast, it may be ascertained whether any carbonic acid has been produced by the yeast itself alone.

The absence of any evolution of carbonic acid is not necessarily a proof that the liquid is free from glucose. Certain constituents of plants, such as salicylic acid, thymol, etc., may occasionally be present, and prevent any fermentation taking place. ${ }^{3}$

1 Anleit. zur quant. Analyse, 5th edition, 833.

2 Zeitschr. f. anal. Chem, xvii. 1875, 1878 (Journ. Chem. Soc. xxxiv. 611).

3 Compare Werncke, 'Ueber die Wirkung einiger Antiseptica auf Hefe.' Diss. Dorpat, 1879. 
If this is the case, such a method cannot of course be employed for the quantitative determination of the glucose. But if the solution soon passes into a state of fermentation, and a tolerably large quantity of gas is produced, an estimation of the amount of glucose present may be made in the following manner: The glucose solution is prepared as directed for the qualitative test, and a measured quantity of it introduced into the flask A (Fig. 9). The second flask B, shown in the figure, contains sulphuric acid, as for the determinaiion of carbonic acid according to the method of Fresenius and Will. The whole apparatus is wiped dry, accurately weighed, and exposed to a temperature of $20^{\circ}$ to $30^{\circ}$. The carbonic acid evolved in A, passes through $c$, and is dried by the sulphuric acid in $\mathrm{B}$, before being expelled through $d$. After the lapse of about two days, when the evolution of gas has ceased, the carbonic acid in the apparatus is driven out by aspirating at $c_{\text {. }}$ The difference in weight is the amount of carbonic acid produced, 100 parts of which are equivalent to 204.54 of glucose. ${ }^{1}$

$\S 205$. Characters of the Various Glucoses. - In speaking of glucoses in the preceding paragraphs, I referred principally to grape-sugar, fruit-sugar, and the combination of both known as invert-sugar. Grape-sugar (dextrose) possesses the following characters : it can be obtained, by gradual deposition from solution, in prismatic crystals $^{2}$ containing one molecule of water (cf. $§ 89$ ); an aqueous solution, prepared fresh and without heat, in which the sugar is present in the crystalline modification, shows a rociatory power for $(\alpha)_{\mathrm{D}}+91.81^{\circ}(p=1)$; whereas a solution that has been heated, in which the sugar has passed into the amorphous condition, possesses for $(\alpha)_{D}$ a rotatory power of $49.54^{\circ}$ to $50.00^{\circ}$, and $46.34^{\circ} 3(p=12)$. Hoppe Seyler ${ }^{4}$ found the rotatory power of amorphous grapesugar to be $(\alpha)_{D}=56.4^{\circ}$, from which he obtained the constant quantity $\mathrm{A}^{\prime} \mathrm{D}=1773.0$; this may be used for calculating the

1 Another method that has been suggested of estimating glucose consists in determining the speciic gravity before and after fermentation (for 20 to 24 hours) and calculating 0.219 per cent. of glucose for each 0.001 in the difference. Manasseïn has shown that the method yields good results with the sugar in urine (Med. Centralblatt, 551, 1872).

${ }^{2}$ Similar crystalline deposits, especially sphærocrystals, have been occasionally observed in the microscopic examination of dried drugs. Compare Braun, Zeitschr. d. öesterr. Apoth. Ver. xvi. 337, 1878.

${ }^{3}$ Compare Hesse, Annal. d. Chem. und Pharm. clxxvi, 89, 1875 (Pharm. Journ. and Trans. [3], vii. 191 et seq.).

4 Zeitschr. f. anal. Chem. xiv. $303,1875$. 
amount of grape-sugar present from the rotation observed. For anhydrous dextrose Tollens and v. Grote ${ }^{1}$ ascertained $(\alpha)_{D}=53 \cdot 10^{\circ}$, and the constant quantity $=1883 \cdot 3$. Hesse has shown that the prolonged heating of aqueous solutions of dextrose reduces the angle of rotation still further.

Biot has asserted the existence of glucoses, agreeing with grapesugar in all important properties, but differing from it in their rotatory power; and the truth of this assertion has been proved by Hesse. An instance may be cited in salicin-sugar, the crystalline modification of which possesses a rotatory power $(\alpha)_{\mathbf{D}}=100^{\circ}(p=1)$, and the amorphous $(\alpha)_{\mathrm{D}}=50^{\circ}$ (populin-sugar is said to correspond to ordinary dextrose); the rotatory power of phlorose from phloricin is five-sixths that of glucose. ${ }^{2}$

Fruit-sugar (levulose) has recently been obtained in acicular crystals $;^{3}$ a freshly prepared solution shows a rotatory power $(\alpha)_{\mathbf{D}}=-106^{\circ}$ (constant 943.4 ; see also note to $\$ 209$ ), which slowly diminishes, on boiling, to $-56^{\circ}$. Levulose dissolves more easily in alcohol than crystalline dextrose, which may therefore, to a certain extent, be freed from the former by washing with spirit. The disposition to form a sparingly soluble compound with lime is very characteristic of levulose, and forms, at the same time, a means of separating this from grape and other sugars. Like dextrose, levulose is fermentable; but when both sugars are present together, the former is the first to undergo alcoholic fermentation.

Sugars allied to levulose, differing from it only in the rotatory power, will probably be found in certain plants (Jerusalem artichokes, etc. ; see also $§ 199)$.

Invert-sugar (see also $§ 209$ et seq.) shows as a rule a rotatory power for $(\alpha)_{\mathbf{D}}$ of $-18^{\circ}$. The majority of chemists regard it as a mixture of grape- and fruit-sugar, molecule for molecule; but Mauméné ${ }^{4}$ differs from them, and maintains that he has found in it, in addition to these two constituents, another, optically inactive, unstable sugar.

1 Ber. d. d. chem. Ges. 487, 616, 1531, 1876. See also Hesse, Annal. d. Chem. und Pharm. cxcii. 169, 1879.

2 Compare Hesse, Annal. d. Chem. und Pharm. cxcii. 173, 1878.

3 Jungfleisch and Lefranc, Comptes rendus, xciii. 547.

4 Journ. d. Pharm. et de Chim. xxii. 47, 1875. The name of chylariose has been given by Mauméné to the lævo-rotatory constituent of invert-sugar. 
According to the researches of Scheibler, ${ }^{1}$ a freshly prepared solution of arabinose possesses a rotatory power $(\alpha)_{\mathrm{D}}=+121^{\circ}$, diminishing, after heating, to $+116^{\circ}$. It crystallizes, far more easily than dextrose, in rhombic crystals ; but is not directly fermentable. Its action on Fehling's solution resembles that of grape-sugar. As already observed Kiliani maintains that arabinose, when treated with nitric acid, yields mucic acid, and is identical with lactose. (This is contradicted by Clæsson.)

For caragheen-sugar see Benté. ${ }^{2}$ It is said to be optically inactive, and to reduce Fehling.

Galactose, regarded by Fudakowski ${ }^{3}$ as a mixture of lactose and another glucose, also belongs to this group. It differs from most other glucoses in yielding mucic instead of oxalic acid when treated with nitric acid. (See also $\$ 195$.)

$\S 206$. Sorbin, Inosite, etc.-Sorbin, inosite, and eucalyn differ considerably in their properties from the foregoing sugars, but correspond to the glucoses in formula.

Sorbin can be obtained in colourless crystals, which are freely soluble in water, sparingly in cold spirit. It reduces Fehling, possesses a rotatory power $(\alpha) j=-46.9^{\circ}$; but is not fermentable. Sorbin must not be confounded with the sorbite belonging to the mannite group (\$212).

Inosite, or phaseomannite, forms colourless rhombic plates melting at $210^{\circ}$, soluble at $19^{\circ}$ in 6 parts of water, insoluble in cold absolute alcohol and ether. It is not fermentable, does not reduce Fehling, and is optically inactive. ${ }^{4}$ When warmed with nitric acid and dried, inosite is said to leave a residue that is coloured purple or blue by ammonia and chloride of calcium.

Eucalyn has been obtained, up to the present time, only in the form of an uncrystallizable, non-fermentable syrup. It possesses for $(\alpha)_{r}$ a dextro-rotatory power of $65^{\circ} .^{5}$

1 Ber. d. d. chem. Ges. vi. 612, 1873.

2 Ibid. ix. 1157, 1876.

3 Ibid. viii. 599,1875 ; ix. $42,1876$.

${ }^{4}$ On the occurrence of inosite in the vegetable kingdon (grape-juice) see Hilger, Annal. d. Chem. und Pharm. clx. 333, 1871 (Year-book Pharm. 156, 1872) ; also Neugebauer, Zeitschr. f. anal. Chem. xii. 39, 1873. Neugebauer has found inosite in vine-leaves also, and at the same time discusses the detection of quercetin, quercitrin, etc. Nucite, which has been discovered in hazelleaves, appears to be very similar to inosite (Jahresb. f. Pharm. 167, 1877).

B For some other saccharine bodies that have been hitherto but little investigated, such as dambose, etc., see Ber. d. d. chem. Ges. vi. 1314, 1873. 
\$ 207. Saccharoses.-Cane-sugar differs from the glucoses in composition; its formula, $\mathrm{C}_{12} \mathrm{H}_{22} \mathrm{O}_{11}$, is the same as that of certain carbohydrates of the vegetable gum group, triticin, etc. It crystallizes with tolerable facility in anhydrous monoclinic crystals, is freely soluble in cold water, insoluble in ether, and in cold absolute alcohol; the latter, when heated to boiling, dissolves about 1.25 per cent. An aqueous solution is dextro-rotatory. Hesse ${ }^{1}$ found the rotatory power for $(\alpha)_{\mathrm{D}}$ to be $+67.95^{\circ}(p=1)$ and $+66.50^{\circ}$. $(p=10) ;{ }^{2}$ Tollens, $^{3}+66.649^{\circ}$, and $+66.475^{\circ}(p=10)$; and Schmitz, $+66.527^{\circ}$. Heated for some time to about $160^{\circ}$, cane-sugar is converted into dextrose and levulosan $\left(\mathrm{C}_{6} \mathrm{H}_{10} \mathrm{O}_{5}\right)$; at a higher temperature it is decomposed into caramel and other products. It does not reduce alkaline copper solution in the cold; prolonged boiling, however, causes the gradual separation of cuprous oxide. Cane-sugar is only indirectly fermentable, being first graaually converted into glucose by the invertin of the yeast ( $\$ 230)$. It has already been observed ( $\$ 86,88$, etc.) that a similar change is easily brought about by the action of dilute acids; the principal means of distinguishing cane-sugar from the more important glucoses have also been mentioned (see $\$ \S 200$ to 204 and $\S 206$ ).

The following saccharoses are isomeric with cane-sugar and classed with it.

Milk-sugar, which has not yet been detected with certainty in the vegetable kingdom, forms rhombic crystals containing 1 molecule of water of crystallization and soluble in 7 parts of water at $10^{\circ}$. Its rotatory power (in solutions that have been previously heated) is $(\alpha)_{\mathrm{D}}=+53.63^{\circ}(p=2)$; Hesse found for freshly prepared cold solutions $(\alpha)_{\mathrm{D}}=+80.68^{\circ}$. It is not directly fermentable, but, like cane-sugar, is gradually inverted by yeast. The action of dilute acids resulis in the production of galactose $(\S 205)$; by oxidation with nitric acid it yields large quantities of mucic acid. Milk-sugar reduces ammoniacal silver and alkaline copper solution in the cold, but the extent to which reduction is effected when boiled with the latter reagent is less than is the case with dextrose. When converted into galactose the reducing power

${ }^{1}$ Loc. cit. Calderon found $(\alpha)_{D}=67 \cdot 09^{\circ}$ (Journ. de Pharm. et de. Chim. xxiv. 437,1876$)$.

2 For the decrease in rotatory power caused by the presence of certain salts see Muntz, Ber. d. d. chem. Ges. ix. 962, 1876.

${ }^{3}$ Ber. d. d. chem. Ges. x. 1403,1877 ; xi. 1800 , 1878. See also Schmitz, ibid. x. 1414. 
is about the same as that of invert-sugar, so that after inversion 10 cc. of Fehling indicate about 0.0475 gram milk-sugar. ${ }^{1}$

To Maltose, produced together with dextrin by the action of diastase on starch, Schulze ${ }^{2}$ assigns the formula $\mathrm{C}_{12} \mathrm{H}_{22} \mathrm{O}_{11}+\mathrm{H}_{2} \mathrm{O}$. It resembles milk-sugar in its behaviour to alkaline copper solution. Boiling with dilute acids converts it entirely into dextrose, and, as with milk-sugar, it is advisable to invert maltose before estimating it with Fehling's solution. Its rotatory power is greater than than that of dextrose $;(\alpha)_{D}=149.5-150 \cdot 6^{\circ}$. Maltose is said to be (? directly) fermentable. With nitric acid it yields no mucic acid. Melitose crystallizes in needles containing 3 molecules of water. It is soluble in 9 parts of cold water, and is dextro-rotatory $\left(+102^{\circ}\right)$. Boiling with dilute acids converts it into glucose and eucalyn, the latter of which is not fermentable. Yeast also acts in a similar manner ( $\$ 206)$, in which case the glucose produced ferments. With nitric acid melitose yields abundance of mucic acid. Melezitose crystallizes in rhombic prisms with 1 molecule of water of crystallization. It is freely soluble in water, insoluble in alcohol and in ether. An aqueous solution is dextro-rotatory $\left(94 \cdot 48^{\circ}\right)$, and indifferent to Fehling's solution ; it is converted into glucose when boiled with a dilute acid; yields with nitric acid no mucic acid, and is slowly decomposed by yeast.

Mycose (trehalose) forms rhombic prisms containing 2 molecules of water. Boiling spirit dissolves it tolerably freely; in aqueous solution it is powerfully dextro-rotatory $\left(+220^{\circ}\right)$, only slowly and incompletely fermented by yeast, and converted into dextrose when boiled with dilute acid for several hours. It does not reduce copper solution, and yields no mucic acid with nitric acid.

§ 208. Estimation by Polarization.-This is possible when the glucose or saccharose is not accompanied by any other similar carbohydrate, and when the solution contains no other substance (asparagine, etc.) that has an action on polarized light, or when all such substances present can be completely removed, either by boiling (as albumen), or by precipitation with basic acetate of lead (organic acids, gum, etc.) (\$210). Of course the rotation-

1 Compare Rodewald and Tollens, Ber. d." d. chem. Ges. xi. 2076, 1878 (Year-book Pharm. 77, 1879).

2 Ber. d. d. chem. Ges. vii. 1047, 1874 (Year-book Pharm. 85, 1875), and Journ. f. Landwirthschaft, xxvi. 67, 1878. See also O'Sullivan, Moniteur scient. March, 1874; and Ber. d. d. chem. Ges. ix. 281, 1876 (Journ. Chem. Soc.). 
constant of the carbohydrate in question must be known. The results are generally calculated from the equation

$$
\mathrm{C}=\mathrm{A} \frac{a}{\mathrm{~L}}
$$

where $\mathrm{C}$ is the weight of the substance (expressed in grams) contained in a litre of solution, A the rotation-constant of the substance to be estimated, $a$ the observed rotation, and $\mathrm{L}$ the length of the column of solution in millimetres. If the concentration of the solution has an influence on the optical activity, then $p$, the weight of substance in grams in $100 \mathrm{cc}$. of the solution, must also be taken into account. ${ }^{1}$ The same must be done for the temperature when that exerts any influence on the rotatory power (invert-sugar) (cf. § 199).

Suppose for instance a solution of grape-sugar in a tube 0.1 metre long to have shown a rotatory power $+3^{\circ}$, then (using Tollens and Grote's constant),

$$
\mathrm{C}=1883 \cdot 2 \times \frac{3}{100}
$$

For a solution of levulose with rotatory power $-3^{\circ}$,

$$
\mathrm{C}=943.4 \times \frac{3}{100}
$$

For a solution of cane-sugar with rotatory power $+3^{\circ}$ (using Wild's constant),

$$
\mathrm{C}=1505.6 \times \frac{3}{100}
$$

In the first instance the solution would contain 56.496 grams of dextrose per litre ; in the second, 28.302 grams of levulose in the third, $45 \cdot 168$ grams of cane-sugar. See also $\S 210$.

$\S 209$. Estimation of two Sugars.-Two sugars present in solution together may be accurately estimated, provided that the rotatory power of each is known, and the other conditions in $\S 208$ are fulfilled. This is frequently the case with solutions of dextrose and levulose. Neubauer ${ }^{2}$ then recommends observing the rotatory power, and estimating the sugar with Fehling's solution. If the latter indicates 15 per cent. of sugar, then if levulose alone were present, the rotatory power (by sodium-light, in a tube 0.1 metre

${ }^{1}$ See Hesse, Annal. d. Chem. und Pharm. clxxvi. 95, 1875, and Tollens, Ber. d. d. chem. Ges, xi. 1800.

${ }^{2}$ Ber. d. d. chem. Ges. x. 827, 1877. 
long) should be $-15^{\circ}(=15 \times 1 \cdot 0),{ }^{1}$ and for dextrose alone $7 \cdot 96^{\circ}$. Supposing now the angle of rotation observed to have been $-5.202^{\circ}$, that is $-9.798^{\circ}$ less than levulose alone, the amount of the latter present can be calculated from the statement

$$
2883 \cdot 3: 1883 \cdot 3:: 9 \cdot 798: x
$$

where 2883.3 is the difference between the rotation-constants of grape- and fruit-sugar $\left\{1883 \cdot 3-\left(-1000^{\circ}\right)\right\}$, and $1883 \cdot 3$ the constant of grape-sugar; multiplying out

$$
\begin{aligned}
x & =0.65317 \times 9.798 \\
& =6.4
\end{aligned}
$$

which is the percentage of levulose, leaving $15 \cdot 0-6 \cdot 4=8 \cdot 6$ per cent. of dextrose.

§ 210. Cane- and Invert-sugar.-A similar course may be pursued in other cases involving the simultaneous estimation of two sugars. For cane- and invert-sugar, as they occur for instance in beet-juice, it is usual to determine the rotatory power both before and after inversion with a dilute acid. If cane-sugar is present, more invertsugar will be found than would correspond to the rotatory power first observed.

According to Haughton, ${ }^{2}$ the rotatory power of mixtures of caneand invert-sugar cannot be correctly determined unless the excess of the basic acetate of lead, used to clarify the solution, is first removed. He thinks that the lead forms a dextro-rotatory compound with levulose. Such solutions containing lead are also said to yield inaccurate results with Fehling's solution.

If the use of basic acetate of lead alone is insufficient to render such liquids as beet-juice, etc., clear and colourless enough for examination in the polariscope, it may sometimes be advantageously combined with solution of alum. It is frequently undesirable to decolourize with charcoal, as that is capable of retaining some of the sugar.

The presence of asparagine can be the source of considerable error : firstly, because that substance is itself optically active; and secondly, because its-rotatory power varies as the solution is acid or alkaline. Moreover, it is resolved, by boiling with hydrochloric

${ }^{1}$ Calculated from equation $a=\frac{10}{10 \cdot 0}$. Neubauer takes the rotatory power of levulose to be $-100^{\circ}$ instead of $-106^{\circ}$.

2 Journ. Chem. Soc. ix, 85 ; Zeitschr, f. anal. Chem. x. 490, 1871. 
acid, into aspartic acid and ammonia; the former of which is lævo-rotatory in alkaline solution, but dextro-rotatory in acid.

Asparagine is not precipitated by basic acetate of lead, by lime, or by baryta; and although aspartic acid is thrown down by lead salts, yet the precipitate is redissolved by an excess. ${ }^{1}$

§ 211. Estimation of three Sugars.-Dupré ${ }^{2}$ and Apjohn ${ }^{3}$ have made the estimation of cane-, grape-, and fruit-sugar in the same solution, the subject of investigation. As their methods, however, involve a combination of optical and volumetric determinations in which accuracy is required in ascertaining the amount of grape- and fruit-sugar present, especially in titrating, I am afraid their processes will at present yield only approximate results. I have already shown ( $(85)$ that the volumetric estimation of glucose in the presence of cane-sugar is not so accurate as might be wished.

§212. Mannite.-Of mannite (cf. $\S 91$ ) it may be here observed that one part dissolves in $6 \cdot 14$ to $6 \cdot 21$ of water at $15^{\circ}$, and in $5 \cdot 12$ to 5.38 at $20^{\circ}$. One part requires also 1515 of absolute alcohol at $17^{\circ}$ for complete solution. Although optically inactive, as previously stated, it is dextro-rotatory when dissolved in a concentrated solution of borax. It melts at $166^{\circ}$, and is converted at $200^{\circ}$ with loss of water into mannitan. With moderately strong nitric acid it yields principally mucic and saccharic acids; with the strongest acid, nitro-mannite. It is said (according to Riegel) to reduce gold and silver salts, but not alkaline copper solution, when warmed for a short time. Mannite prevents, however, the precipitation of hydrate of copper.

${ }^{1}$ On saccharometry, and especially the estimation of invert-sugar when accompanied by cane-sugar, as in beet-juice, crude sugar, etc., see Ventzke, Journ. f. pract. Chem. xxv. 65, xxviii. 101; Kleinschmidt, Dingler's polyt. Journ. clxxxi. 306, 1867 ; Anders, ibid. clxxxii. 331 ; Bodenbender, Zeitschr. f. Chem. N. F. ii. 222, 1867 ; Sostmann, ibid. 480. The latter authors draw special attention to the infuence that lime exercises on the results of optical determinations of cane-sugar. The influence of asparagine and aspartic acid has been observed by Dubrunfaut (Dingler's polyt. Journ. cxxi. 305) and Scheibler, ibid. clxxxi. 415). Communications by Scheibler on the errors in optical estimations have appeared in the Zeitschr. f. Chem. N. F. iii. 617, and Zeitschr. f. anal. Chem. viii. 211, 1869. See also Stammer, Dingler's polyt. Journ. clxxxii. 160; Dubrunfaut, ibid. cxxi. 299, clxxxv. 231; Landolt, Zeitschr. f. anal. Chem. vii. 1, 1868. On the influence of asparagine, see lastly also Champion and Pellet, ibid. xvi. 120, 1877.

2 Chem. News, xxi. 97, 1870 .

${ }^{3}$ Ibid. p. 86. See also Zeitschr. f. anal. Chem. ix. 499, 501, 1870. 
Dulcite, or melampyrite, is isomeric with mannite, and shares most of its important properties. The crystals of the former belong to the monoclinic system, those of the latter to the rhombic. Dulcite dissolves in three parts of cold water, is optically inactive, does not ferment, and melts at $182^{\circ}$.

Isodulcite (rhamnodulcite) is also isomeric with the foregoing. Crystals of isodulcite melt at $93^{\circ}$ to $94^{\circ}$, dissolve in 2.09 parts of water at $18^{\circ}$, and also in absolute alcohol. It is not fermentable, but reduces Fehling's solution, and is dextro-rotatory, $(\alpha) j=8 \cdot 4^{\circ}$.

Hesperidin-sugar and sorbite are also said to be isomeric with mannite. The former crystallizes in the monoclinic system, is dextro-rotatory, sparingly soluble in hot absolute alcohol, but more easily in hot 70 per cent. spirit. It melts at $70.5^{\circ}$ to $76^{\circ}$, and slowly reduces Fehling. Anhydrous sorbite melts at about $110^{\circ}$, and does not reduce Fehling. ${ }^{1}$

$\S 213$. Quercite, etc. -Quercite and pinite are isomeric with mannitan and dulcitan and are sweet to the taste.

Quercite crystallizes in the monoclinic system, dissolves in 8 to 10 parts of cold water, is dextro-rotatory $\left(+33.5^{\circ}\right)$, and melts at $235^{\circ}$.

Pinite is indistinctly crystalline, freely soluble in cold water, and somewhat soluble in dilute spirit; it melts at $150^{\circ}$, and has a dextro-rotatory power of $58.6^{\circ}$.

Abietite differs from the foregoing in containing the elements of a molecule of water less ; it has been the subject of communications by Rochleder. ${ }^{2}$

\section{ACIDS.}

$\$ 214$. Detection of Acids. - In addition to the reaction of malic ucid mentioned in $\S 81$, Barfoed ${ }^{3}$ describes another depending upon the conversion of the acid, at a temperature of $160^{\circ}$ to $170^{\circ}$, into maleïc and fumaric acids, with production of a crystalline sublimate. A third is the lime-reaction; malate of lime requires the addition of at least 1 to 2 volumes of spirit for precipitation; and when thus

${ }^{1}$ Compare Boussingault, Journ. de Pharm, et de Chim. xvi. 36, 1872 (Pharm. Journ. Trans. [3], iii. 28).

${ }^{2}$ Journ. f. pract. Chem. cv. 63, and Apoth.-Ver. viii. 363, 1868. For catharto-mannite, see Kubli and Dragendorff, Pharm. Zeitschr. f. Russland, iv. 467, 1865, and Keussler, ibid. xvii. 363, 1878. For bergenite, see Morelle, Chem. Centralblatt, 758, 1881 (Comptes rendus, xciii. 646).

${ }^{3}$ Zeitschr. f. anal. Chem. vii. 403, 1868. 
thrown out of solution, softens on warming and aggregates to a mass, which becomes granular and crystalline on cooling. The magnesia-reaction, mentioned by the same author as a new one, consists in converting into malate of magnesia either by saturating the acid with oxide or carbonate of magnesia, or by mixing a concentrated solution of an alkaline malate with chloride of magnesium. The salt is precipitated by spirit from a hot solution, as a tenacious mass hardening on cooling. (Citrate of magnesia behaves in a similar manner.)

Barfoed separates malic from oxalic (\$ 219) and tartaric (\$217) acids by precipitating the last two from neutral solution by chloride of calcium, and throwing out malate of calcium from the filtrate by the addition of alcohol. If tartaric acid is present, it must be remembered that the calcium-salt of this acid separates but slowly. (See also. §218.)

In separating citric (\$215) from malic acid the same chemist takes advantage of the fact that citrate of calcium requires less spirit for precipitation than malate.

If malic acid is in solution with oxalic, tartaric, and citric acid, Barfoed advises the precipitation of the last three as neutral ammonium salts by the addition of 7 to 8 volumes of 98 per cent. spirit; after standing twelve to twenty-four hours the precipitate is filtered off, and the malic acid thrown down in the filtrate with acetate of lead.

Succinic acid (\$220) can be separated from malic acid, according to Barfoed, by converting into neutral alkaline salts, precipitating with acetate of lead, dissolving the precipitate in acetate of ammonia solution, and then separating the malate of lead by the addition of 2 volumes of spirit; or the concentrated aqueous solutions of the neutral potassium, or sodium-salts, may be mixed with about 6 volumes of spirit, when the succinate remains in solution.

For the separation of malic acid from gallotannic (\$\$ 49 et seq. and 165), gallic (§151), benzoic (§26), acetic and formic (§139), acid, reference must be made to the original work.

To remove sulphuric and phosphoric acids from solutions in which malic acid is to be detected, Barfoed recommends separating the first two by the addition of chloride of barium to the hot solution, and precipitating the malate of barium from the filtrate by the addition of alcohol.

§215. Estimation of Citric Acid.-In estimating citric acid, Creuse 
takes advantage of the insolubility of the barium-salt in spirit. To a neutral solution of the citrate a solution of acetate of barium and 2 volumes of spirit are added. The precipitate is filtered off, ignited, converted into sulphate by sulphuric acid, and weighed as such. The composition of citrate of barium is said to be $\mathrm{Ba}_{3} \mathrm{C}_{12} \mathrm{H}_{10} \mathrm{O}_{14}$. (Compare Zeitschr. f. anal. Chem. xi. 446, 1871.) $)^{1}$ Precipitation by barium had been previously recommended by Kämmerer as a means of detecting citric acid. ${ }^{2}$ If to a solution of a citrate excess of acetate of barium is added, a voluminous precipitate is obtained, which gradually becomes crystalline (prisms) when heated for several hours on the water-bath. The presence of other fruit-acids does not interfere. If the solution is very dilute it must be concentrated after the addition of the barium salt, as otherwise only acicular crystals are produced. See further $\$ \$ 218$ to 220 .

\$216. Qualitative Reactions of Citric Acid.-Sarandinaki has drawn attention to the fact that the triethylic ether of citric acid decomposes when heated in sealed tubes to $110^{\circ}$, with separation of a blue powder $;^{3}$ and that an aqueous solution of citrate of ammonium, similarly treated for six hours, also deposits a blue decomposition-product on subsequent exposure to air and light in a flat dish. Sabanin and Laskowsky ${ }^{4}$ have shown that neither tartaric, malic, nor oxalic acid interferes with the reaction which succeeds with 0.01 gram of citric acid. Aconitic acid comports itself like citric. If the juice of a fruit or a vegetable infusion is under examination, the citric acid can be precipitated as citrate of lead, the latter converted into citrate of barium, and finally into citrate of ammonium, which can be tested as above described.

It may also be observed that an aqueous solution of citric acid is optically inactive. According to Roennefahrt, 100 parts of ether dissolve 10 parts of the crystallized and 13 of the anhydrous acid, and can remove as much as 3.6 parts when shaken with an aqueous solution; facts scarcely in accordance with the statement

1 For quantitative estimation of tartaric acid, see also Inette, Comptes rendus, lxvi. $417,1868$.

${ }^{2}$ Annal. d. Chem. und Pharm. cxlviii. 294, and Zeitschr. f. anal. Chem. viii. $298,1869$.

${ }^{3}$ Ber. d. d. chem. Ges. 1100, 1872. Compare also Kämmerer, ibid. 736, 1875 (Journ. Chem. Soc. xxix. 496, xxviii. 1178).

4 Zeitschr. f. anal. Chem. xvii. 73, 1878 (Year-book Pharm. 165, 1878). 
formerly made to the effect that citric acid was difficultly soluble in ether.

§217. Precipitation of Tartaric Acid (\$ 82).-As early as 1864, Berthelot and de Fleurieu precipitated tartaric acid by converting it into acid tartrate, and adding 5 volumes of ether-alcohol. ${ }^{1}$ Jokisch and Bolley ${ }^{2}$ have shown that acid tartrate of calcium may be simultaneously thrown down.

For the estimation of the total acid in must, see Pasteur. ${ }^{3}$

Martenson ${ }^{4}$ precipitates tartaric acid from a 1 per cent. aqueous solution of normal tartrate of potassium by the addition of chloride of calcium and lime-water. The sides of the porcelain dish in which the operation is performed should not be touched by the stirring-rod. On standing for several hours, the calcium-salt separates in crystals, and can be filtered off, washed with 80 to 85 per cent. spirit, dried at $100^{\circ}$ and weighed; its composition is then $\mathrm{CaC}_{4} \mathrm{H}_{4} \mathrm{O}_{6}, 4 \mathrm{H}_{2} \mathrm{O}$.

$\S 218$. Estimation of Tartaric and Citric Acid.-Fleischer recommends the separation of the former as acid tartrate of potassium, the precipitation being rendered complete by the addition to the solution of twice its volume of 95 per cent. spirit. The acid tartrate is filtered off, redissolved, and estimated by titration with normal alkali (\$82).

The citric acid is precipitated from the filtrate by neutral acetate of lead, washed with spirit of about 45 to 50 per cent., liberated by sulphuretted hydrogen, and estimated by titration with normal alkali.

This method is directly applicable if the acids are present in the free state, or in combination with alkalies. ${ }^{5}$

${ }^{1}$ Comptes rendus, lxxvii. 394.

2 Dingler's polyt. Journ. clxxxiii. 47, 1867 (Amer. Journ. Pharm. xxxvi. 60). See also Kissel, Zeitschr. f. anal. Chem. viii. 409, 1869.

${ }^{3}$ Zeitschr. f. anal. Chem. viii. 86, 1869.

${ }^{4}$ Pharm. Zeitschr. f. Russland, viii. 23, 1868 (Amer. Journ. Pharm. xli. 335).

5 See also Schnitzer, Dingler's polyt. Journ. clxiv. 132, 1862, on the separation of tartaric from citric acid as acid tartrate.

Should it appear desirable to estimate the citric acid directly, after removal of the tartaric as acid tartrate of potassium, the filtrate may be mixed with chloride of calcium and concentrated by boiling. The citrate of calcium that separates after adding lime-water should be filtered off hot, washed with boiling water, dried and weighed (258 parts of the dried salt indicate 192 of citric acid). See also Allen, Zeitschr. f. anal. Chem. xvi. 251, 1877.

For distinctive characters of tartaric and citric acid, see Archiv d. Pharm. clviii. 206, 1861; Chapman and Smith, Laboratory, April, 1868; Wimmel, Zeitschr. f. anal. Chem. vii. 411, 1868. 
If the solution contains oxalic and sulphuric, in addition to tartaric and citric acids, all of them must be precipitated with acetate of lead (which would also throw down part of any chlorine that may be present); the precipitate should be washed with dilute spirit and treated with ammonia (free from carbonate), which dissolves citrate and tartrate of lead and part of the chloride. The tartaric acid may be estimated in the solution by decomposing the lead-salts with sulphide of ammonium and acetic acid, filtering, and precipitating with acetate of potassium as above described. If chloride is present, the citric acid must be subsequently-precipitated from a hot solution, either as lead-salt by excess of acetate (washing with dilute spirit) or as calcium-salt in the presence of spirit; the latter must be redissolved in acetic acid, and precipitated with acetate of lead.

If lime and phosphates are also present (in hydrochloric acid solution), they may be precipitated by acetate of ammonia as oxalate of calcium and phosphate of iron, and washed with solution of chloride of ammonium to avoid loss of tartaric acid as acid tartrate of calcium. The phosphoric acid may be subsequently precipitated as phosphate of lead, which is insoluble in ammonia. The residue of the lead precipitate, after treatment with ammonia, can be decomposed by sulphide of ammonium, and in the filtrate from the sulphide of lead, phosphoric, sulphuric, and oxalic acids determined by the usual processes.

Malic acid if present would be precipitated together with the citric acid as calcium salt; the former, however, is soluble in boiling lime-water.

Racemic acid would be thrown down with the tartaric acid, but could be separated by dissolving the acid tartrate and racemate in hydrochloric acid, supersaturating with ammonia and adding chloride of calcium, which, under these conditions, precipitates racemate of calcium only. ${ }^{1}$

Racemic acid is optically inactive, whilst tartaric acid is dextrorotatory $(\alpha)_{\mathbf{D}}=+14 \cdot 18^{\circ}(p=5)$, or $(\alpha)_{\mathbf{D}}=+12 \cdot 80^{\circ}(p=15)$. It is well-known, however, that racemic acid can be made to yield lævoracemic acid by suitable treatment.

For detection of tartaric acid in presence of malic, formic acids, etc., see Braun, Zeitschr. f. anal. Chem. vii. 349, 1868; Kämmerer, viii. 300, 1869. See also Cailletet, xvii. 499, 1878 (Year-book Pharm. 150, 1878).

${ }^{1}$ Compare Fleischer, Archiv d. Pharm. [3], v. 97, 1874 (Journ. Chem. Soc. xxvii. 1181). 
Both tartaric and racemic acids are easily soluble in water and alcohol. See also $\$$ 219, 220.

§219. Separation of Oxalic Acid.-Of the acids above referred to, oxalic acid (\$\$ 81, 214, 216, 218) splits up, when heated, into water, carbonic acid, and carbonic oxide without separation of carbon. It undergoes a similar decomposition when warmed with concentrated sulphuric acid. In addition to the reactions already described, it must be observed that a neutral solution of an oxalate throws down bright yellow crystalline ferrous oxalate, when warmed or allowed to stand, with a solution of a ferrous salt. According to Barfoed, ${ }^{1}$ the precipitate is soluble in about 4,000 parts of cold water, but tolerably easily in solutions of oxalates, tartrates, and citrates of the alkalies. The reagent must therefore be added in sufficient quantity to convert the whole of the vegetable acids present into salts of iron.

Tartaric acid, if present, might also yield a crystalline precipitate, but the colour would be dirty-white and the crystals would be distinguishable under the microscope; it might be separated by cold, very dilute hydrochloric acid, in which the oxalate is almost insoluble. Citric acid delays the precipitation, and frequently prevents the formation of characteristic crystals, which, however, may be obtained by dissolving in a little warm hydrochloric acid and neutralizing with ammonia.

Oxalic acid can be isolated from oxalate of calcium (\$110) for the purposes of further identification by dissolving in hydrochloric acid, and adding sufficient sulphuric acid to convert into sulphate, which can then be completely precipitated by the addition of an equal volume of spirit. Or the oxalate may be dissolved in the smallest possible quantity of dilute nitric acid, precipitated with nitrate of lead, and liberated from the lead-salt by sulphuretted hydrogen. (Cf. $§ 81,218,220$, where the estimation of oxalic acid is discussed.) Oxalic acid requires 10 parts of cold water for solution, but only 2 parts of alcohol.

$\S 220$. Succinic Acid ( $\$ 214)$ is also one of those organic compounds that are not blackened by concentrated sulphuric acid, and leave no carbonaceous residue when heated on platinum foil, or in a test-tube. In the latter case the major part is volatilized without decomposition. It differs from oxalic acid in being less soluble in cold water (about 1 in 15 at $20^{\circ}$ ), and in being spar-

1 'Lehrb. d. org. qual. Analyse,' Copenhagen, 1880. 
ingly dissolved by alcohol, and to but a very slight extent by ether. The occurrence of succinic acid is frequently mentioned in plant-analyses of less recent date, but in many instances confirmation of such statements is required. And even if that were forthcoming, the question might be raised as to whether the acid had not been produced by a process of fermentation that might have taken place during the manipulation.

The following reactions may serve for the identification of succinic acid :

- Chloride of barium precipitates from a solution of an alkaline succinate (in presence of alcohol) a crystalline barium-salt, which is soluble in about 250 parts of water, but almost insoluble in alcohol. Hydrochloric acid dissolves it, but acetic acid takes up only small quantities at the ordinary temperature. ${ }^{1}$

Chloride of calcium also throws down a crystalline salt from concentrated solutions of alkaline succinates. The precipitate dissolves, according to Barfoed, in about 50 parts of water, but is very sparingly soluble in alcohol. If the precipitation takes place in the presence of alcohol the salt is at first amorphous, and subsequently assumes a crystalline condition. It dissolves in warm dilute acetic acid, and in boiling solution of chloride of ammonium. (Distinction from oxalic acid.)

Succinate of lead is likewise crystalline, but complete separation takes place very slowly. Basic acetate of lead throws down an amorphous salt. Ferric chloride produces brownish-red amorphous precipitates in solutions of normal alkaline succinates, but does not form insoluble compounds with oxalates.

In separating oxalic from succinic acid advantage may be taken of the solubility of ammonium succinate in spirit. Barfoed separates tartaric from succinic acid in the form of acid tartrate of potassium in the presence of hydrochloric acid and alcohol. $\mathrm{Or}$ it may be precipitated by mixing the hot solution with chloride of ammonium, adding chloride of calcium and cooling. Citric acid may be separated in a similar manner, but in this case the mixture must be boiled until the whole of the citrate of calcium has been thrown down; or the solution may be boiled with chloride of barium and filtered whilst hot from the precipitated citrate of barium. The filtrate contains the succinate of barium, which can be thrown down by alcohol after cooling (\$215).

1 See Barfoed, loc. cit. 
For the separation of malic acid, see $\S 214$.

\$221. Fumaric acid can also be sublimed, and, like oxalic and succinic acids, yields a precipitate with acetate of lead that gradually assumes a crystalline condition (\$\$ 81, 214). It differs from malic acid in its slight solubility in water (according to Lassaigne about 1 in 260 at $17^{\circ}$ ). It is much more easily soluble in hot water, and is also dissolved by ether and by cold spirit. The solubility of the silver-salt is extremely slight; manganous fumarate is also sparingly soluble in water, but freely in spirit. The acid potassium and acid ammonium salts are not very soluble in water, and scarcely at all in cold 80 per cent. spirit. Lime-water does not precipitate fumaric acid.

Maleic acid is isomeric with the preceding, and, like it, is optically inactive and yields a crystalline lead-salt. It is freely soluble in water, but cannot be sublimed without decomposition.

Kinic acid may also be mentioned here. It is precipitated by ammoniacal, but not by normal acetate of lead; and when saturated with milk of lime, yields at first a calcium-salt soluble in water.

Many other vegetable acids can be separated from kinic acid by precipitation with normal acetate of lead; the filtrate, after removal of the excess of lead as sulphide, can be freed from sulphuretted hydrogen, boiled with milk of lime, concentrated, and allowed to stand, when a crystalline deposit of kinate of calcium will gradually separate, or more rapidly if spirit is added. The presence of kinic acid in the deposit may be demonstrated by heating with sulphuric acid and black oxide of manganese; the quinone thus produced is volatile, crystallizes in yellow needles, and can be detected even in traces by its iodine-like odour ( $\$ 167$, arbutin).

Kinic acid and its silver-salt are easily soluble in water. ${ }^{1}$

\$222. Lactic Acid.-Although the occurrence of lactic acid in living plants has not been placed beyond doubt, we must devote a few lines to it here, as it is frequently produced from carbohydrates when vegetable infusions are allowed to stand, and is therefore often met with in plant-analysis. It can be detected by

1 For rubichloric acid, which occasionally accompanies kinic acid and, like it, is only precipitated by alkaline acetate of lead solution, see Schwarz, Sitzungsber. d. Akad. d. W. in Wien Math. nat. Cl. 26, 1852. Rubichloric acid is decomposed with simultaneous separation of green insoluble chlorrubin when its aqueous solution is boiled with dilute hydrochloris acid. 
shaking the aqueous liquid with ether. On evaporating the ethereal solution, it is obtained in the form of a syrup, easily soluble in water and in alcohol. Both the calcium and lead salts are freely dissolved by water, the former requiring about 10 parts at the ordinary temperature. Lactic acid can thus be separated from many other acids by precipitation with acetate, or, if free acids are present, by digestion with carbonate of lead or calcium. Lactate of calcium is further soluble in boiling 85 per cent. spirit, and crystallizes from hot saturated spirituous and aqueous solutions on cooling.

From a spirituous solution of lactate of lime ether throws down an amorphous precipitate, which gradually becomes crystalline. ${ }^{1}$

The magnesium and zinc salts are also crystalline; the former is best prepared by precipitating a concentrated solution of lactate of soda with chloride of magnesium in the presence of alcohol; the latter is frequently of use in detecting lactic acid. Lactate of magnesium requires about 30 parts of cold water for solution, of zinc about 60 ; both are far more easily soluble in hot water. If the presence of lactic acid is suspected, the aqueous solution should be concentrated, digested hot with oxide of zinc, cooled, and examined under the microscope. It should show bundles of needles and sphærocrystalline masses, as well as four-sided prisms and wedge-shaped crystals. Paralactate (sarcolactate) of zinc is much more easily soluble, requiring only about 6 parts of cold water. Acicular crystals are also obtained if carbonate of silver is used instead of oxide of zinc; they are soluble in alcohol.

Air-dry lactate of calcium contains $29 \cdot 2$ per cent. of water and $18 \cdot 3$ per cent. of oxide of calcium.

The zinc-salt contains 18.2 per cent. of water and $27 \cdot 3$ per cent. of oxide of zinc.

\$223. Glycolic Acid.-Glycolic acid is homologous with lactic acid, and resembles it in many of its properties. It has been found in the juice of unripe grapes. Ethereal solutions yield the acid in crystals which melt at $78^{\circ}$ to $79^{\circ}$, and are partially volatile in the vapour of water without decomposition. Glycolate of barium dissolves in 7.9 parts of cold water, of calcium in 80.8 , of zinc in $31 \cdot 6$, of lead (normal salt) in $31 \cdot 17$. The last three can be obtained in crystals. The precipitate produced by basic acetate of lead is very sparingly soluble (about 1 in 10,000), and

\footnotetext{
${ }^{1}$ Compare Barfoed, loc. cit. 144.
} 
can therefore be made available for the separation of the acid; the basic lead-salt can be converted into the normal by the action of dilute nitric acid. Glycolic acid can be isolated by shaking with ether. Its calcium-salt contains 23.09 per cent. of oxide of calcium, its normal lead-salt 62.48 per cent. of oxide of lead.

\section{ALBUMINOIDS, ETC.}

$\S 224$. Estimation. - In $\$ 96$ the albuminoids have been estimated by multiplying the nitrogen found by $6 \cdot 25$. This calculation is based upon the assumption that albuminoid substances contain 16 per cent. of nitrogen. ${ }^{1}$ Attention has, however, been frequently drawn to the fact, thatmostalbuminous substances contain more than 16 per cent. of nitrogen; those of cereals, leguminous fruits, etc., can be estimated to contain at least 16.60 per cent. ; the factor 6.0 has therefore been recommended in such cases as preferable. But even then the results obtained will be too low if the material under examination contain much conglutin or gliadin ${ }^{2}$ (as, for instance, lupin seeds, almonds, brazil-nuts, wheat, etc.), as conglutin contains $18 \cdot 4$, and gliadin $(\$ 235) 18 \cdot 1$ per cent. of nitrogen. When, therefore, large quantities of those bodies are present, Ritthausen advises multiplying by $5 \cdot 5$.

$\S 225$. Legumin.-If the experiment described in $\S 93$ has shown the presence of legumin (vegetable caseïn), the maceration should be repeated in the cold (best at a temp. of $4^{\circ}$ to $5^{\circ}$ ) for about five hours, the clear liquid decanted off, and the maceration

${ }^{1}$ It was formerly assumed that they contained 15.6 per cent. of nitrogen, and the amount of the latter was therefore multiplied by 6.33 . That the nitrogen found in alkaloids, albuminoids, etc., by the method of Varrentrapp and Will is too low has already been shown by V. d. Burg (Zeitschr. f. anal. Chem. iv. 322, 1865), Nowak (ibid. xi. 324, 1871), and Seegen and Nowak (ibid. xii. 316, 1873; xiii. 460, 1874). Meusel expresses himself to the contrary (ibid. v. 197, 1866). Märcker has shown (Annal. d. Landwirthsch. xii. 619 ) that the error is not so large if the soda lime used is free from magnesia. See also Kreusler (Zeitschr. f. anal. Chem. xii. 354, 1873); Märcker (ibid 221); Märcker und Abesser (ibid. 447); Johnson (ibid. 446); Ritthausen (ibid. xiii. 240, 1874); Settegast (ibid. xvii. 501, 1878). On the estima. tion of nitrogen, see also Nowak (Zeitschr. f. anal. Chem. xii. 102, 1873); Makris (ibid. xvi. 249, 1877); Habermann (ibid. xvii. 376, 1878); Pflüger (Archiv. f. ges. Phys. xviii. 117, 1879); Hanko (Ber.d. d. chem. Ges. xii. 451) ; Schiff (Annal d. Chem. und Pharm. cxcv. 293) ; Ritthausen (Zeitschr. f. anal. Chem. xviii. 601, 1879).

${ }^{2}$ Compare Ritthausen, Die Eiweisskörper der Getreidearten, Hulsenfrïchte etc., 237, Bonn, 1872. 
repeated once more. The legumin can then be precipitated as described. ${ }^{1}$

During all these manipulations, the exclusion as far as possible of the carbonic acid in the air is most strongly to be urged. It would be liable, in most cases, to cause an error by partially or completely precipitating albuminoids allied to (perhaps identical with) legumin, such as globulin, or vegetable vitellin.

If the material has not been previously entirely freed from fixed oil (and this is not always possible with petroleum spirit) the precipitated legumin must be finally washed with absolute alcohol and with ether, to remove the oil mechanically retained by it.

\$226. Extraction with Dilute Alkali.-Ritthausen has observed that the strongly acid reaction of many freshly powdered seeds decreases on keeping. Now strongly acid liquids are less suitable for the extraction of legumin than neutral or alkaline; Weyl, ${ }^{2}$ moreover, asserts that fresh seeds contain no legumin, and ex. presses it as his opinion that this substance is produced from vitellin and myosin during the manipulations. The differences that can thus arise are extremely inconvenient. It must also be observed that pure water frequently extracts but very small quantities of legumin from vegetable substances containing that body, and that the major part is removed only by dilute alkali. This portion of the legumin (caseïn, ${ }^{3}$ glutencaseïn, conglutin, vegetable fibrin) will therefore be dissolved when the residue insoluble in water is treated with dilute $(0.1$ to 0.2 per cent.) soda or potash ( $\S 103$ to 106). If the presence of legumin is suspected it is advisable to extract at a temperature of $4^{\circ}$ to $6^{\circ}$. (See also §233.)

Should metarabic acid ( $(195)$ and legumin be extracted together, a total estimation must first be made, and the nitrogen then determined in a part of the precipitate, from which the amount of legumin present can be calculated.

A current of carbonic acid might be passed through the alkaline extract to ascertain whether globulin (vitellin, myosin) can be thus precipitated ( $\$ 93)$; should this prove to be the case, it might be

${ }^{1}$ Compare Ritthausen, loc. cit. 144.

2 Beitr. z. Kenntniss d. thier. und pflanz. Eiweisskörper. Diss. 1877.

3 The substances here referred to agree in most of their properties, but exhibit certain differences; thus, glutencaseïn contains more sulphur, and is more easily soluble in acetic acid than legumin. Ritthausen advocates the use of the word 'casein' as a group-designation. 
rapidly filtered off with the help of a filter-pump, washed with 40 to 50 per cent. spirit, finally with absolute alcohol and with ether, dried and weighed.

$\S 227$. Isolation of Vitellin, etc. - Grains of vitellin ${ }^{1}$ (aleurone, etc.) may be frequently isolated from substances containing them in large quantities, by a process of elutriation with olive oil, ${ }^{2}$ or a mixture of oil and petroleum spirit. ${ }^{3}$ Freed from oil with petroleum spirit, vitellin dissolves in water at $30^{\circ}$ to $40^{\circ}$, and can be precipitated from such a solution by a current of carbonic acid. To obtain it in crystals the precipitate is digested with calcined magnesia at $35^{\circ}$, filtered whilst warm and cooled. Schmiedeberg considers these crystalloids, as well as aleurone itself, to be compounds of vitellin with alkalies or alkaline earths. The crystals are doubly refracting.

§228. Separation of Vitellin from Myosin.-According to Weyl, ${ }^{4}$ vegetable vitellin may be distinguished from vegetable myosin by requiring, in a solution containing 10 per cent. of common salt, a temperature of $75^{\circ}$ for coagulation, whilst myosin coagulates at $55^{\circ}$ to $60^{\circ}$. The latter, which has also been found in potatoes, passes into solution when the residue, after treatment with water, is macerated with 10 per cent. solution of salt. Small pieces of rock-salt suspended in such a solution (previously neutralized with carbonate of soda) cause the gradual precipitation of the myosin, which, however, can be redissolved by the addition of a little water. Myosin may be separated from vitellin by diluting the solution largely with water, precipitating both with carbonic acid, dissolving in dilute salt-solution and coagulating at the temperature stated.

$\S 229$. Estimation.-Experiments made by Liborius, ${ }^{5}$ Girgensohn, ${ }^{6}$ and Taraskewicz, ${ }^{7}$ have shown that the albumen of bloodserum, eggs, etc., can be estimated with tolerable accuracy by

${ }^{1}$ A similar substance occurs in the seeds of Pinus cembra. See Schuppe, Pharm. Zeitschr. f. Russland, 520, 18 S0.

${ }^{2}$ Compare Maschke, Chem. Centralblatt, 864, 1858, and Sachsse, ibid. 583, 1876 (Journ. Chem. Soc. xxxii. 200).

${ }^{3}$ Compare Schmiedeberg, Zeitschr. f. phys. Chem. i. 206 ; Ritthausen, Archiv f. die ges. Phys. xvi. 301 (Journ. Chem. Soc. xxxiv. 518).

${ }^{4}$ Loc. cit.

5 'Beitr. z. quant. Eiweissbest. Diss. Dorpat, 1870.

6 'Beitr. z. Albuminometrie und z. Kenntniss d. Tanninverb. d. Albuminate. Diss. Dorpat, 1872.

7 Einige Methoden z. Werthbest d. Milch. Diss. Dorpat, 1873. 
means of the tannin reagent mentioned in $\S 95$, and CramerDolmatow has found that extracts from one and the same plant yield concordant results when titrated with the same reagent. It must be left, however, for further experiments to show what vegetable albuminoids can be estimated in this way.

$\$ 230$. Estimation continued.-A gravimetric estimation with tannin will generally yield higher results than can be obtained by coagulation (\$94). The source of the difference is to be looked for partly in the deficiencies of the latter method, and partly in the fact that a number of albuminous substances soluble in water are not coagulated by boiling with dilute acetic acid, but are nevertheless precipitated by tannin. For this reason the results obtained by the tannin-method will generally agree better with those yielded by precipitation with alcohol. Nevertheless, I do not recommend the omission of the estimation by coagulation, for if the difference is considerable, that is, if the estimation by the tannin-method yields much higher results than that by coagulation, it proves that another albuminous substance is present, which is not coagulated by boiling. It is only when the difference is small that the presence of vegetable albumen alone may be inferred; it may then be estimated by precipitation by tannin.

To render the coagulation-test as reliable as possible, I have recommended chloride of sodium to be added, and the precipitate to be washed, first with boiling water, and subsequently with dilute spirit. If the chloride of sodium is omitted the precipitation is generally less complete, and prolonged washing, especially with cold water, is liable to redissolve part of the albumen.

Ferments. - Simultaneously with the albumen a number of other substances may be partially or wholly precipitated, which, although agreeing with albumen in many respects, have been too little investigated from a chemical point of view to justify their being classed straightway as albuminoids. I refer to the so-called ferments. Like albumen, they contain nitrogen, and are precipitated by strong alcohol, etc.; most of them, probably, are coagulated like, or together with, albumen when boiled in aqueous solution. They are distinguished from albumen by their fermentative action, which evinces itself in various ways. Diastase, like saliva, converts starch into sugar, whilst invertin changes saccharose into invertsugar. Vegetable ferments allied to pepsin (papayotin) peptonize albumen. Myrosin decomposes myronic acid, emulsin amygdalin; 
but emulsin does not attack myronic acid, nor does invertin convert starch into maltose and dextrin, etc. It is easy, therefore, to detect diastase in malt, invertin in yeast, emulsin in almonds, etc., the presence of which is anticipated. The liquefaction of starch-paste, the conversion of cane-sugar into invert-sugar, the development of hydrocyanic acid and oil of bitter almonds, are changes so striking and so promptly effected, that the qualitative detection of the ferments producing them leaves nothing to be desired. But the varied nature of the ferments themselves and of their action renders it exceedingly difficult to detect them in vegetable substances that have not previously been examined, as a general reagent applicable in such a case is yet unknown. It must be admitted that attention has been drawn to the fact that the ferments liberate oxygen from an aqueous solution of peroxide of hydrogen, to which a little tincture of guaiacum has been added, and thus produce a blue colouration of the mixture. But it is hardly to be expected that this property should be shared by all ferments, or that it should be peculiar to them alone.

$\$ 231$. Estimation of total Albumen.-The total albumen soluble in water can be estimated by means of acetate of copper, provided that no tannin or other substance precipitated by the same reagent is present in solution. The precipitate produced by an excess of the acetate is filtered off, dried, weighed, and ignited, the resulting oxide of copper being deducted. ${ }^{1}$

If other substances are thrown down with the albumen the nitrogen in the precipitate may be determined, and from that the albumen present calculated. Ritthausen ${ }^{2}$ and Taraskewic ${ }^{3}$ have proved experimentally that the precipitate contains the whole of the albumen, caseïn, etc.

$\S 232$. Estimation continued.-Sestini ${ }^{4}$ considers it advisable to precipitate with acetate of lead. In cases in which other nitro-

${ }^{1}$ In some instances it is necessary to add a considerable excess for complete precipitation of the albumen. In an experiment made by Taraskewicz with casein, 1 gram of oxide of copper (in the form of acetate) was found to precipitate 4.19 gram of casein; but for complete precipitation an amount of acetate corresponding to 4.55 grams of oxide had to be added.

${ }^{2}$ Loc. cit. 34, etc. ; Ritthausen and Settegast, Archiv f. d. ges. Phys. xvi. 293, 1877. See also Mörner, Upsala Läkarefören. Forhandl. xii. 475, 1877; Fassbender, Ber. d. d. chem. Ges. xiii. 1818, 1880 (Journ. Chem. Soc. xl. 205).

${ }^{3}$ Loc. cit.

${ }^{4}$ Landwirthsch. Versuchsst. xx. 305, 1878 (Journ. Chem. Soc. xxxiv. 740). 
genous substances accompany the albumen in aqueous solution he advises the determination first of the total nitrogen; a part of the original substance is then to be boiled with water for an hour, made distinctly acid with lactic acid, mixed with acetate of lead, and filtered; the insoluble residue is dried and the nitrogen in it estimated. He thus assumes that all the nitrogen not present in the form of albuminoids passes into aqueous solution, and the nitrogen in the insoluble residue after precipitation with lead indicates the total albumen, both soluble and insoluble.

In addition to the foregoing precipitants, some of the group reagents for alkaloids-phosphomolybdic, phosphotungstic acid, potassiomercuric iodide, etc.-also throw down albuminous substances (§63). Phosphotungstic acid precipitates peptones, and might therefore be used for their estimation in vegetable infusions previously freed from albuminous substances by coagulation or precipitation with lead. ${ }^{1}$

${ }^{1}$ See Schulze and Barbieri, Landwirthsch. Versuchsst. xxvi. 213, 230, 234, 1881 (Journ. Chem. Soc. xl. 312) ; Chem. Centralblatt, 714, 731, 747, 761, 1881; Defresne, Repert. de Pharm. viii. 453, 1881; Hofmeister, Zeitschr. f. phys. Chem. iv. 253, 1880.

From the results recently obtained by Schulze and Barbieri, it appears probable that peptones are of far more frequent occurrence than could have been anticipated. As plants contain peptonizing ferments, the possibility must not be ignored of peptones being produced during the preparation of aqueous infusions ; they are also occasionally found ready formed in plants. The following are the more important properties of peptones : They yield with water solutions from which they are precipitated by alcohol, and redissolved by the addition of water. Estimation, however, by precipitation with alcohol is said to yield unsatisfactory results. In aqueous solution they are not coagulated by warming, nor are they thrown down by nitric acid, alum, ferrocyanide of potassium, or acetate of lead, but they are precipitated by tannin ; and in the presence of neutral salts (sulphate of magnesia, etc.) the separation is often very complete. Peptones are precipitated, as above stated, by phosphotungstic acid, and this takes place in an acetic acid solution; a property that enables us to separate them from other nitrogenous substances thrown down by the same reagent from solutions containing a mineral acid. The most important reaction of peptones is the so-called biuret reaction. An aqueous solution of a peptone assumes a pure red colour on the addition of caustic soda and very dilute solution of sulphate of copper (avoiding excess); Fehling's solution produces the same effect. The following might temporarily be recommended as a suitable method for the detection of peptones: The (fresh) material to be examined is triturated with sand and water, strained, washed with water and pressed. The liquors are united, acidified with acetic acid, warmed and filtered from the coagulum. From the filtrate any albuminoids remaining in solution are precipitated by the addition of acetate of lead, or, better, by warming with basic acetate and hydrate of lead, and filtered off. The clear liquid is then rendered strongly acid with sulphuric acid, and the peptone precipitated 
Precipitation by phenol and calculation from the nitrogen contained in the precipitate has been recommended by Church ${ }^{1}$ for the estimation of the albuminoids in vegetable infusions, in the presence of amides, etc.

My experience in precipitating albumen, etc., with phenol compels me to doubt the possibility of always obtaining complete separation by this means. Sestini has also expressed himself to the same effect.

\$233. Extraction with Dilute Acid.-It has already been observed that the residue of a vegetable substance, after exhaustion with water, yields albuminous substances to dilute alkali. The same is the case with dilute acid $(2 \cdot 12$ per cent. $\mathrm{HCl})$, the substances extracted being gluten, fibrin ( $\$ 235)$, gliadin, mucedin, etc. But the albuminoids brought into solution by these two solvents do not appear to be always identical; at least Wagner found that the amount removed by dilute alkali (after exhaustion of the material with water) did not coincide with that extracted by acid. (Compare also $\$ \S 111,106)$. It might nevertheless in many cases be desirable to ascertain to what extent the substances allied to albumen resist the action of water, dilute alkali (cf. §226) and dilute acid respectively.

In estimating the value of certain vegetable substances as foods, it will often be found desirable to determine what proportion of proteids are dissolved by the combined action of pepsin and hydrochloric acid after the material has been exhausted with water.

From experiments that have been made in this direction it would appear that hydrochloric acid and pepsin dissolve more than the former alone. ${ }^{2}$ In making such estimations I should recommend 100 cc. of water, 1 gram of 33 per cent. hydrochloric acid, and $0 \cdot 1$

by phosphotungstic acid. The precipitate is filtered off as rapidly as possible, washed with 5 per cent. sulphuric acid and transferred whilst still moist to a mortar. It is then triturated with excess of hydrate of baryta, warmed for a short time and filtered. If the filtrate is colourless, the biuret-test can be applied; if yellow, it can frequently be decolourized by adding a little acetate of lead, and filtering off from the precipitate thus produced. Animal charcoal should not be used, as it absorbs peptone. Schulze and Barbieri, who proposed the foregoing method, have obtained approximate quantitative results colorimetrically.

1 Landwirthsch. Versuchsst. xxvi. 193 (Journ. Chem. Soc. xxxviii. 588). See also Sestini, loc. cit.

${ }^{2}$ See Kessler, Versuche über die Wirkung des Pepsins auf einige animal. u. vegetab. Nahrungsmittel. Diss. Dorpat, 1880. 
of good pepsin to be taken for every 2 grams of finely powdered substance. Starch, if present in large quantity, might with advantage be previously converted into maltose and dextrin by boiling, cooling to $40^{\circ}$, and digesting for four hours at that temperature, after adding 0.005 gram of active diastase.

\$234. Extraction with Spirit.-Some of the albuminoids insoluble in water attract our attention by their solubility in spirit, as, for instance, those known as glutenfibrin, gliadin (or vegetable gelatine), and mucedin. In seeds only have these three substances been detected with certainty; they remain undissolved when the material containing them is treated with water, or, at most, the mucedin alone is partially taken into solution. They would be removed, however, by the dilute alkali used for the extraction of the glutencaseïn ( $\$ 226$ ), and it is advisable therefore, in looking for these substances, to treat the material with spirit previously to extracting the glutencasein with alkali. Part, however, of the glutenfibrin and a little gliadin would be left undissolved, and would be subsequently found with the casein $(\$ 226)$. The spirit should be used cold, and should be of a strength of about 60 to 80 per cent. The maceration must extend over a considerable period, and the spirit be renewed several times. The united extracts are distilled until the strength of the spirit is reduced to 40 to 50 per cent. (not less). On cooling, a clear slimy mass separates, consisting principally of glutenfibrin mixed with a few flocks of glutencasein and possibly fat (which is, however, better removed by petroleum spirit before treating with alcohol). If the majority of the spirit is distilled off from the clear liquor a second precipitate will form, consisting principally of gliadin and mucedin, and a further quantity of the same two substances (impure) can be obtained by neutralizing the filtrate with a little potash and concentrating.

All these precipitates are triturated with absolute alcohol until they become hard and solid.1 Fat, if present, is removed by treatment with ether.

We are as yet unacquainted with any method of separating the glutenfibrin, gliadin, or mucedin for quantitative determination. We must therefore content ourselves with making a total estima-

1 The spirit dissolves a little glutenfibrin, which can subsequently be precipitated by ether. 
tion, and applying a few qualitative tests to show the presence of one or more of the substances referred to.

$\S 235$. Properties: Glutenfibrin - Glutenfibrin is insoluble in water and in absolute alcohol, but dissolves easily in warm 30 to 70 per cent. spirit, separating again on cooling. ${ }^{1}$ It is also taken up by cold 80 to 90 per cent. spirit. Prolonged boiling with water converts it into a gelatinous substance insoluble in spirit, acids, or alkalies. Glutenfibrin dissolves with facility in cold dilute acids (acetic, citric, tartaric, hydrochloric), and in alkalies; with ammonia, lime- and baryta- water it gelatinizes. It is precipitated from both acid and alkaline solutions on neutralizing, and is also thrown down by acetate of copper. ${ }^{2}$

Gliadin is characterized by its tough, slimy consistency. It is sparingly soluble in cold water; a considerable quantity dissolves on boiling, but, like glutenfibrin, it undergoes simultaneously a partial decomposition. Gliadin is insoluble in absolute alcohol, but dissolves in 60 to 70 per cent. spirit, both cold and warm (especially freely in the .latter). In general it resembles glutencasein in its behaviour to dilute alkalies and acids, but ammonia, lime- and baryta- water dissolve it. Boiled with concentrated hydrochloric acid it yields a bluish-brown solution. It is precipitated by acetate of copper, but not by mercuric chloride. Attention has already been directed (\$224) to the high percentage of nitrogen in gliadin.

Mucedin is far less tough and elastic than gliadin, and is more easily soluble in 60 to 70 per cent. spirit. It is precipitated from a cold solution by 90 to 95 per cent. spirit in flocks or friable masses (solutions of gliadin become milky); stirred up with water it yields a cloudy mucilaginous liquid, which clears again on standing; but, if warmed, the aqueous solution becomes cloudy and remains so for a considerable period, till finally a flocky mass separates which is only partially soluble in acetic acid and spirit.

${ }^{1}$ On concentrating such solutions the glutenfibrin forms a skin on the surface of the liquids, which dissolves again on stirring. Gliadin and mucedin do not exhibit this peculiarity.

${ }^{2}$ Glutenfibrin agrees with maize-fibrin in most of its properties; the latter contains only 15.5 (instead of 16.9 ) per cent. of nitrogen, and is insoluble, or only partially dissolved, by dilute acetic, citric, tartaric and oxalic acids. Zander has recently reported on another albuminous substance soluble in spirit ('Chemisches über die Samen des Xanthium Strumarium.' Diss. Dorpat, 1881). 
In its other properties it agrees fairly well with gliadin. (Compare also $§ 237$.)

$\S 236$. Gluten.-Glutencaseï, glutenfibrin, gliadin, and mucedin are the principal constituents of the so-called gluten which possesses such importance as a food. An estimation of total gluten is generally made by rubbing down 10 to 20 grams of the meal to a paste with water, transferring to a fine linen cloth, and washing with distilled or rain-water until the washings, on standing, deposit only traces of starch. The mass is then pressed, scraped from the cloth, and dried on watch-glasses, finally at a temperature of $115^{\circ}$ to $120^{\circ}$; it should then be powdered and dried again until the weight is constant. In this method of estimating gluten it will be found advantageous to add a weighed quantity (1 to 2 grams) of purified bran, the weight of which is afterwards, of course, to be deducted from that of the total gluten. ${ }^{1}$

According to Benard and Girardin, ${ }^{2}$ the amount of gluten found varies if the mixture is allowed to stand before washing with water. It would be advisable to begin washing about three hours after mixing the meal with water.

§237. Substances dissolved by Dilute Alkali, not precipitated by Acid and Spirit.-In estimating metarabic acid and albuminous substances sparingly soluble in water, as directed in $\$ \$ 103$ and 206, it will not unfrequently be observed that the total substances extracted by dilute alkali are considerably in excess of those precipitated by acid and alcohol. A part of the former, therefore, must still remain in solution, and will be recovered, together with acetate of sodium, by evaporating the filtrate (\$ 107). We may expect to find here the constituents of gluten (including gliadin) and products of their decomposition. After distilling off the majority of the spirit, they might be precipitated with acetate of copper, and estimated as directed in $\S 231$.

The substances not precipitated by this reagent are probably allied to, or derived from, vegetable mucilage; they may be estimated by removing the excess of copper with sulphuretted hydrogen, evaporating to dryness and weighing, deducting the acetate of soda present.

With regard to the latter, I may observe that it cannot be calculated from the amount of soda used, but must be estimated by

1 Compare Archiv d. Pharm. cxcv. 47, 1871.

2 Journ. de Pharm. et de Chim. [5], iv. 127, 1881. 
incinerating a portion of the dried residue, and calculating from the carbonate of soda in the ash. In many analyses made in my laboratory, the amount of soda in solution has been found to be much smaller than was expected from calculation; part of it was evidently retained in the insoluble residue.

$\S 238$. Other Nitrogenous Substances.-We possess hardly any knowledge at all of the nitrogenous substances that are not dissolved by water, alcohol, or alkali. I have already stated (\$234) that they may sometimes be extracted by hydrochloric acid and pepsin, but Treffner's researches on the chemical composition of the mosses, alluded to in $\S 106$, prove that this is not always the case. I will here only remark that, in estimating the nutritive value of a plant, such substances cannot, without further consideration, be considered as albuminoids.

\section{AMINES AND THEIR COMPOUNDS.}

\$239. Monamines.-According to A. W. Hofmann, monamines may be distinguished from other amines by means of the isonitrilereaction, as the latter do not evolve the characteristic odour of that compound when warmed with alcoholic potash and chloroform.

Another reaction for monamines consists in warming an alcoholic solution with bisulphide of carbon, by which a sulphocarbamide of the base is produced. This compound, when heated with an aqueous solution of mercuric chloride (not in excess) develops an odour of oil of mustard. ${ }^{1}$

$\S 240$. For the separation of ethylamine from diethyl- and triethylamine by means of anhydrous ethyloxalate, see A. W. Hofmann ;2 the author subsequently availed himself of the method in separating the methyl bases. Carey $\mathrm{Lea}^{3}$ recommends picric acid for the ethyl bases.

In Hofmann's method the ethylamine is converted into diethyloxamide, which can be recrystallized from water, and yields ethylamine by distillation with potash.

Diethylamine yields under the same conditions oily ethylic diethyloxamate, which can be purified by distillation (boils at $260^{\circ}$ ), and converted by potash into diethylamine.

1 Ber. d. d. chem. Ges. iii. 767, 1870.

2 Journ. f. pract. Chem. Ixxxiii. 191, 1861 ; Comptes rendus, Iv. 749, 1862.

${ }^{3}$ Chem. Centralblatt, 76, 1863. 
Triethylamine is not attacked by ethylic oxalate, and can be separated from diethyloxamide and ethylic diethyloxamate by distillation (B.P. $91^{\circ}$ ).

The three corresponding methyl bases behave in an exactly similar manner. Trimethylamine boils at $4^{\circ}$ to $5^{\circ}$ and can easily be separated from the crystalline methylethyloxamide and the liquid ethylic dimethyloxamate (B.P. $240^{\circ}$ to $250^{\circ}$ ) by distillation.

$\S 241$. Estimation:-Sachsse and Kormann ${ }^{1}$ have published a method for the approximate estimation of amides, based upon their decomposition by nitrous acid with liberation of nitrogen; the

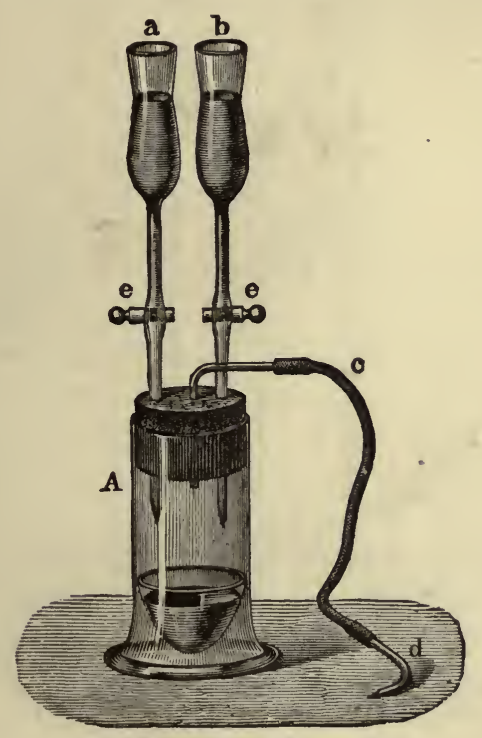

Fig. 10.

latter gas is collected and measured, and from it the amount of amide originally present is calculated.

The apparatus used for the estimation is shown in Figs. 10 and 11. The generating vessel $\mathrm{A}$ is of about 50 to $60 \mathrm{cc}$. capacity, and closed with an indiarubber cork bored with three holes; through these there pass two funnel-tubes, $a$ and $b$, and a bent delivery tube $c$, to which is attached, by means of a long indiarubber tube,

${ }^{1}$ Landwirthsch. Versuchsst. xvii. 321 (Journ. Chem. Soc. xxvii. 784); Zeitschr. f. anal. Chem. xiv. 380, 1875. 
a curved glass point $d$. About 6 cc. of a concentrated aqueous solution of nitrite of potassium (free from carbonate), together with nearly an equal quantity of water, is introduced into the generating vessel. The lower parts of the funnel-tubes, that is up to a little above the tap, say about $e$, are also filled with water, so as to displace the atmospheric air. Dilute sulphuric acid is now poured into one funnel, and a weighed quantity of the amide dissolved in water into the other, taking care not to allow any bubbles of air to adhere to the sides.

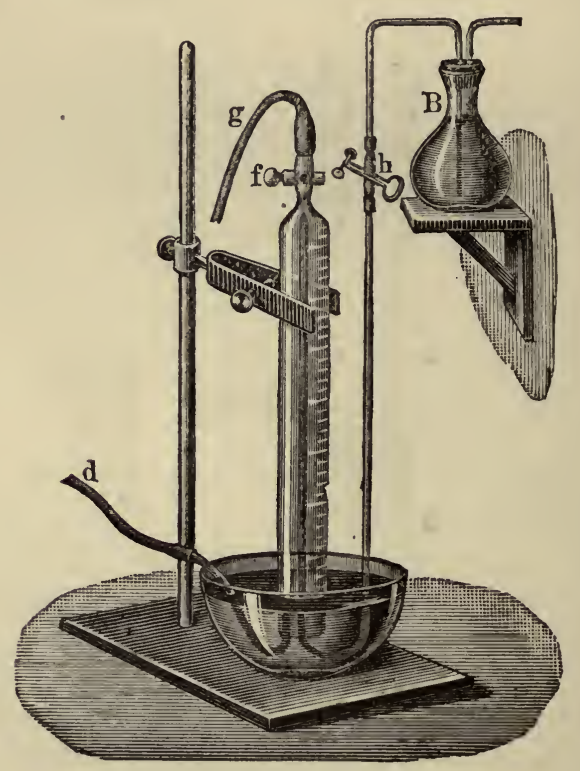

Fig. 11.

The atmospheric air in the apparatus has now to be displaced, and this is effected by running sulphuric acid, little by little, into the nitrite solution, by which nitrous acid and nitric oxide are evolved. To ascertain if the displacement is complete, 5 to 10 cc. of the gas from the generating vessel are allowed to pass into the measuring tube (Fig. 11) previously filled with solution of ferrous sulphate. Not more than $0 \cdot 1 \mathrm{cc}$. should remain unabsorbed. Fresh ironsolution may be introduced, if necessary, from the flask $B$, as subsequently described. The apparatus is now ready for the commencement of the actual experiment. The measuring-tube, stand- 
ing in a pneumatic trough, should be capable of holding 50 to 60 cc., and be graduated to $0.2 \mathrm{cc}$. It is filled with the iron-solution contained in B by opening the clip $h$ and blowing through the shorter bent tube in B; by this means the solution can be run into the pneumatic trough; on opening $f$ and sucking at $g$ the solution rises in the tube until it reaches and passes $f$, which should then be closed.

After replacing the clip $h$, the bent point $d$ is introduced under the measuring tube and the solution of the amide allowed to run from the second funnel-tube into the generating vessel, rinsing with a little water, but keeping the tube from $e$ downwards full of liquid. Small quantities of sulphuric acid are allowed to run into tie generating vessel from time to time, when the evolution of gas becomes sluggish, taking care that the measuring-tube always contains sufficient strong solution of ferrous sulphate; this can be ensured by frequently opening the clip $h$ and allowing the solution from $B$ to run into the measuring-tube. The end of the decomposition is recognised by the liquid in A assuming a permanent blue colour from excess of nitrous acid. The remainder of the gas is then driven out by filling the entire apparatus with water through the second funnel-tube until it flows into the measuringtibe through $d$. The delivery-tube is now removed, and the vhole of the nitric oxide absorbed by the introduction of fresh iron-solution. After closing the clip $h$, the delivery-tube from B is drawn out of the measuring-tube, and the latter transferred to a deep cylinder, where the iron-solution is removed as far as possible and replaced by caustic soda to absorb carbonic acid. When this has been effected, the measuring-tube is lowered in the cylinder until both liquids have the same level. The volume of gas is now read off, reduced to $0^{\circ}$ and from it the amount of amide originally present calculated, deducting $1 \mathrm{cc}$. as unavoidable error caused by the atmospheric air mixed with the nitrogen ; 28 parts by weight of nitrogen indicate 150 of crystallized asparagine, 131 of leucine, and 181 of tyrosine. (\$§ 191, 192).

$\S 242$. Amidic Acids. - The amidic acids referred to in $\S 101$ are freely soluble in water and 50 per cent. spirit, requiring considerable quantities of strong alcohol for precipitation, so that in this respect they resemble such substances as dextrin, levulin, etc. They are precipitated therefore with, or in the place of, dextrin and the like, but differ from these bodies in containing nitrogen. 
The precipitate obtained as dextrin (cf. $\S \S 76,198,199$ ) must be tested for nitrogen, and if much is found, experiments must be made to ascertain whether any one of the following substances is present. It may sometimes be approximately estimated, if found, by mixing the aqueous solution with alcohol till it contains about 50 to 60 per cent., filtering, evaporating the filtrate to a syrupy consistence, and now precipitating with 5 to 6 volumes of absolute alcohol. From the amount of nitrogen in the precipitate the quantity of amidic acid present may be calculated.

Cathartic Acid occurs in senna, in the bark of Rhamnus frangula, and probably also in rhubarb. ${ }^{1}$ It is a glucoside, yielding by its decomposition sparingly soluble cathartogenic acid and $34 \cdot 1$ per cent. of glucose. According to Kubly, cathartic acid ${ }^{2}$ contains 1.48 to 1.51 per cent. of nitrogen, cathartogenic acid 2.46 per cent. The latter is easily produced by heating an aqueous solution of cathartic acid with access of air ; in fact, that substance decomposes with great facility in the presence of bases and air. In senna and rhubarb it is contained chiefly in combination witi bases (the alcohol precipitate containing 4 to 5 per cent. of ash); but in Rhamnus frangula it appears to occur, partly at least, in the free state. It is a strong purgative.

Husson $^{3}$ estimates the quality of a rhubarb by ascertaining the amount of iodine an infusion is capable of absorbing; but Greenish $^{4}$ has shown that this method does not yield reliable results.

Sclerotic Acid 5 is a constituent of ergot, and contains about $4 \cdot 2$ per cent. of nitrogen, but no sulphur; its activity is not destroyed by acids, etc., if in contact with them for a short period only. In solubility it resembles cathartic acid. Its action, when injected subcutaneously into frogs and other animals, is that of a powerful

1 Compare Kubly, 'Ueber das wirksame Princip und einige andere Best. d. Sennesblätter,' Diss. Dorpat, 1865, and Pharm. Zeitschr. f. Russland, iv. 429, 465. On Rhamnus frangula, see also Pharm. Zeitschr. f. Russland, v. 160, 1866. On rhubarb, ibid. vi. 603, 1867 ; xvii. 65, 97, 1878 (Pharm. Journ. and Trans. [3], ix. 813, 933, 1879).

2 Probably also sulphur; cathartic acid from Rhamnus frangula bark contains less nitrogen.

3 Union Pharm. 99, 1875 (Year-book Pharm. 344, 1875).

4 Pharm. Journ. and Trans. [3], ix. 813.

${ }^{5}$ Compare Dragendorff and Podwissotzki, Archiv f. exper. Patholog. und Pharm. 153, 1876 ; Sitz-Ber. d. Dorpater Naturf. Ges. 109, 392, 1877 (Pharm. Journ. and Trans. [3], vi. 1001). 
poison. ${ }^{1}$ It is precipitated by tannin and basic acetate of lead, and from concentrated solutions also by chlorine-water and phenol. It does not share with albuminoids the reactions mentioned in $\S 92$.

On keeping ergot for any length of time, part of the sclerotic acid appears to be converted into an allied substance containing 6.6 per cent. of nitrogen, which has been named scleromucin. It can be extracted with warm water, but requires less alcohol for precipitation than sclerotic acid. Diffused in water whilst still moist, it forms a mucilaginous liquid; but once dried, it is not dissolved by cold water, and not with facility by warm. It resembles sclerotic acid in its action and other properties.

\section{STARCH, LICHENIN, WOOD-GUM, ETC.}

§ 243. Starch.-Starch is not, as is well known, a homogeneous substance, but it is nevertheless usual, and very properly so, to estimate the whole of the carbohydrates of which it is composed as directed in $\$ 8113$ to 115 . Formerly three principal constituents of starch were generally distinguished : first, one striking a blue colour with iodine, and passing into solution when starch is triturated with powdered glass and water-soluble starch, amidulin, \& amylon (Béchamp); secondly, a substance characterized by its insolubility in cold water, solubility in saliva, etc., and by the blue colouration it yields with iodine, granulose, the principal constituent of all starch ; and thirdly, cellulose, which, in the form of a membrane, gives to the starch grain its particular shape, is coloured yellow by iodine (after boiling with water, violet), and is converted by chloride of zinc into a substance that is tinged blue by the same reagent.

Some years ago Nägeli ${ }^{2}$ stated that in his opinion there existed two different modifications of amylon, which he called blue

1 From 0.03 to 0.04 gram produces in frogs a swelling of the skin and almost complete paralysis, commencing at the hinder extremities. Irritants produce no effect, and indeed the animal gives no other sign of life than an occasional feeble contraction of the heart. Although its condition may appear to improve in the course of five to seven days, it sometimes succumbs to a relapse.

${ }^{2}$ Annal. d. Chem. und Pharm. clxxiii. 218, 1874 (Journ. Chem. Soc. xxviii. 55. See also Musculus, Annal. de chim. et de Phys. ii. 385, 1874 (Pharm. Journ. and Trans. [3], v. 3) ; Musculus and Gruber, Journ. de Pharm. et de Chim. xxviii. 308, 1878 (Journ. Chem. Soc. xxxiv. 778); Bondonneau, Repert. de Pharm. iii. 231, 1875 (Journ. Chem. Soc. xxix. 365) ; Journ. de Pharm. et de Chim. xxiii. 34, 1874 ; Béchamp, ibid. 141. 
and yellow, according to the colour they yielded with iodine. These two were connected by intermediate modifications striking violet, reddish and reddish yellow colours with iodine, differences which are probably referable to variations in the density. In accordance with this theory the several modifications vary in the resistance they offer to solvents and chemical agents. As the blue modification is the most easily attacked, it might be considered to be that of lowest density. It is followed by the violet, red, etc., in succession up to the yellow, the densest form of which shows a great resemblance to cellulose. When starch is boiled the blue modification passes into solution, carrying with it a little of the yellow. If the former is removed by allowing it to decompose, the yellow modification separates out. From a solution of the latter, prepared by prolonged boiling with water and concentrating, crystals of amylo-dextrin can be obtained, which are coloured yellow by iodine.

The bodies above referred to occur in different proportions in the different varieties of starch, and the amount of either present might possibly be found to be characteristic of the starch under examination. It might, for instance, be ascertained by comparative experiments how long the action of an acid of certain strength must be continued before the blue or red colouration with iodine ceases to be produced. For the isolation of the yellow modification, formerly called cellulose, $\gamma$ amylon (Béchamp), I have recommended digestion at a temperature not exceeding $60^{\circ}$, with 40 parts of a saturated solution of chloride of sodium containing 1 per cent. of hydrochloric acid, and washing with water and dilute spirit. I have thus obtained 3.4 per cent. from arrowroot, $2 \cdot 3$ per cent. from wheat-starch, and $5 \cdot 7$ per cent. from potato-starch.

$\S 244$. Hydrocellulose.-A blue colouration of the cell-uall is frequently noticed when sections of vegetable substances are moistened with iodine water. It was probably this reaction that gave rise to the theory that a modification of cellulose could occur striking a blue colour with iodine.

I do not concur in this view; in fact, I am convinced that in such cases the cell-wall in question contains besides cellulose, which is characterized by its power of resisting the action of chlorate of potash and nitric acid, other carbohydrates (amyloid), probably, at least in part, of the composition $\mathrm{C}_{12} \mathrm{H}_{22} \mathrm{O}_{11}$, agreeing therefore in this respect with arabic acid, pararabin, etc. Whether these 
carbohydrates are hydrocelluloses, such as are formed from cellulose by the action of concentrated sulphuric acid or chloride of zinc, is a matter for further inquiry. If the treatment with the above oxidizing mixture of chlorate of potash and nitric acid ( $(119)$ is continued long enough, such substances are always destroyed. Some of them are soluble in boiling water. This is the case with one contained in the asci of certain lichens (Cetraria), etc., from which it is extracted, together with lichenin, by boiling with water; hence the erroneous idea that the lichenin itself was coloured blue by iodine. ${ }^{1}$

Berg's researches have shown that if a decoction of the lichenin be allowed to gelatinize by cooling, cut into pieces and macerated in distilled water, the whole of the substance that strikes a blue colour with iodine passes into solution, from which it can be isolated by precipitation with alcohol, although impure and not free from ash. After drying it is to a great extent insoluble in water, and is converted into sugar by boiling with dilute hydrochloric acid (4 per cent. of acid of sp. gr. 1-12) for a period of two hours, a change which is not effected by pure water. The glucose produced is dextro-rotatory, and as the decomposition takes place tolerably smoothly, the amount of the substance, which we may temporarily call lichen-starch, can be determined by estimating the sugar thus formed. Lichen-starch dissolves tolerably easily in ammonia of sp. gr. 0.96 , and is precipitated from this solution by spirit. It appears to be more difficultly soluble in dilute alkalies, and is not converted into sugar by diastase or saliva.

$\S 245$. Lichenin.-Lichenin is characterized by its property of gelatinizing, which is exhibited by a solution containing 1 in 60 . It is insoluble in cold water, alcohol, and ether; boiling water dissolves it, as do also ammonio-sulphate of copper and concentrated (20 to 30 per cent.) potash. From its solution in strong potash it can be precipitated by alcohol in the form of a potassium-compound containing up to 10 per cent. of alkali. Concentrated hydrochloric acid also dissolves it, but with simultaneous (partial) decomposition. When boiled with dilute acid it is converted with even more facility than lichen-starch into a dextro-

1 Compare Berg, 'Zur Kenntniss des in Cetraria islandica vork. Lichenins und iodbläuenden Stoffes,' Diss. Dorpat, 1872. From Berg's experiments it would appear that the formula $\mathrm{C}_{6} \mathrm{H}_{10} \mathrm{O}_{5}$ would indicate the composition of lichen-starch better than $\mathrm{C}_{12} \mathrm{H}_{22} \mathrm{O}_{11}$; the same is true of lichenin. 
rotatory fermentable sugar, so that this method may be adopted for its estimation. Ammonia dissolves it with difficulty, and it undergoes but little change when heated with potash in sealed tubes ( $(115)$.

Gelose, ${ }^{1}$ the gelatinizing constituent of many algæ, agrees with lichenin in most of its properties, but is insoluble in ammoniosulphate of copper, and is less easily converted into sugar. By decomposition with dilute acids, arabinose (lactose) is produced in place of the glucose yielded by lichenin. The gelose appears to be accompanied, at least in Sphærococcus lichenoides, by a carbohydrate $^{2}$ soluble in dilute hydrochloric acid, but differing from pararabin $(\$ 112)$ in yielding glucose when boiled with an acid.

$\S 246$. Wood-gum.-Thomsen ${ }^{3}$ found that when ligneous tissue, previously exhausted with water, spirit, and very dilute alkali, was macerated with caustic soda of sp. gr. 1·1, a substance was extracted, the composition of which he ascertained to be $\mathrm{C}_{6} \mathrm{H}_{10} \mathrm{O}_{5}$, and which he named wood-gum. It can be isolated from its solution in soda by acidifying and adding alcohol. When once dried, cold water will not redissolve it; this is, however, effected by boiling. It is precipitated by basic acetate of lead, is converted into glucose by boiling with a dilute acid, and is not coloured blue by iodine. An alkaline solution is lævo-rotatory. It differs from lichenin in not possessing the power of gelatinizing, from metarabin in not being dissolved (when dry) by 0.1 per cent solution of soda.

A similar substance was obtained by $\mathrm{Pfeil}^{4}$ from parenchymatous tissue (agreeing, however, in composition better with the formula $\mathrm{C}_{12} \mathrm{H}_{22} \mathrm{O}_{11}$, a hydrocellulose), by Treffner from mosses, and by Greenish from algæ.

\section{CELLULOSES, LIGNIN, AND ALLIED SUBSTANCES.}

$\S 247$. Celluloses, etc.-Frémy and Terreil ${ }^{5}$ assume that woody tissue is chiefly composed of three different substances, which they distinguish as cellulose, incrusting substance, and cuticular sub-

1 Compare Morin and Porumbaru, Comptes rendus, xc. 924, 1081, 1880 (Year-book Pharm. 120, 121, 1881).

${ }^{2}$ Greenish, Archiv d. Pharm. [3], xx. 241.

3 Journ. f. pract. Chem. [2], xix. 146, 1879 (Year-book Pharm. 99, 1880).

${ }^{4}$ Loc. cit.

5 Journ. de Pharm. et de Chim. vii. 241, 1868. 
stance. The first is said to be the only one capable of resisting the action of chlorine-water; it can be isolated by the method detailed in $\S 116$. The authors overlook the fact that several units per cent. of a substance probably isomeric with cellulose (? intercellular substance), removable by chlorate of potash and nitric acid, are left associated with the cellulose.

The cuticular substance alone is said to be insoluble in a mixture of 1 eq. of sulphuric acid with 4 eq. of water ; it can be isolated by treatment with acid of that strength, followed by washing with pure water and dilute alkali.

The incrusting substances are estimated by difference.

In a more recent publication, the authors observe that the following are the principal substances they would expect to find in tissue previously exhausted with indifferent solvents :

Cellulose, soluble in ammonio-sulphate of copper.

Paracellulose, insoluble in the same until after it has been acted upon by acids.

Metacellulose (fungin) insoluble in ammonio-sulphate of copper.

All three modifications of cellulose are soluble in $\mathrm{H}_{2} \mathrm{SO}_{4}, 2 \mathrm{H}_{2} \mathrm{O}$. (Compare also $\S 248$ ).

Vasculose, insoluble in $\mathrm{H}_{2} \mathrm{SO}_{4}, 2 \mathrm{H}_{2} \mathrm{O}$, and in ammonio-sulphate of copper; soluble in alkalies only under increased pressure, and decomposed by treatment with chlorine-water, followed by washing with dilute alkalies.

Cutose, insoluble in $\mathrm{H}_{2} \mathrm{SO}_{4}, 2 \mathrm{H}_{2} \mathrm{O}$, and in ammonio-sulphate of copper, but soluble in alkalies under the ordinary pressure.

Pectose, convertible by acids into soluble pectin. ${ }^{1}$

I would observe that the substance designated as vasculose (formerly called incrusting substance), agrees in the main with my lignin (§ 116). Lignin cannot, unfortunately, be separated from cellulose without decomposition, and it is therefore impossible to adduce direct proof that it does not consist of a mixture of several chemical individuals. Nevertheless, I think it probable that in some instances the cellulose is accompanied by a single definite substance, 'lignin.' Stackmann ${ }^{2}$ exhausted vegetable substances rich in lignin with the indifferent solvents already alluded to, as well as with dilute soda and dilute acid, and then determined the approximate composition of the lignin by making

${ }_{1}$ Comptes rendus, Ixxxiii. 1136. (Journ. Chem. Soc. xxxi. 229).

2 'Studien über die Zusammensetzung d. Holzes.' Diss. Dorpat, 1878. 
an ultimate analysis of the material that had been thus treated, both before and after the action of chlorine-water. Several varieties of wood yielded tolerably concordant results. The lignin of dicotyledons appeared to contain between $53 \cdot 1$ and $59 \cdot 6$ per cent. of carbon, 4.4 and 6.3 per cent. of hydrogen, 34.1 and 38.9 per cent. of oxygen; the majority of his results agree very well with Fr. Schulze's $1(\mathrm{C}=55 \cdot 5, \mathrm{H}=5 \cdot 8, \mathrm{O}=38 \cdot 6)$; but German walnut and mahogany show a little variation, probably due to the larger amount of foreign substances they contain. All the dicotyledonous woods examined by Schulze and Stackmann must have contained at least one substance in notable quantity, viz. wood-gum, which was not discovered until after the publication of Stackmann's work. Experiments made by Schuppe, ${ }^{2}$ at my suggestion, showed that poplar wood contained $3 \cdot 25$ per cent. of wood-gum, mahogany $3 \cdot 37$, American walnut 4.56 , German walnut, $6 \cdot 32$, oak $6 \cdot 03$, and alder $7 \cdot 09$. Deducting the wood-gum present, the average amount of lignin in the majority of woods is about 17 per cent. (mahogany 20.4), and its mean composition, 60.56 per cent. C, 4.66 per cent. $H$, and 34.80 per cent. $O$. In this respect it approaches catechin, many tannins and phlobaphenes, and agrees fairly well with the lignin of coniferous woods which contain no wood-gum. Stackmann found about the same quantity of lignin in the wood of gymnosperms as Schuppe did in that of angiosperms, viz. 16 to 17 per cent.

Koroll ${ }^{3}$ found the lignin of sclerenchymatous tissue (hazel-nut, walnut) to contain from 51.5 to 54.2 per cent. of carbon, 4.8 to $5 \cdot 5$ per cent. of hydrogen, and $40 \cdot 1$ to $44 \cdot 7$ of oxygen, and estimated its quantity at $14 \cdot 3$ to $15 \cdot 7$ per cent. A substance resembling wood-gum also occurs in the sclerenchymatous tissue of nut-shells. Bast-fibres (lime and elm) yielded him 14.5 to 15.8 per cent. of lignin, containing 53.6 to 54.9 per cent. of carbon, 4.9 to 6.0 per cent. of hydrogen, and 40.1 to 40.4 per cent. of oxygen.

On the other hand, from the outer birch-bark (rich in cuticular substance,) chlorine-water extracted 11 per cent. of a substance of an entirely different composition; viz. $\mathrm{C}=72 \cdot 7, \mathrm{H}=7 \cdot 8$, $\mathrm{O}=19 \cdot 4$. (Cf. $\S 250$.)

1 'Beitr. z. Kenntniss d. Lignins.' Rostock, 1856.

2 Beiträge z. Chemie d. Holzgewebes. Diss. Dorpat, 1882.

3 'Quant. chem. Unters. über d. Zusammensetz. d, Kork-, Bast-, Sclerenchym, und Markgewebes.' Diss. Dorpat, 1880. 
The tissue of turnip, chicory-root, and elder-pith, which is principally parenchymatous, yielded hardly anything to chlorinewater. Pfeil also came to a similar conclusion with regard to the tissue of apples. ${ }^{1}$

The substance formerly known as suberin is in part the cuticular substance just alluded to ; it should, however, be observed that under this name less recent authors understood a mixture of fat, wax, tannin, etc. ${ }^{2}$ Siewert has published a minute investigation of the substances that accompany suberin, but not of the suberin itself ; our knowledge of that substance is but very insufficient, and I can only state that it is not dissolved by the usual solvents, that it is more easily attacked by certain oxidizing agents than lignin, but is more difficult to remove completely by digestion with chlorine-water. Nitric acid of sp. gr. 1.3 attacks it very energetically; and with an acid of sp. gr. $1 \cdot 4$ the action may be so violent as to cause ignition. It resists chromic acid more powerfully than lignin. Whether suberin really yields the ceric and. suberic acids that have been obtained by the decomposition of cork is still a matter of uncertainty.

Siewert estimates the amount of suberin in cork at 90 per cent.; but I think this is too high. I feel convinced that the residue he speaks of as suberin must have contained a considerable quantity of true cellulose. (Koroll found 50 per cent. in the outermost parts of birch-bark.)

In my opinion, the hardening substance of many woody fungi is possibly identical with suberin. ${ }^{3}$

For the microchemical characters of cutin, lignin, etc., see also Vogl ${ }^{4}$ and Poulsen. ${ }^{5}$ (See also $§ 249$.)

The remarkably constant proportion existing between the amount of cellulose and lignin, etc., present in varieties of wood, raises the question whether these two substances do not occur in combination with one another. The attempt has frequently been made to regard the substance of the cell-walls of lignified tissue

${ }^{1}$ Loc. cit.

2 Compare Siewert, Zeitschr. f. d. ges. Naturw., xxx. 129 ; Journ. f. pract. Chem. civ. 118, 1868. See also Höhnel, Sitz.-ber der phys. math. K. d. Akad. d. W. in Wien, 1877 ; Bot. Ztg. 783.

3 Compare my 'Chem. Unters. eines an Betula alba vork. Pilzes.' Diss. Petersburg, 1864.

4 Zeitsch. d. österr. Apotheker-Ver. 1867, 16, 34, 60.

5 ' Botanisk Mikrokemi.' Kjöbenhavn, 1880. 
as a special chemical compound (gluco-lignose, gluco-drupose of Erdmann). Erdmann assumes that it is decomposed by hydrochloric acid with production of glucose, together with lignose or drupose, and that with nitric acid it yields cellulose, whilst the lignose or drupose undergoes further decomposition. Bente, ${ }^{1}$ who doubts the existence of gluco-drupose, shows that wood-cells (? lignin) yield pyrocatechin when fused with potash.

$\S 248$. Cellulose.-The cellulose obtained from various plants in the manner indicated does not appear to be invariably of the composition $\mathrm{C}_{6} \mathrm{H}_{10} \mathrm{O}_{5}$. That isolated by Stackmann from coniferous wood was represented by the formula $5\left(\mathrm{C}_{6} \mathrm{H}_{10} \mathrm{O}_{5}\right)+\mathrm{H}_{2} \mathrm{O}$, and the cellulose that certain sclerenchymatous and bast-tissues yielded to Koroll was of similar composition. The latter chemist also prepared it from parenchymatous tissues, and then it generally possessed a composition approximately indicated by the formula $5\left(\mathrm{C}_{6} \mathrm{H}_{10} \mathrm{O}_{5}\right)+2 \mathrm{H}_{2} \mathrm{O}$, whereas the wood of most dicotyledons contains, according to Stackmann, a cellulose of the formula $5\left(\mathrm{C}_{6} \mathrm{H}_{10} \mathrm{O}_{5}\right)+3 \mathrm{H}_{2} \mathrm{O}$. In these experiments the substance was exhausted with water, alcohol, dilute soda, dilute acid, a mixture of one part of sulphuric acid with four of water, and chlorinewater, previously to being treated with nitric acid and chlorate of potassium. Schuppe has shown that the action of the sulphuric acid, the use of which I recommend to be discontinued, results in the formation of a hydro-cellulose. If the treatment with sulphuric acid was omitted, the cellulose obtained from woods corresponded in composition to the formula $\mathrm{C}_{6} \mathrm{H}_{10} \mathrm{O}_{5}$. But the cellulose isolated from apples by a process that did not include treatment with sulphuric acid showed a deviation in composition from the formula $\mathrm{C}_{6} \mathrm{H}_{10} \mathrm{O}_{5}{ }^{2}$

The cellulose of fungi (cf. $\$ 249$ ) frequently shows a composition corresponding almost exactly to the formula $\mathrm{C}_{6} \mathrm{H}_{10} \mathrm{O}_{5}$.

$\S 249$. Varieties of Cellulose. - The variations observed in celluloses isolated from different plants is partly to be ascribed to the abovementioned difference in composition, and partly probably to variations in density. For instance, the cellulose of most phanerogams

1 Annal. d. Chem, und Pharm. cxxxviii. 1, 1866, and Jahresb. f. Pharm. 9, 1867. Compare also Bente, Ber. d. d. chem. Ges. xiii. 476, 1875; Journ. f. Landwirthsch. 166, 1876. Compare also Bevan and Cross on the chemistry of Bastfibre, Chem. News, xlii. 77, 91, 1880.

2 Compare the dissertations of Pfeil and Treffner already quoted. 
is dissolved by ammonio-sulphate of copper, ${ }^{1}$ and reprecipitated in an amorphous condition by dilute acids; but that of many fungi is either insoluble or taken up to a slight extent only, and then with great difficulty. Concentrated sulphuric acid and syrupy solution of chloride of zinc render cellulose capable of assuming a blue colour with iodine $;^{2}$ but in some instances the reaction is found to fail, ${ }^{3}$ and Schulze's reagent for cellulose, which is not without its value as a micro-chemical reagent, cannot therefore in such cases be employed for colouring the cell-wall. The facility, too, with which cellulose can be converted into glucose varies. Masing observes that fungus-cellulose undergoes the change more easily than flax-fibre. ${ }^{4}$

$\S 250$. Crude Fibre.-From what has been said of the isolation of cellulose, it follows that the crude fibre of the physiologist and agricultural chemist cannot be exactly identical with that substance. To estimate the crude fibre, the material is generally boiled for half an hour, first with 1 per cent. sulphuric acid, and then with 1 per cent. caustic potash. The residue is exhausted with cold water, alcohol, and ether in succession, dried and weighed. In this crude fibre we may anticipate the presence of a little undecomposed wood-gum, lignin, and suberin, as well as part of the hydrocelluloses mentioned in $\$ \S 117,244$.

An apparatus that may be used with advantage in this determination has been described by Holdefleiss. ${ }^{5}$

1 I prepare this reagent by precipitating hydrate of copper from a solution of the sulphate by dilute caustic soda, rapidly filtering off, pressing and dissolving in the requisite quantity of 20 per cent. solution of ammonia.

2 The reagent known as Schulze's can be prepared by dissolving 25 parts of dry chloride of zinc and 8 of iodide of potassium in $8 \frac{1}{2}$ of water, and adding as much iodine as the solution will take up when warmed for a short time with it.

${ }^{3}$ On cellulose of fungi, see Masing, Pharm. Zeitschr. f. Russland, ix. 385, 1870. Richter (Chem. Centralblatt, 483, 1881) has recently denied the existence of a special fungus-cellulose as the prolonged action of caustic alkalies converts it into ordinary cellulose. But is it not probable that such treatment actually produces a chemical change?

${ }^{4}$ On cellulose see Pajen, Annal. d. Sciences naturelles, xi. 21, xiv. 88; Fromberg, Annal. d. Chem. und Pharm. lii. 113; Heldt and Rochleder, ibid. xlviii. 8; Schlossberger and Döpping, ibid. lii. 106; Schlossberger, ibid. cvii. 24, 1858; Péligot, Comptes rendus, lxiii. 209, 1861 ; Knop and Schnedermann, Journ. f. prakt. Chem. xxxix. 363, xl. 389 ; Henneberg, Annal. d. Chem. und Pharm. cxlvi. 130, 1869 ; König, Zeitschr. f. anal. Chem. xiii. 242, $1 S 79$.

${ }^{5}$ Compare Holdefleiss, Zeitschr. f. Anal. Chem. xvi. 498, 1877, and Landwirthsch. Jahrb. Supp. vi. 101. 
PERCENTAGE COMPOSITION OF THE CONSTITUENTS OF PLANTS MENTIONED IN THE FOREGOING WORK.

\begin{tabular}{|c|c|c|c|c|c|c|c|c|}
\hline \multicolumn{3}{|c|}{ Name. } & Formula. & C. & H. & O. & N. & S. \\
\hline Abietic acid & $\bullet$ & • & $\mathrm{C}_{44} \mathrm{H}_{64} \mathrm{O}_{5}$ & $78 \cdot 57$ & $9 \cdot 52$ & $11 \cdot 91$ & & \\
\hline Absinthiin & - & - & $\mathrm{C}_{40} \mathrm{H}_{58} \mathrm{O}_{9}$ & $70 \cdot 38$ & $8 \cdot 50$ & $21 \cdot 12$ & & \\
\hline Acetic acid & - & - & $\mathrm{C}_{2} \mathrm{H}_{4} \mathrm{O}_{2}$ & $40 \cdot 00$ & $6 \cdot 66$ & $53 \cdot 33$ & & \\
\hline Achilleïne & - & - & $\mathrm{C}_{20} \mathrm{H}_{38} \mathrm{~N}_{2} \mathrm{O}_{15}$ & $43 \cdot 84$ & $6 \cdot 96$ & $43 \cdot 84$ & $5 \cdot 12$ & \\
\hline Aconitine & 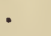 & - & $\mathrm{C}_{33} \mathrm{H}_{43} \mathrm{NO}_{12}$ & $61 \cdot 39$ & $6 \cdot 67$ & $29 \cdot 77$ & $2 \cdot 17$ & \\
\hline Aconitic acid & & - & $\mathrm{C}_{6} \mathrm{H}_{6} \mathrm{O}_{6}$ & $41 \cdot 38$ & $3 \cdot 45$ & $55 \cdot 17$ & & \\
\hline Adansonin & - & - & $\mathrm{C}_{48} \mathrm{H}_{72} \mathrm{O}_{33}$ & $48 \cdot 30$ & $5 \cdot 95$ & $45 \cdot 75$ & & \\
\hline Aesculin & - & - & $\mathrm{C}_{21} \mathrm{H}_{24} \mathrm{O}_{13}$ & $52 \cdot 07$ & $4 \cdot 96$ & $42 \cdot 97$ & & \\
\hline Albumin & - & & $?$ & $\begin{array}{l}52 \cdot 45- \\
53 \cdot 97\end{array}$ & $\begin{array}{l}6 \cdot 81- \\
7 \cdot 77\end{array}$ & $\begin{array}{l}22 \cdot 21- \\
23 \cdot 50\end{array}$ & $\begin{array}{l}15 \cdot 65- \\
15 \cdot 92\end{array}$ & 0.8 \\
\hline Alizarin & - & - & $\mathrm{C}_{14} \mathrm{H}_{8} \mathrm{O}_{3}$ & $75 \cdot 00$ & $3 \cdot 57$ & $21 \cdot 44$ & & \\
\hline Alkannin & - & 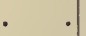 & $\mathrm{C}_{15} \mathrm{H}_{14}^{\circ} \mathrm{O}_{4}^{\circ}$ & $69 \cdot 72$ & $5 \cdot 42$ & $24 \cdot 86$ & & \\
\hline Amanitine & - & • & $\mathrm{C}_{5} \mathrm{H}_{14} \mathrm{NO}$ & $57 \cdot 69$ & $13 \cdot 46$ & $15 \cdot 38$ & $13 \cdot 46$ & \\
\hline Amygdalin & - & • & $\mathrm{C}_{20} \mathrm{H}_{27} \mathrm{NO}_{11}$ & $52 \cdot 51$ & $5 \cdot 91$ & $38 \cdot 52$ & 3.06 & \\
\hline Amyrin & & • & $\mathrm{C}_{25} \mathrm{H}_{42} \mathrm{O}$ & $83 \cdot 49$ & $11 \cdot 79$ & $4 \cdot 73$ & & \\
\hline Anacardic aci & & - & $\mathrm{C}_{44} \mathrm{H}_{64} \mathrm{O}_{7}$ (?) & $75 \cdot 04$ & 9.07 & $15 \cdot 89$ & & \\
\hline Anemonin & - & • & $\mathrm{C}_{15} \mathrm{H}_{12} \mathrm{O}_{6}$ (?) & $62 \cdot 50$ & $4 \cdot 17$ & $33 \cdot 33$ & & \\
\hline Anethol. & - & - & $\mathrm{C}_{10} \mathrm{H}_{12} \mathrm{O}$ & $81 \cdot 08$ & $8 \cdot 11$ & $10 \cdot 81$ & & \\
\hline Angelic acid & . & • & $\mathrm{C}_{5} \mathrm{H}_{8} \mathrm{O}_{2}$ & 60.00 & $8 \cdot 00$ & $32 \cdot 00$ & & \\
\hline Antiarin & - & . & $\mathrm{C}_{14} \mathrm{H}_{20} \mathrm{O}_{5}$ & $62 \cdot 68$ & $7 \cdot 46$ & $29 \cdot 85$ & & \\
\hline Apiin & - & • & $\mathrm{C}_{27} \mathrm{H}_{32} \mathrm{O}_{16}$ & $52 \cdot 9$ & $5 \cdot 2$ & $41 \cdot 9$ & & \\
\hline Arabic acid & . & - & $\mathrm{C}_{12} \mathrm{H}_{22} \mathrm{O}_{11}$ & $42 \cdot 10$ & $6 \cdot 43$ & $51 \cdot 47$ & & \\
\hline Arachic acid & . & . & $\mathrm{C}_{20} \mathrm{H}_{40} \mathrm{O}_{2}$ & $76 \cdot 92$ & $12 \cdot 82$ & $10 \cdot 26$ & & \\
\hline Arbutin & - & . & $\mathrm{C}_{25} \mathrm{H}_{34} \mathrm{O}_{14}$ & $53 \cdot 7$ & $6 \cdot 1$ & $40 \cdot 2$ & & \\
\hline Aribine & - & • & $\mathrm{C}_{23} \mathrm{H}_{20} \mathrm{~N}_{4}$ & $78 \cdot 43$ & $5 \cdot 68$ & & $15 \cdot 89$ & \\
\hline Aricine & - & . & $\mathrm{C}_{23} \mathrm{H}_{26} \mathrm{~N}_{2} \hat{O}_{4}$ & $70 \cdot 05$ & $6 \cdot 59$ & $16 \cdot 25$ & $7 \cdot 11$ & \\
\hline Asclepin & - & - & $\mathrm{C}_{20} \mathrm{H}_{34} \mathrm{O}_{3}$ & $74 \cdot 54$ & $10 \cdot 56$ & $14 \cdot 90$ & & \\
\hline Asparagin & & & $\mathrm{C}_{4} \mathrm{H}_{8} \mathrm{~N}_{2} \mathrm{O}_{3}$ & $36 \cdot 36$ & $6 \cdot 06$ & $36 \cdot 37$ & $21 \cdot 21$ & \\
\hline Aspidospermi & & - & $\mathrm{C}_{22} \mathrm{H}_{30} \mathrm{~N}_{2} \mathrm{O}_{2}$ & $74 \cdot 57$ & $8 \cdot 48$ & $9 \cdot 04$ & $7 \cdot 91$ & \\
\hline Athamantin & & - & $\mathrm{C}_{24} \mathrm{H}_{30} \mathrm{U}_{7}$ & $66 \cdot 98$ & $6 \cdot 98$ & $26 \cdot 04$ & & \\
\hline Atherospermi & ine & - & $\mathrm{C}_{30} \mathrm{H}_{40} \mathrm{~N}_{2} \mathrm{O}_{5}$ & $70 \cdot 87$ & $7 \cdot 87$ & $15 \cdot 75$ & $5 \cdot 51$ & \\
\hline Atropine & - & - & $\mathrm{C}_{17} \mathrm{H}_{23} \mathrm{NO}_{3}$ & 70.58 & $7 \cdot 95$ & $16 \cdot 60$ & $4 \cdot 84$ & \\
\hline Barbaloïn & - & - & $\mathrm{C}_{17} \mathrm{H}_{20} \mathrm{O}_{7}$ (?) & $60 \cdot 71$ & $5 \cdot 95$ & $33 \cdot 34$ & & \\
\hline Bassorin & . & - & $\mathrm{C}_{12} \mathrm{H}_{22} \mathrm{O}_{11}$ & $42 \cdot 10$ & $6 \cdot 43$ & $51 \cdot 47$ & & \\
\hline Beberine & 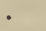 & - & $\mathrm{C}_{19} \mathrm{H}_{21} \mathrm{NO}_{3}$ & $73 \cdot 31$ & $6 \cdot 75$ & $15 \cdot 44$ & $4 \cdot 50$ & \\
\hline Benzaldehyde & & . & $\mathrm{C}_{7} \mathrm{H}_{6} \mathrm{O}$ & $79 \cdot 24$ & $5 \cdot 65$ & $15 \cdot 11$ & & \\
\hline Benzoic acid & . & . & $\mathrm{C}_{7} \mathrm{H}_{6} \mathrm{O}_{2}$ & $68 \cdot 85$ & $4 \cdot 92$ & $26 \cdot 23$ & & \\
\hline Benzohelicin & . & . & $\mathrm{C}_{20} \mathrm{H}_{20} \mathrm{O}_{8}$ & $61 \cdot 86$ & $5 \cdot 15$ & 32.99 & & \\
\hline Berberine & . & • & $\mathrm{C}_{20} \mathrm{H}_{17} \mathrm{NO}_{4}$ & $71 \cdot 64$ & $5 \cdot 08$ & $19 \cdot 10$ & $4 \cdot 18$ & \\
\hline Betaïne. & - & • & $\mathrm{C}_{5} \mathrm{H}_{13} \mathrm{NO}_{3}$ & $44 \cdot 44$ & $9 \cdot 63$ & $35 \cdot 55$ & $10 \cdot 37$ & \\
\hline Betaorcin & . & . & $\mathrm{C}_{8} \mathrm{H}_{10} \mathrm{O}_{2}$ & $69 \cdot 56$ & $7 \cdot 24$ & $23 \cdot 20$ & & \\
\hline Betulin . & . & • & $\mathrm{C}_{36} \mathrm{H}_{60} \mathrm{O}_{2}$ & $82 \cdot 57$ & $11 \cdot 36$ & $6 \cdot 06$ & & \\
\hline Bixine . & - & . & $\mathrm{C}_{28} \mathrm{H}_{34} \mathrm{O}_{5}$ & $74 \cdot 66$ & $7 \cdot 55$ & $17 \cdot 78$ & & \\
\hline Boheic acid & . & • & $\mathrm{C}_{7} \mathrm{H}_{10} \mathrm{O}_{6}$ & $44 \cdot 21$ & $5 \cdot 26$ & $50 \cdot 53$ & & \\
\hline Borneol & . & - & $\mathrm{C}_{10} \mathrm{H}_{18} \mathrm{O}$ & $77 \cdot 92$ & $11 \cdot 69$ & $10 \cdot 39$ & & \\
\hline Brasillin & - & - & $\mathrm{C}_{16} \mathrm{H}_{14} \mathrm{O}_{5}$ & $67 \cdot 11$ & $5 \cdot 43$ & $27 \cdot 46$ & & \\
\hline Brucine & - & 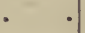 & $\mathrm{C}_{23} \mathrm{H}_{26} \mathrm{~N}_{2} \mathrm{O}_{4}$ & 70.00 & $6 \cdot 64$ & $16 \cdot 26$ & $7 \cdot 10$ & \\
\hline Bryonin & $\cdot$ & • & $\mathrm{C}_{48} \mathrm{H}_{80} \mathrm{O}_{19}$ & $60 \cdot 00$ & $8 \cdot 33$ & $31 \cdot 66$ & & \\
\hline Bryoïdin & . & & $\mathrm{C}_{20} \mathrm{H}_{33} \mathrm{O}_{3}$ & $73 \cdot 62$ & $11 \cdot 66$ & $14 \cdot 72$ & & \\
\hline
\end{tabular}




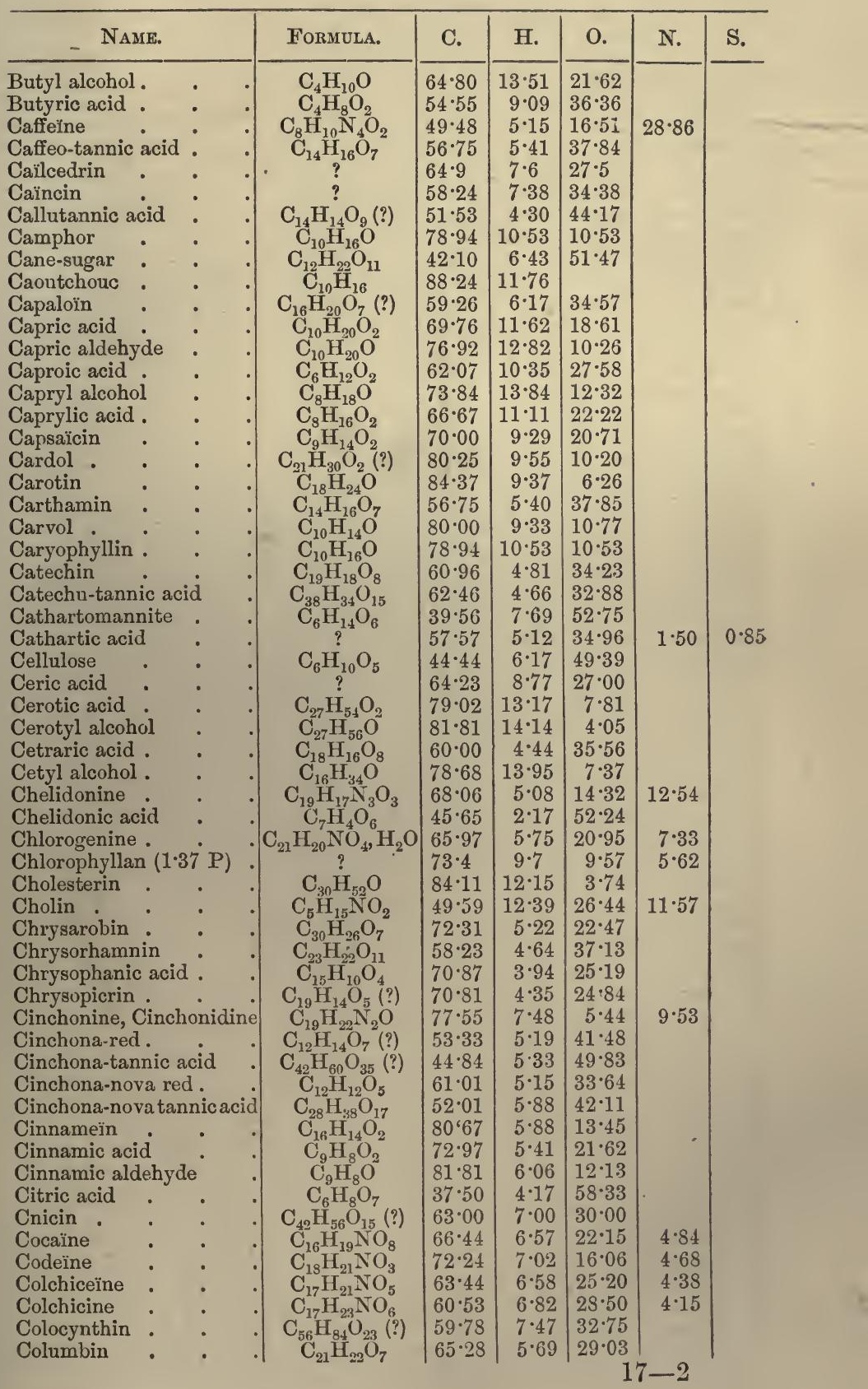




\begin{tabular}{|c|c|c|c|c|c|c|c|c|}
\hline \multicolumn{3}{|c|}{ NAME. } & Formula. & C. & H. & O. & N. & S. \\
\hline \multicolumn{3}{|c|}{ Conessine (Wrightine) } & $?$ & $78 \cdot 3$ & $11 \cdot 2$ & 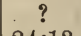 & & \\
\hline Conglutin & - & ${ }^{\circ}$ & $?$ & $50 \cdot 24$ & $6 \cdot 81$ & $24 \cdot 13$ & $18 \cdot 37$ & 0.45 \\
\hline Conhydrine & - & . & $\mathrm{C}_{8} \mathrm{H}_{17} \mathrm{NO}$ & $67 \cdot 12$ & $11 \cdot 89$ & $11 \cdot 19$ & $9 \cdot 79$ & \\
\hline Coniferin & - & . & $\mathrm{C}_{16} \mathrm{H}_{22} \mathrm{O}_{8}$ & $56 \cdot 14$ & $6 \cdot 43$ & $37 \cdot 43$ & & \\
\hline Coniine . & & . & $\mathrm{C}_{8} \mathrm{H}_{15} \mathrm{~N}$ & $76 \cdot 81$ & $12 \cdot 00$ & & $11 \cdot 20$ & \\
\hline Convallamari & & - & $\mathrm{C}_{23} \mathrm{H}_{44} \mathrm{O}_{12}$ & $53 \cdot 91$ & $8 \cdot 59$ & $37 \cdot 50$ & & \\
\hline Convallarin & - & - & $\mathrm{C}_{34} \mathrm{H}_{62} \mathrm{O}_{11}$ & $\begin{array}{l}63 \cdot 16 \\
51 \cdot 87\end{array}$ & $\begin{array}{l}9 \cdot 60 \\
7 \cdot 37\end{array}$ & $27 \cdot 24$ & & \\
\hline $\begin{array}{l}\text { Convolvulin } \\
\text { Coriamyrtin }\end{array}$ & : & : & $\mathrm{C}_{31} \mathrm{H}_{50} \mathrm{O}_{16}$ & $\begin{array}{l}54 \cdot 87 \\
63 \cdot 86\end{array}$ & $\begin{array}{l}7.37 \\
6.38\end{array}$ & $\begin{array}{l}37 \cdot 76 \\
29 \cdot 76\end{array}$ & & \\
\hline $\begin{array}{l}\text { Coriamyrtin } \\
\text { Cotoïn . }\end{array}$ & & $\dot{.}$ & $\mathrm{C}_{22}^{20} \mathrm{H}_{18}^{24} \mathrm{O}_{6}$ & $69 \cdot 84$ & $4 \cdot 76$ & $25 \cdot 39$. & & \\
\hline $\begin{array}{l}\text { Cotoïn } \cdot \\
\text { Crocin : }\end{array}$ & & & $\mathrm{C}_{48} \mathrm{H}_{60} \mathrm{O}_{18}$ & $62 \cdot 33$ & $6 \cdot 49$ & $31 \cdot 17$ & & \\
\hline Crotonic acid & & - & $\mathrm{C}_{4} \mathrm{H}_{6} \mathrm{O}_{2}$ & $55 \cdot 81$ & $6 \cdot 99$ & $37 \cdot 20$ & & \\
\hline Cubebin & - & - & $\mathrm{C}_{10} \mathrm{H}_{10} \mathrm{O}_{3}$ & $67 \cdot 42$ & $5 \cdot 62$ & 26.96 & & \\
\hline Cumarin & $\cdot$ & $\cdot$ & $\mathrm{C}_{9} \mathrm{H}_{6} \mathrm{O}_{2}$ & $73 \cdot 97$ & $4 \cdot 11$ & $21 \cdot 92$ & & \\
\hline Curaçao-aloïn & & $\cdot$ & $\mathrm{C}_{15} \mathrm{H}_{17} \mathrm{O}_{7}$ & $58 \cdot 22$ & $5 \cdot 50$ & $36 \cdot 28$ & & \\
\hline Curarine & • & - & $\mathrm{C}_{18} \mathrm{H}_{35} \mathrm{~N}$ (?) & $81 \cdot 51$ & $13 \cdot 21$ & & $5 \cdot 28$ & \\
\hline Curcumin & - & - & $\mathrm{C}_{10} \mathrm{H}_{10} \mathrm{O}_{3}$ & $67 \cdot 41$ & $5 \cdot 62$ & $26 \cdot 96$ & & \\
\hline Cusconine & - & . & $\mathrm{C}_{23} \mathrm{H}_{26} \mathrm{~N}_{2} \mathrm{O}_{4}$ & $70^{\circ} 05$ & $6 \cdot 59$ & $16 \cdot 25$ & $7 \cdot 11$ & \\
\hline Cyclamin & - & . & $\mathrm{C}_{20} \mathrm{H}_{34} \mathrm{O}_{10}$ & $55 \cdot 29$ & $7 \cdot 83$ & 36.87 & & \\
\hline Cyclopin & - & . & $\mathrm{C}_{14} \mathrm{H}_{18} \mathrm{O}_{12}$ & $44 \cdot 44$ & $4 \cdot 76$ & $51 \cdot 80$ & & \\
\hline Cytisine & - & ${ }^{\circ}$ & $\mathrm{C}_{20} \mathrm{H}_{27} \mathrm{~N}_{3} \mathrm{O}$ & $73 \cdot 85$ & $8 \cdot 31$ & $4 \cdot 92$ & $12 \cdot 92$ & \\
\hline Daphnin & - & - & $\mathrm{C}_{31} \mathrm{H}_{34} \mathrm{O}_{19}$ & $52 \cdot 39$ & $4 \cdot 78$ & 42.83 & & \\
\hline Datiscin & - & . & $\mathrm{C}_{21} \mathrm{H}_{22} \mathrm{O}_{12}$ & $54 \cdot 08$ & $4 \cdot 72$ & $41 \cdot 20$ & & \\
\hline Delphinine & - & . & $\mathrm{C}_{22} \mathrm{H}_{35} \mathrm{NO}_{6}$ & $64 \cdot 55$ & $8 \cdot 66$ & $23 \cdot 47$ & $3 \cdot 42$ & \\
\hline Delphinoïdine & & - & $\mathrm{C}_{42} \mathrm{H}_{68} \mathrm{~N}_{2} \mathrm{O}_{7}$ & $70 \cdot 9$ & $9 \cdot 5$ & $15 \cdot 6$ & $3 \cdot 9$ & \\
\hline Dextrin . & . & . & $\overline{\mathrm{C}}_{6} \mathrm{H}_{10} \mathrm{O}_{5}$ & $44 \cdot 44$ & $6 \cdot 17$ & $49 \cdot 39$ & & \\
\hline Digitalin & - & ${ }^{\circ}$ & $\mathrm{C}_{5} \mathrm{H}_{8} \mathrm{O}_{2}(?)$ & $59 \cdot 95$ & 8.05 & $32 \cdot 00$ & & \\
\hline Digitonin & . & . & $\mathrm{C}_{31} \mathrm{H}_{52} \mathrm{O}_{17}$ & $53 \cdot 21$ & $7 \cdot 60$ & $39 \cdot 19$ & & \\
\hline Digitoxin & - & . & $\mathrm{C}_{21} \mathrm{H}_{32} \mathrm{O}_{7}$ & $63 \cdot 60$ & $8 \cdot 50$ & $27 \cdot 90$ & & \\
\hline Ditaïne . & - & . & $\mathrm{C}_{22} \mathrm{H}_{30} \mathrm{~N}_{2} \mathrm{O}_{4}$ & $68 \cdot 39$ & $7 \cdot 77$ & 16.58 & $7 \cdot 25$ & \\
\hline Dulcamarin & $\cdot$ & $\cdot$ & $\mathrm{C}_{22} \mathrm{H}_{34} \mathrm{O}_{10}$ & $57 \cdot 64$ & $7 \cdot 42$ & $34 \cdot 94$ & & \\
\hline Dulcite . & - & - & $\mathrm{C}_{6} \mathrm{H}_{14} \mathrm{O}_{6}$ & $39 \cdot 56$ & $7 \cdot 69$ & $52 \cdot 75$ & & \\
\hline Elaterin & & - & $\mathrm{C}_{20} \mathrm{H}_{28} \mathrm{O}_{5}$ & $68 \cdot 96$ & $8 \cdot 04$ & $23 \cdot 00$ & & \\
\hline Ellago-tannic & acid & & $\mathrm{C}_{14} \mathrm{H}_{10} \mathrm{O}_{10}$ & $49 \cdot 69$ & $3 \cdot 16$ & $47 \cdot 25$ & & \\
\hline Ellagic acid & - & ${ }^{\circ}$ & $\mathrm{C}_{14} \mathrm{H}_{6} \mathrm{O}_{8}$ & $55 \cdot 63$ & $1 \cdot 99$ & $42 \cdot 38$ & & \\
\hline Eraodin. & - & . & $\mathrm{C}_{15} \mathrm{H}_{10} \mathrm{O}_{5}$ & $66 \cdot 67$ & $3 \cdot 70$ & $30 \cdot 63$ & & \\
\hline Emulsin & - & . & & $48 \cdot 78$ & $7 \cdot 73$ & $24 \cdot 67$ & $18 \cdot 82$ & ? \\
\hline Ericolin & - & - & $\mathrm{C}_{34} \mathrm{H}_{56} \mathrm{O}_{21}$ & $51 \cdot 00$ & $7 \cdot 00$ & $42 \cdot 00$ & & \\
\hline Erythrite & & . & $\mathrm{C}_{4} \mathrm{H}_{10} \mathrm{O}_{4}^{-}$ & $39 \cdot 34$ & $8 \cdot 20$ & $52 \cdot 46$ & & \\
\hline Erythrocental & urin & . & $\mathrm{C}_{27} \mathrm{H}_{24} \mathrm{O}_{8}$ & $68 \cdot 07$ & $5 \cdot 04$ & $26 \cdot 89$ & & \\
\hline Ethyl alcohol & & . & $\mathrm{C}_{2} \mathrm{H}_{6} \mathrm{O}$ & $52 \cdot 17$ & 13.04 & $34 \cdot 79$ & & \\
\hline Eugenin & . & . & $\mathrm{C}_{10} \mathrm{H}_{12} \mathrm{O}_{2}$ & $73 \cdot 17$ & $7 \cdot 32$ & 19.51 & & \\
\hline Eugenol & $\cdot$ & 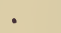 & $\mathrm{C}_{10} \mathrm{H}_{12} \mathrm{O}_{2}$ & $73 \cdot 17$ & $7 \cdot 32$ & $19 \cdot 51$ & & \\
\hline Euphorbon & . & - & $\mathrm{C}_{15} \mathrm{H}_{24} \mathrm{O}$ & $81 \cdot 82$ & $11 \cdot 04$ & $7 \cdot 14$ & & \\
\hline Evernic acid & & $\theta^{\circ}$ & $\mathrm{C}_{17} \mathrm{H}_{16} \mathrm{O}_{7}$ & $61^{\circ} 44$ & $4 \cdot 82$ & $33 \cdot 74$ & & \\
\hline Everninic acic & & . & $\mathrm{C}_{9} \mathrm{H}_{10} \mathrm{O}_{4}$ & $59 \cdot 34$ & $5 \cdot 49$ & $35 \cdot 17$ & & \\
\hline Ferulic acid & 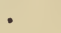 & - & $\mathrm{C}_{10} \mathrm{H}_{10} \mathrm{O}_{4}$ & $61 \cdot 23$ & $6 \cdot 12$ & $32 \cdot 65$ & & \\
\hline Filicin & & - & $\mathrm{C}_{26} \mathrm{H}_{30} \mathrm{O}_{9}$ & $64 \cdot 20$ & $6 \cdot 17$ & $29 \cdot 63$ & & \\
\hline Frangulic acic & & $\cdot$ & $\mathrm{C}_{16} \mathrm{H}_{12} \mathrm{O}_{5}$ & $67 \cdot 6$ & $4 \cdot 2$ & $38 \cdot 2$ & & \\
\hline Fraxin & . & 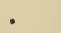 & $\mathrm{C}_{54} \mathrm{H}_{62} \mathrm{O}_{35}$ & $51 \cdot 02$ & $4 \cdot 89$ & $44 \cdot 89$ & & \\
\hline Fruit-sugar & & & $\mathrm{C}_{6} \mathrm{H}_{12} \mathrm{O}_{6}$ & .40 .00 & $6 \cdot 66$ & $53 \cdot 33$ & & \\
\hline Fumaric acid & & ${ }^{\circ}$ & $\mathrm{C}_{4} \mathrm{H}_{4} \mathrm{O}_{4}$ & $41 \cdot 38$ & $3 \cdot 45$ & $55 \cdot 17$ & & \\
\hline Galactose & & & $\mathrm{C}_{6} \mathrm{H}_{12} \mathrm{O}_{6}$ & $40 \cdot 00$ & $6 \cdot 66$ & $53 \cdot 33$ & & \\
\hline Galitannic aci & & 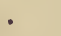 & $\mathrm{C}_{7} \mathrm{H}_{8} \mathrm{O}_{5}$ (?) & $48 \cdot 84$ & $4 \cdot 65$ & $46 \cdot 51$ & & \\
\hline Gallic acid & 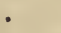 & 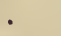 & $\mathrm{C}_{7} \mathrm{H}_{6} \mathrm{O}_{5}$ & $49 \cdot 49$ & $3 \cdot 65$ & $46 \cdot 86$ & & \\
\hline Gardenin & ${ }^{\circ}$ & • & $\mathrm{C}_{14} \mathrm{H}_{12} \mathrm{O}_{6}$ & $60 \cdot 85$ & $4 \cdot 75$ & $34 \cdot 40$ & & \\
\hline Gelsemine & 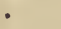 & - & $\mathrm{C}_{11} \mathrm{H}_{19} \mathrm{NO}_{2}$ & $67 \cdot 00$ & $9 \cdot 64$ & $16 \cdot 30$ & $7 \cdot 10$ & \\
\hline Gentisin & & . & $\mathrm{C}_{14} \mathrm{H}_{10} \mathrm{O}_{5}$ & $65 \cdot 11$ & $3 \cdot 87$ & $31 \cdot 02$ & & \\
\hline
\end{tabular}




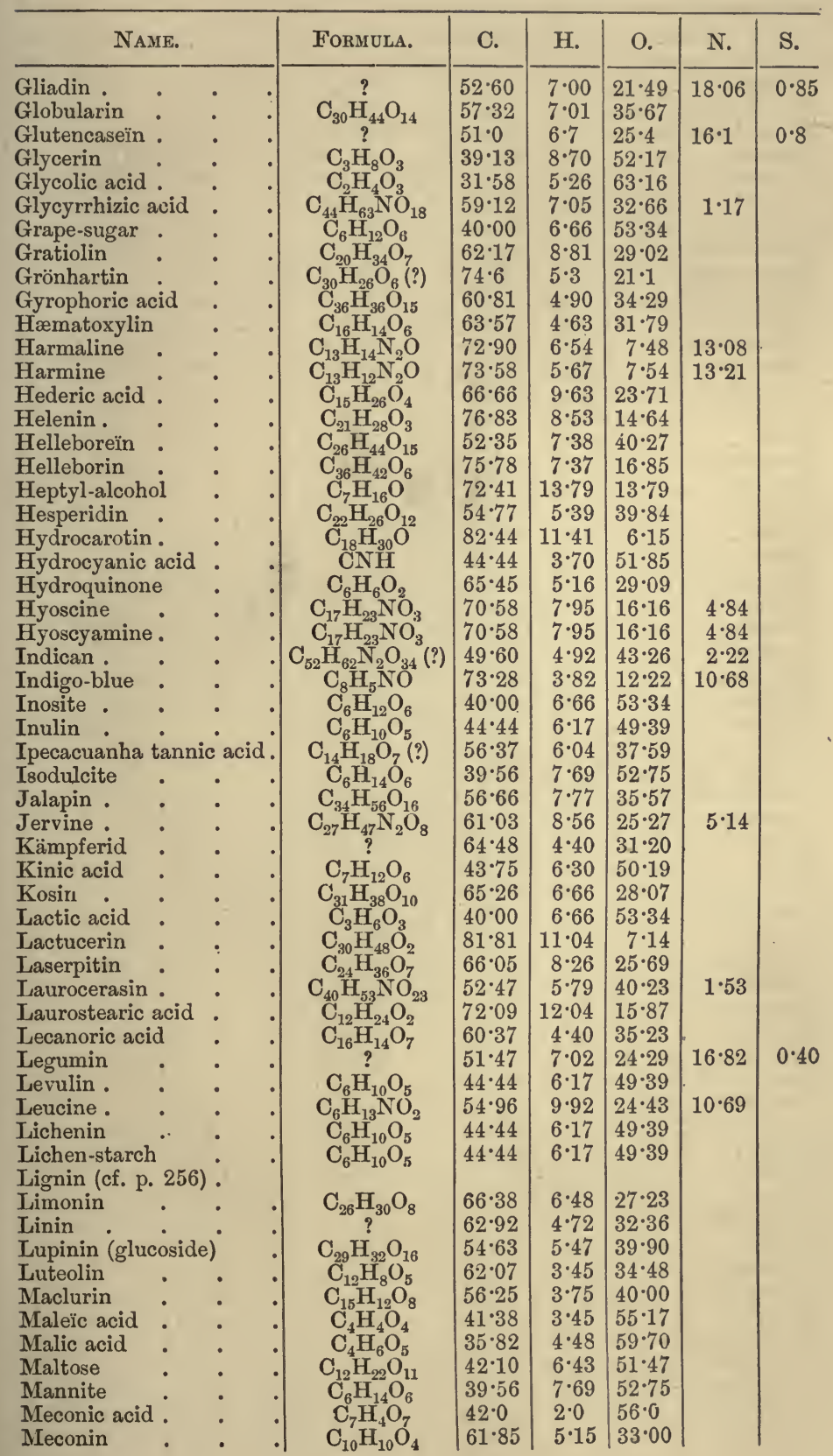




\begin{tabular}{|c|c|c|c|c|c|c|c|c|}
\hline \multicolumn{3}{|c|}{ NAME. } & Formula. & C. & H. & O. & N. & $\mathrm{S}$. \\
\hline Melanthin & $\cdot$ & & & $62 \cdot 4$ & $9 \cdot 0$ & $28 \cdot 6$ & & \\
\hline Melezitose & & - & & $42 \cdot 10$ & $6 \cdot 43$ & $51 \cdot 47$ & & \\
\hline Melissyl alcol & hol & - & & $82 \cdot 19$ & $14 \cdot 15$ & $3 \cdot 66$ & & \\
\hline Melitose & & - & & $42 \cdot 10$ & $6 \cdot 43$ & $51 \cdot 47$ & & \\
\hline Menispermin & & . & & $72 \cdot 00$ & $8 \cdot 00$ & $10 \cdot 65$ & $9 \cdot 35$ & \\
\hline Menthol & & - & & 76.93 & $12 \cdot 82$ & $10 \cdot 25$ & & \\
\hline Menyanthin & & • & & $55 \cdot 46$ & $7 \cdot 56$ & $36 \cdot 98$ & & \\
\hline Metarabic ac & & • & $\mathrm{C}_{12}^{22} \mathrm{H}_{22} \mathrm{O}_{11}$ & $42 \cdot 10$ & $6 \cdot 43$ & $51 \cdot 47$ & & \\
\hline Methyl alcoh & & . & & $37 \cdot 50$ & $12 \cdot 50$ & 50.00 & & \\
\hline Methylamine & & - & & $38 \cdot 71$ & $16 \cdot 13$ & $\ldots$ & $45 \cdot 17$ & \\
\hline Methylconiin & & . & $\mathrm{C}_{9} \mathrm{H}_{17} \mathrm{~N}$ & $77 \cdot 69$ & $12 \cdot 23$ & & 10.07 & \\
\hline Methysticin & 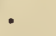 & • & & $65 \cdot 85$ & $5 \cdot 64$ & $28 \cdot 51$ & & \\
\hline Milk-sugar & & - & & $42 \cdot 10$ & $6 \cdot 43$ & $51 \cdot 47$ & & \\
\hline Mongumic ac & & - & & $66 \cdot 0$ & $4 \cdot 6$ & $29 \cdot 3$ & & \\
\hline Morphine & - & . & $\mathrm{VO}_{3}$ & $71 \cdot 58$ & $6 \cdot 67$ & $16 \cdot 84$ & $4 \cdot 91$ & \\
\hline Morin : & - & - & & $59 \cdot 61$ & $3 \cdot 31$ & $37 \cdot 08$ & & \\
\hline Moschatine & - & - & & $68 \cdot 22$ & $6 \cdot 66$ & $27 \cdot 65$ & $3 \cdot 45$ & \\
\hline Mucedin & - & - & & $54 \cdot 11$ & 6.90 & $21 \cdot 48$ & $16 \cdot 63$ & 0.88 \\
\hline Muscarine & . & . & $\mathrm{NO}_{2}$ & $50 \cdot 42$ & $10 \cdot 92$ & $26 \cdot 89$ & $11 \cdot 77$ & \\
\hline Mycose & & & & $42 \cdot 10$ & $6 \cdot 43$ & $51 \cdot 47$ & & \\
\hline Myristic acid & & & & $73 \cdot 68$ & $12 \cdot 28$ & $14 \cdot 04$ & & \\
\hline Myronic acid & & - & $\mathrm{C}_{10} \mathrm{H}_{19} \mathrm{NS}_{2} \mathrm{O}_{10}$ & $31 \cdot 83$ & $5 \cdot 04$ & $42 \cdot 42$ & $3 \cdot 72$ & 16.99 \\
\hline Narcëine & - & . & $\overline{\mathrm{C}}_{23} \mathrm{H}_{29} \mathrm{NOO}_{9}$ & $59 \cdot 63$ & $6 \cdot 28$ & $31 \cdot 09$ & $3 \cdot 02$ & \\
\hline Narcotine & - & - & $\mathrm{C}_{22} \mathrm{H}_{23} \mathrm{NO}_{7}$ & $63 \cdot 92$ & $5 \cdot 57$ & $27 \cdot 12$ & $3 \cdot 39$ & \\
\hline Naringin & - & . & & $55 \cdot 6$ & $5 \cdot 6$ & 38.8 & & \\
\hline Nataloïn & - & 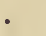 & $7(?)$ & $59 \cdot 44$ & $5 \cdot 88$ & $34^{\circ} 68$ & & \\
\hline Nepaline & . & & $\mathrm{O}_{12}$ & $63 \cdot 09$ & $7 \cdot 47$ & $27 \cdot 32$ & $2 \cdot 12$ & \\
\hline Nicotine & - & - & $\mathrm{N}_{2}^{20}$ & $74 \cdot 08$ & $8 \cdot 64$ & & $17 \cdot 28$ & \\
\hline Nucite . & & & $\mathrm{H}_{12} \mathrm{O}_{6}$ & 40.00 & 6.66 & $53 \cdot 33$ & & \\
\hline Oak-bark tan & nic a & & $\mathrm{H}_{16} \mathrm{O}_{16}^{\circ}$ & 53.85 & $5 \cdot 13$ & $41 \cdot 02$ & & \\
\hline Oenanthic ac & & - & & $64 \cdot 12$ & $11 \cdot 44$ & $24 \cdot 44$ & & \\
\hline Oleic acid & - & - & $\mathrm{O}_{2}$ & $76 \cdot 59$ & $12 \cdot 06$ & $11 \cdot 35$ & & \\
\hline Ononin & - & 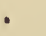 & ${ }_{34} \mathrm{O}_{13}$ & $59 \cdot 80$ & $5 \cdot 64$ & $34 \cdot 56$ & & \\
\hline Orcin & - & . & $\mathrm{O}_{2}$ & $67 \cdot 76$ & $6 \cdot 45$ & $25 \cdot 81$ & & \\
\hline Orsellic acid & . & . & $a^{2}$ & $57 \cdot 15$ & $4 \cdot 76$ & $38 \cdot 09$ & & \\
\hline Ostruthiin & - & - & $\mathrm{C}_{14} \stackrel{\mathrm{H}}{17}_{17} \mathrm{O}_{2}$ (?) & $77 \cdot 07$ & $7 \cdot 95$ & $14 \cdot 98$ & & \\
\hline Oxalic acid & . & - & & $26 \cdot 66$ & $2 \cdot 22$ & $71 \cdot 11$ & & \\
\hline Oxyacanthin & & & $20_{11}$ & $60 \cdot 57$ & $7 \cdot 26$ & $27 \cdot 76$ & $4 \cdot 42$ & \\
\hline Pæoniofluore & $\operatorname{scin}$ & . & $\mathrm{C}_{12} \mathrm{H}_{10} \mathrm{O}_{2}, \mathrm{H}_{2} \mathrm{O}$ & $71 \cdot 38$ & $5 \cdot 89$ & $24 \cdot 73$ & & \\
\hline Palmitic acid & & & $\mathrm{C}_{16} \mathrm{H}_{32} \mathrm{O}_{2}$ & $75 \cdot 00$ & $12 \cdot 50$ & $12 \cdot 50$ & & \\
\hline Papaverine & - & & ${ }_{21} \mathrm{NO}_{4}$ & $70 \cdot 79$ & $6 \cdot 20$ & $18 \cdot 88$ & $4 \cdot 13$ & \\
\hline Pararabin & - & 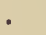 & ${ }_{22} \mathrm{O}_{11}$ & $42 \cdot 10$ & $6 \cdot 43$ & $51 \cdot 47$ & & \\
\hline Parellic actid & - & & $\mathrm{C}_{9} \mathrm{H}_{6} \mathrm{O}_{4}$ & $60 \cdot 67$ & $3 \cdot 37$ & $35 \cdot 96$ & & \\
\hline Paracotoïn & - & & $\mathrm{H}_{12} \mathrm{O}_{6}$ & $67 \cdot 85$ & $3 \cdot 57$ & $28 \cdot 58$ & & \\
\hline Paricine & - & & ${ }_{18} \mathrm{~N}_{2} \mathrm{O}$ & $75 \cdot 59$ & $7 \cdot 09$ & $6 \cdot 29$ & $11 \cdot 02$ & \\
\hline Paridin . & - & 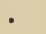 & $\mathrm{H}_{28} \mathrm{O}_{7}$ & $57 \cdot 83$ & $8 \cdot 43$ & $33 \cdot 74$ & & \\
\hline Parillin . & - & & ${ }_{24} \mathrm{O}_{2}$ & $60 \cdot 4$ & $9 \cdot 0$ & $30 \cdot 6$ & & \\
\hline Paytine. & • & & $\mathrm{N}_{2} \mathrm{O}$ & $79 \cdot 74$ & $6 \cdot 33$ & $5 \cdot 06$ & $8 \cdot 86$ & \\
\hline Peucedanin & - & & $\mathrm{C}_{12} \mathrm{H}_{12} \mathrm{O}_{3}$ & 70.58 & $5 \cdot 88$ & $23 \cdot 54$ & & \\
\hline Philyrin & - & 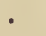 & $\mathrm{C}_{27} \mathrm{H}_{34} \mathrm{O}_{11}$ & $60 \cdot 67$ & $6 \cdot 37$ & $32 \cdot 96$ & & \\
\hline Phlorizin & $\cdot$ & & $\mathrm{C}_{21}^{-} \mathrm{H}_{24} \mathrm{O}_{10}$ & $56 \cdot 15$ & $5 \cdot 81$ & $38 \cdot 04$ & & \\
\hline Phloroglucin & & & $\mathrm{C}_{6} \mathrm{H}_{6} \mathrm{O}_{3}$ & $57 \cdot 13$ & $4 \cdot 76$ & $38 \cdot 11$ & & \\
\hline Physalin & & & $\mathrm{C}_{14} \mathrm{H}_{16} \mathrm{O}_{5}$ & $63 \cdot 64$ & $6 \cdot 06$ & $30 \cdot 30$ & & \\
\hline Physostigmin & & & & $65 \cdot 49$ & $7 \cdot 64$ & $11 \cdot 60$ & $15 \cdot 27$ & \\
\hline Phytosterin & & & & $83 \cdot 87$ & $11 \cdot 83$ & $4 \cdot 30$ & & \\
\hline Picropodophy & fllin & • & & $67 \cdot 71$ & $5 \cdot 88$ & $26 \cdot 41$ & & \\
\hline Picroroccellin & & 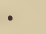 & $\mathrm{C}_{27} \mathrm{H}_{29} \mathrm{~N}_{3} \mathrm{O}_{5}$ & $68 \cdot 08$ & $6 \cdot 31$ & $17 \cdot 05$ & $8 \cdot 56$ & \\
\hline Picrotoxin & - & 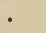 & $\mathrm{C}_{12} \mathrm{H}_{14} \mathrm{O}_{5}$ & $60 \cdot 50$ & $5 \cdot 88$ & $33 \cdot 62$ & & \\
\hline
\end{tabular}




\begin{tabular}{|c|c|c|c|c|c|c|c|c|}
\hline \multicolumn{3}{|c|}{ NaMe. } & \multirow{2}{*}{$\begin{array}{l}\text { ForMula. } \\
\mathrm{C}_{23} \mathrm{H}_{34} \mathrm{~N}_{4} \mathrm{O}_{4}\end{array}$} & \multirow{2}{*}{$\frac{\text { C. }}{64 \cdot 18}$} & \multirow{2}{*}{$\frac{\mathrm{H} .}{7 \cdot 91}$} & \multirow{2}{*}{$\frac{O .}{14 \cdot 89}$} & \multirow{2}{*}{$\frac{N .}{13 \cdot 02}$} & \multirow[t]{2}{*}{ S. } \\
\hline Pilocarpine & & - & & & & & & \\
\hline Pimaric acid & $\cdot$ & - & & $79 \cdot 47$ & $9 \cdot 93$ & $10 \cdot 59$ & & \\
\hline Pinipicrin & • & - & $\mathrm{C}_{22} \mathrm{I}$ & $55 \cdot 46$ & $7 \cdot 56$ & $36 \cdot 98$ & & \\
\hline $\begin{array}{l}\text { Pinite } \\
\text { Piperine }\end{array}$ & & : & $\begin{array}{l}\mathrm{C}_{6}{ }^{3} \\
\mathrm{C}_{17} \mathrm{t}\end{array}$ & $\begin{array}{l}43 \cdot 9 \\
71 \cdot 58\end{array}$ & $\begin{array}{l}7 \cdot 2 \\
6 \cdot 67\end{array}$ & $\begin{array}{l}48 \cdot 9 \\
16 \cdot 84\end{array}$ & & \\
\hline $\begin{array}{l}\text { Piperine } \\
\text { Pipitzahoïc ac }\end{array}$ & & : & ${ }_{0} \mathrm{O}_{3}$ & 72.58 & $\begin{array}{l}6.67 \\
8.06\end{array}$ & $\begin{array}{l}16 \cdot 84 \\
19 \cdot 36\end{array}$ & $4 \cdot 91$ & \\
\hline Populin. & & - & $\mathrm{C}_{20}^{10} \mathrm{~F}$ & $56 \cdot 34$ & $6 \cdot 10$ & $37 \cdot 56$ & & \\
\hline Propionic aci & & • & $\widetilde{C}_{3}$ & $48 \cdot 65$ & $8 \cdot 11$ & $43 \cdot 24$ & & \\
\hline Propyl alcoho & & & & $60 \cdot 00$ & $13 \cdot 33$ & $26 \cdot 66$ & & \\
\hline Protocatechui & $c a c$ & eid & & $54 \cdot 54$ & $3 \cdot 90$ & $41 \cdot 56$ & & \\
\hline Purpurin & & - & $\mathrm{C}_{14}$ & $65 \cdot 62$ & $3 \cdot 13$ & $31 \cdot 25$ & & \\
\hline Pyrocatechin & & - & & $65 \cdot 45$ & $5 \cdot 16$ & $29 \cdot 09$ & & \\
\hline Pyrogallic aci & & - & & $57 \cdot 13$ & $4 \cdot 76$ & $38 \cdot 11$ & & \\
\hline Quassin . & • & • & & $66 \cdot 67$ & $6 \cdot 67$ & $27 \cdot 66$ & & \\
\hline $\begin{array}{l}\text { Quercetin } \\
\text { Quercite }\end{array}$ & - & - & & $59 \cdot 21$ & $3 \cdot 95$ & $\begin{array}{l}36 \cdot 84 \\
48 \cdot 9\end{array}$ & & \\
\hline $\begin{array}{l}\text { Quercite } \\
\text { Quercitrin }\end{array}$ & • & 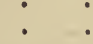 & & $\begin{array}{l}43 \cdot 9 \\
55 \cdot 90\end{array}$ & $\begin{array}{l}7 \cdot 2 \\
4 \cdot 35\end{array}$ & $\begin{array}{l}48 \cdot 9 \\
5 \cdot 75\end{array}$ & & \\
\hline $\begin{array}{l}\text { Quercitrin } \\
\text { Quinamine }\end{array}$ & & & $\mathrm{C}_{19} \stackrel{\mathrm{H}}{\mathrm{H}}$ & $\begin{array}{l}25.90 \\
73 \cdot 08\end{array}$ & $7 \cdot 69$ & 10.25 & $8 \cdot 98$ & \\
\hline Quinine and & Quin & lidine & $\mathrm{C}_{20} \mathrm{H}$ & $75 \cdot 02$ & $6 \cdot 66$ & 10.43 & $8 \cdot 64$ & \\
\hline Racemic acid & & • & & $32 \cdot 0$ & $4 \cdot 0$ & $64 \cdot 0$ & & \\
\hline Rhatania-red & & & & $62 \cdot 17$ & $4 \cdot 81$ & 33.02 & & \\
\hline Rhatania tan & nic a & acid & & $59 \cdot 40$ & $4 \cdot 95$ & $35 \cdot 65$ & & \\
\hline Resorcin & & - & & $65 \cdot 45$ & $5 \cdot 16$ & 29.09 & & \\
\hline Rhinacanthin & & - & $\mathrm{C}_{14} \mathrm{H}$ & $67 \cdot 20$ & $7 \cdot 20$ & $25 \cdot 40$ & & \\
\hline Rhoe & & - & $\mathrm{C}_{21}$ & $\begin{array}{l}65 \cdot 79 \\
79 \cdot 18\end{array}$ & $5 \cdot 48$ & $25 \cdot 08$ & $3 \cdot 65$ & \\
\hline $\begin{array}{l}\text { Ricino } \\
\text { Roccel }\end{array}$ & & - & & $\begin{array}{l}72 \cdot 48 \\
68 \cdot 00\end{array}$ & $11 \cdot 41$ & $\begin{array}{l}16 \cdot 11 \\
21 \cdot 34\end{array}$ & & \\
\hline $\begin{array}{l}\text { Roccellic a } \\
\text { Rottlerin }\end{array}$ & & - & & $\begin{array}{l}68 \cdot 00 \\
71 \cdot 00\end{array}$ & $\begin{array}{l}10 \cdot 66 \\
10 \cdot 05\end{array}$ & $\begin{array}{l}21 \cdot 34 \\
18 \cdot 95\end{array}$ & & \\
\hline Ruberythric a & acid & - & & $54 \cdot 64$ & $5 \cdot 04$ & $40 \cdot 32$ & & \\
\hline Rubia & & - & (?) & $55 \cdot 08$ & $5 \cdot 57$ & $39 \cdot 35$ & & \\
\hline Rubichloric a & cid & - & & $51 \cdot 22$ & $4 \cdot 88$ & $43 \cdot 90$ & & \\
\hline Sabadilline & • & • & $\mathrm{C}_{41} \mathrm{H}$ & $61 \cdot 29$ & $8 \cdot 85$ & $26 \cdot 40$ & $3 \cdot 46$ & \\
\hline Saba & - & . & $\mathrm{C}_{51} \mathrm{H}$ & $61 \cdot 69$ & $8 \cdot 78$ & $26 \cdot 76$ & $2 \cdot 77$ & \\
\hline Salici & & - & $\mathrm{O}_{7}$ & $54: 54$ & $6 \cdot 29$ & $39 \cdot 17$ & & \\
\hline ic acid & & $\cdot$ & & $\begin{array}{l}60.87 \\
68.85\end{array}$ & $\begin{array}{l}4 \cdot 42 \\
4 \cdot 9 ?\end{array}$ & $\begin{array}{l}34 \cdot 78 \\
26 \cdot 23\end{array}$ & & \\
\hline $\begin{array}{l}\text { ous aci } \\
\text { inarine }\end{array}$ & d. & $\dot{.}$ & $\mathrm{C}_{70}$ & $\begin{array}{l}68 \cdot 85 \\
70 \cdot 59\end{array}$ & $\begin{array}{l}4 \cdot 92 \\
5 \cdot 26\end{array}$ & $\left|\begin{array}{l}26 \cdot 23 \\
19 \cdot 82\end{array}\right|$ & $4 \cdot 33$ & \\
\hline $\begin{array}{l}\text { Sanguinarine } \\
\text { Santalin }\end{array}$ & - & . & (?) & $65 \cdot 69$ & $5 \cdot 11$ & $\begin{array}{l}1902 \\
29 \cdot 20\end{array}$ & 400 & \\
\hline Santonin & - & 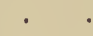 & & $73 \cdot 17$ & $7 \cdot 32$ & $19 \cdot 51$ & & \\
\hline Sapc & - & • & $\mathrm{C}_{52} \mathrm{H}_{86} \mathrm{O}_{26}(?)$ & $55 \cdot 4$ & $7 \cdot 6$ & 36.9 & & \\
\hline Scleromucin & - & 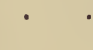 & & $29 \cdot 67$ & $6 \cdot 44$ & ? & $6 \cdot 41$ & $\begin{array}{l}(26 \cdot 8 \\
\text { ash) }\end{array}$ \\
\hline Scleroxanthin & & - & $\mathrm{C}_{10} \mathrm{I}$ & $61 \cdot 8$ & $5 \cdot 1$ & $32 \cdot 0$ & & \\
\hline acid & & - & & & $5 \cdot 2$ & $50 \cdot 6$ & $4 \cdot 2$ & \\
\hline in & & - & (?) & $58 \cdot 06$ & $5 \cdot 06$ & $36 \cdot 87$ & & \\
\hline Sinalbin & e 0 & $\dot{\sin }$ & $\mathrm{C}_{30} \mathrm{H}$ & $47 \cdot 87$ & $5 \cdot 85$ & $34 \cdot 05$ & $3 \cdot 72$ & $\begin{array}{l}8.51 \\
8.69\end{array}$ \\
\hline $\begin{array}{l}\text { Sulphocyanid } \\
\text { Sinistrin }\end{array}$ & e 0 & sina & $\mathrm{C}_{17} \mathrm{H}$ & $\begin{array}{l}55 \cdot 43 \\
44 \cdot 44\end{array}$ & 6.53 & $\begin{array}{l}21 \cdot 74 \\
49 \cdot 39\end{array}$ & $7 \cdot 61$ & \\
\hline $\begin{array}{l}\text { Sinistrin } \\
\text { Socaloïn }\end{array}$ & . & . & $\begin{array}{l}\mathbf{C}_{6} \\
\mathrm{C}_{1}\end{array}$ & $\begin{array}{l}44 \cdot 44 \\
59 \cdot 63\end{array}$ & $\begin{array}{l}6 \cdot 17 \\
5 \cdot 59\end{array}$ & $\begin{array}{l}49 \cdot 39 \\
34 \cdot 78\end{array}$ & & \\
\hline ine & - & - & $\mathrm{C}_{42} \mathrm{H}_{87} \mathrm{NO}_{15}$ & $60 \cdot 66$ & $8 \cdot 78$ & $28 \cdot 88$ & $1 \cdot 68$ & \\
\hline & - & - & & $40 \cdot 00$ & $6 \cdot 66$ & $53 \cdot 34$ & & \\
\hline Sparteïne & - & • & $\mathrm{C}_{8} \mathrm{H}_{13} \mathrm{~N}$ & $78 \cdot 05$ & 10.57 & & $11 \cdot 38$ & \\
\hline Staphisagrine & & • & $\mathrm{C}_{20} \mathrm{H}_{32} \mathrm{NO}_{5}$ & $67 \cdot 5$ & $8 \cdot 4$ & $20 \cdot 5$ & $3 \cdot 6$ & \\
\hline Starc & 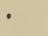 & & $\mathrm{C}_{6} \mathrm{H}_{10} \mathrm{O}_{5}$ & $44 \cdot 44$ & $6 \cdot 17$ & $49 \cdot 39$ & & \\
\hline Stearic acid & - & - & $\mathrm{C}_{18} \mathrm{H}_{36} \mathrm{O}_{2}$ & 76.06 & $12 \cdot 68$ & $11 \cdot 26$ & & \\
\hline Strychnine & - & 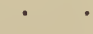 & $\mathrm{C}_{21} \mathrm{H}_{22} \mathrm{~N}_{2} \mathrm{O}_{2}$ & $77 \cdot 24$ & $6 \cdot 54$ & $7 \cdot 30$ & $8 \cdot 92$ & \\
\hline $\begin{array}{l}\text { Styracin } \\
\text { Styrol . }\end{array}$ & & : & $\begin{array}{c}\mathrm{C}_{13} \mathrm{H}_{16} \mathrm{O}_{2} \\
\mathrm{C}_{8} \mathrm{H}_{8}\end{array}$ & $\begin{array}{l}81 \cdot 82 \\
92 \cdot 31\end{array}$ & $\begin{array}{l}6 \cdot 06 \\
7 \cdot 69\end{array}$ & $12 \cdot 12$ & & \\
\hline yrol • & & & & & 100 & & & \\
\hline
\end{tabular}




\begin{tabular}{|c|c|c|c|c|c|c|}
\hline Name. & Formula. & C. & H. & O. & N. & S. \\
\hline 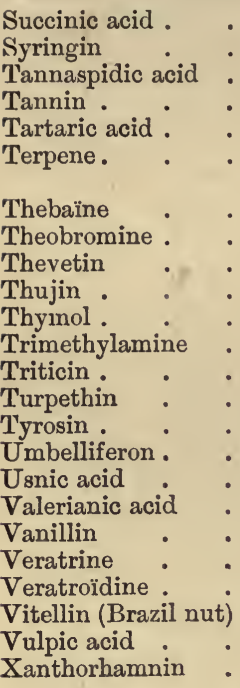 & $\begin{array}{c}\mathrm{C}_{4} \mathrm{H}_{6} \mathrm{O}_{4} \\
\mathrm{C}_{19} \mathrm{H}_{28} \mathrm{O}_{5} \\
\mathrm{C}_{26} \mathrm{H}_{28} \mathrm{O}_{11} \\
\mathrm{C}_{27} \mathrm{H}_{22} \mathrm{O}_{17} \\
\mathrm{C}_{4} \mathrm{H}_{6} \mathrm{O}_{6} \\
\mathrm{C}_{10} \mathrm{H}_{16}, \mathrm{C}_{15} \mathrm{H}_{24}, \\
\text { and } \mathrm{C}_{20} \mathrm{H}_{32} \\
\mathrm{C}_{19} \mathrm{H}_{21} \mathrm{NO}_{3} \\
\mathrm{C}_{7} \mathrm{H}_{8} \mathrm{~N}_{4} \mathrm{O}_{2} \\
\mathrm{C}_{54} \mathrm{H}_{84} \mathrm{O}_{24} \\
\mathrm{C}_{20} \mathrm{H}_{22} \mathrm{O}_{12} \\
\mathrm{C}_{10} \mathrm{H}_{14} \mathrm{O} \\
\mathrm{C}_{3} \mathrm{H}_{9} \mathrm{~N} \\
\mathrm{C}_{12} \mathrm{H}_{22} \mathrm{O}_{11} \\
\mathrm{C}_{34} \mathrm{H}_{56} \mathrm{O}_{16} \\
\mathrm{C}_{9} \mathrm{H}_{11} \mathrm{NO}_{8} \\
\mathrm{C}_{9} \mathrm{H}_{6} \mathrm{O}_{3} \\
\mathrm{C}_{18} \mathrm{H}_{18} \mathrm{O}_{8} \\
\mathrm{C}_{5} \mathrm{H}_{10} \mathrm{O}_{2} \\
\mathrm{C}_{8} \mathrm{H}_{8} \mathrm{O}_{3} \\
\mathrm{C}_{52} \mathrm{H}_{86} \mathrm{~N}_{2} \mathrm{O}_{15}(?) \\
\mathrm{C}_{24} \mathrm{H}_{37} \mathrm{NO}_{7}(?) \\
? \\
\mathrm{C}_{17} \mathrm{H}_{14} \mathrm{O}_{5} \\
\mathrm{C}_{48} \mathrm{H}_{66} \mathrm{O}_{29}\end{array}$ & $\begin{array}{l}40 \cdot 68 \\
54 \cdot 81 \\
60 \cdot 46 \\
52 \cdot 42 \\
32 \cdot 0 \\
88 \cdot 23 \\
73 \cdot 31 \\
46 \cdot 67 \\
58 \cdot 06 \\
52 \cdot 86 \\
80 \cdot 00 \\
61 \cdot 02 \\
42 \cdot 10 \\
56 \cdot 66 \\
59 \cdot 66 \\
66 \cdot 66 \\
59 \cdot 39 \\
58 \cdot 82 \\
63 \cdot 13 \\
64 \cdot 42 \\
63 \cdot 8 \\
52 \cdot 29 \\
70 \cdot 81 \\
51 \cdot 08\end{array}$ & $\begin{array}{c}5 \cdot 09 \\
6 \cdot 73 \\
5 \cdot 42 \\
3 \cdot 56 \\
4 \cdot 0 \\
\\
11 \cdot 77 \\
6 \cdot 75 \\
4 \cdot 44 \\
7 \cdot 53 \\
4 \cdot 84 \\
9 \cdot 33 \\
15 \cdot 25 \\
6 \cdot 43 \\
7 \cdot 77 \\
6 \cdot 07 \\
3 \cdot 71 \\
4 \cdot 94 \\
9 \cdot 80 \\
5 \cdot 26 \\
8 \cdot 70 \\
8 \cdot 2 \\
7 \cdot 24 \\
4 \cdot 35 \\
5 \cdot 83\end{array}$ & $\begin{array}{l}15 \cdot 44 \\
17 \cdot 78 \\
34 \cdot 41 \\
42 \cdot 30 \\
10 \cdot 77 \\
\ldots 7 \\
51 \cdot 47 \\
35 \cdot 57 \\
26 \cdot 54 \\
29 \cdot 63 \\
35 \cdot 36 \\
31 \cdot 37 \\
31 \cdot 58 \\
23 \cdot 97 \\
24 \cdot 9 \\
21 \cdot 06 \\
24 \cdot 84 \\
43 \cdot 09\end{array}$ & $\begin{array}{c}2 \cdot 91 \\
3 \cdot 1 \\
18 \cdot 09\end{array}$ & $1 \cdot 32$ \\
\hline
\end{tabular}


COMPOSITION OF THE MORE IMPORTANT CONSTITUENTS OF PLANTS, ARRANGED ACCORDING TO PERCENTAGE OF CARBON.

\begin{tabular}{|c|c|c|c|c|c|}
\hline C. & $\mathrm{H}$. & O. & N. & S. & Name. \\
\hline $26 \cdot 66$ & $2 \cdot 22$ & $71 \cdot 11$ & & & Oxalic acid. \\
\hline $31 \cdot 58$ & $5 \cdot 26$ & $63 \cdot 16$ & & & Glycolic acid. \\
\hline $31 \cdot 81$ & $5 \cdot 04$ & $42 \cdot 42$ & $3 \cdot 72$ & $16 \cdot 99$ & Myronic acid. \\
\hline $32 \cdot 00$ & $4 \cdot 00$ & $64 \cdot 00$ & $\ldots$ & ... & Tartaric and racemic acid. \\
\hline $35 \cdot 82$ & $4 \cdot 48$ & $59 \cdot 70$ & 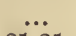 & ... & Malic acid. \\
\hline $36 \cdot 36$ & $6 \cdot 06$ & $36 \cdot 37$ & $21 \cdot 21$ & ... & Asparagine. \\
\hline $37 \cdot 50$ & $4 \cdot 17$ & $58 \cdot 33$ & $\ldots$ & ... & Citric acid. \\
\hline $37 \cdot 50$ & $12 \cdot 50$ & $50 \cdot 00$ & 극 & $\cdots$ & Methylic alcohol. \\
\hline $38 \cdot 71$ & $16 \cdot 13$ & $\ldots \ddot{77}$ & $45 \cdot 17$ & $\cdots$ & Methylamine. \\
\hline $\begin{array}{l}39 \cdot 13 \\
39 \cdot 34\end{array}$ & $8 \cdot 70$ & $\begin{array}{l}52 \cdot 17 \\
52 \cdot 46\end{array}$ & $\cdots$ & $\cdots$ & \\
\hline 39.56 & $7 \cdot 69$ & 52.75 & $\cdots$ & $\cdots$ & Dulcite, isodulcite, mannite, \\
\hline 40.00 & $6 \cdot 66$ & 53.33 & ... & ... & $\begin{array}{l}\text { Acetic and lactic acid, glu- } \\
\text { cose, etc. }\end{array}$ \\
\hline 40.00 & $5 \cdot 2$ & $50 \cdot 6$ & $4 \cdot 2$ & $\cdots$ & Sclerotic acid. \\
\hline $40 \cdot 68$ & $5 \cdot 09$ & $54 \cdot 23$ & $\ldots$ & ... & Succinic acid. \\
\hline $41 \cdot 38$ & $3 \cdot 45$ & $55 \cdot 17$ & ... & ... & Aconitic acid. \\
\hline $41 \cdot 38$ & 3.45 & $55 \cdot 17$ & ... & ... & Fumaric and maleïc acid. \\
\hline $42 \cdot 0$ & $2 \cdot 0$ & $56 \cdot 0$ & ... & ... & Meconic acid. \\
\hline $42 \cdot 10$ & $6 \cdot 43$ & $51 \cdot 47$ & ... & $\ldots$ & $\begin{array}{l}\text { Arabic and metarabic acid, } \\
\text { pararabin, triticin, saccha } \\
\text { rose, etc. }\end{array}$ \\
\hline $43 \cdot 75$ & $6 \cdot 30$ & $50 \cdot 19$ & .. & ... & Kinic acid. \\
\hline 43.9 & $7 \cdot 2$ & $48 \cdot 9$ & $\ldots$ & $\ldots$ & Pinite and quercite. \\
\hline $44 \cdot 21$ & $5 \cdot 26$ & 50.53 & & ... & Boheic acid, \\
\hline $44 \cdot 44$ & $9 \cdot 63$ & $35 \cdot 55$ & $10 \cdot 37$ & ... & Betaïne. \\
\hline $44 \cdot 44$ & $3 \cdot 70$ & & $51 \cdot 85$ & ... & Hydrocyanic acid. \\
\hline $44 \cdot 44$ & $4 \cdot 76$ & $51 \cdot 80$ & ... & $\cdots$ & Cyclopin. \\
\hline $44 \cdot 44$ & $6 \cdot 17$ & $49 \cdot 39$ & $\ldots$ & & $\begin{array}{l}\text { Cellulose, dextrin, inulin, } \\
\text { levulin, sinistrin, starch. }\end{array}$ \\
\hline $44 \cdot 84$ & $5 \cdot 33$ & $49 \cdot 83$ & ... & ... & Cinchona-tannic acid. \\
\hline $45 \cdot 65$ & $2 \cdot 17$ & $52 \cdot 24$ & & ... & Chelidonic acid. \\
\hline $46 \cdot 67$ & $4 \cdot 44$ & $17 \cdot 78$ & $31 \cdot 11$ & & Theobromine. \\
\hline $47 \cdot 87$ & $5 \cdot 85$ & $34 \cdot 05$ & $3 \cdot 72$ & $8 \cdot 51$ & Sinalbine. \\
\hline $48 \cdot 65$ & $8 \cdot 11$ & $43 \cdot 24$ & & ... & Propionic acid. \\
\hline $49 \cdot 48$ & $5 \cdot 15$ & 16.51. & $28 \cdot 86$ & $\ldots$ & Caffeine. \\
\hline $49 \cdot 59$ & $12 \cdot 39$ & $26 \cdot 44$ & $11 \cdot 57$ & $\ldots$ & Choline. \\
\hline $49 \cdot 60$ & $4 \cdot 92$ & $43 \cdot 26$ & $2 \cdot 22$ & & Indican. \\
\hline $50 \cdot 24$ & $6 \cdot 81$ & $24 \cdot 13$ & $18 \cdot 37$ & 0.45 & Conglutin. \\
\hline $50 \cdot 42$ & $10 \cdot 92$ & $26 \cdot 89$ & $11 \cdot 77$ & & Muscarine. \\
\hline $51 \cdot 0$ & 6.7 & $25 \cdot 4$ & $16 \cdot 1$ & 0.8 & Glutencaseïn. \\
\hline $51 \cdot 00$ & $7 \cdot 00$ & $42 \cdot 00$ & .. & ... & Ericolin. \\
\hline $51 \cdot 02$ & $4 \cdot 89$ & $44 \cdot 87$ & ... & $\ldots$ & Fraxin. \\
\hline $51 \cdot 22$ & $4 \cdot 88$ & 43.90 & & $\ldots$ & Rubichloric acid. \\
\hline $51 \cdot 47$ & $7 \cdot 02$ & $24 \cdot 29$ & $16 \cdot 82$ & 0.40 & Legumin. \\
\hline $52 \cdot 01$ & $5 \cdot 88$ & $42 \cdot 11$ & & $\because \ddot{0}$ & Cinchona-tannic acid. \\
\hline $52 \cdot 07$ & $7 \cdot 24$ & $21 \cdot 06$ & 18.09 & $1 \cdot 32$ & Vitellin. \\
\hline
\end{tabular}




\begin{tabular}{|c|c|c|c|c|c|}
\hline C. & H. & O. & $\mathrm{N}$ & S. & NaMe. \\
\hline $\begin{array}{l}52 \cdot 39 \\
52 \cdot 42\end{array}$ & $\begin{array}{l}4 \cdot 78 \\
3 \cdot 56\end{array}$ & $42 \cdot 83$ & $\cdots$ & $\cdots$ & Daphnin. \\
\hline $\begin{array}{l}52 \cdot 42 \\
52 \cdot 45\end{array}$ & $\begin{array}{l}3 \cdot 56 \\
6 \cdot 81\end{array}$ & $\begin{array}{l}44 \cdot 02 \\
22 \cdot 21\end{array}$ & $10 \ddot{6} \cdot 65$ & $\ddot{0.8}$ & $\begin{array}{l}\text { Gallotannic acid. } \\
\text { Albumin. }\end{array}$ \\
\hline $52 \cdot 47$ & $5 \cdot 79$ & $40 \cdot 23$ & 1.53 & $\ldots$ & $\begin{array}{l}\text { Albumin. } \\
\text { Laurocerasin. }\end{array}$ \\
\hline $52 \cdot 51$ & $5 \cdot 91$ & $38 \cdot 52$ & 3.06 & $\ldots$ & Amygdalin. \\
\hline $52 \cdot 53$ & $7 \cdot 38$ & $40 \cdot 27$ & & & Helleborein. \\
\hline $52 \cdot 60$ & $7 \cdot 00$ & $21 \cdot 49$ & 1806 & 0.85 & Gliadin. \\
\hline $52 \cdot 86$ & $4 \cdot 84$ & $42 \cdot 30$ & $\ldots$ & $\ldots$ & Thujin. \\
\hline 52.9 & $5 \cdot 2$ & $41 \cdot 9$ & ... & ... & Apiin. \\
\hline $53 \cdot 21$ & $7 \cdot 60$ & $39 \cdot 19$ & ... & ... & Digitonin. \\
\hline $53 \cdot 33$ & $5 \cdot 19$ & $41 \cdot 48$ & $\ldots$ & $\cdots$ & Cinchona-red. \\
\hline $53 \cdot 7$ & $6 \cdot 1$ & $40 \cdot 2$ & $\cdots$ & $\cdots$ & Arbutin. \\
\hline $53 \cdot 85$ & $5 \cdot 13$ & $41 \cdot 02$ & $\cdots$ & $\cdots$ & \\
\hline $\begin{array}{l}53 \cdot 91 \\
54.08\end{array}$ & $\begin{array}{l}8 \cdot 59 \\
4 \cdot 72\end{array}$ & $\begin{array}{l}37 \cdot 50 \\
41 \cdot 20\end{array}$ & $\begin{array}{l}\cdots \\
\ldots\end{array}$ & $\begin{array}{l}\cdots \\
\ldots\end{array}$ & $\begin{array}{l}\text { Convallamarin. } \\
\text { Datiscin. }\end{array}$ \\
\hline $54 \cdot 11$ & 6.90 & $21 \cdot 48$ & $16 \cdot 63$ & 0.88 & Mucedin. \\
\hline $54 \cdot 54$ & 3.90 & $41 \cdot 56$ & ... & $\ldots$ & Protocatechuic acid. \\
\hline 54.55 & $9 \cdot 09$ & $36 \cdot 36$ & ... & $\ldots$ & Butyric acid. \\
\hline $54 \cdot 54$ & $6 \cdot 29$ & $39 \cdot 17$ & $\ldots$ & ... & Salicin. \\
\hline $54 \cdot 63$ & $5 \cdot 47$ & $39 \cdot 90$ & ... & ... & Lupinin. \\
\hline $54 \cdot 64$ & $5 \cdot 04$ & $40 \cdot 32$ & $\ldots$ & $\ldots$ & Ruberythric acid. \\
\hline $54 \cdot 77$ & $5 \cdot 39$ & $39 \cdot 84$ & ... & $\cdots$ & Hesperidin. \\
\hline $54 \cdot 81$ & $6 \cdot 73$ & $38 \cdot 46$ & ... & $\ldots$ & Syringin. \\
\hline $54 \cdot 87$ & $7 \cdot 37$ & $37 \cdot 50$ & $\ldots$ & $\ldots$ & Convolvulin. \\
\hline $54 \cdot 96$ & 9.92 & $24 \cdot 43$ & $10 \cdot 69$ & ... & Leucine. \\
\hline $55 \cdot 08$ & $5 \cdot 57$ & $39 \cdot 35$ & ... & ... & Rubian. \\
\hline $55 \cdot 29$ & $7 \cdot 83$ & $36 \cdot 87$ & $\ldots$ & $\ldots$ & Cyclamin. \\
\hline $55 \cdot 4$ & $7 \cdot 6$ & $36 \cdot 9$ & $\because$ & ... & Saponin. \\
\hline $55 \cdot 43$ & $6 \cdot 53$ & $21 \cdot 74$ & $7 \cdot 61$ & $8 \cdot 69$ & Sulphocyanate of sinapine. \\
\hline $55 \cdot 46$ & $7 \cdot 56$ & $36 \cdot 98$ & $\cdots$ & $\cdots$ & Menyanthin. \\
\hline $55 \cdot 46$ & $7 \cdot 56$ & $36 \cdot 98$ & $\cdots$ & ... & Pinipicrin. \\
\hline $55 \cdot 6$ & $5 \cdot 6$ & $38 \cdot 8$ & $\cdots$ & $\cdots$ & Naringin. \\
\hline $55 \cdot 81$ & 6.99 & $37 \cdot 20$ & $\cdots$ & $\cdots$ & Crotonic acid. \\
\hline $55 \cdot 63$ & 1.99 & $42 \cdot 38$ & $\cdots$ & $\cdots$ & Ellagic acid. \\
\hline $55 \cdot 90$ & $4 \cdot 35$ & $39 \cdot 75$ & ... & ... & Quercitrin. \\
\hline $56 \cdot 14$ & $6 \cdot 43$ & $37 \cdot 43$ & $\cdots$ & .. & Coniferin. \\
\hline $56 \cdot 15$ & $5 \cdot 81$ & $38 \cdot 05$ & $\cdots$ & $\cdots$ & Phlorizin. \\
\hline $56 \cdot 25$ & $3 \cdot 75$ & 40.00 & ... & $\ldots$ & Maclurin. \\
\hline $56 \cdot 34$ & $6 \cdot 10$ & $37 \cdot 56$ & ... & $\cdots$ & Populin. \\
\hline $56 \cdot 37$ & 6.04 & $37 \cdot 59$ & ... & $\ldots$ & Ipecacuanha-tannic acid. \\
\hline 56.66 & $7 \cdot 77$ & $35 \cdot 57$ & ... & $\cdots$ & Jalapin and turpethin. \\
\hline $56 \cdot 75$ & $5 \cdot 41$ & $37 \cdot 84$ & ... & ... & Caffeo-tannic acid. \\
\hline $56 \cdot 75$ & $5 \cdot 40$ & $37 \cdot 85$ & ... & ... & Carthamin. \\
\hline $57 \cdot 13$ & $4 \cdot 76$ & $38 \cdot 11$ & ... & ... & Phloroglucin, pyrogallol, etc. \\
\hline $57 \cdot 15$ & $4 \cdot 76$ & $38 \cdot 09$ & ... & .. & Orsellic acid. \\
\hline $57 \cdot 32$ & $7 \cdot 01$ & $35 \cdot 67$ & & & Globularin. \\
\hline $57 \cdot 57$ & $5 \cdot 12$ & $34 \cdot 96$ & $1 \cdot 50$ & 0.85 & Cathartic acid. \\
\hline $57 \cdot 64$ & $7 \cdot 42$ & 34.94 & & ... & I)ulcamarin. \\
\hline $57 \cdot 69$ & $13 \cdot 46$ & $15 \cdot 38$ & $13 \cdot 46$ & ... & Amanitine. \\
\hline $58 \cdot 00$ & $5 \cdot 06$ & $36 \cdot 87$ & $\ldots$ & $\ldots$ & Scoparin. \\
\hline $58 \cdot 06$ & $7 \cdot 53$ & $34 \cdot 41$ & $\ldots$ & $\ldots$ & Thevetin. \\
\hline $58 \cdot 22$ & $5 \cdot 50$ & $36 \cdot 28$ & ... & ... & Curaçao-aloin. \\
\hline $58 \cdot 23$ & $4 \cdot 64$ & $37 \cdot 13$ & $\ldots$ & $\ldots$ & Chrysorhamnin. \\
\hline $58 \cdot 24$ & $7 \cdot 38$ & $34 \cdot 38$ & $\ldots$ & $\ldots$ & Caïncin. \\
\hline $58 \cdot 82$ & $9 \cdot 80$ & $31 \cdot 37$ & $\ldots$ & ... & Valerianic acid. \\
\hline $59 \cdot 21$ & $3 \cdot 91$ & $36 \cdot 84$ & $\cdots$ & $\cdots$ & Quercetin, \\
\hline $59 \cdot 26$ & $6 \cdot 17$ & 34.57 & & ... & Capaloin. \\
\hline
\end{tabular}




\begin{tabular}{|c|c|c|c|c|c|c|}
\hline C. & $\mathrm{H}$. & O. & N. & S. & NaMe. & \\
\hline $59 \cdot 34$ & $5 \cdot 49$ & $35 \cdot 17$ & $\cdots$ & $\cdots$ & Everninic acid. & K \\
\hline $59 \cdot 44$ & $5 \cdot 88$ & $34 \cdot 68$ & $\ldots$ & $\ldots$ & Nataloïn. & \\
\hline $59 \cdot 40$ & $4 \cdot 95$ & $35 \cdot 65$ & $\ldots$ & $\ldots$ & Rhatania-tannic acid & \\
\hline $59 \cdot 39$ & $4 \cdot 94$ & $35 \cdot 36$ & $\ldots$ & $\cdots$ & Usnic acid. & \\
\hline $59 \cdot 63$ & $5 \cdot 59$ & $34 \cdot 78$ & … & $\cdots$ & Socaloün. & \\
\hline $59 \cdot 63$ & $6 \cdot 28$ & $31 \cdot 09$ & $3 \cdot 02$ & $\cdots$ & Narceine. & \\
\hline $\begin{array}{l}59 \cdot 66 \\
59 \cdot 78\end{array}$ & $6 \cdot 07$ & 26.54 & $7 \cdot 73$ & $\cdots$ & Tyrosine. & \\
\hline $\begin{array}{l}59 \cdot 78 \\
59 \cdot 80\end{array}$ & $\begin{array}{l}7 \cdot 47 \\
5 \cdot 64\end{array}$ & $\begin{array}{l}32 \cdot 75 \\
34 \cdot 56\end{array}$ & $\begin{array}{l}\ldots \\
\ldots\end{array}$ & $\begin{array}{l}\cdots \\
\ldots\end{array}$ & $\begin{array}{l}\text { Colocynthin. } \\
\text { Ononin. }\end{array}$ & \\
\hline $59 \cdot 92$ & $7 \cdot 05$ & $32 \cdot 66$ & $\dddot{1} \cdot 17$ & $\cdots$ & Glycyrrhizic acid. & \\
\hline $59 \cdot 95$ & 8.05 & 32.00 & $\ldots$ & $\ldots$ & Digitalin. & \\
\hline $60 \cdot 00$ & $8 \cdot 00$ & $32 \cdot 00$ & $\ldots$ & $\ldots$ & Angelic acid. & . \\
\hline $60 \cdot 00$ & $8 \cdot 33$ & $31 \cdot 67$ & $\ldots$ & $\ldots$ & Bryonin. & \\
\hline $60 \cdot 00$ & $4 \cdot 44$ & $35 \cdot 56$ & $\ldots$ & $\cdots$ & Cetraric acid. & \\
\hline 60.00 & $13 \cdot 33$ & $26 \cdot 67$ & $\ldots$ & $\cdots$ & Propylic alcohol. & \\
\hline $60 \cdot 37$ & $4 \cdot 40$ & $35 \cdot 23$ & $\ldots$ & $\ldots$ & Lecanoric acid. & \\
\hline $60 \cdot 40$ & $9 \cdot 0$ & $30 \cdot 6$ & $\ldots$ & $\ldots$ & Parillin. & \\
\hline $60 \cdot 46$ & $5 \cdot 42$ & $34 \cdot 12$ & $\ldots$ & $\cdots$ & Tannaspidic acid. & \\
\hline $60 \cdot 50$ & $5 \cdot 88$ & $33 \cdot 62$ & \#ir & $\cdots$ & Picrotoxin. & \\
\hline 60.53 & $6 \cdot 82$ & $28 \cdot 50$ & $4 \cdot 15$ & $\cdots$ & Colchicine. & \\
\hline 60.57 & $7 \cdot 26$ & $27 \cdot 76$ & $4 \cdot 42$ & $\ldots$ & Oxyacanthine. & \\
\hline $60 \cdot 66$ & $8 \cdot 78$ & $28 \cdot 88$ & $1 \cdot 68$ & $\cdots$ & Solanine. & \\
\hline $60 \cdot 67$ & $3 \cdot 37$ & $35 \cdot 96$ & $\cdots$ & $\cdots$ & Parellic acid. & \\
\hline 60.67 & $6 \cdot 37$ & $32 \cdot 96$ & ... & $\ldots$ & Philyrin. & \\
\hline $60 \cdot 71$ & $5 \cdot 95$ & $33 \cdot 34$ & ... & $\ldots$ & Barbaloïn. & \\
\hline $60 \cdot 81$ & $4 \cdot 90$ & $34 \cdot 29$ & ... & $\cdots$ & Gyrophoric acid. & \\
\hline 60.85 & $4 \cdot 75$ & $34 \cdot 40$ & $\cdots$ & $\cdots$ & Gardenin. & \\
\hline $60 \cdot 86$ & $4 \cdot 42$ & 34.78 & ... & $\ldots$ & Salicylic acid. & \\
\hline $60 \cdot 90$ & $4 \cdot 81$ & $34 \cdot 23$ & ‥ & $\ldots$ & Catechin. & \\
\hline $61 \cdot 02$ & $15 \cdot 25$ & & 23.73 & $\cdots$ & Trimethylamine. & \\
\hline $61 \cdot 03$ & $8 \cdot 56$ & $25 \cdot 27$ & $5 \cdot 14$ & $\cdots$ & Jervine. & \\
\hline $61 \cdot 29$ & $8 \cdot 85$ & $26 \cdot 40$ & $3 \cdot 46$ & $\ldots$ & Sabadilline. & \\
\hline $61 \cdot 39$ & $6 \cdot 67$ & $29 \cdot 77$ & $2 \cdot 17$ & $\cdots$ & Aconitine. & \\
\hline $61 \cdot 44$ & $4 \cdot 82$ & $33 \cdot 74$ & $\ddot{0}$ & $\cdots$ & Evernic acid. & \\
\hline $61 \cdot 69$ & $8 \cdot 78$ & $26 \cdot 76$ & $2 \cdot 77$ & $\ldots$ & Sabatrine. & \\
\hline $61 \cdot 8$ & $5 \cdot 1$ & $32 \cdot 0$ & $\ldots$ & $\cdots$ & Scleroxanthin. & \\
\hline $61 \cdot 85$ & $5 \cdot 15$ & 33.00 & $\cdots$ & $\cdots$ & Meconin. & \\
\hline $61 \cdot 86$ & $5 \cdot 15$ & $32 \cdot 99$ & $\cdots$ & $\cdots$ & Benzohelicin. & \\
\hline $6 \cdot 2 \cdot 01$ & $5 \cdot 15$ & 33.64 & $\ldots$ & $\ldots$ & Cinchona-nova-red. & \\
\hline $62 \cdot 07$ & $10 \cdot 35$ & $27 \cdot 58$ & $\ldots$ & $\cdots$ & Caproic acid. & \\
\hline $62 \cdot 07$ & $3 \cdot 45$ & $34 \cdot 48$ & $\ldots$ & .. & Luteolin. & \\
\hline $62 \cdot 17$ & $8 \cdot 81$ & $29 \cdot 02$ & $\ldots$ & $\ldots$ & Gratiolin. & \\
\hline $62 \cdot 17$ & $4 \cdot 81$ & 33.02 & $\ldots$ & $\ldots$ & Rhatania-red. & \\
\hline $62 \cdot 33$ & $6 \cdot 49$ & $31 \cdot 17$ & $\ldots$ & $\ldots$ & Crocin. & \\
\hline $62 \cdot 4$ & $9 \cdot 0$ & $28 \cdot 6$ & $\ldots$ & $\ldots$ & Melanthin. & \\
\hline $62 \cdot 46$ & $4 \cdot 66$ & $32 \cdot 88$ & $\ldots$ & $\ldots$ & Catechu-tannic acid. & \\
\hline $62 \cdot 50$ & $4 \cdot 17$ & $33 \cdot 33$ & ... & $\cdots$ & Anemonin. & \\
\hline $62 \cdot 68$ & $7 \cdot 46$ & $29 \cdot 88$ & ... & $\ldots$ & Antiarin. & \\
\hline $62 \cdot 92$ & $4 \cdot 72$ & $32 \cdot 36$ & $\ldots$ & $\ldots$ & Linin. & \\
\hline $63 \cdot 00$ & $7 \cdot 0$ & $30 \cdot 0$ & $\ldots$ & $\ldots$ & Cnicin. & \\
\hline 63.09 & $7 \cdot 47$ & $27 \cdot 32$ & $2 \cdot 12$ & $\ldots$ & Nepaline. & \\
\hline $63 \cdot 13$ & $5 \cdot 26$ & $30 \cdot 58$ & $\ldots$ & $\ldots$ & Vanillin. & \\
\hline $63 \cdot 16$ & $9 \cdot 60$ & $27 \cdot 24$ & $\ldots$ & $\ldots$ & Convallarin. & \\
\hline $63 \cdot 44$ & 6.58 & $25 \cdot 20$ & $4 \cdot 38$ & $\ldots$ & Colchiceïne. & \\
\hline $63 \cdot 57$ & $4 \cdot 63$ & $31 \cdot 79$ & $\ldots$ & $\ldots$ & Hæmatoxylin. & \\
\hline $63 \cdot 60$ & $8 \cdot 50$ & $27 \cdot 90$ & ... & $\ldots$ & Uigitoxin. & \\
\hline $63 \cdot 64$ & $6 \cdot 06$ & $30 \cdot 30$ & $\ldots$ & $\ldots$ & Physalin. & \\
\hline
\end{tabular}




\begin{tabular}{|c|c|c|c|c|c|}
\hline C. & H. & 0. & N. & S. & Name. \\
\hline $63 \cdot 83$ & $6 \cdot 38$ & $29 \cdot 76$ & & & Coriamyrtin. \\
\hline $63 \cdot 8$ & $8 \cdot 2$ & $24 \cdot 9$ & $3 \cdot 1$ & $\begin{array}{l}\cdots \\
\cdots\end{array}$ & Veratroidine. \\
\hline 63.92 & $5 \cdot 57$ & $27 \cdot 12$ & $3 \cdot 39$ & $\cdots$ & Narcotine. \\
\hline $64 \cdot 12$ & $11 \cdot 44$ & $24 \cdot 44$ & $\ldots$ & $\ldots$ & Oenanthic acid. \\
\hline $64 \cdot 20$ & $6 \cdot 17$ & 29.63 & $\cdots$ & $\begin{array}{l}\cdots \\
\cdots\end{array}$ & Filicin. \\
\hline $64 \cdot 23$ & $8 \cdot 77$ & $27 \cdot 00$ & & $\begin{array}{l}\cdots \\
\cdots\end{array}$ & Ceric acid. \\
\hline $64 \cdot 42$ & $8 \cdot 70$ & $23 \cdot 97$ & $2 \cdot 91$ & $\ldots$ & Veratrine. \\
\hline $64 \cdot 48$ & $4 \cdot 40$ & $31 \cdot 20$ & & $\ldots$ & Kämpferid. \\
\hline $64 \cdot 55$ & $8 \cdot 66$ & $23 \cdot 47$ & $3 \cdot 42$ & $\begin{array}{l}\cdots \\
\cdots\end{array}$ & Delphinine. \\
\hline $64 \cdot 80$ & $13 \cdot 51$ & $21 \cdot 62$ & $\ldots$ & $\ldots$ & Butylic alcohol. \\
\hline $64 \cdot 90$ & $7 \cdot 6$ & $27 \cdot 5$ & ... & $\ldots$ & Cäilcedrin. \\
\hline $65 \cdot 11$ & $3 \cdot 87$ & $31 \cdot 02$ & ... & $\ldots$ & Gentisin. \\
\hline $65 \cdot 26$ & $6 \cdot 66$ & $28 \cdot 07$ & ... & $\ldots$ & Kosin. \\
\hline $65 \cdot 28$ & $5 \cdot 60$ & $29 \cdot 03$ & ... & $\cdots$ & Columbin. \\
\hline $65 \cdot 45$ & $5 \cdot 16$ & $29 \cdot 09$ & ... & $\cdots$ & $\begin{array}{l}\text { Pyrocatechin, hydroqui- } \\
\text { none, resorcin, etc. }\end{array}$ \\
\hline $65 \cdot 49$ & $7 \cdot 64$ & $11 \cdot 60$ & $15 \cdot 27$ & & Physostigmine. \\
\hline $65 \cdot 62$ & $3 \cdot 13$ & $31 \cdot 25$ & - & $\ldots$ & Purpurin. \\
\hline $65 \cdot 69$ & $5 \cdot 11$ & $29 \cdot 20$ & & $\cdots$ & Santalin. \\
\hline $65 \cdot 79$ & $5 \cdot 48$ & $25 \cdot 08$ & $3 \cdot 65$ & $\cdots$ & Rhœeadine. \\
\hline $\begin{array}{l}65 \cdot 85 \\
65.97\end{array}$ & $\begin{array}{l}5 \cdot 64 \\
5 \cdot 75\end{array}$ & $\begin{array}{l}28 \cdot 51 \\
20 \cdot 95\end{array}$ & $\dddot{7} \cdot 33$ & $\cdots$ & $\begin{array}{l}\text { Methysticin. } \\
\text { Chlorogenine. }\end{array}$ \\
\hline $\begin{array}{l}65 \cdot 97 \\
66.0\end{array}$ & $4 \cdot 6$ & 29.3 & ... & $\cdots$ & Mongumic acid. \\
\hline $66 \cdot 05$ & $8 \cdot 26$ & $25 \cdot 69$ & ... & $\cdots$ & Laserpitin. \\
\hline $66: 38$ & 6.38 & $27 \cdot 23$ & & 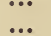 & Limonin. \\
\hline $66 \cdot 44$ & 6.57 & $22 \cdot 15$ & $4 \cdot 84$ & $\begin{array}{l}\cdots \\
\ldots\end{array}$ & Cocaïne. \\
\hline $66 \cdot 66$ & $9 \cdot 63$ & $23 \cdot 71$ & $\ldots$ & $\cdots$ & Hederic acid \\
\hline $66 \cdot 66$ & $3 \cdot 71$ & $29 \cdot 63$ & ... & $\ldots$ & Umbelliferon. \\
\hline $66 \cdot 67$ & 6.67 & $26 \cdot 66$ & $\ldots$ & $\ddot{\cdots}$ & Quassiin. \\
\hline $66 \cdot 67$ & $3 \cdot 70$ & $30 \cdot 63$ & $\ldots$ & $\ldots$ & Emodin. \\
\hline 66.98 & 6.98 & $26 \cdot 04$ & & $\cdots$ & Athamanthin. \\
\hline $67 \cdot 00$ & $9 \cdot 64$ & $16 \cdot 30$ & $7 \cdot 10$ & $\ldots$ & Gelsemine. \\
\hline $67 \cdot 11$ & $5 \cdot 43$ & $27 \cdot 46$ & & $\ldots$ & Brasillin. \\
\hline $67 \cdot 12$ & $11 \cdot 89$ & $11 \cdot 19$ & $9 \cdot 79$ & 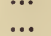 & Conhydrine. \\
\hline $67 \cdot 20$ & $7 \cdot 20$ & $25 \cdot 40$ & $\ldots$ & $\ldots$ & Rhinacanthin. \\
\hline $67 \cdot 41$ & $5 \cdot 62$ & $29 \cdot 96$ & $\ldots$ & $\ldots$ & Cubebin. \\
\hline $67 \cdot 41$ & $5 \cdot 62$ & $29 \cdot 96$ & 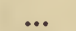 & $\ldots$ & Curcumin. \\
\hline $67 \cdot 5$ & $8 \cdot 4$ & $20 \cdot 5$ & $3 \cdot 6$ & $\cdots$ & Staphisagrine. \\
\hline $67 \cdot 6$ & $4 \cdot 2$ & $28 \cdot 2$ & $\ldots$ & $\ldots$ & Frangulic acid. \\
\hline $67 \cdot 71$ & $5 \cdot 88$ & $26 \cdot 41$ & $\ldots$ & $\cdots$ & Picropodophyllin. \\
\hline $67 \cdot 76$ & $6 \cdot 45$ & $25 \cdot 81$ & ... & $\cdots$ & Orcin. \\
\hline $67 \cdot 85$ & $3 \cdot 57$ & $28 \cdot 58$ & & $\cdots$ & Paracotoïn. \\
\hline $68 \cdot 06$ & $5 \cdot 08$ & $14 \cdot 32$ & $12 \cdot 34$ & $\cdots$ & Chelidonine. \\
\hline 68.07 & $5 \cdot 04$ & $26 \cdot 89$ & & & Erythrocentaurin. \\
\hline 68.08 & $6 \cdot 31$ & $17 \cdot 05$ & $8 \cdot 56$ & & Picroroccelline. \\
\hline $68 \cdot 22$ & $6 \cdot 66$ & $27 \cdot 65$ & $3 \cdot 45$ & $\begin{array}{l}\cdots \\
\ldots\end{array}$ & Moschatine. \\
\hline $68 \cdot 39$ & $7 \cdot 77$ & $16 \cdot 58$ & $7 \cdot 25$ & $\ldots$ & Ditaïne. \\
\hline $68 \cdot 85$ & $4 \cdot 92$ & $26 \cdot 23$ & $\ldots$ & $\ldots$ & Salicylous and benzoic acid. \\
\hline $68 \cdot 96$ & $8 \cdot 04$ & 23.00 & $\cdots$ & $\ddot{\ldots}$ & Elaterin. \\
\hline $69 \cdot 56$ & $7 \cdot 24$ & $23 \cdot 20$ & $\ldots$ & $\ldots$ & Betaorcin. \\
\hline $69 \cdot 72$ & $5 \cdot 42$ & $24 \cdot 86$ & $\ldots$ & $\cdots$ & Alkannin. \\
\hline $69 \cdot 76$ & $11 \cdot 62$ & $18 \cdot 61$ & ... & $\cdots$ & Capric acid. \\
\hline $69 \cdot 84$ & $4 \cdot 76$ & $25 \cdot 39$ & $\ldots$ & $\ldots$ & Cotoïn. \\
\hline 70.00 & $9 \cdot 29$ & $20 \cdot 71$ & 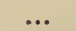 & $\ldots$ & Capsaïcin. \\
\hline 70.05 & $-6 \cdot 59$ & $16 \cdot 25$ & $7 \cdot 11$ & $\ddot{\cdots}$ & Cusconine and aricine. \\
\hline $70 \cdot 38$ & $8 \cdot 50$ & $21 \cdot 12$ & & $\cdots$ & Absinthiin. \\
\hline 70.58 & $7 \cdot 95$ & $16 \cdot 60$ & $4 \cdot 84$ & $\ldots$ & Atropine, hyoscyamine, etc. \\
\hline
\end{tabular}




\begin{tabular}{|c|c|c|c|c|c|}
\hline C. & H. & O. & N. & S. & NaME. \\
\hline $70 \cdot 58$ & $5 \cdot 88$ & 23.54 & & & Peucedanin. \\
\hline $70 \cdot 59$ & $5 \cdot 26$ & $19 \cdot 82$ & $4 \cdot 33$ & ... & Sanguinarine. \\
\hline 70.79 & $6 \cdot 20$ & $18 \cdot 88$ & $4 \cdot 13$ & $\cdots$ & Papaverine. \\
\hline 70.81 & $4 \cdot 35$ & $24 \cdot 84$ & & $\cdots$ & Chrysopicrin (vulpic acid). \\
\hline $70 \cdot 87$ & $7 \cdot 87$ & $15 \cdot 75$ & $5 \cdot 51$ & $\ldots$ & \\
\hline $\begin{array}{l}70 \cdot 87 \\
70 \cdot 9\end{array}$ & $\begin{array}{l}3 \cdot 94 \\
9 \cdot 5\end{array}$ & $\begin{array}{l}25 \cdot 19 \\
15 \cdot 6\end{array}$ & $\dddot{3} \cdot 9$ & $\begin{array}{l}\cdots \\
\cdots\end{array}$ & $\begin{array}{l}\text { Chrysophanic acid. } \\
\text { Delphinoïdine. }\end{array}$ \\
\hline $71 \cdot 00$ & 10.05 & $18 \cdot 95$ & ... & ... & Rottlerin. \\
\hline $71 \cdot 38$ & $5 \cdot 89$ & $22 \cdot 73$ & & ... & Pæoniofluorescin. \\
\hline $71 \cdot 58$ & $6 \cdot 67$ & $16 \cdot 84$ & $4 \cdot 91$ & ... & Morphine and piperine. \\
\hline $71 \cdot 64$ & $5 \cdot 08$ & $19 \cdot 10$ & $4 \cdot 18$ & $\ldots$ & Berberine. \\
\hline $72 \cdot 00$ & 8.00 & $10 \cdot 65$ & $9 \cdot 35$ & $\ldots$ & Menispermine (?) \\
\hline 72.09 & $12 \cdot 04$ & $15 \cdot 87$ & & $\ldots$ & Laurostearic acid. \\
\hline $72 \cdot 24$ & $7 \cdot 02$ & $16 \cdot 06$ & $4 \cdot 68$ & ... & Codeïne. \\
\hline $72 \cdot 31$ & $5 \cdot 22$ & $22 \cdot 47$ & & ... & Chrysarobin. \\
\hline $72 \cdot 41$ & $13 \cdot 79$ & $13 \cdot 79$ & ... & ... & Heptyl-alcohol. \\
\hline $72 \cdot 48$ & $11 \cdot 41$ & $16 \cdot 11$ & ... & ... & Ricinoleic acid. \\
\hline 72.58 & 8.06 & $19 \cdot 36$ & & ... & Pipitzahoic acid. \\
\hline $72 \cdot 90$ & $6 \cdot 54$ & $7 \cdot 48$ & 13.08 & ... & Harmaline. \\
\hline $72 \cdot 92$ & $5 \cdot 41$ & $21 \cdot 62$ & 0 & ... & Cinnamic acid. \\
\hline 73.03 & $7 \cdot 69$ & $10 \cdot 25$ & $8 \cdot 98$ & $\cdots$ & Quinamine. \\
\hline $73 \cdot 17$ & $7 \cdot 32$ & $19 \cdot 32$ & 1000 & $\cdots$ & Santonin, eugenol, eugenin. \\
\hline $73 \cdot 28$ & $3 \cdot 82$ & $12 \cdot 22$ & $10 \cdot 68$ & $\cdots$ & Indigo-blue. \\
\hline $73 \cdot 31$ & $6 \cdot 75$ & $15 \cdot 44$ & $4 \cdot 50$ & ... & Berberine and thebaine. \\
\hline 73.58 & $5 \cdot 67$ & $7 \cdot 54$ & $13 \cdot 21$ & $\cdots$ & Harmine. \\
\hline $73 \cdot 62$ & $11 \cdot 66$ & $14 \cdot 72$ & ... & ... & Bryoïdin. \\
\hline $73 \cdot 84$ & $13 \cdot 84$ & $12 \cdot 32$ & & ... & Caprylic alcohol. \\
\hline $73 \cdot 85$ & $8 \cdot 31$ & $4 \cdot 92$ & $12 \cdot 92$ & ... & Cytisine. \\
\hline $73 \cdot 68$ & $12 \cdot 28$ & $14 \cdot 04$ & $\ldots$ & ... & Myristic acid. \\
\hline $73 \cdot 97$ & $4 \cdot 11$ & $21 \cdot 92$ & & ... & Coumarin. \\
\hline 74.08 & $8 \cdot 62$ & ․ & $17 \cdot 28$ & $\ldots$ & Nicotine. \\
\hline $74 \cdot 4$ & $9 \cdot 7$ & $9 \cdot 57$ & $5 \cdot 62$ & $1 \cdot 37(\mathrm{P})$ & Chlorophyllan. \\
\hline $74 \cdot 54$ & 10.56 & $14 \cdot 90$ & 7.07 & $\cdots$ & Asclepin. \\
\hline $\begin{array}{l}74 \cdot 57 \\
74 \cdot 6\end{array}$ & $8 \cdot 48$ & $\begin{array}{r}9 \cdot 04 \\
21 \cdot 1\end{array}$ & $7 \cdot 81$ & $\cdots$ & $\begin{array}{l}\text { Aspidospermine. } \\
\text { Grönhartin. }\end{array}$ \\
\hline $\begin{array}{l}74 \cdot 6 \\
74 \cdot 66\end{array}$ & $7 \cdot 55$ & $17 \cdot 78$ & $\begin{array}{l}\cdots \\
\ldots\end{array}$ & $\begin{array}{l}\cdots \\
\ldots\end{array}$ & $\begin{array}{l}\text { Gronhartin. } \\
\text { Bixin. }\end{array}$ \\
\hline $75 \cdot 00$ & 3.57 & $21 \cdot 44$ & … & ... & Alizarin. \\
\hline $75 \cdot 00$ & $12 \cdot 50$ & $12 \cdot 50$ & $\ldots$ & $\ldots$ & Palmitic acid. \\
\hline $75 \cdot 02$ & $6 \cdot 66$ & $10 \cdot 43$ & $8 \cdot 64$ & $\ldots$ & Quinine and quinidine. \\
\hline 75 & $9 \cdot 07$ & $15 \cdot 89$ & & ... & Anacardic acid. \\
\hline $75 \cdot 59$ & $7 \cdot 09$ & $6 \cdot 29$ & $11 \cdot 02$ & ... & Paricine. \\
\hline $75 \cdot 78$ & $7 \cdot 37$ & $16 \cdot 85$ & ... & ... & Helleborin. \\
\hline 76.06 & $12 \cdot 68$ & $11 \cdot 26$ & $\ldots$ & $\ldots$ & Stearic acid. \\
\hline 76 & $12 \cdot 06$ & $11 \cdot 35$ & & $\cdots$ & Oleic acid. \\
\hline 76 & $12 \cdot 00$ & & $11 \cdot 20$ & $\cdots$ & Coniine. \\
\hline $76 \cdot 83$ & $8 \cdot 53$ & $14 \cdot 64$ & $\cdots$ & $\cdots$ & $\begin{array}{l}\text { Helenin. } \\
\text { Arachic acid. menthol }\end{array}$ \\
\hline 76.92 & $12 \cdot 82$ & $10 \cdot 26$ & & $\ldots$ & $\begin{array}{l}\text { Arachic acid, menthol, } \\
\text { capric aldehyde. }\end{array}$ \\
\hline $77 \cdot 07$ & $7 \cdot 95$ & $14 \cdot 98$ & & ... & Ostruthiin. \\
\hline $77 \cdot 24$ & $6 \cdot 54$ & $7 \cdot 30$ & $8 \cdot 9$ & $\ldots$ & Strychnine. \\
\hline $77 \cdot 55$ & $7 \cdot 48$ & $5 \cdot 44$ & 0.5 & $\ldots$ & $\begin{array}{l}\text { Cinchonine and cinchoni- } \\
\text { dine. }\end{array}$ \\
\hline $77 \cdot 69$ & $12 \cdot 23$ & & 10.07 & ... & Methylconiine. \\
\hline $77 \cdot 92$ & $11 \cdot 69$ & $10 \cdot 39$ & & $\ldots$ & Borneol. \\
\hline 78.05 & $10 \cdot 57$ & $\ldots$ & $11 \cdot 38$ & ... & Sparteïne. \\
\hline $78 \cdot 3$ & $11 \cdot 2$ & $?$ & 0 & $\ldots$ & $\begin{array}{l}\text { Conessine. } \\
\text { Aribine. }\end{array}$ \\
\hline $78 \cdot 43$ & $5 \cdot 68$ & $\cdots$ & 9 & & Aribine. \\
\hline
\end{tabular}




\begin{tabular}{|c|c|c|c|c|c|}
\hline C. & $\mathrm{H}$. & O. & N. & S. & NAME. \\
\hline $78 \cdot 57$ & $9 \cdot 52$ & $11 \cdot 91$ & $\ldots$ & $\cdots$ & Abietic acid. \\
\hline $78 \cdot 68$ & 13.95 & $7 \cdot 37$ & ... & $\ldots$ & Cetyl-alcohol. \\
\hline $78 \cdot 94$ & $10 \cdot 53$ & $10 \cdot 53$ & $\ldots$ & ... & Camphor and caryophyllin. \\
\hline $79 \cdot 02$ & $13 \cdot 17$ & $7 \cdot 81$ & ... & ... & Cerotic acid. \\
\hline $79 \cdot 24$ & $5 \cdot 65$ & $15 \cdot 11$ & ... & $\ldots$ & Benzaldehyde. \\
\hline $79 \cdot 47$ & $9 \cdot 93$ & $10 \cdot 59$ & & ... & Pimaric acid. \\
\hline $79 \cdot 74$ & $6 \cdot 33$ & $5 \cdot 06$ & $8 \cdot 86$ & $\cdots$ & Paytine. \\
\hline 80.00 & $9 \cdot 33$ & $10 \cdot 77$ & $\ldots$ & $\cdots$ & Thymol and carvol. \\
\hline $80 \cdot 25$ & $9 \cdot 55$ & $10 \cdot 20$ & $\ldots$ & ... & Cardol. \\
\hline $80 \cdot 67$ & $5 \cdot 88$ & $13 \cdot 45$ & $\ldots$ & ... & Cinnameïn. \\
\hline $81 \cdot 08$ & $8 \cdot 11$ & $10 \cdot 81$ & & $\cdots$ & Anethol. \\
\hline $81 \cdot 51$ & $13 \cdot 21$ & & $5 \cdot 28$ & $\ldots$ & Curarine. \\
\hline $81 \cdot 81$ & $14 \cdot 14$ & $4 \cdot 05$ & $\ldots$ & ... & Cerotyl-alcohol. \\
\hline $81 \cdot 82$ & 6.06 & $12 \cdot 12$ & $\ldots$ & $\cdots$ & $\begin{array}{l}\text { Styracin and cinnamic } \\
\text { aldehyde. }\end{array}$ \\
\hline $81 \cdot 82$ & $11 \cdot 04$ & $7 \cdot 14$ & $\ldots$ & ... & Euphorbone and lactucerin. \\
\hline $82 \cdot 19$ & $14 \cdot 15$ & $3 \cdot 66$ & ... & $\ldots$ & Melissyl alcohol. \\
\hline $82 \cdot 44$ & $11 \cdot 41$ & $6 \cdot 15$ & $\ldots$ & $\ldots$ & Hydrocarotin. \\
\hline $82 \cdot 57$ & $11 \cdot 36$ & 6.06 & $\ldots$ & $\ldots$ & Betulin. \\
\hline $83 \cdot 49$ & $11 \cdot 79$ & $4 \cdot 73$ & ... & ... & Amyrin. \\
\hline $83 \cdot 87$ & $11 \cdot 83$ & $4 \cdot 30$ & $\ldots$ & $\cdots$ & Phytosterin. \\
\hline $84 \cdot 11$ & $12 \cdot 15$ & $3 \cdot 74$ & ... & $\ldots$ & Cholesterin. \\
\hline $84 \cdot 37$ & $9 \cdot 37$ & $6 \cdot 26$ & ... & $\ldots$ & Carotin. \\
\hline $88 \cdot 24$ & $11 \cdot 76$ & $\ldots$ & $\ldots$ & $\cdots$ & Caoutchouc, terpenes, etc. \\
\hline $92 \cdot 31$ & $7 \cdot 69$ & & $\ldots$ & $\ldots$ & Styrol. \\
\hline
\end{tabular}




\section{N D E X .}

\section{Abietite, 225}

Absinthiin, 49, 146

Acacia, tannin of, 162

Acid, abietic, 127

acetic, $23,24,119,226$

aconitic, 70

acrylic, 24, 119

anacardic, 146

angelic, 13, 24, 119

anthemic, 146

arabic, $76,210,211,250$

arachic, 15

aspartic, 206

atranoric, 151

beberic, 146

benzoic, 32, 33, 35, 49, 226

beta-erythric, 151

boheic, 160

butyric, 35, 119

caffeic, 161

caffeo-tannic, 161

cambogic, 135

capric, 13,119

caproic, 13, 119

caprylic, 13, 119

carbusnic, 150

catechuic, 41, 44, 156

, estimation, 157

catechu-tannic, 156

cathartic, 86,248

cathartogenic, 248

celastrus-tannic, 163

cetraric, 151

chelidonic, 148

chrysophanic, 36, 132

cinchona-nova-tannic, 163

cinchona-tannic, 162

cincho-tannic, 162

cinnamic, 25

citric, 70

" estimation of, 226,228

" reactions of, 226

crotonic, 119

diorsellic, 149

ellagic, 153
Acid, ellago-tannic, 160

erythric, 151

evernic, 150

everninic, 150

formic, $23,24,119,226$

filix-tannic, 162

frangulic, 133

fumaric, 70,232

gallic, $32,133,226$

" detection and estimation, 47,137

gallo-tannic, 160, 226

gelsemic, 205

estimation, 159

glutamic, 207

glycolic, 233

glycyrrhizic, 171

gummic, 210

gyrophoric, 150

helianthic, 170

hydrocarbusnic, 151

hydrocyanic, 24, 29

isobutyric, 119

jalapic, 140

jervic, 148

kinic, 232

lactic, 232

lauric, 13, 112

lecanoric, 149, 151

leditannic, 163

lichenostearic, 151

linoleic, 11

lobaric, 151

estimation, 111

maleic, 232

malic, 70, 229, 234

, detection, 225

meconic, 148

melangallic, 137

melilotic, 108

metapectic, 211

metarabic, 88, 209, 211, 235, 243

metatungstic, 56

methylcrotonic, 13, 119

Đethylsalicylic, 30 
Acid, mongumic, 127

morintannic, 158

myristic, 15, 16, 112

myronic, 165

nitric, 78

,, estimation, $83,84,85$

nucitannic, 163

octylic, see caprylic

œnanthic, 119

oleic, 11, 112, 113

" detection, 18

" estimation, 111

ophelic, 147

orsellic, 149

oxalic, $70,91,230$

oxyusnetinic, 150

palmitic, 18, 112

para-oxybenzoic, 35,36

parellic, 150

patellaric, 150

pectic, 211

pelargonic, 13

phosphomolybdic, 56

phosphoric, 226, 229

phyllic, 127

picric, 56

pimaric, 127

pinic, 127

pipitzahoic, 136

podocarpic, 127

podophyllic, 139

polygonic, 149

polyporic, 90

propionic, 119

protocatechuic, 35

pteritannic, 163

quercitannic, 161

quinic, see kinic

quinovic, 175

racemic, 70, 229

rhatania-tannic, 157

ricinoleic, 19

rocellic, 149

ruberythric, 134

rubichloric, 232

rufigallic, 137

salicylic, $24,32,33,49$

salicylous, 24, 29, 168

santonic, 36 (see also 'santonin ')

sclerotic, 86,248

stearic, 18, 112

stictic, 151

succinic, 230, 231

sulphuric, 226

sylvic, 127

taigusic, 136

tannaspidic, 162

tannic, 56

tartaric, 71

$" \quad$ estimation, 228
Acid, toxicodendric, 24

trimethylacetic, 119

usnic, 150, 152

valerianic, $13,24,35,119$

viridic, 161

vulpic, 150

Acids, 225

amidic, 247

estimation of in fruits, 71

examination for, 65,69

examination of substances soluble in, 91

extraction with, 91

fatty, identification of, 120

," separation from resin, 112

lichen, 149

mineral, estimation of, 71

" tests for, 71

organic, estimation of, 69

produced by alkalies, 36

qualitative separation of, 70

resin, 32,127

tannic, estimation of, 41-47

volatile, $23,29,117$

Acolyctine, 58

" separation of, 119

Aconine, 58

Aconitine, 50, 179, 181

estimation of, 60

Acrinyl, sulphocyanate of, 166

Adansonin, 146

Aesculetin, 169

Aesculin, 49, 169

Agrostemma githago, saponin in, 68, 69

Albumen, estimation of, $79,80,238$ soluble in dilute soda, 88 vegetable, in mucilage, 66

Albumenoids, detection of, 78 estimation of, 234, 236, 237

examination for, 65,78

extracted by dilute acid, 240

extracted by pepsin, 240

extracted by spirit, 241

extraction of, 78

insoluble in dilute soda, 89

microchemical, detection of, 78

nitrogen in, 234

not precipitated by alcohol, 76

reagents for, 79

soluble in dilute soda, 88,235

Alchornin, 146

Alcohol, examination of substances soluble in, 38

extraction with, 38

amyl, 30

cerotyl, 13, 110

cetyl, 13,110

melissyl, 13,110

melyl, see melissyl 
Alcohol, octyl, 30

Alcohols, boiling points of, 30

Alcohols, primary, secondary, etc., 30

Aldehyde, angelic, 29

$$
\text { benzoic, } 25
$$

capric, 29

cinnamic, 25,29

methyl-capric, 29

pelargonic, 29

salicylic, 25,29

Aldehydes, detection in ethereal oils, 29

Alder, tannin of, 156

Aleurites laccifera, wax from, 110

Aleurone, 236

Algarobilla, tannin of, 159

Alizarin, 133

Alkaloid, amorphous, separation from

cinchona, 194

of celandine, 50

of eschscholtzia, 204

of pimento, 50

Alkaloids, 178

colour-reactions of, $178,179,180$

confirmatory tests for, 57

decomposition by alkalies, 58

estimation of, $58,63,182$

examination for, 50,51

extracted in fixed oil, 19

extracted with alcohol, 38,48

extracted with ether, 33

extracted with petroleum spirit, 20

extraction from aqueous solution, 49

group-reagents for, 55

isolation of, 55

microsublimation of, 181

not separated by shaking, 57

of cinchona, 198

$$
\text { " rarer, } 198
$$

" separation, 194

platinum and gold salts of, 181

quantitative separation, 194

separation, $63,189^{\circ}$

separation by precipitation, 193

separation by solvents, 191

tests for, 181

volatile, 50

Alkannin, 135

Almond, oil of sweet, 102

Aloe-resin, 177

Aloes, valuation of, 177

Aloins, various, 176

Alstonine, 203

Amanitine, 205

Amides, 205

Amido-compounds, 82

Amidulin, 249

Amines, 244

$$
\text { estimation of, } 245
$$

Ammonia, estimation of, 81
Ammonia, examination for, 78

Amygdalin, 164

Amylin, 253

Amylodextrin, 250

Amylum, see starch.

Amyrin, 109

Analysis, general method, 5

Anemonin, 109

Anemonol, 109

Angelicin, 109, 208

Anhydrides, action of alkalies on, 36

Aniline, 50

Anthochlor, 117

Anthoxanthin, 117

Anthracene, 136

Anthraquinone-derivatives, 127,131 , 136

Antiarin, 175

Antirin, 146

Aphrodæscin, 170

Apiin, 170

Apricots, oil of, 102

Arabin, 210

Arabinose, 218

Arbutin, 167

Argyræscin, 170

Aribine, 203

Aricine, 198

Aristolochia, bitter, 175 yellow, 146

Arnicin, 146

Asaron, 108

Asclepiadin, 146

Ash, estimation of, 7

Asparagine, 82, 206, 207

Aspidospermine, 50, 204

Athamanthin, 145

Atherospermine, 203

Atropine, 50 estimation of, 60,182

Bablah fruits, tannin of, 160

Beberine, 203

Beech-oil, 102

Belladonnine, 203

Benzohelicin, 169

Berberine, 49 estimation of, 62

Betaine, 205

Betaorcin, 152

Betapicroerythrin, orsellinate of, 151

Betulin, 145

Birch, tannin of, 162

Bistort, tannin of, 158

Bitter-almond oil, 29

Bitter principles, 127 extracted by alcohol, 38,48

lead compounds of, 52

Bixin, 135

Brasillin, 137 
Brucine, 50 estimation of, 61,183

Bryoidin, 109

Bryonin, 170

Butylalanine, 205

Cacao, 187

Caffeine, 49 estimation of, 186

Cailcedrin, 146

Calabar Bean, estimation of alkaloid, 184

Calabarine, estimation of, 184

Calcium, oxalate of, 91 estimation, 91 microscopical detection, 92

Calendulin, 175

Californin, 175

Calycin, 151

Cane-sugar, 220

Caoutchouc, detection in fixed oil, 11 extraction of, 109

Capsaicin, 109

Capsicin, 109

Capsicum, 49 alkaloid of, 50

Caragheen-sugar, 219

Carapin, 175

Carbohydrates, see under respective names.

Carotin, 109

Cardol, 146

Caryophyllin, 49, 146

Carthamin, 178

Cascarillin, 49, 146

Casein, 235

Castor-oil, 102

Catechin, 32, 138 estimation of, 137

Catechu, tannin of, 156

Celandine, alkaloid of, 50

Celastrus, tannin of, 163

Cell-nucleus, 79

Cellulose, 252, 256 estimation of, 96 varieties of, 256

Ceratophyllin, 150

Cerosin, 111

Cerotene, 110

Cevadilla seed, 184

Chamælirin, 172

Chelidonine, estimation of, 62

Chelidonium, estimation of alkaloid in, 184

Chenopodine, 208

Chimaphilin, 147

Chiratin, 147

Chlorogenine, 203

Chlorophyll, 19, 113, 114 estimation of, 115
Chlorophyll, extraction of, 19, 32

Chlorophyllan, 114, 115

Cholesterin, 99 detection in fixed oil, 11 detection and estimation, 106

Choline, 205

Chrysarobin, 132

Chrysin, 128

Chrysophyll, 114

Chrysopicrin, 150

Chrysorhamnin, 135

Chylariose, 218

Cicutin, 147

Cinchona, amorphous alkaloid of, 191 tannin of, 162

Cinchona-alkaloids, estimation of, 62

Cinchona-nova-red, 163

Cinchona-red, 162

Cinchonidine, separation of, 194

Cinchonine, 49, 50 separation of, 194

Cinnamein, 25

Cinnamyl, cinnamate of, 25

Cnicin, 176

Cnicus benedictus, bitter principle of, 49

Cocaine, 203

Codeine, 50

Colchiceine, 49

Colchicine, 49 estimation of, 61

Colocynthin, 49, 170

Columbin, 147

Concluding remarks (to Part I.), 97

Conessine, 203

Conglutin, 235

Conhydrine, 50

Coniferin, 167

Coniine, 50 estimation of, $61,183,184,189$

Conquinine, see quinidine

Convallamarin, 49, 172

Convallarin, 172

Convolvulin, 141

Coriamyrtin, 143, 170

Corydaline, 203

Cotoin, 147

Cotton-seed, oil of, 102

Coumarin, 108

Cratægin, 175

Crocetin, 171

Crocin, 171

Crude Fibre, 257

Crystalloids, 79

Cubebin, 49, 145

Curarine, 58, 179, 194, 202

Curcumin, 135

Cusconine, 198

Cusparin, 175

Cuticular substance, 95, 253

Cutose, 253 
Cyanophyll, 113

Cyclamin, 172

Cyclopiafluorescin, 163

Cyclopia-red, 163

Cyclopin, 163

Cynanchocerin, 108

Cytisine, 203

Daphnin, 49, 168

Datiscin, 170

Delphinine, 50

Delphinoidine, 50

Dextrin, 212

alcoholate of, 213

estimation of, $65,67,213$

estimation of in cane-sugar, 215

Dextrose, see glucose

Diastase, 237

Diethylamine, 244

Digitalein, 49, 143, 173

Digitalin, 142, 173

Digitaliresin, 142

Digitin, 143

Digitonein, 143

Digitonin, 143, 173 estimation of, 69

Digitoresin, 143, 174

Digitoxin, 142

Dimethyloreoselon, 145

Diosmin, 108

Distillation, fractional, 124

Ditaïne, 204

Ditamine, 204

Divaleryloreoselon, 145

Divi-divi, tannin of, 156,160

Dulcamarin, 171, 204

Dulcite, 225

Ecboline, 202

Echicerin, 108

Echitamine, 204

Elaidin, test for oils, 102

Elaterin, 49, 147

Emetine, 50 estimation of, 61

Emodin, 132

Emulsin, 237

Ergotine, 202

Ergotinine, 202

Ericinol, 143

Ericolin, 49, 143, 166

Erythrite, diorsellinate of, 151 orsellinate of, 151

Erythrocentaurin, 147

Erythrophyll, 115

Erythrophlœine, 202

Erythroretin, 132

Erythrosclerotin, 134

Eschscholtzia, 204

Ethereal Oil, see 'Oil, ethereal'
Ethereal Salts, see 'Salts, ethereal'

Ether, direct extraction with, 36

estimation of substances soluble in, 32

examination of substances soluble in, 31

Etiolin, 116

Ethylamine, 244

Eucalyn, 219

Eupatorin, 147

Euphorbon, 108

Fat-acids, fixed, 14

fractional precipitation of, 14

free, 105

detection and estimation, 106

melting points of, 14,15

volatile, 13

Fats, see 'Fixed Oil'

Fehling's Solution, 72

Ferments, 237

Fibrin, vegetable, 235

Filix, tannin of, 162

Filicin, 99, 107

Fixed oil, composition of, 10

detection of, 10

elaidin test for, 10

estimation of, 10, 11, 99

estimation of glycerin in, 12

linoleic acid in, 11

oleic acid in, 11

qualitative reactions, 11

resinification of, 101

tests for, 101, 102

Fraxin, 169

Fresh plants, treatment of, 6,10

Fruit-sugar, 218

Fumarine, 205

Fungin, 253

Galactose, 219

Galls, tannin of, 156, 159, 226

Gardenin, 127

Greissospermin, 49, 204

Gelose, 252

Gelsemine, 50, 205

General Remarks, 1

Gentian-bitter, 140

Gentisin, 139

Geraniin, 176

Glaucine, 205

Gliadin, 241 properties of, 242

Globularin, 170

Globulin, 235. estimation of, 79

Glucodrupose, 256

Glucolignose, 256

Glucose, detection of, 214 estimation of, $72,73,74,215,217$

$18-2$ 
Glucose, fermentation test for, 216 polarization of, 221

Glucoses, detection and estimation, 64,72 extracted by alcohol, 38,48 various, 256

Glucosides, detection of, 53 direct examination for, 50 extraction of by ether, 33 extraction of from aqueous solution, 49 group-reagents for, 54 solubility of, 164

Glutamine, 82 detection of, 207 estimation of, 207

Gluten, 243

Glutencasein, 235

Gluten fibrin, 241, 242

Glutin, 241

Glycerides, 11

Glycerin, estimation of, 109

Glycyrrhizin, 171

Goa-powder, 132

Gold, chloride of, as alkaloid reagent, 56

Granulose, 249

Grape-sugar, see glucose

Gratiolin, 49, 172

Grönhartin, 136

Ground nut, oil of, 102

Guacin, 147

Guarana, 186

Guaranine, 186

Gum, 208

Gum arabic, varieties of, 211

Gum, see also 'Mucilage'

Gummicose, 210

Gum-resins, commercial, 129

Gypsophila struthium, saponin in, 69

Hæmatoxylin, 32, 33, 136

Harmaline, 203

Harmine, 203

Hazel nut, oil of, 102

Helenin, 108

Helianthus, oil of, 102

Helleborein, 49, 172

Helleborin, 172

Hemp, oil of, 102

Hesperidin, 171

Hesperidin-sugar, 225

Homofluorescin, 151

Hop-bitter, 147

Hop-resin, 49

Hop, tannin of, 156

Horse chestnut, tannin of, 158

Humus, 90

Hurin, 148

Hydrastine, 205

Hydrocellulose, 250
Hydrocarotin, 109

Hydrocotoin, 147

Hygrine, 203

Hyoscine, 60

Hyoscyamine, 50

estimation of, 60,183

Hypochlorin, 114, 116

Incrusting substance, 95,253

Indican, 174

Indigo blue, 174

Indiglucin, 174

Indigo white, 174

Inosite, 219

Introduction, 1

Inulin, characters, 87 detection of, 66 estimation and extraction of, 86 examination for, 86

Inuloid, 87

Invertin, 237

Invert sugar, 218

Ipecacuanha, tannin of, 163

Isodulcite, 225

Isophlorrhizin, 169

Jalapin, 140

Jalapinol, 140

Jervine, 180

Juniperin, 148

Jurubebine, 205

Kämpferid, 108

Kawain, 148

Knoppern-galls, tannin of, 159

Kosin, 107

Lactose, 210

Lactucerin, 108

Lactucin, 176

Lactucon, 108

Laserol, 145

Laserpitin, 145

Laurocerasin, 164

Legumin, estimation of, 79, 234

Leucine, 207 estimation of, 207

Leucotin, 147 .

Levulin, 212 alcoholate, 213

detection and estimation of, 67, 213

Levulosan, 220

Levulose, 218

Lichen-acids, test for, 151

Lichenin, 249, 251

Lichens, microscopical examination of, 151

Lichen-starch, 251

Lignin, 95, 252, 253 
Lignin, micro-chemical characters of, 255

Lignin, micro-chemical detection of, 95

Ligustrin, 170

Limonin, 171

Linin, 176

Linseed, oil of, 102

Liriodendrin, 148

Literature of plant-analysis, 3

Lobeliine, 50, 202

Loturine, 175, 205

Lupinin, 176

Lutein, 117

Luteolin, 140, 178

Lycine, 205

Lycopin, 148

Lycopodine, 205

Maclurin, 158

Maltose, 221

Mangostin, 148

Mannite, 77, 224

Marattin, 70

Marrubin, 148

Masopin, 148

Meconin, 148

Melampyrite, 225

Melanthin, 173

Melanthigenin, 174

Melezitose, 221

Melitose, 221

Melting-points, determination of, 15

Menispermine, 205

Menyanthin, 49, 166

Mercuric chloride as alkaloid reagent, 56

Metacellulose, 253

Methylanthracene, 136

Methylconiine, 50

Methysticin, 148

Milk-sugar, 220

Moisture, estimation of, 5

Monamines, 244

Morin, 158

Morphine, 50 estimation of, $61,184,199$

Morindin, 135

Morindon, 135

Mucedin, 241, 242

Mucilage, characters of, 210 estimation of, 65 examination for, 65 modified method of examination, 209 vegetable, 208

Mudarin, 176

Munjestin, 135

Murrayin, 171

Muscarine, 205
Mustard, volatile oil of, 166

Mycose, 221

Myosin, 235

separation from vitellin, 236

Myrica cerifera, wax from, 110

quercifolia, " " 110

species, ,", 110

Myrobalans, tannin of, "156, 160

Myrosin, 165

Myroxocarpin, 108

Narceine, 49,50

Narcotine, 50 estimation of, $61,184,199$

Naringin, 171

Narthecin, 148

Nepaline, 60

Neurin, 205

Nicotine, 50 estimation of, 61,188

Nitriles, 27

Nitrogen, detection in ethereal oil, 27 estimation of, 80

Nitrogenous substances, 244

Nucin, 148

Nucite, 219

Nupharine, 50, 205

Oil, ethereal, constituents of, 27

detection and estimation of, 21

detection of nitrogen in, 27

detection of sulphocyanogen in, 27

detection of sulphur in, 26

distillation of, 23

estimation of, 117

examination of, 25

examination for aldehydes, 29

fluorescence, 26

fractional distillation, 27 , 124

optical tests for, 120

polarization of, 25

reactions of, 26, 121, 122, 123

solubility in alcohol, 26, 120

specific gravity of, 26

olive, 102

stearoptenes in, 28

Oleandrine, 205

Olivil, 176

Ononin, 170

Operations, preliminary, 5

Opium, estimation of alkaloid in, 199

Orcin, estimation of, 152

Oreoselon, 145

Ostruthiin, 144

Oxyacanthine, 205 
Oxyaloin, 178

Oxycyclopin, 163

Oxyleucotin, 147

Oxyneurin, 205

Pæoniofluorescin, 36, 131

Panaquillon, 172

Papaverine, 49, 50

Papayotin, 237

Paracellulose, 253

Paracholesterin, 107

Paracotoin, 147

Paramenispermine, 205

Pararabin, 91 estimation of, 93

- Paricine, 199

Paridin, 172

Parigenin, 174

Parillin, 174

Paytine, 199

Pectin, 208

Pectose, 253

Pelletierine, 205

Peptone, 239

Pereirine, 50, 205

Petroleum Spirit, estimation of substances soluble in, 8

Petroleum Spirit, extraction with, 8

Peucedanin, 145

Phæoretin, 132

Phaseomannite, 219

Philygenin, 169

Philyrin, 169

Phlobaphene, 88, 90

Phloroglucin, 35

Phlorose, 218

Phlorretin, 169

Phlorrhizin, 169

Phyllocyanin, 113

Phylloxanthin, 114, 116

Physalin, 49, 171

Physostigmine, 50 estimation of, 61,184

Phytosterin, 107

Picroerythrin, 151

Picrolichenin, 151

Picropodophyllin, 139

Picrosclerotine, 202

Picrotoxin, 49, 142

Pilocarpine, 50 estimation of, 184

Pimento, alkaloid in, 50

Pine, tannin of, 162

Pinipicrin, 167

Pinite, 225

Piperine, 49 estimation of, 188

Pittosporin, 170

Platinum, perchloride of, as alkaloid reagent, 56
Plumbagin, 149

Podophyllotoxin, 139

Podophyllum peltatum, 139

Polychroite, 171

Pomegranate, tannin of, 160

Poppyseed, oil of, 102

Populin, 49, 168

Porphyrine, 203

Potassiobismuthic iodide, as alkaloid reagent, 55

Potassiocadmic iodide, as alkaloid reagent, 56

Potassiomercuric iodide, as alkaloid reagent 55

Potassium, bichromate of, as alkaloid reagent, 56

Potassium, myronate, 165 tribromide, as alkaloid reagent, 55

Potassium, tri-iodide, as alkaloid reagent, 55

Powdering, 6

Preliminary operations, 5

Protoplasm, 79

Pseudaconitine, 60

Pseudamyl-alcohol, 30

Punicine, 205

Purpurin, 133

Pyrocatechin, 32, 33, 36, 138

Pyrogallol, 35, 36, 137

Quassiin, 49, 149

Quebrachin, 49

Quercetin, 36, 138

Quercin, 176

Quercite, 225

Quercitrin, 36, 138, 178

Quillaja saponaria, saponin in, 69

Quinamine, 198

Quinidine, 50 separation of, 194

Quinine, estimation of, 62,185 separation from bark-alkaloids, 194

Quinovin, 175

Rape-oil, 102

Ratanhin, 208

Resin, detection in fixed oil, 11 extraction of, 31,38

micro-chemical detection, 33

separation from fat acid, 112

Resins, 127 acid, 34, 36

, separation of, 127,128

action of potash on, 34

anhydrides, 34

behaviour to reagents, 34

commercial, 129

dry distillation of, 36

indifferent, 34 
Resins, micro-chemical examination, 33 oxidation-products of, 34 purification, 34

Resorcin, 35

Rhamnin, 135

Rhamnodulcite, 225

Rhatany-red, 157

Rhatany-root, tannin of, 157

Rhinacanthin, 135

Rhinacanthus communis, 135

Rhinanthin, 163

Rhinanthogenin, 163

Rhœeadine, 203

Rhoeaginine, 203

Rhubarb, Assay by Iodine, 248

Rhus succedanea, wax from, 110

Ricinus communis, oil of, 102

Robinin, 140, 178

Rottlerin, 149

Rubiadin, 134

Rubian, 134

Rubiretin, 134

Rutin, 140, 178

Sabadilline, 50 estimation of, 61,184

Sabatrine, 50 estimation of, 61,184

Saccharose, Böttger's test for, 76 characters of, 76 estimation of, 75 examination for, 65,72 inversion of, 75

Saccharoses, 220

Salicin, 33, 50, 168

Salicin-sugar, 218

Saligenin, 168

Saliretin, 168

Salts, ethereal, 29

Samaderin, 170

Sand, 7

Sanguinarine, 62

Santalin, 137

Santonin, 36,49 estimation of, 141

Saponaria officinalis, saponin in, 69

Sapogenin, 68

Saponin, 49, 173 estimation of, 68 examination for, 67

Sarracenia purpurea, alkaloid in, 50

Sarsaparilla, saponin in, 69

Scillain, 173

Sclererythrin, 134

Scleromucin, 249

Scleroxanthin, 149

Senegin, 49, 173

Separation, methods of, 3

Sesamé, oil of, 102

Sicopirin, 149
Sinalbin, 166

Sinapine, acid sulphate of, 166 sulphocyanate of, 166

Sinistrin, 212 alcoholate of, 213 detection and estimation, 67, 213

Sinkaline, 205

Smilacin, 174

Soda, examination of substances soluble in, 88

Solanidine, 49

Solanine, 50

Sorbin, 219

Sorbite, 225

Sordidin, 151

Sparattospermin, 176

Sparteine, 50, 205

Special Methods, 99

Staphysagrine, 180, 193

Starch, 91, 249 estimation of, 93

Stearoptene in ethereal oil, 28

Styracin, 25

Strychnine, 50 estimation of, 61,183

Styrol, 108

Suberin, 95, 255

Sugar, estimation by polarization, 221

Sulphocyanogen, detection in ethereal oil, 27

Sulphur, detection in ethereal oil, 26

Sumach, tannin of, 159

Sunflower seed, oil of, 102

Surinamine, 203

Syringin, 49, 170

Syringopicrin, 170

Tampicin, 140

Tanacetin, 149

Tanghinin, 149

Tannin, constitution of, 152 decomposition of, $152,153,154$, 156

detection of, 39

detection of glucose from, 153

ellagic acid from, 153

estimation of, 41.47

extracted by ether, 31

$$
\text { ," alcohol, } 38
$$

extraction of, 40

gallic acid from, 153

glucosidal nature of, 153

insoluble in water, 156

microscopical detection of, 40

purification of, 155

reactions of, 40

separation from alkaloid, etc., 52

various, 153, 162, 163

Taraxacin, 149

Taxine, 50, 205 
Tea, 186

tannin of, 160

Thebaine, 50

Tectochrysin, 128

Terpenes, 28

Thalictrin, 180

Theine, estimation of, 62

Theobromine, 49 estimation of, 187

Thevetin, 172

Thujin, 140

Toxiresin, 143

Trehalose, 221

Triethylamine, 244

Trimethylamine, 245

Trimethylaniline, 50

Triticin, 212

alcoholate of, 213

detection and estimation, 67, 213

Turpethin, 141

Tyrosine, detection of, 208 estimation of, 207

Umbelliferon, 36

Valonia, tannin of, 159

Vanillin, 167 estimation of, 144

Variolinin, 151

Vasculose, 253

Verantin, 134

\begin{tabular}{|l} 
Veratrine, 50 \\
$\quad$ estimation of, 61,184 \\
Veratroidine, 179, 189 \\
Viola tricolor, 139 \\
Violaquercitrin, 139 \\
Violin, 203 \\
Vitellin, 235 \\
Vitellin, isolation of, 236
\end{tabular} separation from myosin, 286

Walnut, oil of, 102

Water, examination and estimation of substances soluble in, 65 mineral matter dissolved by, 65

Wax, Bahia, 111 Carnauba, 111 microscopical detection, 111 vegetable, 13,110

Wood-gum, 249, 252

Wormseed, estimation of santonin in, 141

Wrightine, 203

Xanthein, 117

Xanthin, 117

Xanthophyll, 114, 116

Xanthorhamnin, 135

Xanthosclerotin, 149

Xylostein, 149

Zeorin, 151.

THE END. 



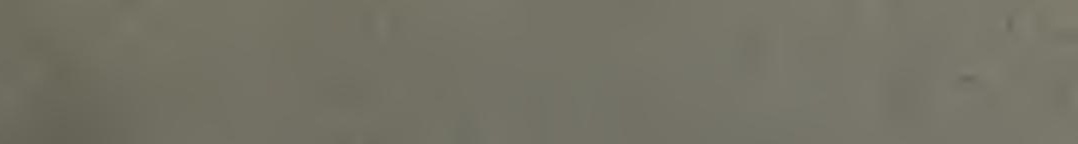

\section{$\times$}

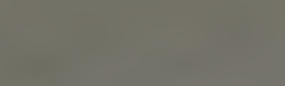

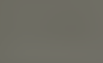

(1) 11

$\frac{15}{25}$

3

i)

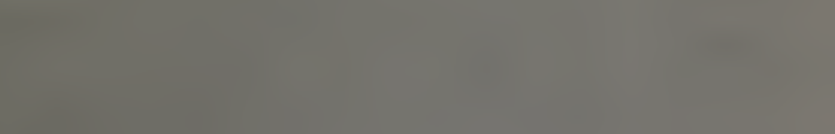

$-$

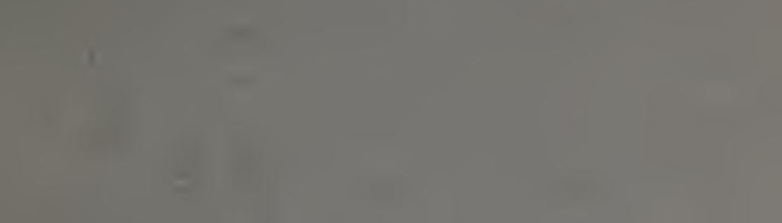

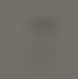

(

$=1$

(1) 



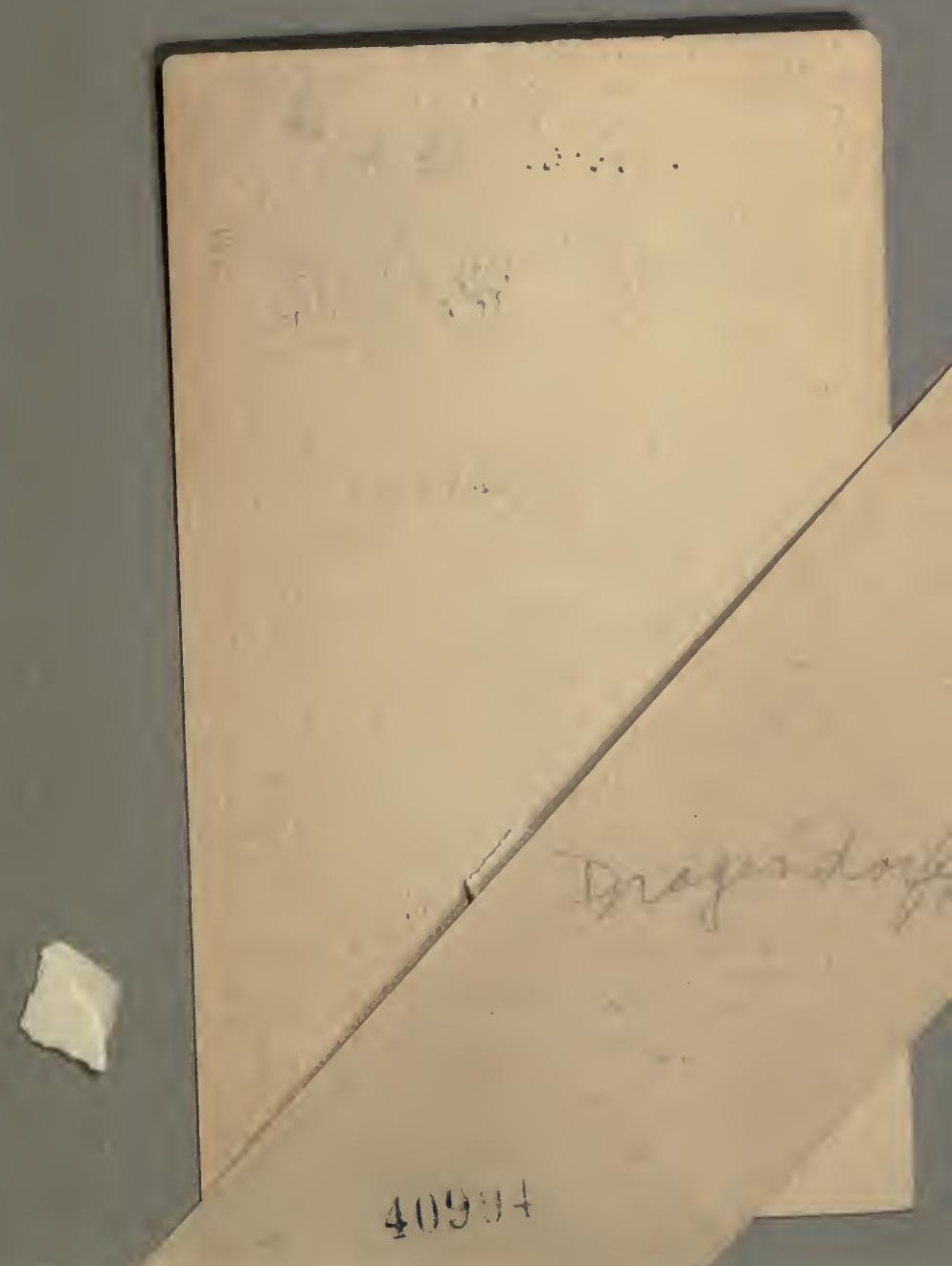


